\title{
The Role of Hydrogen Abstraction Reaction in Photocatalytic Decomposition of High Energy Density Materials
}

\author{
Roman Tsyshevsky ${ }^{1}$, Anton S. Zverev ${ }^{2}$, Anatoly Y. Mitrofanov², Natalya N. Ilyakova ${ }^{2}$, \\ Mikhail V. Kostyanko ${ }^{1,3}$, Sergey V. Luzgarev $^{2}$, Guzel G. Garifzianova ${ }^{4}$, and Maija M. \\ Kuklja ${ }^{1, *}$ \\ ${ }^{1}$ Department of Materials Science and Engineering, University of Maryland, College Park, MD 20742, USA \\ ${ }^{2}$ Department of Organic and Physical Chemistry, Kemerovo State University, Kemerovo, Russia \\ ${ }^{3}$ Federal Research Center of Coal and Coal Chemistry, Siberian Branch of the Russian Academy of \\ Sciences, Kemerovo, Russia \\ ${ }^{4}$ Department of Catalysis, Kazan National Research Technological University, Kazan, Russia
}

\section{Supplementary Information}

\section{Surface slab models and details of calculations}

Solid state periodic calculations were performed by density functional theory (DFT) with optPBE-vdW ${ }^{1,2,3,4,5}$ functional, which includes corrections for van der Waals interactions as implemented in the VASP code ${ }^{6,7,8}$. The projector augmented-wave (PAW) pseudo-potentials ${ }^{9}$ were used. Minimal energy paths in the VASP periodic calculations were obtained with the nudged elastic band method ${ }^{10}$ with six intermediate images. Atomic positions were relaxed using conjugate gradient and quasi-Newtonian methods within a force tolerance of $0.05 \AA / \mathrm{eV}$.

In simulating an ideal PETN crystal (Figure S1a), we used $2 \times 2 \times 2$ Monkhorst-Pack k-point mesh with a kinetic energy cut-off of $520 \mathrm{eV}$, whereas hybrid functional calculations were performed with a k-point at the $\Gamma$-point only. Atomic coordinates and lattice constants were allowed to simultaneously relax without any symmetry constraints. The convergence criterion for electronic steps was set to $10^{-5} \mathrm{eV}$, and the maximum force acting on any atom was set not to exceed 0.02 eV/A. The calculated lattice constants of the tetragonal PETN unit cell using optPBE-vdW functional, $\boldsymbol{a}=\boldsymbol{b}=9.331 \AA, \boldsymbol{c}=6.624 \AA$ agree with the experimental lattice vectors of $\boldsymbol{a}=\boldsymbol{b}=9.38 \AA, \boldsymbol{c}=$ $6.71^{11}$ within $\sim 1 \%$. The lattice constants calculated using PBE $^{12}$ functional $\boldsymbol{a}=\boldsymbol{b}=9.64 \AA, \boldsymbol{c}=6.82 \AA$ are also in reasonable agreement with experimental from lattice constants (average deviation is $2.5 \%)$.

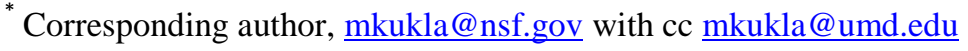


To simulate light absorbing molecule on the PETN (110) surface using optPBE-vdW functional, a slab model was used with the supercell lattice vectors of $a=14.77 \AA, b=14.77 \AA$, and $c=45.79 \AA$ and $\alpha=\beta 90^{\circ}, \gamma=53.3^{\circ}$ (Figure S1b). As optPBE-vdW failed to predict reaction path for hydrogen atom transfer reaction proceeding on the PETN (110) surface, PBE functional and the surface slab model with the supercell parameters $a=13.64 \AA, b=13.63 \AA$, and $c=46.41 \AA$ and $\alpha=\beta=\gamma=90^{\circ}$ (Figure S1c). were employed. For the calculations at PBE level of theory, the surface was cut from the PETN crystal which lattice and ionic parameters were relaxed using the same functional. In both surface supercells (Figure S1b and c) 16 PETN molecules were arranged in the four molecular layers. The $20 \AA$ of vacuum was added in (110) direction to avoid any interactions between periodically repeated fragments.

Calculations of the pristine PETN surface and surface containing the light absorbing molecule were carried out using $\Gamma$-point only, with a kinetic energy cut-off of $520 \mathrm{eV}$. The convergence criterion for electronic steps was set to $10^{-5} \mathrm{eV}$, and the maximum force acting on any atom was set not to exceed $0.03 \mathrm{eV} / \AA$.
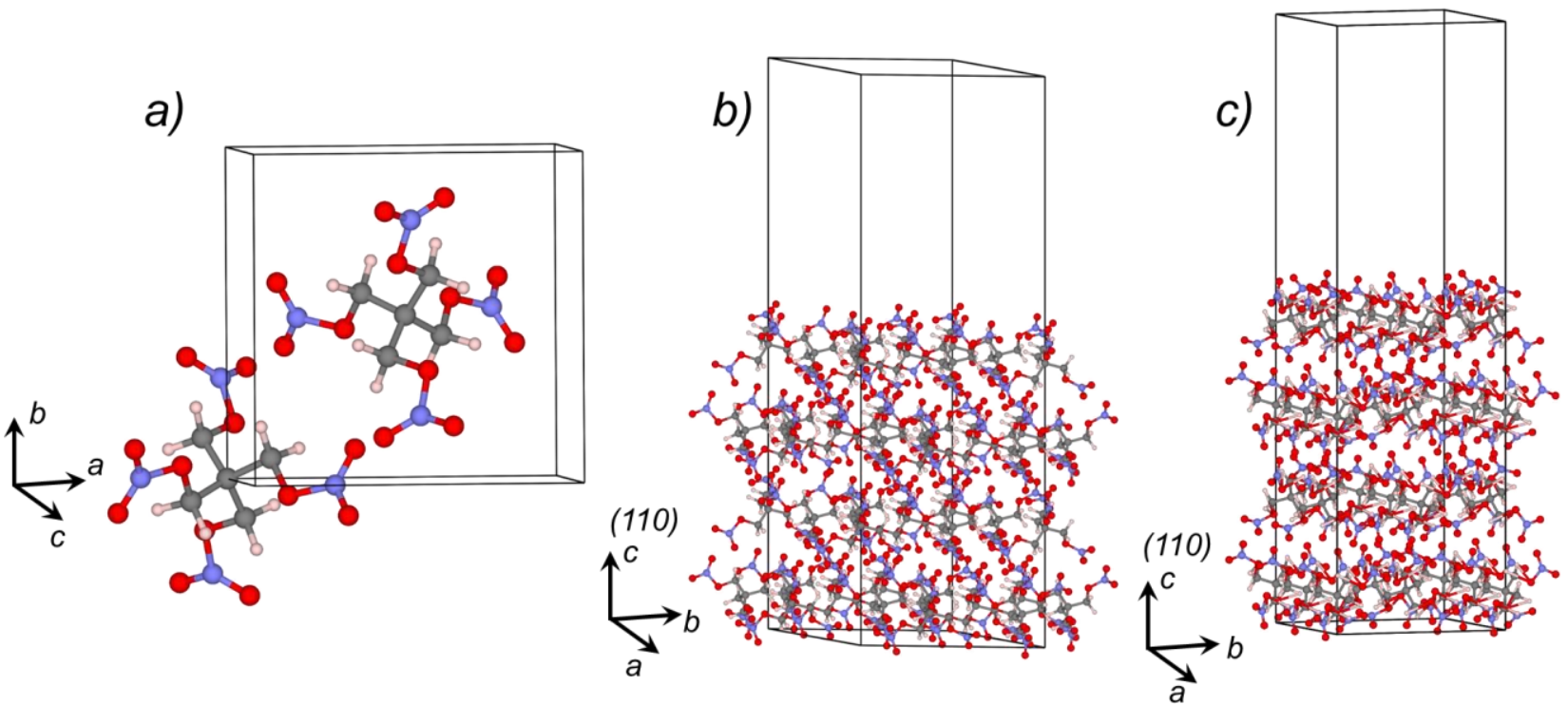

Figure S1. The structures of a) PETN crystal, b) slab model of (110) PETN surface employed for calculations using optPBE-vdW functional, c) slab model of (110) PETN surface employed for calculations using PBE. 


\section{The vertical electronic excitations in the PETN molecule}

The detailed discussion on vertical optical transitions of molecular and crystalline PETN and effect of various organic impurities on the energies the transitions are published in ref. ${ }^{13,14}$.

In accordance with the calculations, optical transitions of PETN molecule observed in the experimental range of 3.18-6.79 $\mathrm{eV}^{15,16,17}$ are localized on $\mathrm{ONO}_{2}$ fragments, which is quite similar to the ethyl nitrate case.

Two lowest optical transitions in a PETN molecule correspond to the singlet-triplet excitations $\mathrm{S}_{0} \rightarrow \mathrm{T}_{1}$ and $\mathrm{S}_{0} \rightarrow \mathrm{T}_{2}$ with energies of 3.88 and $4.18 \mathrm{eV}$, which are close to the experimental estimate of $4.27 \mathrm{eV} .{ }^{15}$ The magnitude of the oscillator strength indicates a low-intensity transition, which is consistent with the nature of a spin-forbidden singlet-triplet excitation, and the broad band in UV absorption spectra of PETN may be explained by an overlap of these two transitions.

The lowest singlet-singlet $\mathrm{S}_{0} \rightarrow \mathrm{S}_{1}\left(n_{O} \rightarrow \pi^{*}\right.$, Figure $\left.\mathrm{S} 2\right)$ excitation with the energy of $4.76 \mathrm{eV}$ matches up with the second absorption peak at $4.77-4.8 \mathrm{eV}^{15,16,17}$. Further, our calculations predict that the singlet-singlet $S_{0} \rightarrow S_{1}$ transition is followed by a pair of spin-forbidden $S_{0} \rightarrow T_{3}(5.20 \mathrm{eV})$ and $\mathrm{S}_{0} \rightarrow \mathrm{T}_{4}(5.32 \mathrm{eV})$ transitions assigned as $n \sigma \rightarrow \pi^{*}$ and $n \pi \rightarrow \pi^{*}$ (Figure $\mathrm{S} 2$ ). We note that neither those two singlet-triplet transitions nor the next singlet-singlet excitation (also obtained theoretically) appear as well-resolved individual peaks in the reported experiments. Nevertheless, the close energy $(5.66 \mathrm{eV})$ of the next transition, $\mathrm{S}_{0} \rightarrow \mathrm{S}_{2}$, and the small non-zero oscillator strength $\left(5 \times 10^{-4}\right)$ indicate a low intensity transition and possible overlap with the adjacent maxima, consistent with the most recent measurements ${ }^{17}$ which show that PETN exhibits absorption at the energies somewhat higher than $5.28 \mathrm{eV}$. According to recent laser-irradiation experiments ${ }^{16}$, gasphase PETN can be excited by absorption of a single UV photon at wavelengths of $236(5.25 \mathrm{eV})$ and $226(5.48 \mathrm{eV}) \mathrm{nm}$. A similarity with the pattern in the EN spectrum lends some additional support to the interpretation of these excitations.

The next calculated excitation is a singlet-singlet $\mathrm{S}_{0} \rightarrow \mathrm{S}_{3}\left(\mathrm{n} \pi \rightarrow \pi^{*}\right.$, Figure $\left.\mathrm{S} 2\right)$ transition with an energy of $6.07 \mathrm{eV}$ and a pronounced oscillator strength of $f=0.1181$, indicating a high intensity absorption maximum and nicely fitting the experimental result of a broad, strong absorption peak at or above $\sim 6.41-6.52 \mathrm{eV}^{15,16,17}$. This high intensity $\mathrm{S}_{0} \rightarrow \mathrm{S}_{3}$ transition is followed by two singlet-singlet, $\mathrm{S}_{0} \rightarrow \mathrm{S}_{4}\left(\mathrm{n}_{\mathrm{O}} \rightarrow \pi^{*}\right.$, Figure $\left.\mathrm{S} 2\right)$ and $\mathrm{S}_{0} \rightarrow \mathrm{S}_{5}\left(\mathrm{n} \pi \rightarrow \pi^{*}\right.$, Figure $\left.\mathrm{S} 2\right)$, transitions at 6.21 and $6.44 \mathrm{eV}$, respectively. Their oscillator strengths (0.0027 and 0.0290) define relatively intense transitions that are likely to contribute to the breadth of the main absorption maximum. 

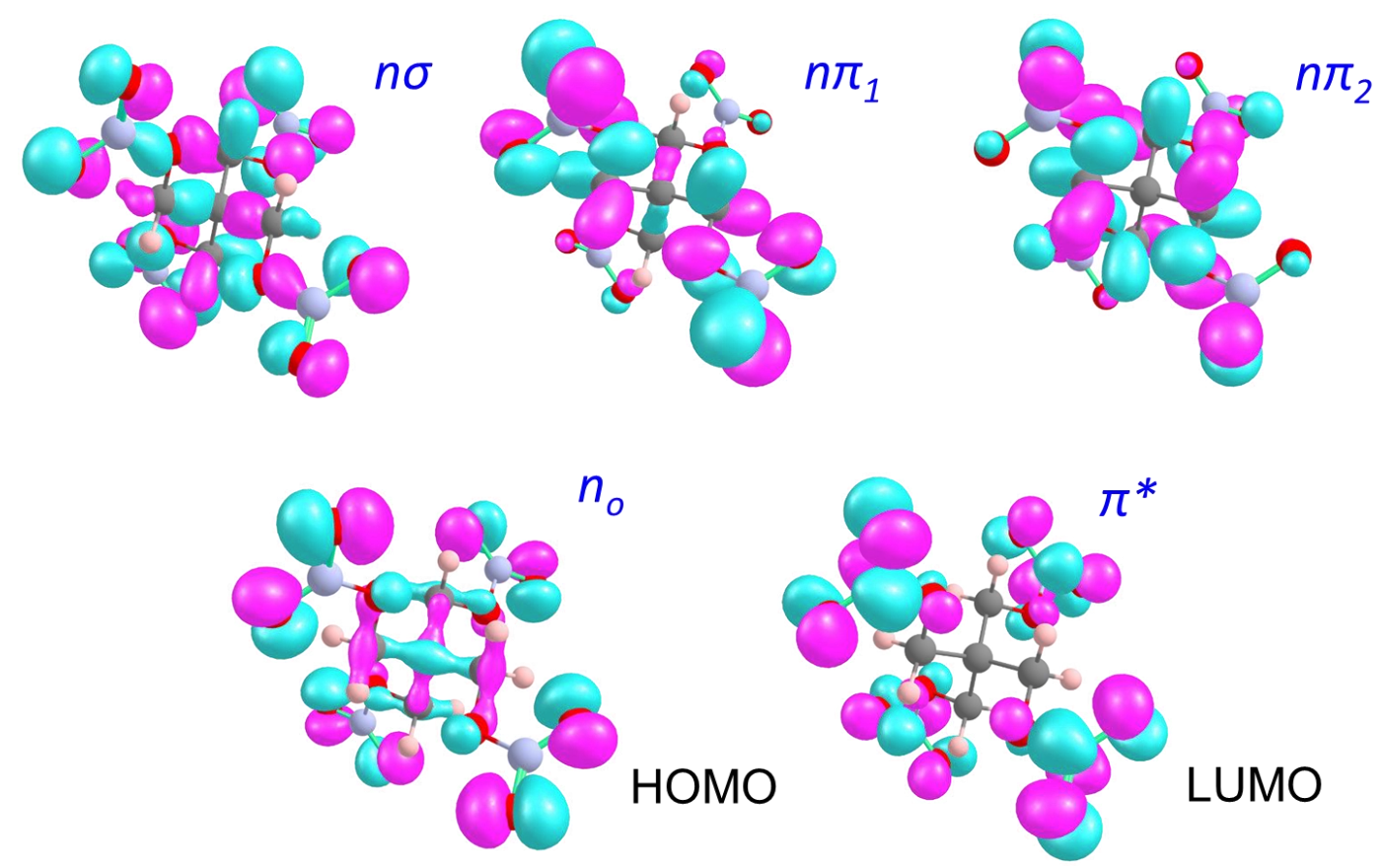

Figure S2. Molecular orbitals of PETN involved in the configuration interaction procedure to simulate excited states.

\section{The vertical electronic excitations in the PQ molecule}

Transitions in PQ molecule in the energy range below $4 \mathrm{eV}$ correspond to promotion of electron from HOMO-3, HOMO-2, HOMO-1 and HOMO to LUMO orbitals (Figure S3). The most intense transition corresponds to $\left(\pi \rightarrow \pi^{*}\right)$ singlet-singlet transition and associated with promotion of electron from HOMO-2 to LUMO (Figure S3). The energy of this transition is $4.07 \mathrm{eV}$ which is in good agreement with the energy of experimentally registered maximum at $3.97 \mathrm{eV}^{18}$. The next singlet-singlet $\left(\pi \rightarrow \pi^{*}\right)$ transition with the energy of $3.81 \mathrm{eV}$ lies within the same intense and broad absorption band and corresponds to promotion of electron from HOMO-2 to LUMO of PQ (Figure $\mathrm{S} 3)$. Vertical transition at $3.03 \mathrm{eV}$ corresponds $\left(\pi \rightarrow \pi^{*}\right)$ singlet-singlet transition and associated with excitation of electron from HOMO to LUMO of PQ (Figure S3). Calculated energy is in good agreement with experimental value of $3.0 \mathrm{eV}^{18}$. The calculated energy of the symmetry forbidden $(n$ $\left.\rightarrow \pi^{*}\right) \mathrm{S}_{0} \rightarrow \mathrm{S}_{1}$ transition $2.31 \mathrm{eV}$ is consistent with the position of weak shoulder in the absorption spectrum of $\mathrm{PQ}$ at $2.48 \mathrm{eV}^{18}$. Further, two low energy vertical transitions in PQ molecule are singlet-triplet transitions $\mathrm{S}_{0} \rightarrow T_{2}{ }^{3}\left(\pi \rightarrow \pi^{*}\right)$ and $\mathrm{S}_{0} \rightarrow T_{1}{ }^{3}\left(n \rightarrow \pi^{*}\right)$ with the energies of 2.25 and 1.78 
$\mathrm{eV}$, respectively. These energies are consistent with the experimentally measured energy of the ${ }^{3}\left(n \rightarrow \pi^{*}\right)$ transition at $2.16 \mathrm{eV}^{18}$.

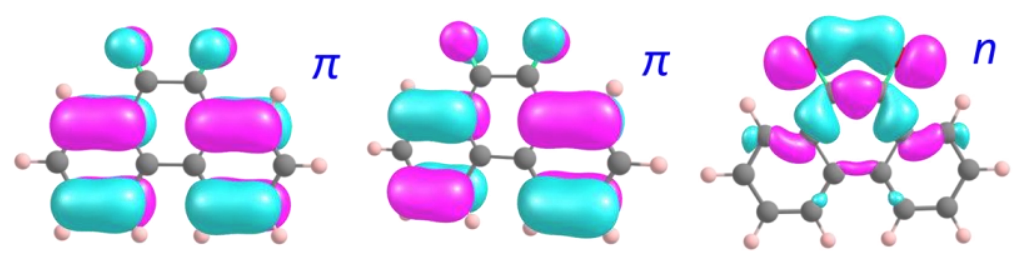

HOMO-3 HOMO-2 HOMO-1

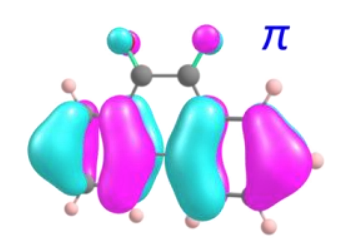

HOMO

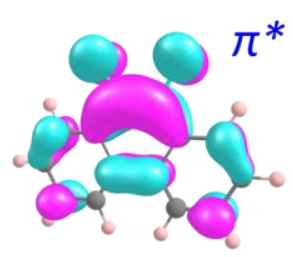

LUMO

Figure S3. Isosurfaces of frontier molecular orbitals of PQ.

\section{The vertical electronic excitations in the BQ molecule}

The calculated using TD PBE0 energy of the lowest vertical ${ }^{3}\left(n \rightarrow \pi^{*}\right)$ singlet-triplet transition corresponding to $\mathrm{HOMO} \rightarrow \mathrm{LUMO}$ excitation is $1.30 \mathrm{eV}$. The second vertical singlettriplet transition requires $1.39 \mathrm{eV}$ and corresponds to HOMO- $1 \rightarrow$ LUMO $\left(\pi \rightarrow \pi^{*}\right.$, Figure S4) excitation. There is no experimental evidence of the two lowest triplet transitions. Most likely the lack of data is due to high reactivity and low chemical stability of $\mathrm{BQ}^{19,20}$. The energy of the $\mathrm{S}_{0} \rightarrow \mathrm{S}_{1}$ singlet-singlet $n \rightarrow \pi^{*}$ (HOMO $\rightarrow$ LUMO, Figure S4) symmetry forbidden transition of $1.84 \mathrm{eV}$ is consistent with the energy of the lowest experimentally registered optical transition at $1.85 \mathrm{eV}$ corresponding to the weak and broad band in the origin of BQ absorption spectrum ${ }^{18}$. The $S_{0} \rightarrow S_{1}$ transition is followed by the series of the singlet-singlet $\pi \rightarrow \pi^{*}$ excitations with calculated energies of 3.19 (HOMO-1 $\rightarrow$ LUMO, Figure S4), 5.57 (HOMO-3 $\rightarrow$ LUMO, Figure S4) and 6.40 (HOMO$4 \rightarrow$ LUMO + HOMO-1 $\rightarrow$ LUMO+1, Figure S4) eV. The calculated using TD PBE0 energies of vertical transitions were found in good agreement with the energies of experimentally observed transitions of intermediate intensity at $3.18^{20}-3.26^{18} \mathrm{eV}$ and $\sim 5 \mathrm{eV}^{18,20}$ and a strong band with a maximum at $\sim 6.20 \mathrm{eV}^{18,20}$. 


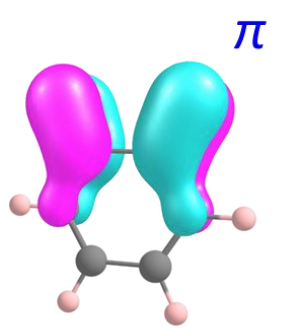

HOMO-4

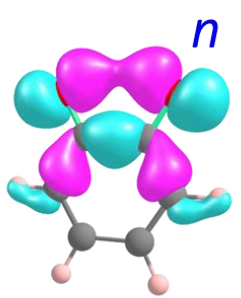

HOMO

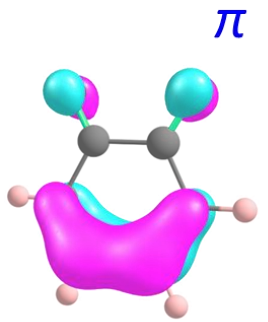

HOMO-3

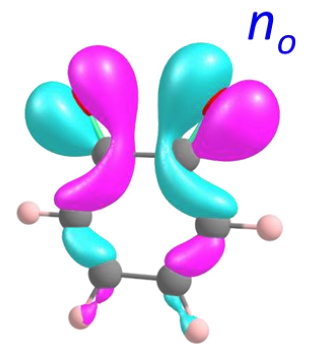

HOMO-2

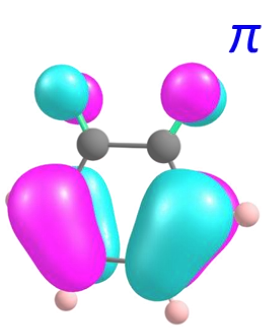

HOMO-1

Figure S4. Isosurfaces of frontier molecular orbitals of BQ.

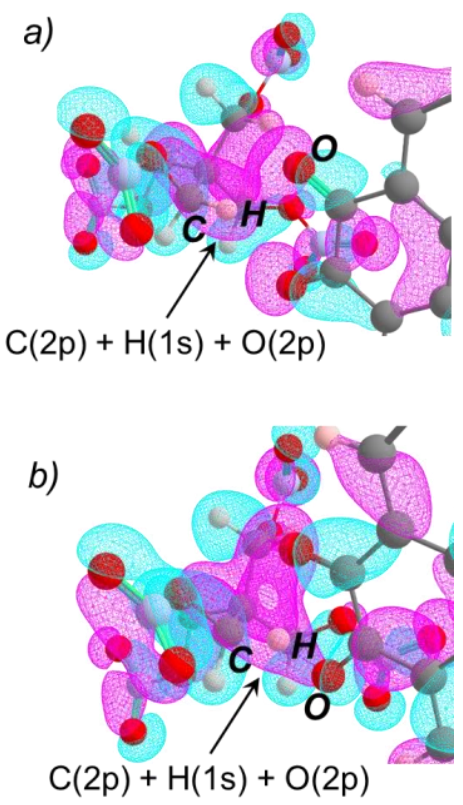

Figure S5. The overlap between $\mathrm{C}(2 \mathrm{p})$ and $\mathrm{H}(1 \mathrm{~s})$ atomic functions of PETN and $\mathrm{O}(2 \mathrm{p})$ functions of PQ in a) the ${ }^{3}\left(n, \pi^{*}\right)$ triplet configuration of PQ-PETN complex and b) the transition state structure of the hydrogen abstraction reaction. 
a)

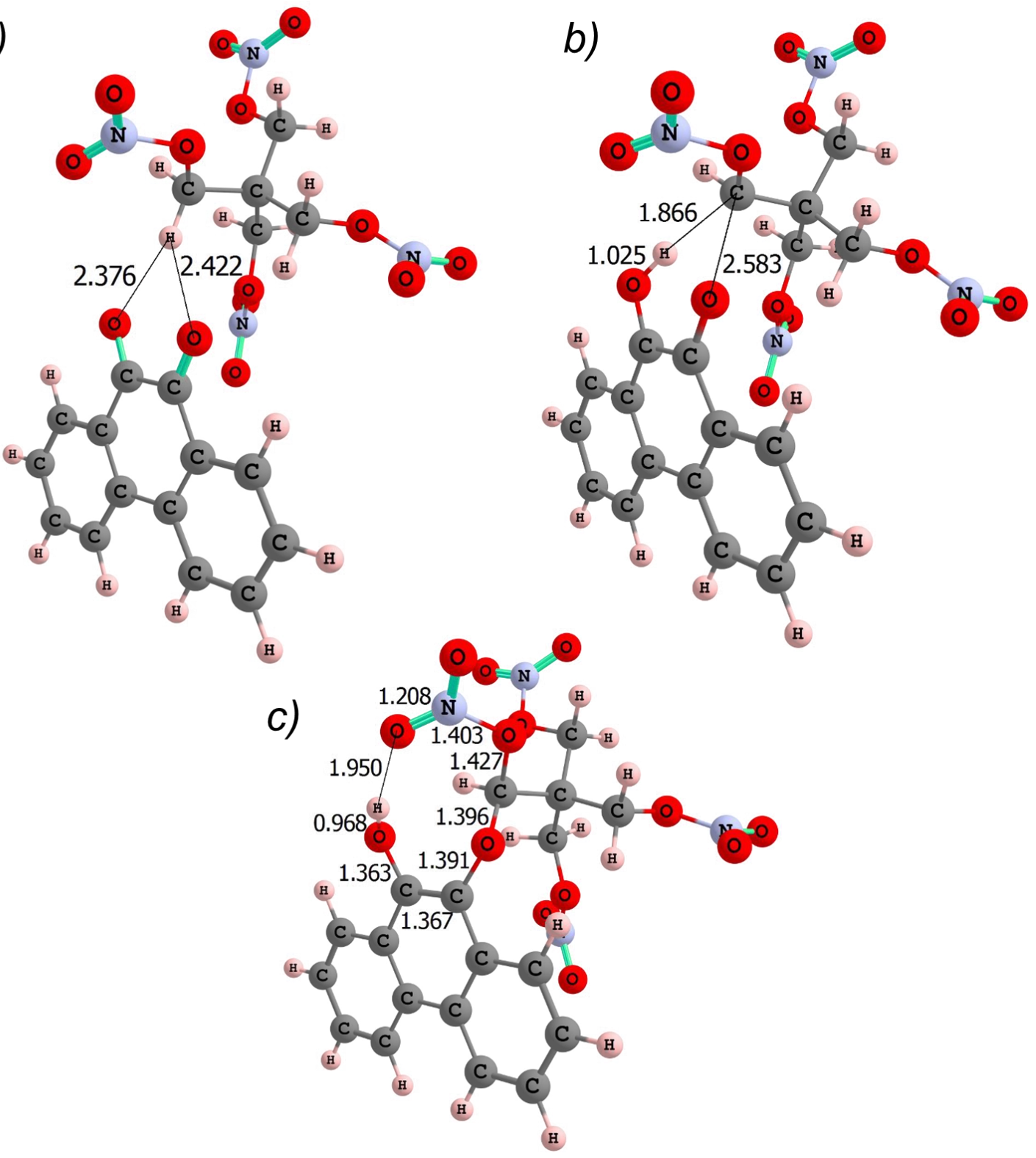

Figure S6. Molecular structures of the a) $\mathrm{S}_{0}$ ground state PQ-PETN molecular complex, b) Transition state of hydrogen transfer reaction on $S_{0}$ potential energy surface, and c) product of the hydrogen transfer reaction. 

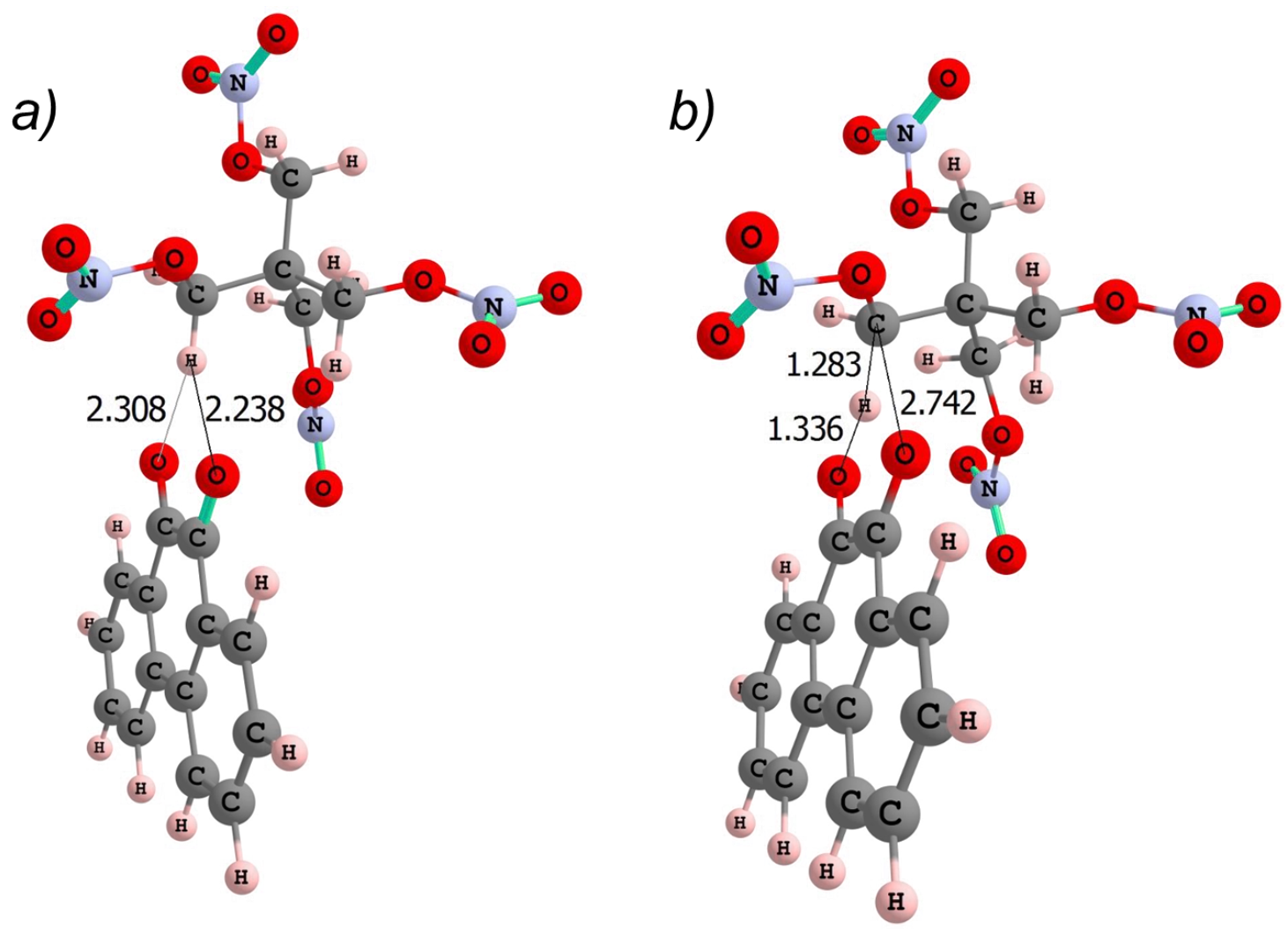

Figure S7. Calculated using $\triangle \mathrm{SCF}$ approach molecular structures of the a) PQ-PETN molecular complex on $\mathrm{T} 1$ surface and b) transition state of hydrogen transfer reaction on $\mathrm{T} 1$ potential energy surface. 
a)
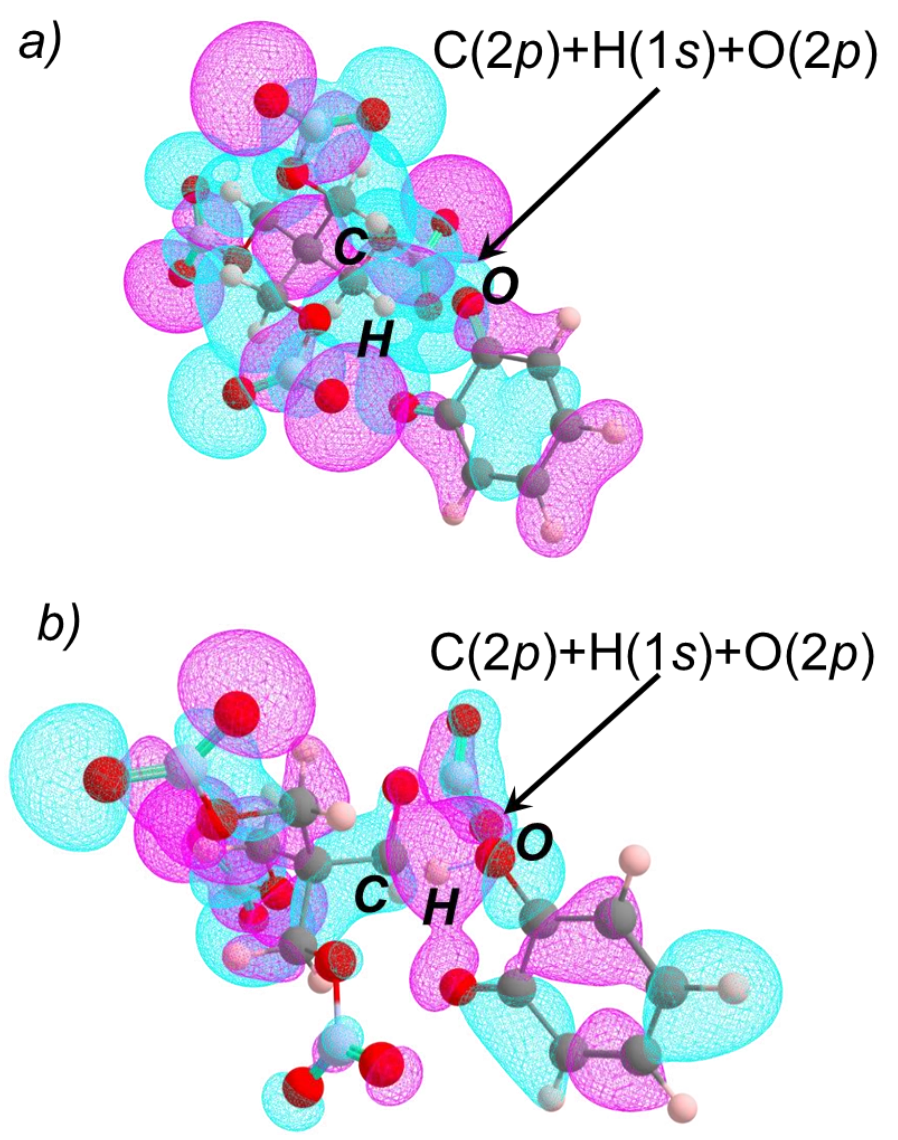

Figure S8. The overlap between C (2p) and H (1s) atomic functions of PETN and O (2p) functions of BQ in a) the ${ }^{3}\left(n, \pi^{*}\right)$ triplet configuration of BQ-PETN complex and b) the TS structure of the hydrogen abstraction reaction. 


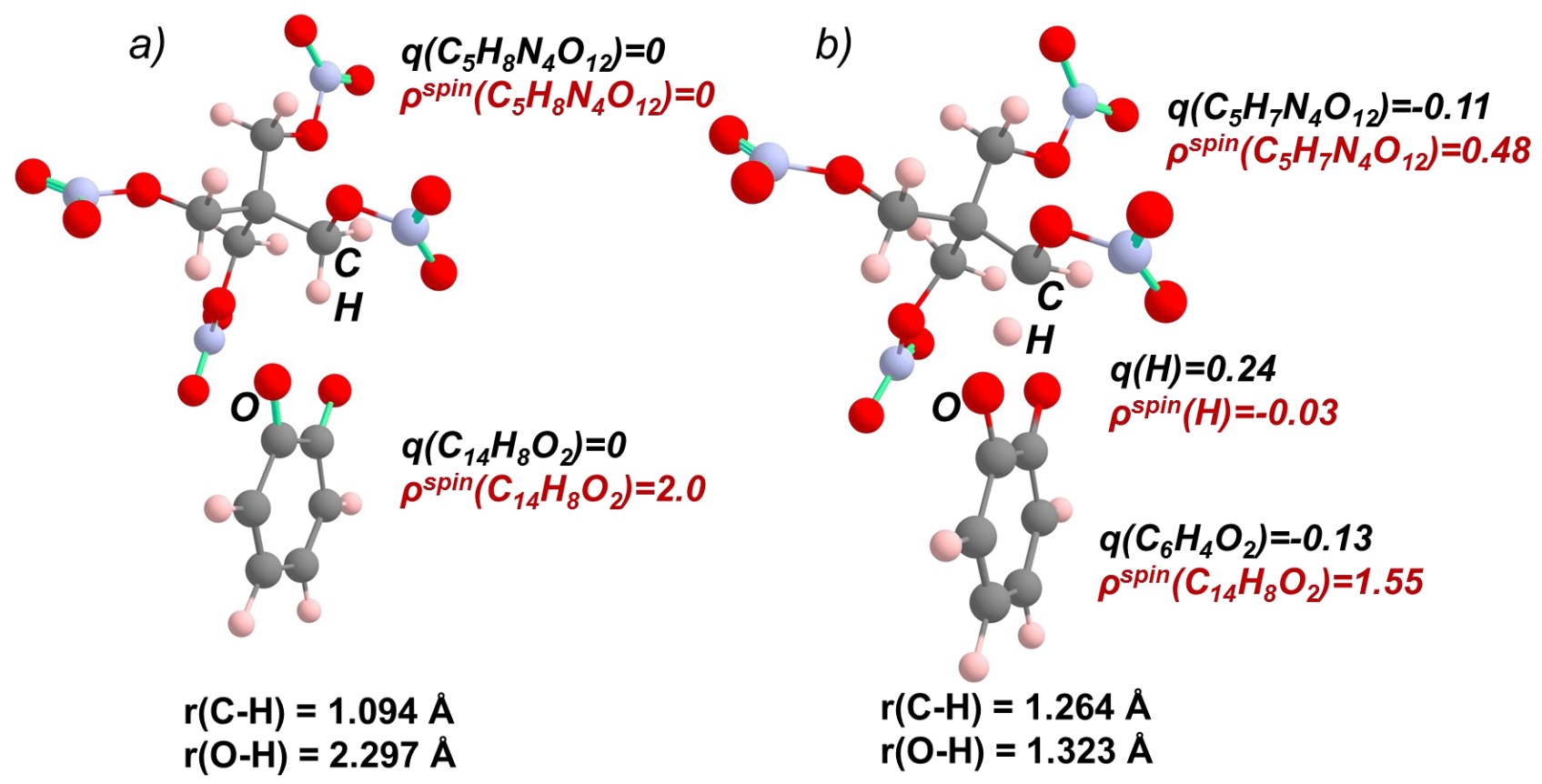

Figure S9. The Mulliken atomic charges and spin densities in a) equilibrium structure of triplet PQPETN complex and b) transition state structure of hydrogen abstraction reaction. 
PQ (ground state configuration):

\section{Coordinates}

\section{Method: PBE0}

$\begin{array}{lrrr}6 & -4.827730000 & -6.227739000 & -0.010376000 \\ 6 & -3.441291000 & -6.236479000 & -0.011610000 \\ 6 & -2.720648000 & -5.037752000 & -0.010163000 \\ 6 & -3.384717000 & -3.792352000 & -0.007428000 \\ 6 & -4.786285000 & -3.808302000 & -0.006221000 \\ 6 & -5.496516000 & -5.003521000 & -0.007664000 \\ 6 & -1.243818000 & -5.124714000 & -0.011596000 \\ 6 & -2.617297000 & -2.526070000 & -0.005917000 \\ 6 & -1.205960000 & -2.538412000 & -0.007158000 \\ 6 & -0.445445000 & -3.807338000 & -0.009994000 \\ 6 & -0.476823000 & -1.344828000 & -0.005735000 \\ 1 & 0.606862000 & -1.413821000 & -0.006787000 \\ 6 & -1.126192000 & -0.119829000 & -0.003072000 \\ 6 & -2.520926000 & -0.093510000 & -0.001824000 \\ 6 & -3.251771000 & -1.276233000 & -0.003223000 \\ 1 & -5.384441000 & -7.159811000 & -0.011503000 \\ 1 & -2.878645000 & -7.165217000 & -0.013715000 \\ 1 & -5.346097000 & -2.880141000 & -0.004118000 \\ 1 & -6.582548000 & -4.976427000 & -0.006659000 \\ 1 & -0.557550000 & 0.805012000 & -0.001975000 \\ 1 & -3.047524000 & 0.856693000 & 0.000260000 \\ 1 & -4.333633000 & -1.209489000 & -0.002177000 \\ 8 & 0.767563000 & -3.850623000 & -0.011096000 \\ 8 & -0.644103000 & -6.179971000 & -0.013951000\end{array}$




\begin{tabular}{|c|c|c|c|}
\hline \\
\hline & & & Method: PBEO \\
\hline \multirow{2}{*}{\multicolumn{4}{|c|}{$\begin{array}{llrl}6 & 0.835545000 & -0.464359000 & 0.305540000 \\
6 & 1.569148000 & 0.308315000 & 1.192503000\end{array}$}} \\
\hline & & & \\
\hline & \multicolumn{3}{|c|}{$\begin{array}{ccc}0.835545000 & -0.464359000 & 0.305540000 \\
1.569148000 & 0.308315000 & 1.192503000 \\
2.928612000 & 0.549643000 & 0.969076000\end{array}$} \\
\hline & 000 & 98000 & 6344600 \\
\hline & 2.824107000 & 79000 & 5600 \\
\hline & 1.473392000 & & -0 . \\
\hline & 3.65 & & 1 \\
\hline & & & \\
\hline & & & \\
\hline & 000 & & \\
\hline & 7.147097000 & 2000 & 0.332 \\
\hline & & 6000 & 89000 \\
\hline & & & 200 \\
\hline & & & \\
\hline & & & \\
\hline & -0.2 & & \\
\hline & & & \\
\hline & 3.2 & 00 & 5000 \\
\hline & & & \\
\hline & & & \\
\hline & & & \\
\hline & & & \\
\hline & & & \\
\hline & & & \\
\hline & & & 00 \\
\hline & & & 000 \\
\hline & & & \\
\hline & & & \\
\hline & & & \\
\hline & & & \\
\hline & & & \\
\hline & & & \\
\hline & & & \\
\hline & & & \\
\hline & & & 000 \\
\hline & & & 3000 \\
\hline & & & \\
\hline & & & \\
\hline & & & \\
\hline & & & \\
\hline & & & 000 \\
\hline & & & \\
\hline & 4.12 & 000 & 4000 \\
\hline & 3.3 & 000 & 2000 \\
\hline & 8.5 & 4000 & 59000 \\
\hline & & & \\
\hline & & & \\
\hline & & & \\
\hline & & & \\
\hline & & & 83000 \\
\hline & 5.58 & 97000 & 5.897922000 \\
\hline & 2.529 & 8.049701000 & 7.002015000 \\
\hline & 6.249775000 & 2.558669000 & 7.443690000 \\
\hline
\end{tabular}




\begin{tabular}{|c|c|c|c|}
\hline \multicolumn{4}{|c|}{$\begin{array}{l}\text { PQ-PETN }\left(T_{1},{ }^{3}\left(n, \pi^{*}\right) \text { state): }\right. \\
\text { Method: TDPBE0 }\end{array}$} \\
\hline & 0.806007000 & -0.296367000 & 0.24550200 \\
\hline & 376000 & 7000 & 70000 \\
\hline & 2.940665000 & 0.668523000 & 0.854386000 \\
\hline & 118000 & -0.072746000 & 500 \\
\hline & 2.807162000 & -0.920485000 & -0.954478000 \\
\hline & 1.441553000 & 4665000 & 311000 \\
\hline & 3.649983000 & 1.585337000 & 1.736618000 \\
\hline & 5.035373000 & 0.056835000 & -0.329543000 \\
\hline & 5.778702000 & 0.871387000 & 97000 \\
\hline & 000 & & 9000 \\
\hline & 7.169467000 & & \\
\hline & 8000 & & \\
\hline & 9000 & & 5000 \\
\hline & 2000 & 00 & 5000 \\
\hline & 7000 & 000 & \\
\hline & 3000 & & \\
\hline & & & \\
\hline & 000 & & -1 \\
\hline & & & \\
\hline & 000 & & 000 \\
\hline & 000 & & 000 \\
\hline & 000 & & 3000 \\
\hline & & & \\
\hline & & & 2 \\
\hline & 5.7 & & 000 \\
\hline & & & \\
\hline & & & \\
\hline & & & \\
\hline & 00 & & 00 \\
\hline & & & 2000 \\
\hline & & & 7000 \\
\hline & 000 & & 000 \\
\hline & & & 000 \\
\hline & & & \\
\hline & & & \\
\hline & & & \\
\hline & 00 & & 000 \\
\hline & & & \\
\hline & 000 & & 0000 \\
\hline & 3.5 & & 8000 \\
\hline . & 000 & & 0000 \\
\hline & & & \\
\hline & & & \\
\hline & & & \\
\hline & & & \\
\hline & & & \\
\hline & & & 6000 \\
\hline$p$ & 4000 & 000 & 0000 \\
\hline & 2000 & 000 & 795000 \\
\hline & 4.5 & & \\
\hline & & & \\
\hline & & & \\
\hline & & 2.315403000 & 6.9395180 \\
\hline
\end{tabular}




\begin{tabular}{|c|c|c|c|}
\hline \multicolumn{4}{|c|}{$\begin{array}{l}\text { PQ-PETN }\left(T_{1},{ }^{3}\left(n, \pi^{*}\right) \text { state): }\right. \\
\text { Method: PBE0, } \Delta \text { SCF }\end{array}$} \\
\hline & 0.797465000 & -0.055519000 & 0.070817000 \\
\hline & 1.558357000 & 0.736582000 & 0.906706000 \\
\hline & 2.964339000 & 0.742468000 & 0.797898000 \\
\hline & 3.614497000 & -0.058265000 & -0.163981000 \\
\hline & 2.810322000 & -0.852917000 & -0.999338000 \\
\hline & 1.430079000 & -0.855368000 & -0.888357000 \\
\hline & 3.685 & 1.610311000 & 1.721285000 \\
\hline & 5.069551000 & -0.037631000 & -0.261823000 \\
\hline & $5.81 \mathrm{c}$ & & 000 \\
\hline & 5.20 & & \\
\hline & 5000 & & 7000 \\
\hline & 68000 & 000 & 000 \\
\hline & 90000 & 3000 & -0.403046000 \\
\hline & 7.15 & -0.7 & 200 \\
\hline & 5.77 & 6000 & 7000 \\
\hline & $-0.2 \varepsilon$ & & \\
\hline & & & \\
\hline & & & \\
\hline & 0.8 & -1. & 1000 \\
\hline & $8.9^{7}$ & & 1000 \\
\hline & $7.6^{7}$ & & -2 \\
\hline & 5.23 & & 0000 \\
\hline & & & \\
\hline & & & \\
\hline & & & \\
\hline & & & \\
\hline & 3.8 & 00 & 000 \\
\hline & & & \\
\hline & & & 000 \\
\hline & & & 000 \\
\hline & & & \\
\hline & & & \\
\hline & & & \\
\hline & 6.3 & & 000 \\
\hline & 5.02 & & \\
\hline & & & 000 \\
\hline & & 00 & 3000 \\
\hline & & & 000 \\
\hline & & & \\
\hline & & & \\
\hline & & & \\
\hline & & & 000 \\
\hline & & & \\
\hline & & & 0000 \\
\hline & 8.31 & 000 & 7000 \\
\hline & 6.40 & 00 & 000 \\
\hline & & & \\
\hline & & & \\
\hline & & & \\
\hline & & & $50 ?$ \\
\hline & 2000 & 3.846314000 & 5.490152000 \\
\hline & 2.530237000 & 7.392128000 & 7.543787000 \\
\hline & 6.837130000 & 2.292902000 & 6.64706200 \\
\hline
\end{tabular}




\begin{tabular}{|c|c|c|c|}
\hline \multicolumn{4}{|c|}{$\begin{array}{l}\text { PQ-PETN }\left(\mathrm{T}_{2},{ }^{3}\left(\pi, \pi^{*}\right) \text { state }\right) \text { : } \\
\text { Method: TDPBE0 }\end{array}$} \\
\hline & 8506000 & -0.035804000 & 0.066 \\
\hline & 1.603356000 & 1000 & 7000 \\
\hline & 3.003191000 & 0.743926000 & 0.869235000 \\
\hline & 3.672779000 & 0.027678000 & -0.210040000 \\
\hline & 2.856064000 & -0.711029000 & -1.127515000 \\
\hline & 1.496471000 & -0.743810000 & -0.996577000 \\
\hline & 3.731624000 & 1.530175000 & 1.853499000 \\
\hline & 5.081066000 & 086046000 & -0.312592000 \\
\hline & 529000 & 7539000 & 3679000 \\
\hline & 1000 & & 6000 \\
\hline & 933000 & & 000 \\
\hline & 5000 & & \\
\hline & 8000 & & 0000 \\
\hline & 2000 & & 2000 \\
\hline & 8000 & & \\
\hline & 9000 & & \\
\hline & 000 & & \\
\hline & 5000 & & \\
\hline & & & \\
\hline & 000 & & \\
\hline & 000 & & 000 \\
\hline & 000 & & 0000 \\
\hline & & & \\
\hline & 000 & & 000 \\
\hline & & & 000 \\
\hline & & & \\
\hline & & & \\
\hline & & & \\
\hline & & & \\
\hline & & & 3000 \\
\hline & & & 5000 \\
\hline 1 & 000 & & 000 \\
\hline & & & 2000 \\
\hline & & & \\
\hline & & & \\
\hline & & & \\
\hline & 00 & & 000 \\
\hline & & & 8000 \\
\hline & & & \\
\hline & 000 & & 000 \\
\hline . & 000 & & 9000 \\
\hline & & & 000 \\
\hline & & & \\
\hline & & & \\
\hline & & & \\
\hline & & & \\
\hline & & & 1000 \\
\hline$p$ & 000 & & 00000 \\
\hline ? & 2000 & 000 & 04000 \\
\hline & 4.7 & & 4000 \\
\hline & & & \\
\hline & & & \\
\hline & & 2.413531000 & 6.9272310 \\
\hline
\end{tabular}




\begin{tabular}{|c|c|c|c|}
\hline \multicolumn{4}{|c|}{$\begin{array}{l}\text { PQ-PETN }\left(S_{1},{ }^{1}\left(n, \pi^{*}\right) \text { state }\right): \\
\text { Method: TDPBE0 }\end{array}$} \\
\hline & 4999000 & -0.007830000 & 0.06280800 \\
\hline & 7645000 & 85000 & 5000 \\
\hline & 2.975811000 & 0.768132000 & 0.803184000 \\
\hline & 3.626107000 & -0.054255000 & -0.142440000 \\
\hline & 2.819018000 & -0.846901000 & -0.975709000 \\
\hline & 1.437209000 & -0.828253000 & -0.879464000 \\
\hline & 3.695593000 & 1.635952000 & 1.723119000 \\
\hline & 5.081404000 & -0.055008000 & -0.224918000 \\
\hline & 37000 & 0.765828000 & 3256000 \\
\hline & 4000 & 7000 & 50000 \\
\hline & 7.244628000 & & 57000 \\
\hline & 7000 & & 4000 \\
\hline & 000 & & 3000 \\
\hline & 1000 & 6000 & 0000 \\
\hline & 000 & & 7000 \\
\hline & -0.2 & & \\
\hline & & & \\
\hline & & & -1 \\
\hline 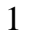 & & & \\
\hline & & & 000 \\
\hline & 000 & & 000 \\
\hline & 00 & & 6000 \\
\hline & & & \\
\hline & & & 5000 \\
\hline & & & 000 \\
\hline & & & \\
\hline 1 & & & \\
\hline & & & \\
\hline & & & \\
\hline & & & 000 \\
\hline & & & \\
\hline & & & 5000 \\
\hline & & & 0000 \\
\hline & & & \\
\hline & & & \\
\hline & & & \\
\hline & & & 000 \\
\hline & & & 5000 \\
\hline & & & 5000 \\
\hline & 000 & & 000 \\
\hline . & & & 6000 \\
\hline & & & \\
\hline & & & \\
\hline & & & \\
\hline & & & \\
\hline & & & \\
\hline & & & \\
\hline$p$ & 4.5 & & 84000 \\
\hline 3 & 10000 & 9000 & 92000 \\
\hline 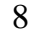 & 4.9 & & 000 \\
\hline & & & \\
\hline & & & \\
\hline & 6.916130000 & 2.264124000 & 6.5101330 \\
\hline
\end{tabular}




\begin{tabular}{|c|c|c|c|}
\hline \multicolumn{4}{|c|}{$\begin{array}{l}\text { PQ-PETN }\left(S_{2},{ }^{1}\left(\pi, \pi^{*}\right) \text { state }\right) \text { : } \\
\text { Method: TDPBE0 }\end{array}$} \\
\hline & 0.877150000 & 0.080037000 & 0.00775400 \\
\hline & 78000 & 15000 & 5000 \\
\hline & 3.017573000 & 814713000 & 0.864661000 \\
\hline & 3.674851000 & 0.089414000 & -0.188685000 \\
\hline & 2.870858000 & -0.631130000 & -1.128051000 \\
\hline & 1.501841000 & -0.636794000 & 3149000 \\
\hline & 3.752875000 & 1.608003000 & 891978000 \\
\hline & 5.098499000 & 0.110748000 & -0.265186000 \\
\hline & 5.842891000 & 0.856407000 & 93000 \\
\hline & 19000 & 8000 & 5000 \\
\hline & 673000 & & \\
\hline & 9000 & & 000 \\
\hline & 4000 & & 3000 \\
\hline & 5000 & & 000 \\
\hline & 2000 & 00 & \\
\hline & 74000 & & \\
\hline & 3000 & & \\
\hline & 2000 & & -1 \\
\hline 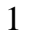 & & & \\
\hline 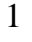 & 000 & & \\
\hline & 000 & & 000 \\
\hline & 000 & & 6000 \\
\hline & & & \\
\hline & & & 7000 \\
\hline & 5000 & & 5 \\
\hline & & & \\
\hline 1 & & & \\
\hline 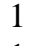 & & & \\
\hline & 3. & & \\
\hline & 000 & & 000 \\
\hline & & & \\
\hline & 000 & & 4.1 \\
\hline & & & 000 \\
\hline & & & \\
\hline & & & \\
\hline & & & \\
\hline & 000 & & 00 \\
\hline & 000 & & 000 \\
\hline & 3000 & & 000 \\
\hline & 0000 & & 000 \\
\hline . & 000 & & 9000 \\
\hline & & & \\
\hline & & & \\
\hline & & & \\
\hline & & & \\
\hline & & & \\
\hline & 000 & & 2000 \\
\hline$p$ & 99000 & 000 & 92000 \\
\hline 3 & 65000 & 000 & 3000 \\
\hline 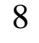 & 4.86 & & \\
\hline & & & \\
\hline & & & \\
\hline & & 2.407276000 & 6.83500100 \\
\hline
\end{tabular}


TS structure of hydrogen abstraction reaction in PQ-PETN $\left(\mathrm{T}_{1},{ }^{3}\left(\mathrm{n}, \pi^{*}\right)\right.$ state) complex: Method: PBE0, $\triangle$ SCF

$\begin{array}{lrrr}6 & 0.855140000 & 0.176386000 & 0.098954000 \\ 6 & 1.649282000 & 0.955254000 & 0.917281000 \\ 6 & 3.054691000 & 0.876119000 & 0.837613000 \\ 6 & 3.673699000 & -0.008149000 & -0.082355000 \\ 6 & 2.836856000 & -0.784462000 & -0.899075000 \\ 6 & 1.455435000 & -0.697356000 & -0.815341000 \\ 6 & 3.855166000 & 1.704469000 & 1.703318000 \\ 6 & 5.133941000 & -0.086362000 & -0.152450000 \\ 6 & 5.922978000 & 0.724616000 & 0.694079000 \\ 6 & 5.312831000 & 1.651146000 & 1.641557000 \\ 6 & 7.325325000 & 0.662617000 & 0.644474000 \\ 1 & 7.890720000 & 1.304020000 & 1.312960000 \\ 6 & 7.958369000 & -0.194398000 & -0.236427000 \\ 6 & 7.188952000 & -1.002848000 & -1.080674000 \\ 6 & 5.804634000 & -0.947649000 & -1.037281000 \\ 1 & -0.226446000 & 0.243925000 & 0.164554000 \\ 1 & 1.205828000 & 1.638596000 & 1.633924000 \\ 1 & 3.268285000 & -1.471935000 & -1.617722000 \\ 1 & 0.839912000 & -1.312802000 & -1.464805000 \\ 1 & 9.042609000 & -0.238928000 & -0.272551000 \\ 1 & 7.675032000 & -1.680058000 & -1.776996000 \\ 1 & 5.240940000 & -1.588531000 & -1.705802000 \\ 8 & 5.983406000 & 2.387005000 & 2.396815000 \\ 8 & 3.283267000 & 2.503126000 & 2.535392000 \\ 1 & 5.686204000 & 5.915173000 & 5.663343000 \\ 1 & 6.974174000 & 5.204024000 & 3.635494000 \\ 1 & 3.689935000 & 3.734479000 & 4.844545000 \\ 1 & 2.656967000 & 5.398980000 & 3.344403000 \\ 1 & 3.561428000 & 6.840702000 & 2.828395000 \\ 1 & 5.061308000 & 7.271544000 & 4.685306000 \\ 1 & 6.128282000 & 4.741662000 & 2.144532000 \\ 1 & 4.217843000 & 3.118428000 & 3.265349000 \\ 6 & 3.596089000 & 5.750367000 & 2.912593000 \\ 6 & 6.118389000 & 5.426331000 & 2.993054000 \\ 6 & 4.883511000 & 6.238037000 & 4.994439000 \\ 6 & 4.796683000 & 5.311705000 & 3.767238000 \\ 6 & 4.591403000 & 3.872639000 & 4.233663000 \\ 7 & 2.617449000 & 5.083236000 & 0.849401000 \\ 7 & 7.275988000 & 7.075886000 & 1.733681000 \\ 7 & 3.519033000 & 6.923145000 & 6.816711000 \\ 7 & 5.694897000 & 2.143345000 & 5.415339000 \\ 8 & 1.599468000 & 5.524815000 & 1.321744000 \\ 8 & 3.781539000 & 5.185235000 & 1.612381000 \\ 8 & 2.807368000 & 4.555970000 & -0.209365000 \\ 8 & 8.052224000 & 6.179204000 & 1.522173000 \\ 8 & 6.179136000 & 6.791277000 & 2.550613000 \\ 8 & 7.286866000 & 8.212813000 & 1.354039000 \\ 8 & 4.485223000 & 7.577874000 & 7.117173000 \\ 8 & 3.617734000 & 6.136966000 & 5.660855000 \\ 8 & 4.668977000 & 1.541099000 & 5.259545000 \\ 8 & 5.740801000 & 3.468998000 & 4.901415000 \\ 8 & 2.453821000 & 6.823893000 & 7.353645000 \\ 8 & 6.718487000 & 1.833351000 & 5.946146000\end{array}$


TS structure of hydrogen abstraction reaction in PQ-PETN (ground state) complex: Method: PBE0

$\begin{array}{lrrr}6 & 0.879452000 & 0.204807000 & 0.619800000 \\ 6 & 1.623788000 & 0.736392000 & 1.653460000 \\ 6 & 3.014614000 & 0.915894000 & 1.509015000 \\ 6 & 3.676688000 & 0.566104000 & 0.299545000 \\ 6 & 2.890407000 & 0.018154000 & -0.723273000 \\ 6 & 1.522474000 & -0.159726000 & -0.569913000 \\ 6 & 3.788628000 & 1.423599000 & 2.590719000 \\ 6 & 5.117382000 & 0.807954000 & 0.145940000 \\ 6 & 5.866058000 & 1.355314000 & 1.215619000 \\ 6 & 5.219421000 & 1.621160000 & 2.492592000 \\ 6 & 7.237743000 & 1.609084000 & 1.078359000 \\ 1 & 7.775781000 & 2.015558000 & 1.929484000 \\ 6 & 7.883482000 & 1.340474000 & -0.116517000 \\ 6 & 7.158294000 & 0.793858000 & -1.179404000 \\ 6 & 5.802068000 & 0.529840000 & -1.047489000 \\ 1 & -0.191393000 & 0.065364000 & 0.729386000 \\ 1 & 1.158202000 & 1.013869000 & 2.593853000 \\ 1 & 3.344594000 & -0.271605000 & -1.663754000 \\ 1 & 0.947053000 & -0.585367000 & -1.386866000 \\ 1 & 8.943363000 & 1.549132000 & -0.224731000 \\ 1 & 7.655638000 & 0.572514000 & -2.119216000 \\ 1 & 5.274976000 & 0.107897000 & -1.895815000 \\ 8 & 5.772770000 & 2.130696000 & 3.492332000 \\ 8 & 3.240107000 & 1.875995000 & 3.676446000 \\ 1 & 5.813738000 & 6.359532000 & 5.711580000 \\ 1 & 7.139682000 & 5.032214000 & 4.046403000 \\ 1 & 3.680694000 & 4.074371000 & 5.304154000 \\ 1 & 2.874797000 & 5.517553000 & 3.463998000 \\ 1 & 3.984467000 & 6.535386000 & 2.513713000 \\ 1 & 5.328048000 & 7.436169000 & 4.371355000 \\ 1 & 6.335765000 & 4.270641000 & 2.658456000 \\ 1 & 3.933583000 & 2.458306000 & 4.157005000 \\ 6 & 3.857350000 & 5.558609000 & 2.986801000 \\ 6 & 6.338230000 & 5.142314000 & 3.311543000 \\ 6 & 5.067783000 & 6.533274000 & 4.931188000 \\ 6 & 4.975372000 & 5.298661000 & 4.001964000 \\ 6 & 4.651614000 & 4.056710000 & 4.798679000 \\ 7 & 3.110870000 & 4.726570000 & 0.896142000 \\ 7 & 7.707550000 & 6.347919000 & 1.788809000 \\ 7 & 3.679159000 & 7.739394000 & 6.434699000 \\ 7 & 5.549015000 & 2.631629000 & 6.444518000 \\ 8 & 2.364487000 & 5.672775000 & 0.934213000 \\ 8 & 3.956867000 & 4.530972000 & 1.988794000 \\ 8 & 3.249148000 & 3.890849000 & 0.046595000 \\ 8 & 8.426140000 & 5.383231000 & 1.875559000 \\ 8 & 6.537629000 & 6.343991000 & 2.546238000 \\ 8 & 7.837406000 & 7.347306000 & 1.138811000 \\ 8 & 4.678867000 & 8.384059000 & 6.632352000 \\ 8 & 3.768887000 & 6.686736000 & 5.520065000 \\ 8 & 4.487946000 & 2.063724000 & 6.366575000 \\ 8 & 5.700866000 & 3.790906000 & 5.665718000 \\ 8 & 2.583558000 & 7.851571000 & 6.906564000 \\ 8 & 6.511294000 & 2.396178000 & 7.119690000\end{array}$


Final state (reaction product) of hydrogen abstraction reaction in PQ-PETN (ground state) complex: Method: PBE0

\begin{tabular}{|c|c|c|c|}
\hline 6 & -4.065547000 & -3.565959000 & -1.734250000 \\
\hline & -3.306460000 & -3.019509000 & -0.720238000 \\
\hline & -1.955912000 & -2.679801000 & -0.941889000 \\
\hline O & -1.355943000 & -2.922188000 & -2.204285000 \\
\hline & -2.163655000 & -3.475114000 & -3.219546000 \\
\hline & -3.489507000 & -3.788890000 & -2.994358000 \\
\hline & -1.185115000 & -2.082788000 & 0.116063000 \\
\hline & 0.051193000 & -2.616934000 & -2.392898000 \\
\hline & 0.774080000 & -1.994041000 & -1.341786000 \\
\hline & 0.095764000 & -1.668215000 & -0.118249000 \\
\hline & 2.147603000 & -1.718220000 & -1.504976000 \\
\hline & 2.698028000 & -1.277617000 & -0.681171000 \\
\hline & 2.795742000 & -2.031412000 & -2.681591000 \\
\hline & 2.086088000 & -2.632733000 & -3.731288000 \\
\hline & 0.744325000 & -2.920639000 & -3.582340000 \\
\hline & -5.106632000 & -3.819666000 & -1.557638000 \\
\hline & -3.734640000 & -2.837452000 & 0.259185000 \\
\hline & -1.745228000 & -3.657435000 & -4.203104000 \\
\hline ] & -4.086053000 & -4.211087000 & -3.797814000 \\
\hline & 3.854865000 & -1.817001000 & -2.790354000 \\
\hline ] & 2.591650000 & -2.879746000 & -4.660012000 \\
\hline 1 & 0.220801000 & -3.398987000 & -4.402624000 \\
\hline & 0.875607000 & -1.017810000 & 0.832869000 \\
\hline & -1.828232000 & -1.912086000 & 1.305321000 \\
\hline & 0.894620000 & 2.468071000 & 2.837044000 \\
\hline | & 2.679302000 & 1.546191000 & 1.322666000 \\
\hline & -0.682134000 & -0.088533000 & 1.845226000 \\
\hline . & -1.422274000 & 1.176110000 & -0.008696000 \\
\hline & -0.624264000 & 2.711411000 & -0.406672000 \\
\hline & 0.281995000 & 3.527809000 & 1.543230000 \\
\hline l & 2.246913000 & 0.903700000 & -0.284721000 \\
\hline & -1.208312000 & -2.023533000 & 2.039971000 \\
\hline 5 & -0.459213000 & 1.644756000 & -0.229903000 \\
\hline 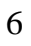 & 1.989434000 & 1.638330000 & 0.478710000 \\
\hline 5 & 0.214800000 & 2.530151000 & 1.984302000 \\
\hline 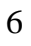 & 0.537223000 & 1.456486000 & 0.932031000 \\
\hline & 0.367103000 & 0.051008000 & 1.573794000 \\
\hline 7 & -0.675794000 & 1.194011000 & -2.543562000 \\
\hline 7 & 3.296058000 & 3.268802000 & -0.649655000 \\
\hline 7 & -1.611488000 & 3.179831000 & 3.363569000 \\
\hline 7 & 0.939959000 & -0.906575000 & 3.694422000 \\
\hline 8 & -1.747879000 & 1.729919000 & -2.407686000 \\
\hline & 0.119566000 & 1.062308000 & -1.397972000 \\
\hline 8 & -0.156366000 & 0.741227000 & -3.521314000 \\
\hline 8 & 4.152689000 & 2.423178000 & -0.595239000 \\
\hline & 2.059123000 & 2.961287000 & -0.068672000 \\
\hline 8 & 3.321834000 & 4.367121000 & -1.125367000 \\
\hline & -0.859767000 & 4.055822000 & 3.708666000 \\
\hline 0 & -1.130327000 & 2.269550000 & 2.408909000 \\
\hline & -0.016830000 & -1.626241000 & 3.531822000 \\
\hline 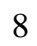 & 1.176648000 & 0.102117000 & 2.747776000 \\
\hline 8 & -2.733015000 & 2.940903000 & 3.704464000 \\
\hline 8 & 1.741431000 & -0.900565000 & 4.577229000 \\
\hline
\end{tabular}


BQ (ground state configuration):

Method: PBEO

$\begin{array}{lrrr}6 & -2.671067000 & -2.571243000 & -0.005945000 \\ 6 & -1.115788000 & -2.612664000 & -0.007369000 \\ 6 & -0.408032000 & -1.324031000 & -0.005768000 \\ 1 & 0.677391000 & -1.357365000 & -0.006770000 \\ 6 & -1.098520000 & -0.166426000 & -0.003175000 \\ 6 & -2.558107000 & -0.127569000 & -0.001839000 \\ 6 & -3.309205000 & -1.246788000 & -0.003112000 \\ 1 & -0.567987000 & 0.782382000 & -0.002005000 \\ 1 & -3.037410000 & 0.848119000 & 0.000257000 \\ 1 & -4.394861000 & -1.222285000 & -0.002126000 \\ 8 & -3.303442000 & -3.607519000 & -0.007179000 \\ 8 & -0.539413000 & -3.681189000 & -0.009708000\end{array}$




\begin{tabular}{lccc}
\multicolumn{4}{l}{ BQ-PETN (ground state configuration): } \\
Method: PBE0 & & \\
1 & 12.218925000 & 3.514166000 & 23.594493000 \\
1 & 12.959394000 & 5.020374000 & 21.054609000 \\
1 & 12.540006000 & 7.197190000 & 24.056572000 \\
1 & 9.769251000 & 6.114322000 & 22.576620000 \\
1 & 12.530382000 & 4.799724000 & 24.797665000 \\
1 & 11.410780000 & 5.849026000 & 20.741221000 \\
1 & 13.501820000 & 6.921315000 & 22.584824000 \\
1 & 9.973604000 & 4.529432000 & 23.364811000 \\
1 & 12.884461000 & 8.493412000 & 28.176164000 \\
1 & 11.561912000 & 8.286525000 & 30.258796000 \\
1 & 10.216713000 & 6.277104000 & 30.753554000 \\
1 & 10.102792000 & 4.365022000 & 29.183159000 \\
6 & 12.605240000 & 4.526658000 & 23.741345000 \\
6 & 11.918725000 & 5.117201000 & 21.375538000 \\
6 & 12.492602000 & 6.908330000 & 23.004325000 \\
6 & 10.375302000 & 5.542305000 & 23.284601000 \\
6 & 11.848807000 & 5.522960000 & 22.852824000 \\
6 & 11.435637000 & 5.275061000 & 27.706786000 \\
6 & 12.303849000 & 6.529699000 & 27.408820000 \\
6 & 12.289573000 & 7.611975000 & 28.394887000 \\
6 & 11.565532000 & 7.484539000 & 29.525218000 \\
6 & 10.769490000 & 6.295811000 & 29.818048000 \\
6 & 10.699307000 & 5.247628000 & 28.973910000 \\
7 & 14.832927000 & 3.737114000 & 24.006818000 \\
7 & 11.265914000 & 3.299418000 & 19.989233000 \\
7 & 12.042302000 & 9.152022000 & 22.357808000 \\
7 & 9.089529000 & 6.198379000 & 25.164203000 \\
8 & 13.975679000 & 4.588599000 & 23.309520000 \\
8 & 11.257974000 & 3.847344000 & 21.275935000 \\
8 & 11.649772000 & 7.815665000 & 22.274608000 \\
8 & 10.339279000 & 6.183579000 & 24.567864000 \\
8 & 15.975665000 & 3.842286000 & 23.656548000 \\
8 & 10.710796000 & 2.239461000 & 19.933691000 \\
8 & 11.307692000 & 9.888918000 & 21.761654000 \\
8 & 9.097942000 & 6.723598000 & 26.247129000 \\
8 & 14.341339000 & 3.019904000 & 24.840271000 \\
8 & 11.810101000 & 3.937739000 & 19.123372000 \\
8 & 13.036860000 & 9.396749000 & 22.993048000 \\
8 & 8.171557000 & 5.706352000 & 24.558317000 \\
8 & 11.393577000 & 4.373424000 & 26.891848000 \\
8 & 12.961408000 & 6.570347000 & 26.385195000
\end{tabular}


BQ-PETN $\left(\mathrm{T}_{1},{ }^{3}\left(\mathrm{n}, \pi^{*}\right)\right.$ state):

Method: PBE0, $\triangle S C F$

$\begin{array}{cccc}1 & 12.153010000 & 3.637932000 & 23.676981000 \\ 1 & 12.999146000 & 5.015231000 & 21.088391000 \\ 1 & 12.393677000 & 7.349766000 & 23.938643000 \\ 1 & 9.722659000 & 6.135717000 & 22.398393000 \\ 1 & 12.378968000 & 4.995875000 & 24.814034000 \\ 1 & 11.450017000 & 5.787957000 & 20.657812000 \\ 1 & 13.448491000 & 6.999057000 & 22.547762000 \\ 1 & 9.914037000 & 4.605149000 & 23.289044000 \\ 1 & 13.160442000 & 8.213093000 & 28.396222000 \\ 1 & 12.043955000 & 7.913452000 & 30.583632000 \\ 1 & 10.496624000 & 5.998804000 & 30.958919000 \\ 1 & 10.050171000 & 4.373128000 & 29.148242000 \\ 6 & 12.518056000 & 4.663358000 & 23.781490000 \\ 6 & 11.942973000 & 5.106358000 & 21.356505000 \\ 6 & 12.415565000 & 6.997036000 & 22.905207000 \\ 6 & 10.304043000 & 5.618258000 & 23.166940000 \\ 6 & 11.795877000 & 5.596533000 & 22.801869000 \\ 6 & 11.276109000 & 5.308254000 & 27.689456000 \\ 6 & 12.337179000 & 6.489325000 & 27.473299000 \\ 6 & 12.494748000 & 7.376463000 & 28.581252000 \\ 6 & 11.861083000 & 7.204787000 & 29.781994000 \\ 6 & 10.979101000 & 6.119154000 & 29.994014000 \\ 6 & 10.713756000 & 5.218219000 & 28.998681000 \\ 7 & 14.735457000 & 3.940262000 & 24.240094000 \\ 7 & 11.392111000 & 3.195213000 & 20.053854000 \\ 7 & 11.981418000 & 9.191928000 & 22.102504000 \\ 7 & 8.938675000 & 6.305165000 & 24.982277000 \\ 8 & 13.908734000 & 4.723577000 & 23.430760000 \\ 8 & 11.314133000 & 3.817951000 & 21.304435000 \\ 8 & 11.609866000 & 7.846042000 & 22.072964000 \\ 8 & 10.203689000 & 6.334599000 & 24.404292000 \\ 8 & 15.894308000 & 4.037180000 & 23.948353000 \\ 8 & 10.863580000 & 2.120648000 & 20.038174000 \\ 8 & 11.281191000 & 9.880896000 & 21.415713000 \\ 8 & 8.888973000 & 6.906713000 & 26.019553000 \\ 8 & 14.203656000 & 3.282334000 & 25.097589000 \\ 8 & 11.960827000 & 3.794833000 & 19.176326000 \\ 8 & 12.928052000 & 9.487307000 & 22.787540000 \\ 8 & 8.072585000 & 5.705681000 & 24.395816000 \\ 8 & 11.040913000 & 4.556978000 & 26.751200000 \\ 8 & 12.913271000 & 6.573494000 & 26.395621000 \\ & & & \end{array}$


TS structure of hydrogen abstraction reaction in BQ-PETN $\left(T_{1},{ }^{3}\left(n, \pi^{*}\right)\right.$ state) complex: Method: PBE0, $\triangle$ SCF

$\begin{array}{cccc}1 & 12.279801000 & 4.040064000 & 24.260091000 \\ 1 & 12.960505000 & 5.001692000 & 21.339194000 \\ 1 & 12.351305000 & 7.757787000 & 23.780775000 \\ 1 & 9.692692000 & 6.134136000 & 22.568637000 \\ 1 & 12.551610000 & 5.670723000 & 25.155052000 \\ 1 & 11.348756000 & 5.610107000 & 20.875228000 \\ 1 & 13.394945000 & 7.193822000 & 22.451343000 \\ 1 & 9.996546000 & 4.863851000 & 23.787409000 \\ 1 & 13.425282000 & 7.564160000 & 28.418648000 \\ 1 & 12.184948000 & 7.083244000 & 30.507087000 \\ 1 & 10.347296000 & 5.407212000 & 30.541587000 \\ 1 & 9.726792000 & 4.197287000 & 28.456374000 \\ 6 & 12.613243000 & 5.056065000 & 24.051345000 \\ 6 & 11.913421000 & 5.078704000 & 21.646030000 \\ 6 & 12.369440000 & 7.222156000 & 22.829261000 \\ 6 & 10.320112000 & 5.836806000 & 23.413782000 \\ 6 & 11.798552000 & 5.805249000 & 22.997943000 \\ 6 & 11.200111000 & 5.168483000 & 27.225946000 \\ 6 & 12.306155000 & 6.168236000 & 27.235868000 \\ 6 & 12.622452000 & 6.835172000 & 28.437361000 \\ 6 & 11.924379000 & 6.558456000 & 29.592766000 \\ 6 & 10.874982000 & 5.600865000 & 29.612716000 \\ 6 & 10.526567000 & 4.930425000 & 28.475143000 \\ 7 & 14.826312000 & 4.239076000 & 24.407737000 \\ 7 & 11.381855000 & 2.967626000 & 20.697800000 \\ 7 & 11.836863000 & 9.215463000 & 21.651936000 \\ 7 & 8.979685000 & 6.820815000 & 25.098993000 \\ 8 & 13.961294000 & 5.130063000 & 23.691215000 \\ 8 & 11.362843000 & 3.771382000 & 21.846472000 \\ 8 & 11.516308000 & 7.871912000 & 21.877993000 \\ 8 & 10.214490000 & 6.814723000 & 24.452729000 \\ 8 & 15.966323000 & 4.378584000 & 24.083139000 \\ 8 & 10.915360000 & 1.881842000 & 20.885185000 \\ 8 & 11.096981000 & 9.741282000 & 20.870742000 \\ 8 & 8.950138000 & 7.575516000 & 26.030878000 \\ 8 & 14.310433000 & 3.502936000 & 25.199624000 \\ 8 & 11.851182000 & 3.452656000 & 19.698897000 \\ 8 & 12.785721000 & 9.660660000 & 22.246242000 \\ 8 & 8.118984000 & 6.103212000 & 24.656675000 \\ 8 & 10.894294000 & 4.591331000 & 26.163160000 \\ 8 & 12.970211000 & 6.424730000 & 26.158331000 \\ & & & \end{array}$




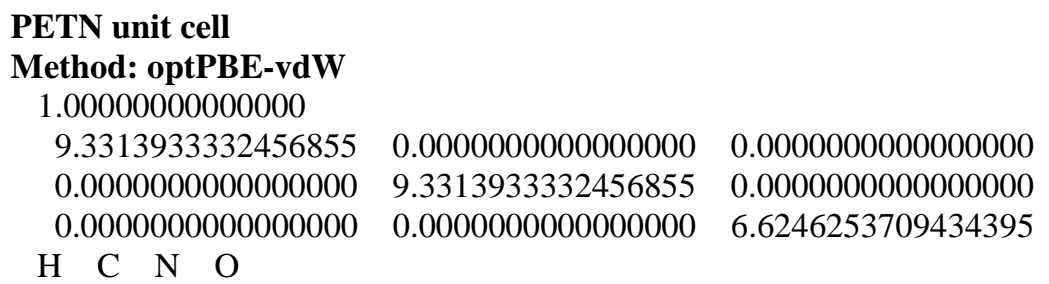


$\begin{array}{llll}0.8241237605727921 & 0.6028367153718225 & -0.0088592298786111\end{array}$ $\begin{array}{llll}0.6028367153718225 & 0.1758762094272057 & 0.0088591998786158\end{array}$ $\begin{array}{llll}0.3971632546281753 & 0.8241237605727921 & 0.0088591998786158\end{array}$ $\begin{array}{llll}0.3603575258390803 & 0.0936905966330703 & 0.3280072862127559\end{array}$ $\begin{array}{llll}0.6396424741609198 & 0.9063094253669313 & 0.3280072862127559\end{array}$ $\begin{array}{llll}0.0936905966330703 & 0.6396424741609198 & 0.6719927137872443\end{array}$ $\begin{array}{llll}0.9063094253669313 & 0.3603575258390803 & 0.6719927137872443\end{array}$ $\begin{array}{llll}0.1396424741609194 & 0.5936905746330687 & 0.1719927137872442\end{array}$ $\begin{array}{llll}0.8603575258390802 & 0.4063093953669291 & 0.1719927137872442\end{array}$ $\begin{array}{lllll}0.4063093953669291 & 0.1396424741609194 & 0.8280072862127557\end{array}$ $\begin{array}{llll}0.5936905746330687 & 0.8603575258390802 & 0.8280072862127557\end{array}$

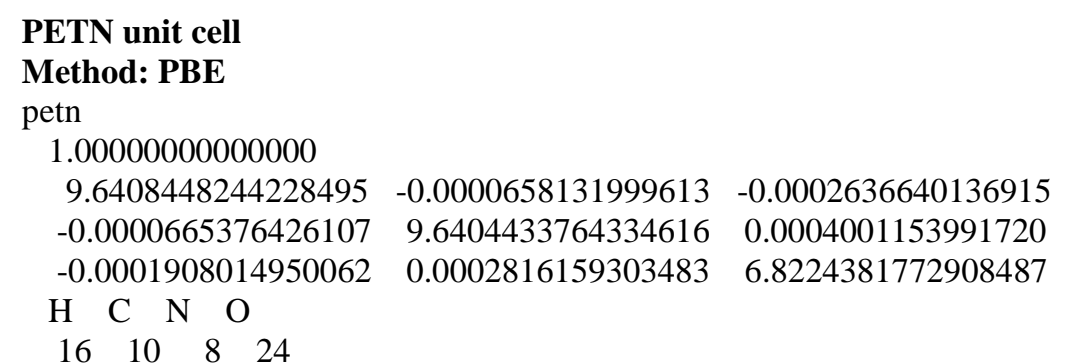

Direct

$\begin{array}{llll}0.6513099207548380 & 0.5857981341202338 & 0.7058242651856360\end{array}$ $\begin{array}{llll}0.7086920712936391 & 0.4143166523324799 & 0.4673482819266918\end{array}$ $\begin{array}{llll}0.3486775148422141 & 0.4142232774471092 & 0.7058263464310014\end{array}$ $\begin{array}{llll}0.2913006799302287 & 0.5856635486943984 & 0.4672977876250705\end{array}$ $\begin{array}{llll}0.4142052551967064 & 0.6513264949143127 & 0.2941625149857136\end{array}$ $\begin{array}{lllll}0.5856673848482626 & 0.7086969873757827 & 0.5326621920533604\end{array}$ $\begin{array}{llll}0.5857888571932246 & 0.3486840487662887 & 0.2941902089242219\end{array}$ $\begin{array}{llll}0.4143052747386862 & 0.2913114694108942 & 0.5326711237974422\end{array}$ $\begin{array}{llll}0.8486792729431670 & 0.0857992175693608 & 0.7941616511867943\end{array}$ $\begin{array}{llll}0.7913086034888606 & 0.9143514329170331 & 0.0326848726156446\end{array}$ $\begin{array}{llll}0.1513092736556374 & 0.9142154040852478 & 0.7941868533398727\end{array}$ $\begin{array}{llll}0.2086927043568987 & 0.0856914520831697 & 0.0326785545583786\end{array}$ $\begin{array}{llll}0.0857837048407425 & 0.1513230740220129 & 0.2058315685413773\end{array}$ $\begin{array}{llll}0.9143383467542108 & 0.2087024801777871 & 0.9672996213259920\end{array}$ $\begin{array}{llll}0.9142041616508533 & 0.8486904291306387 & 0.2058263173701190\end{array}$ $\begin{array}{llll}0.0856788737212592 & 0.7913110302736955 & 0.9673466547484191\end{array}$ $\begin{array}{llll}0.3803174445413207 & 0.5586477047358032 & 0.3746261418199998\end{array}$ $\begin{array}{llll}0.6196899496758100 & 0.4413474653614768 & 0.3746371549328337\end{array}$ $\begin{array}{llll}0.5586397792976427 & 0.6196833528664610 & 0.6253598398143622\end{array}$ $\begin{array}{llll}0.4999974711728961 & 0.4999983914338910 & 0.4999976178480015\end{array}$ $\begin{array}{llll}0.4413390757606706 & 0.3803109833360466 & 0.6253689373661788\end{array}$ $\begin{array}{llll}0.9413536121544616 & 0.1196838570313806 & 0.8746273592565228\end{array}$ $\begin{array}{llll}0.0586480232610390 & 0.8803185605292342 & 0.8746419174612294\end{array}$ $\begin{array}{llll}0.1196869366058367 & 0.0586609316964354 & 0.1253654852429934\end{array}$ $0.00000290866208520 .0000076284471241-0.0000016459861327$ $\begin{array}{llll}0.8803323930301249 & 0.9413711934179503 & 0.1253665413941025\end{array}$ $\begin{array}{llll}0.2148401986748854 & 0.4848945201121554 & 0.1288199891198163\end{array}$ $\begin{array}{llll}0.7851818242784091 & 0.5150923605087122 & 0.1287033527194902\end{array}$ $\begin{array}{llll}0.4849076262958401 & 0.7851538304053745 & 0.8712071261581480\end{array}$ $\begin{array}{llll}0.5150823431660752 & 0.2148193374208349 & 0.8713047011024245\end{array}$ $\begin{array}{llll}0.2851585634514213 & 0.9848639723741158 & 0.3712447267915319\end{array}$ $\begin{array}{llll}0.7148424387297755 & 0.0151205842950142 & 0.3711379899050057\end{array}$ $\begin{array}{llll}0.0150978168976070 & 0.2851495730810734 & 0.6288110530656857\end{array}$ $0.98491231691766950 .7148520819347555 \quad 0.6287606049257176$ 
$\begin{array}{llll}0.1531070420527642 & 0.5899352820018980 & 0.1748950005807003\end{array}$ $\begin{array}{lllll}0.3409224360156939 & 0.4520075309937885 & 0.2355054595614744\end{array}$ $\begin{array}{lllll}0.1890017751668705 & 0.3983831286140602 & 0.0039575731473560\end{array}$ $\begin{array}{lllll}0.8468875479842456 & 0.4099858902916623 & 0.1745712446206795\end{array}$ $\begin{array}{lllll}0.6591106413355525 & 0.5479921421597440 & 0.2355128960314999\end{array}$ $\begin{array}{lllll}0.8109949371953861 & 0.6016708031362541 & 0.0039546413901425\end{array}$ $\begin{array}{llll}0.5899703326245250 & 0.8468946049813673 & 0.8251938082708287\end{array}$ $\begin{array}{lllll}0.4520016650684118 & 0.6590944908271941 & 0.7644727041058388\end{array}$ $\begin{array}{lllll}0.3983767068924835 & 0.8109927789140904 & 0.9960439983650502\end{array}$ $\begin{array}{lllll}0.4099812976129615 & 0.1531113169084999 & 0.8254367432458061\end{array}$ $\begin{array}{lllll}0.5479835795610073 & 0.3408908168968345 & 0.7644871593104090\end{array}$ $\begin{array}{lllll}0.6016614484639755 & 0.1890013100257519 & 0.9960449466510565\end{array}$ $\begin{array}{lllll}0.3468608650831286 & 0.0899558857933803 & 0.3253809377345827\end{array}$ $\begin{array}{lllll}0.1590862023862846 & 0.9519994236894016 & 0.2644850532360380\end{array}$ $\begin{array}{lllll}0.3110089515507467 & 0.8982745385289604 & 0.4959607460272659\end{array}$ $\begin{array}{lllll}0.9100675739467022 & 0.3468921067445823 & 0.6749058604712697\end{array}$ $\begin{array}{llll}0.6530949327535873 & 0.9100839649358043 & 0.3250577257485279\end{array}$ $\begin{array}{lllll}0.8409321248813522 & 0.0480023907051798 & 0.2644932484216030\end{array}$ $\begin{array}{lllll}0.6890038545601133 & 0.1016154179675515 & 0.4960243732602648\end{array}$ $\begin{array}{lllll}0.0479917027809153 & 0.1590712865703323 & 0.7355048801409677\end{array}$ $\begin{array}{llll}0.1016116249747604 & 0.3109994712703901 & 0.5039576922412349\end{array}$ $\begin{array}{llll}0.0900099089554726 & 0.6531490402309653 & 0.6746860118089827\end{array}$ $\begin{array}{lllll}0.9520000406682767 & 0.8409061326210638 & 0.7355262254606295\end{array}$ $\begin{array}{lllll}0.8983601847017701 & 0.6890087828829371 & 0.5039733626141255\end{array}$

\title{
PETN (110) surface
}

Method: optPBE-vdW

\author{
CIF file \\ 1. \\ $\begin{array}{llll}14.7659997940000007 & 0.0000000000000000 & 0.000000000000000\end{array}$ \\ $\begin{array}{llll}8.8218669359999993 & 11.8410056025999992 & 0.0000000000000000\end{array}$ \\ $\begin{array}{llll}0.0000000000000000 & 0.0000000000000000 & 45.7868003844999976\end{array}$ \\ $\begin{array}{llll}\mathrm{H} & \mathrm{C} & \mathrm{N} & \mathrm{O}\end{array}$ \\ $\begin{array}{llll}128 & 80 & 64 & 192\end{array}$
}

Direct

$\begin{array}{lllll}0.3372333211277863 & 0.1253064307496944 & 0.1002156867725026\end{array}$ $\begin{array}{lllll}0.8372333211277864 & 0.6253064307496941 & 0.1002156867725026\end{array}$ $\begin{array}{llll}0.0870859778001570 & 0.8757895737454053 & 0.2447384069141983\end{array}$ $\begin{array}{lllll}0.5870859778001566 & 0.3757895737454054 & 0.2447384069141983\end{array}$ $\begin{array}{lllll}0.3372261384373652 & 0.1257433120682827 & 0.3888723059340504\end{array}$ $\begin{array}{lllll}0.8372261384373652 & 0.6257433120682834 & 0.3888723059340504\end{array}$ $\begin{array}{lllll}0.0854696943391812 & 0.8782480453083319 & 0.5339685613140455\end{array}$ $\begin{array}{lllll}0.5854696943391812 & 0.3782480453083323 & 0.5339685613140455\end{array}$ $\begin{array}{llll}0.3712174294016952 & 0.1648361084310973 & 0.0292121458303746\end{array}$ $\begin{array}{lllll}0.8712174294016953 & 0.6648361084310972 & 0.0292121458303746\end{array}$ $\begin{array}{lllll}0.1243503598797060 & 0.9124924143836377 & 0.1742701457454989\end{array}$ $\begin{array}{llll}0.6243503598797061 & 0.4124924143836383 & 0.1742701457454989\end{array}$ $\begin{array}{lllll}0.3744152103130630 & 0.1627105601771698 & 0.3184095539440464\end{array}$ $\begin{array}{lllll}0.8744152103130630 & 0.6627105601771697 & 0.3184095539440464\end{array}$ $\begin{array}{lllll}0.1246021535368929 & 0.9129009304941648 & 0.4628606372354099\end{array}$ $\begin{array}{lllll}0.6246021535368931 & 0.4129009304941651 & 0.4628606372354099\end{array}$ $\begin{array}{lllll}0.2026209678822125 & 0.4178088066637231 & 0.0758086667111123\end{array}$ $\begin{array}{lllll}0.7026209538822111 & 0.9178087766637205 & 0.0758086667111123\end{array}$ $\begin{array}{lllll}0.9553649131990466 & 0.1666286007969887 & 0.2201507352952281\end{array}$ $\begin{array}{lllll}0.4553649421990447 & 0.6666285857969912 & 0.2201507352952281\end{array}$ 
$\begin{array}{llll}0.2054220925219253 & 0.4167685403705773 & 0.3643106254139783\end{array}$ $\begin{array}{llll}0.7054220785219244 & 0.9167685103705747 & 0.3643106254139783\end{array}$ $\begin{array}{llll}0.9552911214159134 & 0.1675526802119716 & 0.5082368802937095\end{array}$ $\begin{array}{llll}0.4552911504159121 & 0.6675526652119741 & 0.5082368802937095\end{array}$ $\begin{array}{llll}0.0820126138125060 & 0.2950084045123762 & 0.0550361989503978\end{array}$ $\begin{array}{lllll}0.5820126358125077 & 0.7950084335123750 & 0.0550361989503978\end{array}$ $\begin{array}{llll}0.8331579459103188 & 0.0443669260773149 & 0.1988167920255177\end{array}$ $\begin{array}{llll}0.3331579159103166 & 0.5443669440773162 & 0.1988167920255177\end{array}$ $\begin{array}{llll}0.0831920730666373 & 0.2945890033092527 & 0.3429799440713545\end{array}$ $\begin{array}{llll}0.5831920950666390 & 0.7945890323092519 & 0.3429799440713545\end{array}$ $\begin{array}{llll}0.8324345195238417 & 0.0471308182525321 & 0.4873850389509491\end{array}$ $\begin{array}{llll}0.3324344895238390 & 0.5471308362525333 & 0.4873850389509491\end{array}$ $\begin{array}{llll}0.0821912013362776 & 0.7973790461177889 & 0.0758086667111123\end{array}$ $\begin{array}{llll}0.5821912233362795 & 0.2973790171177896 & 0.0758086667111123\end{array}$ $\begin{array}{lllll}0.8333714142030088 & 0.5446350868009534 & 0.2201507352952281\end{array}$ $\begin{array}{llll}0.3333713842030064 & 0.0446350868009538 & 0.2201507352952281\end{array}$ $\begin{array}{llll}0.0832314676294233 & 0.7945779214780756 & 0.3643106254139783\end{array}$ $\begin{array}{llll}0.5832314896294253 & 0.2945778924780770 & 0.3643106254139783\end{array}$ $\begin{array}{llll}0.8324473347880259 & 0.5447088785840866 & 0.5082368802937095\end{array}$ $\begin{array}{lllll}0.3324473047880238 & 0.0447088785840867 & 0.5082368802937095\end{array}$ $\begin{array}{llll}0.2049915804876262 & 0.9179873641874923 & 0.0550361989503978\end{array}$ $\begin{array}{llll}0.7049915664876250 & 0.4179873941874945 & 0.0550361989503978\end{array}$ $\begin{array}{llll}0.9556330559226838 & 0.6668420540896812 & 0.1988167920255177\end{array}$ $\begin{array}{lllll}0.4556330849226826 & 0.1668421140896788 & 0.1988167920255177\end{array}$ $\begin{array}{llll}0.2054109816907495 & 0.9168079049333610 & 0.3429799440713545\end{array}$ $\begin{array}{llll}0.7054109676907481 & 0.4168079349333634 & 0.3429799440713545\end{array}$ $\begin{array}{llll}0.9528691637474667 & 0.6675654804761583 & 0.4873850389509491\end{array}$ $\begin{array}{llll}0.4528691927474653 & 0.1675655404761563 & 0.4873850389509491\end{array}$ $\begin{array}{llll}0.3351638915689027 & 0.6287825705983047 & 0.0292121458303746\end{array}$ $\begin{array}{llll}0.8351638915689028 & 0.1287825705983048 & 0.0292121458303746\end{array}$ $\begin{array}{llll}0.0875075856163619 & 0.3756496401202941 & 0.1742701457454989\end{array}$ $\begin{array}{llll}0.5875075856163623 & 0.8756496401202939 & 0.1742701457454989\end{array}$ $\begin{array}{llll}0.3372894398228302 & 0.6255847896869370 & 0.3184095539440464\end{array}$ $\begin{array}{llll}0.8372894398228303 & 0.1255847896869369 & 0.3184095539440464\end{array}$ $\begin{array}{llll}0.0870990695058351 & 0.3753978464631070 & 0.4628606372354099\end{array}$ $\begin{array}{llll}0.5870990695058352 & 0.8753978464631069 & 0.4628606372354099\end{array}$ $\begin{array}{lllll}0.3746935692503054 & 0.6627666788722136 & 0.1002156867725026\end{array}$ $\begin{array}{llll}0.8746935692503059 & 0.1627666788722137 & 0.1002156867725026\end{array}$ $\begin{array}{llll}0.1242104262545947 & 0.4129140221998430 & 0.2447384069141983\end{array}$ $\begin{array}{lllll}0.6242104262545947 & 0.9129140221998434 & 0.2447384069141983\end{array}$ $\begin{array}{llll}0.3742566879317174 & 0.6627738615626348 & 0.3888723059340504\end{array}$ $\begin{array}{lllll}0.8742566879317166 & 0.1627738615626346 & 0.3888723059340504\end{array}$ $\begin{array}{llll}0.1217519546916678 & 0.4145303056608186 & 0.5339685613140455\end{array}$ $\begin{array}{llll}0.6217519546916681 & 0.9145303056608188 & 0.5339685613140455\end{array}$ $\begin{array}{llll}0.2991512147244037 & 0.2620879027719115 & 0.1091109015329706\end{array}$ $\begin{array}{llll}0.7991511847244009 & 0.7620879327719063 & 0.1091109015329706\end{array}$ $\begin{array}{llll}0.0491536915352976 & 0.0126986645586394 & 0.2534567233843933\end{array}$ $\begin{array}{llll}0.5491536655352952 & 0.5126986935586382 & 0.2534567233843933\end{array}$ $\begin{array}{llll}0.2996793594713839 & 0.2624970246436032 & 0.3976129383517948\end{array}$ $\begin{array}{llll}0.7996793294713820 & 0.7624970546435987 & 0.3976129383517948\end{array}$ $\begin{array}{llll}0.0489715672500207 & 0.0152292814570962 & 0.5419666503244811\end{array}$ $\begin{array}{llll}0.5489715412500183 & 0.5152293104570951 & 0.5419666503244811\end{array}$ $\begin{array}{llll}0.2341773993229109 & 0.2013288388296829 & 0.0212539575636160\end{array}$ $\begin{array}{llll}0.7341773693229158 & 0.7013288688296854 & 0.0212539575636160\end{array}$ $\begin{array}{llll}0.9876555498343711 & 0.9499138977088711 & 0.1655047870612625\end{array}$ $\begin{array}{llll}0.4876555798343666 & 0.4499138677088684 & 0.1655047870612625\end{array}$ 
$\begin{array}{llll}0.2376623755087299 & 0.2002820039673040 & 0.3096368760809975\end{array}$ $\begin{array}{llll}0.7376623455087344 & 0.7002820339673069 & 0.3096368760809975\end{array}$ $\begin{array}{llll}0.9878971205862682 & 0.9504973962123192 & 0.4541183134067271\end{array}$ $\begin{array}{llll}0.4878971505862638 & 0.4504973662123169 & 0.4541183134067271\end{array}$ $\begin{array}{llll}0.3081617962188624 & 0.3450926213567670 & 0.0480396805475487\end{array}$ $\begin{array}{llll}0.8081618262188647 & 0.8450926513567623 & 0.0480396805475487\end{array}$ $\begin{array}{llll}0.0577070210125911 & 0.0946919940867649 & 0.1915722961764662\end{array}$ $\begin{array}{llll}0.5577070400125965 & 0.5946920170867630 & 0.1915722961764662\end{array}$ $\begin{array}{llll}0.3077641413808943 & 0.3448668288915588 & 0.3357193565155949\end{array}$ $\begin{array}{llll}0.8077641713808968 & 0.8448668588915546 & 0.3357193565155949\end{array}$ $\begin{array}{llll}0.0581208230386706 & 0.0943043844508355 & 0.4798082916205920\end{array}$ $\begin{array}{llll}0.5581208420386755 & 0.5943044074508339 & 0.4798082916205920\end{array}$ $\begin{array}{llll}0.1553775543174508 & 0.1922467211605288 & 0.0834729858218414\end{array}$ $\begin{array}{llll}0.6553775243174554 & 0.6922467061605243 & 0.0834729858218414\end{array}$ $\begin{array}{llll}0.9052804096677034 & 0.9419888364126096 & 0.2273937817812780\end{array}$ $\begin{array}{llll}0.4052804396676986 & 0.4419888664126119 & 0.2273937817812780\end{array}$ $\begin{array}{llll}0.1553125290109317 & 0.1921199310404150 & 0.3715361344891194\end{array}$ $\begin{array}{llll}0.6553124990109359 & 0.6921199160404102 & 0.3715361344891194\end{array}$ $\begin{array}{llll}0.9050817541508815 & 0.9416053147012051 & 0.5151723853073770\end{array}$ $\begin{array}{llll}0.4050817841508774 & 0.4416053447012075 & 0.5151723853073770\end{array}$ $\begin{array}{llll}0.1549073786432331 & 0.6918381737811353 & 0.0480396805475487\end{array}$ $\begin{array}{lllll}0.6549073486432377 & 0.1918381887811398 & 0.0480396805475487\end{array}$ $\begin{array}{llll}0.9053079829132370 & 0.4422929899874062 & 0.1915722961764662\end{array}$ $\begin{array}{lllll}0.4053080129132321 & 0.9422929599874035 & 0.1915722961764662\end{array}$ $\begin{array}{llll}0.1551331711084411 & 0.6922358286191032 & 0.3357193565155949\end{array}$ $\begin{array}{llll}0.6551331411084454 & 0.1922358436191078 & 0.3357193565155949\end{array}$ $\begin{array}{llll}0.9056955925491661 & 0.4418791879613269 & 0.4798082916205920\end{array}$ $\begin{array}{llll}0.4056956225491614 & 0.9418791579613245 & 0.4798082916205920\end{array}$ $\begin{array}{llll}0.3077532638394736 & 0.8446224756825446 & 0.0834729858218414\end{array}$ $\begin{array}{lllll}0.8077532938394757 & 0.3446224456825492 & 0.0834729858218414\end{array}$ $\begin{array}{llll}0.0580111445873855 & 0.5947195903322966 & 0.2273937817812780\end{array}$ $\begin{array}{lllll}0.5580111635873904 & 0.0947195903322968 & 0.2273937817812780\end{array}$ $\begin{array}{llll}0.3078800539595873 & 0.8446875009890641 & 0.3715361344891194\end{array}$ $\begin{array}{llll}0.8078800839595898 & 0.3446874709890683 & 0.3715361344891194\end{array}$ $\begin{array}{llll}0.0583946662987898 & 0.5949182458491185 & 0.5151723853073770\end{array}$ $\begin{array}{llll}0.5583946852987949 & 0.0949182458491181 & 0.5151723853073770\end{array}$ $\begin{array}{lllll}0.2986711611703173 & 0.7658226306770842 & 0.0212539575636160\end{array}$ $\begin{array}{llll}0.7986711311703146 & 0.2658226006770889 & 0.0212539575636160\end{array}$ $\begin{array}{llll}0.0500861282911312 & 0.5123444501656289 & 0.1655047870612625\end{array}$ $\begin{array}{llll}0.5500861022911289 & 0.0123443901656310 & 0.1655047870612625\end{array}$ $\begin{array}{llll}0.2997179960326958 & 0.7623376544912656 & 0.3096368760809975\end{array}$ $\begin{array}{llll}0.7997179660326931 & 0.2623376244912700 & 0.3096368760809975\end{array}$ $\begin{array}{llll}0.0495026297876829 & 0.5121028794137318 & 0.4541183134067271\end{array}$ $\begin{array}{llll}0.5495026037876808 & 0.0121028194137339 & 0.4541183134067271\end{array}$ $\begin{array}{llll}0.2379120972280888 & 0.7008488152755991 & 0.1091109015329706\end{array}$ $\begin{array}{llll}0.7379120672280937 & 0.2008487852755965 & 0.1091109015329706\end{array}$ $\begin{array}{llll}0.9873013064413618 & 0.4508463044647019 & 0.2534567233843933\end{array}$ $\begin{array}{llll}0.4873013364413572 & 0.9508463344647048 & 0.2534567233843933\end{array}$ $\begin{array}{llll}0.2375029753563969 & 0.7003206705286180 & 0.3976129383517948\end{array}$ $\begin{array}{llll}0.7375029453564013 & 0.2003206405286160 & 0.3976129383517948\end{array}$ $\begin{array}{llll}0.9847706895429049 & 0.4510284287499789 & 0.5419666503244811\end{array}$ $\begin{array}{lllll}0.4847707195429004 & 0.9510284587499817 & 0.5419666503244811\end{array}$ $\begin{array}{llll}0.3294385036095309 & 0.1991244528948646 & 0.0917645919552202\end{array}$ $\begin{array}{llll}0.8294385036095310 & 0.6991244228948621 & 0.0917645919552202\end{array}$ $\begin{array}{llll}0.0793367346819308 & 0.9495055013982552 & 0.2361941123002787\end{array}$ $\begin{array}{llll}0.5793367346819309 & 0.4495055313982577 & 0.2361941123002787\end{array}$ 
$\begin{array}{llll}0.3295812428645530 & 0.1994143825518317 & 0.3803328236815231\end{array}$ $\begin{array}{llll}0.8295812428645535 & 0.6994143525518294 & 0.3803328236815231\end{array}$ $\begin{array}{llll}0.0790291296000967 & 0.9507533482883821 & 0.5249748666721010\end{array}$ $\begin{array}{llll}0.5790291296000968 & 0.4507533782883845 & 0.5249748666721010\end{array}$ $\begin{array}{llll}0.2987416162350623 & 0.1712564091352964 & 0.0382219413165277\end{array}$ $\begin{array}{llll}0.7987416462350649 & 0.6712564091352961 & 0.0382219413165277\end{array}$ $\begin{array}{llll}0.0506592349070444 & 0.9201456812558023 & 0.1827939626604686\end{array}$ $\begin{array}{llll}0.5506592609070465 & 0.4201456812558023 & 0.1827939626604686\end{array}$ $\begin{array}{llll}0.3007064047915038 & 0.1703951563663780 & 0.3269263536150623\end{array}$ $\begin{array}{llll}0.8007064347915066 & 0.6703951563663779 & 0.3269263536150623\end{array}$ $\begin{array}{llll}0.0510599507874270 & 0.9203157543359256 & 0.4713952427964285\end{array}$ $\begin{array}{llll}0.5510599767874291 & 0.4203157543359257 & 0.4713952427964285\end{array}$ $\begin{array}{llll}0.2307027622560807 & 0.3615833962108371 & 0.0568684309202650\end{array}$ $\begin{array}{llll}0.7307027322560850 & 0.8615833962108375 & 0.0568684309202650\end{array}$ $\begin{array}{llll}0.9812390727313524 & 0.1111580981041969 & 0.2011176663891436\end{array}$ $\begin{array}{llll}0.4812391027313475 & 0.6111580981041969 & 0.2011176663891436\end{array}$ $\begin{array}{llll}0.2313340707244611 & 0.3612963621793128 & 0.3452800306443858\end{array}$ $\begin{array}{llll}0.7313340407244661 & 0.8612963621793125 & 0.3452800306443858\end{array}$ $\begin{array}{llll}0.9814936433516249 & 0.1112236664518192 & 0.4893865502757879\end{array}$ $\begin{array}{llll}0.4814936733516202 & 0.6112236664518190 & 0.4893865502757879\end{array}$ $\begin{array}{llll}0.1384015981661009 & 0.2688585549817373 & 0.0738762025225538\end{array}$ $\begin{array}{llll}0.6384015981661013 & 0.7688585849817330 & 0.0738762025225538\end{array}$ $\begin{array}{llll}0.8887396508248411 & 0.0184510706620589 & 0.2178209950916891\end{array}$ $\begin{array}{llll}0.3887396508248413 & 0.5184510986620540 & 0.2178209950916891\end{array}$ $\begin{array}{llll}0.1387944518240933 & 0.2686157987537844 & 0.3619867067597446\end{array}$ $\begin{array}{lllll}0.6387944518240933 & 0.7686158287537794 & 0.3619867067597446\end{array}$ $\begin{array}{llll}0.8886771981572217 & 0.0189956830832043 & 0.5063215237259465\end{array}$ $\begin{array}{llll}0.3886771981572216 & 0.5189957110832001 & 0.5063215237259465\end{array}$ $\begin{array}{llll}0.1384166037891629 & 0.7692972677439150 & 0.0568684309202650\end{array}$ $\begin{array}{llll}0.6384166037891625 & 0.2692972377439194 & 0.0568684309202650\end{array}$ $\begin{array}{llll}0.8888419018958031 & 0.5187609272686476 & 0.2011176663891436\end{array}$ $\begin{array}{llll}0.3888419018958032 & 0.0187609272686479 & 0.2011176663891436\end{array}$ $\begin{array}{llll}0.1387036378206873 & 0.7686659592755339 & 0.3452800306443858\end{array}$ $\begin{array}{llll}0.6387036378206875 & 0.2686659292755388 & 0.3452800306443858\end{array}$ $\begin{array}{lllll}0.8887763335481810 & 0.5185063566483751 & 0.4893865502757879\end{array}$ $\begin{array}{lllll}0.3887763335481809 & 0.0185063566483751 & 0.4893865502757879\end{array}$ $\begin{array}{lllll}0.2311414450182627 & 0.8615984018338987 & 0.0738762025225538\end{array}$ $\begin{array}{llll}0.7311414150182670 & 0.3615984018338993 & 0.0738762025225538\end{array}$ $\begin{array}{llll}0.9815489013379460 & 0.6112603491751589 & 0.2178209950916891\end{array}$ $\begin{array}{lllll}0.4815489313379414 & 0.1112604091751564 & 0.2178209950916891\end{array}$ $\begin{array}{lllll}0.2313842012462156 & 0.8612055481759067 & 0.3619867067597446\end{array}$ $\begin{array}{lllll}0.7313841712462206 & 0.3612055481759067 & 0.3619867067597446\end{array}$ $\begin{array}{llll}0.9810042889167999 & 0.6113228018427783 & 0.5063215237259465\end{array}$ $\begin{array}{llll}0.4810043189167959 & 0.1113228618427763 & 0.5063215237259465\end{array}$ $\begin{array}{lllll}0.3287435908647036 & 0.7012583537649351 & 0.0382219413165277\end{array}$ $\begin{array}{llll}0.8287435908647039 & 0.2012583837649376 & 0.0382219413165277\end{array}$ $\begin{array}{llll}0.0798543187441976 & 0.4493407690929558 & 0.1827939626604686\end{array}$ $\begin{array}{llll}0.5798543187441977 & 0.9493407390929535 & 0.1827939626604686\end{array}$ $\begin{array}{lllll}0.3296048436336219 & 0.6992935652084934 & 0.3269263536150623\end{array}$ $\begin{array}{llll}0.8296048436336221 & 0.1992935952084962 & 0.3269263536150623\end{array}$ $\begin{array}{llll}0.0796842456640744 & 0.4489400532125733 & 0.4713952427964285\end{array}$ $\begin{array}{llll}0.5796842456640744 & 0.9489400232125709 & 0.4713952427964285\end{array}$ $\begin{array}{llll}0.3008755471051355 & 0.6705614963904690 & 0.0917645919552202\end{array}$ $\begin{array}{llll}0.8008755771051379 & 0.1705614963904689 & 0.0917645919552202\end{array}$ $\begin{array}{llll}0.0504944726017427 & 0.4206632653180691 & 0.2361941123002787\end{array}$ $\begin{array}{llll}0.5504944986017448 & 0.9206632653180691 & 0.2361941123002787\end{array}$ 
$\begin{array}{llll}0.3005856174481683 & 0.6704187571354465 & 0.3803328236815231\end{array}$ $\begin{array}{llll}0.8005856474481706 & 0.1704187571354470 & 0.3803328236815231\end{array}$ $\begin{array}{llll}0.0492466257116159 & 0.4209708703999032 & 0.5249748666721010\end{array}$ $\begin{array}{llll}0.5492466517116179 & 0.9209708703999032 & 0.5249748666721010\end{array}$ $\begin{array}{lllll}0.2494862270110113 & 0.2501753196023228 & 0.0651369668704198\end{array}$ $\begin{array}{llll}0.7494862270110114 & 0.7501753196023225 & 0.0651369668704198\end{array}$ $\begin{array}{llll}-0.0000292358778634 & -0.0001645500416413 & 0.2094545286181233\end{array}$ $\begin{array}{lllll}0.4999707641221366 & 0.4998354499583587 & 0.2094545286181233\end{array}$ $\begin{array}{llll}0.2501216493690404 & 0.2499040147287957 & 0.3536229838618800\end{array}$ $\begin{array}{llll}0.7501216493690401 & 0.7499040147287962 & 0.3536229838618800\end{array}$ $\begin{array}{llll}0.0001605816384242 & 0.0001371949892787 & 0.4980574774096541\end{array}$ $\begin{array}{llll}0.5001605816384239 & 0.5001371949892786 & 0.4980574774096541\end{array}$ $\begin{array}{llll}0.2498246803976773 & 0.7505137729889886 & 0.0651369668704198\end{array}$ $\begin{array}{llll}0.7498246803976775 & 0.2505137729889888 & 0.0651369668704198\end{array}$ $\begin{array}{llll}0.0001645500416413 & 0.5000292358778636 & 0.2094545286181233\end{array}$ $\begin{array}{lllll}0.5001645500416413 & 0.0000292358778634 & 0.2094545286181233\end{array}$ $\begin{array}{llll}0.2500959852712041 & 0.7498783506309599 & 0.3536229838618800\end{array}$ $\begin{array}{llll}0.7500959852712038 & 0.2498783506309595 & 0.3536229838618800\end{array}$ $\begin{array}{llll}-0.0001371949892787 & 0.4998394183615758 & 0.4980574774096541\end{array}$ $\begin{array}{llll}0.4998628050107213 & -0.0001605816384242 & 0.4980574774096541\end{array}$ $\begin{array}{llll}0.5171276671120684 & 0.1370730409929963 & 0.1059598948692713\end{array}$ $\begin{array}{llll}0.0171277261120692 & 0.6370730549929902 & 0.1059598948692713\end{array}$ $\begin{array}{llll}0.2673993414922523 & 0.8869095758638599 & 0.2501368687016219\end{array}$ $\begin{array}{llll}0.7673993414922520 & 0.3869095758638593 & 0.2501368687016219\end{array}$ $\begin{array}{llll}0.5177914714400556 & 0.1369941107395651 & 0.3941160069126952\end{array}$ $\begin{array}{llll}0.0177915304400569 & 0.6369941247395590 & 0.3941160069126952\end{array}$ $\begin{array}{llll}0.2683347141720304 & 0.8861730715369313 & 0.5389389927557419\end{array}$ $\begin{array}{llll}0.7683347141720306 & 0.3861730715369318 & 0.5389389927557419\end{array}$ $\begin{array}{llll}0.3636991994554167 & 0.9817975077614834 & 0.0243290393135025\end{array}$ $\begin{array}{lllll}0.8636991994554171 & 0.4817975077614835 & 0.0243290393135025\end{array}$ $\begin{array}{llll}0.1130486350334040 & 0.7319850135383910 & 0.1690187776366811\end{array}$ $\begin{array}{lllll}0.6130486280334071 & 0.2319849985383857 & 0.1690187776366811\end{array}$ $\begin{array}{llll}0.3629876027873057 & 0.9822440846749585 & 0.3131345610394079\end{array}$ $\begin{array}{llll}0.8629876027873058 & 0.4822440846749581 & 0.3131345610394079\end{array}$ $\begin{array}{llll}0.1129725738430085 & 0.7326142686344501 & 0.4572565602024776\end{array}$ $\begin{array}{llll}0.6129725668430112 & 0.2326142536344451 & 0.4572565602024776\end{array}$ $\begin{array}{lllll}0.1308369506660995 & 0.5109644566776085 & 0.0201637833531781\end{array}$ $\begin{array}{llll}0.6308369506660997 & 0.0109645166776060 & 0.0201637833531781\end{array}$ $\begin{array}{llll}0.8798397837291408 & 0.2607608345189835 & 0.1650033101809674\end{array}$ $\begin{array}{llll}0.3798397837291406 & 0.7607608045189882 & 0.1650033101809674\end{array}$ $\begin{array}{llll}0.1298542673282382 & 0.5105094295599865 & 0.3090260023633002\end{array}$ $\begin{array}{llll}0.6298542673282380 & 0.0105094895599844 & 0.3090260023633002\end{array}$ $\begin{array}{llll}0.8799216667893147 & 0.2602949092333234 & 0.4531634417335408\end{array}$ $\begin{array}{llll}0.3799216667893150 & 0.7602948792333278 & 0.4531634417335408\end{array}$ $\begin{array}{llll}0.9893627425461327 & 0.3703200987543939 & 0.1101087995792872\end{array}$ $\begin{array}{lllll}0.4893627125461371 & 0.8703200987543939 & 0.1101087995792872\end{array}$ $\begin{array}{llll}0.7395367655683565 & 0.1199533921488340 & 0.2540793314763372\end{array}$ $\begin{array}{lllll}0.2395367955683587 & 0.6199533991488311 & 0.2540793314763372\end{array}$ $\begin{array}{llll}0.9895556977418161 & 0.3701311333267873 & 0.3982224352521325\end{array}$ $\begin{array}{lllll}0.4895556677418204 & 0.8701311333267877 & 0.3982224352521325\end{array}$ $\begin{array}{llll}0.7395874122232149 & 0.1190688439981528 & 0.5431232341153851\end{array}$ $\begin{array}{llll}0.2395874422232175 & 0.6190688509981500 & 0.5431232341153851\end{array}$ $\begin{array}{llll}0.9890355433223915 & 0.8691630493339003 & 0.0201637833531781\end{array}$ $\begin{array}{lllll}0.4890355133223965 & 0.3691630493339007 & 0.0201637833531781\end{array}$ $\begin{array}{llll}0.7392391354810139 & 0.6201602162708592 & 0.1650033101809674\end{array}$ $\begin{array}{llll}0.2392391654810165 & 0.1201602162708593 & 0.1650033101809674\end{array}$ 
$\begin{array}{llll}0.9894905704400135 & 0.8701457326717620 & 0.3090260023633002\end{array}$ $\begin{array}{llll}0.4894905404400179 & 0.3701457326717619 & 0.3090260023633002\end{array}$ $\begin{array}{llll}0.7397050607666743 & 0.6200783332106853 & 0.4531634417335408\end{array}$ $\begin{array}{llll}0.2397050907666766 & 0.1200783332106850 & 0.4531634417335408\end{array}$ $\begin{array}{lllll}0.1296799012456064 & 0.0106373174538654 & 0.1101087995792872\end{array}$ $\begin{array}{llll}0.6296799012456061 & 0.5106372574538673 & 0.1101087995792872\end{array}$ $\begin{array}{llll}0.8800466008511689 & 0.7604631744316457 & 0.2540793314763372\end{array}$ $\begin{array}{llll}0.3800466008511689 & 0.2604632344316440 & 0.2540793314763372\end{array}$ $\begin{array}{llll}0.1298688666732127 & 0.0104443622581819 & 0.3982224352521325\end{array}$ $\begin{array}{llll}0.6298688666732123 & 0.5104443022581839 & 0.3982224352521325\end{array}$ $\begin{array}{llll}0.8809311490018500 & 0.7604125277767873 & 0.5431232341153851\end{array}$ $\begin{array}{llll}0.3809311490018502 & 0.2604125877767849 & 0.5431232341153851\end{array}$ $\begin{array}{llll}0.5182024922385166 & 0.6363008005445829 & 0.0243290393135025\end{array}$ $\begin{array}{llll}0.0182025512385178 & 0.1363007865445892 & 0.0243290393135025\end{array}$ $\begin{array}{llll}0.2680149864616093 & 0.3869513719665930 & 0.1690187776366811\end{array}$ $\begin{array}{llll}0.7680149864616090 & 0.8869513719665929 & 0.1690187776366811\end{array}$ $\begin{array}{llll}0.5177559153250415 & 0.6370123972126942 & 0.3131345610394079\end{array}$ $\begin{array}{llll}0.0177559743250433 & 0.1370123832127003 & 0.3131345610394079\end{array}$ $\begin{array}{llll}0.2673857313655499 & 0.3870274331569886 & 0.4572565602024776\end{array}$ $\begin{array}{llll}0.7673857313655499 & 0.8870274331569888 & 0.4572565602024776\end{array}$ $\begin{array}{lllll}0.3629269450070096 & 0.4828723328879321 & 0.1059598948692713\end{array}$ $\begin{array}{llll}0.8629269450070098 & 0.9828723328879316 & 0.1059598948692713\end{array}$ $\begin{array}{llll}0.1130904311361377 & 0.2326006435077429 & 0.2501368687016219\end{array}$ $\begin{array}{llll}0.6130904241361401 & 0.7326006585077480 & 0.2501368687016219\end{array}$ $\begin{array}{llll}0.3630058752604408 & 0.4822085285599444 & 0.3941160069126952\end{array}$ $\begin{array}{llll}0.8630058752604410 & 0.9822085285599444 & 0.3941160069126952\end{array}$ $\begin{array}{llll}0.1138269354630654 & 0.2316652708279646 & 0.5389389927557419\end{array}$ $\begin{array}{llll}0.6138269284630687 & 0.7316652858279694 & 0.5389389927557419\end{array}$ $\begin{array}{llll}0.4404525625552871 & 0.1632904178492680 & 0.0817870587461595\end{array}$ $\begin{array}{llll}0.9404525925552899 & 0.6632904478492704 & 0.0817870587461595\end{array}$ $\begin{array}{llll}0.1903480397599214 & 0.9134467516173652 & 0.2260966514862646\end{array}$ $\begin{array}{llll}0.6903480547599259 & 0.4134467216173624 & 0.2260966514862646\end{array}$ $\begin{array}{llll}0.4406152671297024 & 0.1631064470109028 & 0.3701615879271388\end{array}$ $\begin{array}{llll}0.9406152971297046 & 0.6631064770109053 & 0.3701615879271388\end{array}$ $\begin{array}{llll}0.1902933323794916 & 0.9132278875856999 & 0.5148843392399952\end{array}$ $\begin{array}{llll}0.6902933473794962 & 0.4132278575856971 & 0.5148843392399952\end{array}$ $\begin{array}{lllll}0.3363697440654653 & 0.0599986800658574 & 0.0483487880671202\end{array}$ $\begin{array}{llll}0.8363697140654632 & 0.5599986610658595 & 0.0483487880671202\end{array}$ $\begin{array}{llll}0.0870501576423468 & 0.8090951462753960 & 0.1929804285825509\end{array}$ $\begin{array}{llll}0.5870501356423454 & 0.3090951762753990 & 0.1929804285825509\end{array}$ $\begin{array}{llll}0.3369717574204186 & 0.0593588488735728 & 0.3370904744990318\end{array}$ $\begin{array}{llll}0.8369717274204163 & 0.5593588298735753 & 0.3370904744990318\end{array}$ $\begin{array}{llll}0.0874551535575498 & 0.8091611470482086 & 0.4813963196014951\end{array}$ $\begin{array}{llll}0.5874551315575478 & 0.3091611770482108 & 0.4813963196014951\end{array}$ $\begin{array}{lllll}0.1420124408937998 & 0.4156573774568724 & 0.0346684313487571\end{array}$ $\begin{array}{llll}0.6420124708937949 & 0.9156574074568676 & 0.0346684313487571\end{array}$ $\begin{array}{llll}0.8899864965940626 & 0.1672823938007742 & 0.1795623059056209\end{array}$ $\begin{array}{llll}0.3899864665940670 & 0.6672824088007719 & 0.1795623059056209\end{array}$ $\begin{array}{llll}0.1400134156130325 & 0.4172793067799465 & 0.3237483320206562\end{array}$ $\begin{array}{llll}0.6400134456130280 & 0.9172793367799420 & 0.3237483320206562\end{array}$ $\begin{array}{llll}0.8905156822036960 & 0.1665703258399294 & 0.4677614366214212\end{array}$ $\begin{array}{lllll}0.3905156522037005 & 0.6665703408399277 & 0.4677614366214212\end{array}$ $\begin{array}{llll}0.0830323582594932 & 0.3598775798405254 & 0.0954953211384069\end{array}$ $\begin{array}{llll}0.5830323432594955 & 0.8598775498405294 & 0.0954953211384069\end{array}$ $\begin{array}{llll}0.8328380095560162 & 0.1097259438623482 & 0.2393768970796363\end{array}$ $\begin{array}{llll}0.3328380395560114 & 0.6097259218623533 & 0.2393768970796363\end{array}$ 
$\begin{array}{llll}0.0829362754871290 & 0.3598101711144625 & 0.3835583948554613\end{array}$ $\begin{array}{llll}0.5829362604871311 & 0.8598101411144669 & 0.3835583948554613\end{array}$ $\begin{array}{llll}0.8347623713591198 & 0.1077820854123673 & 0.5284981022120414\end{array}$ $\begin{array}{llll}0.3347624013591156 & 0.6077820634123723 & 0.5284981022120414\end{array}$ $\begin{array}{llll}0.0843426075431300 & 0.8579875291062051 & 0.0346684313487571\end{array}$ $\begin{array}{llll}0.5843425925431324 & 0.3579875591062003 & 0.0346684313487571\end{array}$ $\begin{array}{llll}0.8327175911992281 & 0.6100135034059374 & 0.1795623059056209\end{array}$ $\begin{array}{lllll}0.3327176211992235 & 0.1100135624059317 & 0.1795623059056209\end{array}$ $\begin{array}{llll}0.0827206782200557 & 0.8599865543869720 & 0.3237483320206562\end{array}$ $\begin{array}{llll}0.5827206632200580 & 0.3599865843869674 & 0.3237483320206562\end{array}$ $\begin{array}{llll}0.8334296591600723 & 0.6094843177963040 & 0.4677614366214212\end{array}$ $\begin{array}{llll}0.3334296891600683 & 0.1094843767962984 & 0.4677614366214212\end{array}$ $\begin{array}{llll}0.1401224201594746 & 0.9169676567405045 & 0.0954953211384069\end{array}$ $\begin{array}{llll}0.6401224501594706 & 0.4169676267405091 & 0.0954953211384069\end{array}$ $\begin{array}{llll}0.8902740781376467 & 0.6671619904439838 & 0.2393768970796363\end{array}$ $\begin{array}{llll}0.3902740481376510 & 0.1671619904439838 & 0.2393768970796363\end{array}$ $\begin{array}{llll}0.1401898288855375 & 0.9170637395128689 & 0.3835583948554613\end{array}$ $\begin{array}{lllll}0.6401898588855331 & 0.4170637095128732 & 0.3835583948554613\end{array}$ $\begin{array}{llll}0.8922179365876277 & 0.6652376286408802 & 0.5284981022120414\end{array}$ $\begin{array}{llll}0.3922179065876320 & 0.1652376286408799 & 0.5284981022120414\end{array}$ $\begin{array}{llll}0.4400013089341382 & 0.6636302859345368 & 0.0483487880671202\end{array}$ $\begin{array}{llll}0.9400013389341405 & 0.1636302559345347 & 0.0483487880671202\end{array}$ $\begin{array}{llll}0.1909048387245988 & 0.4129498343576526 & 0.1929804285825509\end{array}$ $\begin{array}{lllll}0.6909048537246040 & 0.9129498643576546 & 0.1929804285825509\end{array}$ $\begin{array}{llll}0.4406411401264229 & 0.6630282725795837 & 0.3370904744990318\end{array}$ $\begin{array}{llll}0.9406411701264247 & 0.1630282425795814 & 0.3370904744990318\end{array}$ $\begin{array}{lllll}0.1908388379517867 & 0.4125448384424495 & 0.4813963196014951\end{array}$ $\begin{array}{llll}0.6908388529517914 & 0.9125448684424522 & 0.4813963196014951\end{array}$ $\begin{array}{llll}0.3367095821507318 & 0.5595474074447101 & 0.0817870587461595\end{array}$ $\begin{array}{llll}0.8367095521507296 & 0.0595474264447082 & 0.0817870587461595\end{array}$ $\begin{array}{llll}0.0865532703826369 & 0.3096519752400764 & 0.2260966514862646\end{array}$ $\begin{array}{llll}0.5865532483826348 & 0.8096519452400741 & 0.2260966514862646\end{array}$ $\begin{array}{lllll}0.3368935529890970 & 0.5593847028702954 & 0.3701615879271388\end{array}$ $\begin{array}{llll}0.8368935229890947 & 0.0593847218702932 & 0.3701615879271388\end{array}$ $\begin{array}{llll}0.0867721344143023 & 0.3097066826205064 & 0.5148843392399952\end{array}$ $\begin{array}{lllll}0.5867721124143001 & 0.8097066526205038 & 0.5148843392399952\end{array}$ $\begin{array}{llll}0.6107841554592538 & 0.1032802698994243 & 0.0973477224309301\end{array}$ $\begin{array}{llll}0.1107842144592548 & 0.6032802698994241 & 0.0973477224309301\end{array}$ $\begin{array}{llll}0.3610772859674266 & 0.8526706361778568 & 0.2414207352453246\end{array}$ $\begin{array}{lllll}0.8610772859674263 & 0.3526706361778571 & 0.2414207352453246\end{array}$ $\begin{array}{lllll}0.6113805827711161 & 0.1027192024083752 & 0.3852869267078089\end{array}$ $\begin{array}{llll}0.1113806417711176 & 0.6027192024083750 & 0.3852869267078089\end{array}$ $\begin{array}{llll}0.3613165432880395 & 0.8532663943792647 & 0.5297824960389613\end{array}$ $\begin{array}{lllll}0.8613165432880391 & 0.3532663943792649 & 0.5297824960389613\end{array}$ $\begin{array}{llll}0.3965913175851172 & 0.8888219934543712 & 0.0334873792807646\end{array}$ $\begin{array}{lllll}0.8965913175851171 & 0.3888219934543714 & 0.0334873792807646\end{array}$ $\begin{array}{llll}0.1474780768988213 & 0.6383160191678656 & 0.1778164964839039\end{array}$ $\begin{array}{llll}0.6474780768988214 & 0.1383160191678655 & 0.1778164964839039\end{array}$ $\begin{array}{llll}0.3974071643345478 & 0.8885941431916489 & 0.3219326385862123\end{array}$ $\begin{array}{llll}0.8974071643345478 & 0.3885941431916486 & 0.3219326385862123\end{array}$ $\begin{array}{llll}0.1472940397508478 & 0.6388445372143424 & 0.4659132278014332\end{array}$ $\begin{array}{lllll}0.6472940397508476 & 0.1388445372143421 & 0.4659132278014332\end{array}$ $\begin{array}{llll}0.0514728256465406 & 0.5601359191357798 & 0.0032963834286809\end{array}$ $\begin{array}{llll}0.5514728546465393 & 0.0601358591357816 & 0.0032963834286809\end{array}$ $\begin{array}{llll}0.8006295693149360 & 0.3097255143417661 & 0.1479060804140549\end{array}$ $\begin{array}{llll}0.3006295993149384 & 0.8097255443417684 & 0.1479060804140549\end{array}$ 
$\begin{array}{llll}0.0504392980397484 & 0.5593032313379885 & 0.2919866737430892\end{array}$ $\begin{array}{lllll}0.5504393270397474 & 0.0593031713379911 & 0.2919866737430892\end{array}$ $\begin{array}{llll}0.8004603086012527 & 0.3091670727176428 & 0.4361489673660537\end{array}$ $\begin{array}{llll}0.3004603386012553 & 0.8091671027176451 & 0.4361489673660537\end{array}$ $\begin{array}{llll}0.9404400509170869 & 0.4497327428065089 & 0.1271376318370049\end{array}$ $\begin{array}{llll}0.4404400809170891 & 0.9497327138065100 & 0.1271376318370049\end{array}$ $\begin{array}{llll}0.6908809247580711 & 0.1992321680588138 & 0.2711576020090113\end{array}$ $\begin{array}{llll}0.1908808797580709 & 0.6992321390588150 & 0.2711576020090113\end{array}$ $\begin{array}{llll}0.9407595806287820 & 0.4495128422022063 & 0.4152626696748937\end{array}$ $\begin{array}{llll}0.4407596106287847 & 0.9495128132022073 & 0.4152626696748937\end{array}$ $\begin{array}{llll}0.6908615244391769 & 0.1983319130089966 & 0.5600276912244579\end{array}$ $\begin{array}{llll}0.1908614794391766 & 0.6983318840089978 & 0.5600276912244579\end{array}$ $\begin{array}{llll}0.9398640808642202 & 0.9485271453534607 & 0.0032963834286809\end{array}$ $\begin{array}{llll}0.4398641108642232 & 0.4485271743534595 & 0.0032963834286809\end{array}$ $\begin{array}{lllll}0.6902745156582295 & 0.6993703716850627 & 0.1479060804140549\end{array}$ $\begin{array}{lllll}0.1902744706582291 & 0.1993704306850567 & 0.1479060804140549\end{array}$ $\begin{array}{llll}0.9406967686620115 & 0.9495606729602526 & 0.2919866737430892\end{array}$ $\begin{array}{lllll}0.4406967986620136 & 0.4495607019602515 & 0.2919866737430892\end{array}$ $\begin{array}{lllll}0.6908329572823527 & 0.6995396323987459 & 0.4361489673660537\end{array}$ $\begin{array}{llll}0.1908329122823525 & 0.1995396913987401 & 0.4361489673660537\end{array}$ $\begin{array}{llll}0.0502672571934913 & 0.0595598890829155 & 0.1271376318370049\end{array}$ $\begin{array}{llll}0.5502672861934900 & 0.5595599490829131 & 0.1271376318370049\end{array}$ $\begin{array}{llll}0.8007678019411837 & 0.8091191352419268 & 0.2711576020090113\end{array}$ $\begin{array}{llll}0.3007678319411862 & 0.3091190752419290 & 0.2711576020090113\end{array}$ $\begin{array}{llll}0.0504871577977936 & 0.0592403593712202 & 0.4152626696748937\end{array}$ $\begin{array}{llll}0.5504871867977927 & 0.5592404193712180 & 0.4152626696748937\end{array}$ $\begin{array}{llll}0.8016680569910009 & 0.8091385355608209 & 0.5600276912244579\end{array}$ $\begin{array}{llll}0.3016680869910035 & 0.3091384755608231 & 0.5600276912244579\end{array}$ $\begin{array}{llll}0.6111780065456288 & 0.6034086824148829 & 0.0334873792807646\end{array}$ $\begin{array}{llll}0.1111780655456301 & 0.1034086824148829 & 0.0334873792807646\end{array}$ $\begin{array}{llll}0.3616839808321347 & 0.3525219231011785 & 0.1778164964839039\end{array}$ $\begin{array}{lllll}0.8616839808321344 & 0.8525219231011786 & 0.1778164964839039\end{array}$ $\begin{array}{llll}0.6114058568083511 & 0.6025928356654522 & 0.3219326385862123\end{array}$ $\begin{array}{llll}0.1114059158083527 & 0.1025928356654523 & 0.3219326385862123\end{array}$ $\begin{array}{llll}0.3611554627856577 & 0.3527059602491522 & 0.4659132278014332\end{array}$ $\begin{array}{llll}0.8611554627856576 & 0.8527059602491524 & 0.4659132278014332\end{array}$ $\begin{array}{lllll}0.3967197301005755 & 0.3892158445407465 & 0.0973477224309301\end{array}$ $\begin{array}{llll}0.8967197301005759 & 0.8892158445407462 & 0.0973477224309301\end{array}$ $\begin{array}{lllll}0.1473293638221428 & 0.1389227140325734 & 0.2414207352453246\end{array}$ $\begin{array}{lllll}0.6473293638221432 & 0.6389227140325737 & 0.2414207352453246\end{array}$ $\begin{array}{llll}0.3972807975916247 & 0.3886194172288840 & 0.3852869267078089\end{array}$ $\begin{array}{llll}0.8972807975916250 & 0.8886194172288839 & 0.3852869267078089\end{array}$ $\begin{array}{llll}0.1467336056207353 & 0.1386834567119606 & 0.5297824960389613\end{array}$ $\begin{array}{llll}0.6467336056207353 & 0.6386834567119609 & 0.5297824960389613\end{array}$ $\begin{array}{llll}0.4804276618878164 & 0.1513321141437730 & 0.1307368805453407\end{array}$ $\begin{array}{llll}0.9804276318878142 & 0.6513321291437707 & 0.1307368805453407\end{array}$ $\begin{array}{llll}0.2309727695784112 & 0.9014085906393290 & 0.2749602648037746\end{array}$ $\begin{array}{llll}0.7309727395784086 & 0.4014085906393291 & 0.2749602648037746\end{array}$ $\begin{array}{lllll}0.4816134282346740 & 0.1518221510416267 & 0.4189708354962670\end{array}$ $\begin{array}{llll}0.9816133982346716 & 0.6518221660416244 & 0.4189708354962670\end{array}$ $\begin{array}{llll}0.2335734101227912 & 0.8988675716261828 & 0.5637815812449696\end{array}$ $\begin{array}{lllll}0.7335733801227887 & 0.3988675716261831 & 0.5637815812449696\end{array}$ $0.35118700368763360 .0164646573965676-0.0005152984081657$ $0.85118700368763340 .5164646813965695-0.0005152984081657$ $\begin{array}{llll}0.0980060900816980 & 0.7682391420505346 & 0.1441710956348724\end{array}$ $\begin{array}{llll}0.5980060750817002 & 0.2682391120505321 & 0.1441710956348724\end{array}$ 
$\begin{array}{llll}0.3479256126468875 & 0.0185367422168792 & 0.2882800101401782\end{array}$ $\begin{array}{lllll}0.8479256126468874 & 0.5185367662168809 & 0.2882800101401782\end{array}$ $\begin{array}{lllll}0.0977050759655571 & 0.7694935171951628 & 0.4324692518011309\end{array}$ $\begin{array}{lllll}0.5977050609655593 & 0.2694934871951605 & 0.4324692518011309\end{array}$ $\begin{array}{lllll}0.1999881604813103 & 0.5277689562927420 & 0.0265516226776009\end{array}$ $\begin{array}{lllll}0.6999881454813124 & 0.0277688962927442 & 0.0265516226776009\end{array}$ $\begin{array}{lllll}0.9484621565296278 & 0.2782714737359225 & 0.1712734246027882\end{array}$ $\begin{array}{lllll}0.4484621865296231 & 0.7782714737359221 & 0.1712734246027882\end{array}$ $\begin{array}{lllll}0.1986130305569350 & 0.5280032970090114 & 0.3151092263516259\end{array}$ $\begin{array}{lllll}0.6986130155569372 & 0.0280032370090138 & 0.3151092263516259\end{array}$ $\begin{array}{lllll}0.94843248884744154 & 0.2780220631738695 & 0.4593473390547743\end{array}$ $\begin{array}{lllll}0.4484325188474105 & 0.7780220631738698 & 0.4593473390547743\end{array}$ $\begin{array}{lllll}0.9717467638839135 & 0.3017388888923729 & 0.1039156021629693\end{array}$ $\begin{array}{lllll}0.4717467638839136 & 0.8017389188923680 & 0.1039156021629693\end{array}$ $\begin{array}{lllll}0.7218537613115763 & 0.0513748376096527 & 0.2479307167729064\end{array}$ $\begin{array}{lllll}0.2218537613115761 & 0.5513748606096512 & 0.2479307167729064\end{array}$ $\begin{array}{lllll}0.9719704340753343 & 0.3014501445946597 & 0.3920934349632998\end{array}$ $\begin{array}{lllll}0.4719704340753340 & 0.8014501745946550 & 0.3920934349632998\end{array}$ $\begin{array}{lllll}0.7223848801655861 & 0.0501801884650397 & 0.5367743636101762\end{array}$ $\begin{array}{lllll}0.2223848801655860 & 0.5501802114650383 & 0.5367743636101762\end{array}$ $\begin{array}{lllll}0.9722310437072580 & 0.8000118545186876 & 0.0265516226776009\end{array}$ $\begin{array}{lllll}0.4722310437072579 & 0.3000118245186922 & 0.0265516226776009\end{array}$ $\begin{array}{lllll}0.7217285262640779 & 0.5515378434703722 & 0.1712734246027882\end{array}$ $\begin{array}{lllll}0.2217285262640774 & 0.0515378434703723 & 0.1712734246027882\end{array}$ $\begin{array}{lllll}0.9719967029909886 & 0.8013869844430628 & 0.3151092263516259\end{array}$ $\begin{array}{lllll}0.4719967029909884 & 0.3013869544430672 & 0.3151092263516259\end{array}$ $\begin{array}{lllll}0.7219779368261302 & 0.5515675111525846 & 0.4593473390547743\end{array}$ $\begin{array}{lllll}0.2219779368261306 & 0.0515675111525849 & 0.4593473390547743\end{array}$ $\begin{array}{lllll}0.1982610961076294 & 0.0282531761160887 & 0.1039156021629693\end{array}$ $\begin{array}{lllll}0.6982610811076320 & 0.5282532361160865 & 0.1039156021629693\end{array}$ $\begin{array}{lllll}0.9486251393903488 & 0.7781462386884237 & 0.2479307167729064\end{array}$ $\begin{array}{lllll}0.4486251693903445 & 0.2781461786884261 & 0.2479307167729064\end{array}$ $\begin{array}{lllll}0.1985498404053424 & 0.0280295059246681 & 0.3920934349632998\end{array}$ $\begin{array}{lllll}0.6985498254053450 & 0.5280295659246657 & 0.3920934349632998\end{array}$ $\begin{array}{lllll}0.9498197885349617 & 0.7776151198344139 & 0.5367743636101762\end{array}$ $\begin{array}{lllll}0.4498198185349573 & 0.2776150598344158 & 0.5367743636101762\end{array}$ $\begin{array}{llll}0.4835353486034327 & 0.6488129963123666 & -0.0005152984081657\end{array}$ $\begin{array}{llll}0.9835353186034305 & 0.1488129813123688 & -0.0005152984081657\end{array}$ $\begin{array}{llllll}0.2317608879494677 & 0.4019939249182996 & 0.1441710956348724\end{array}$ $\begin{array}{lllll}0.7317608579494654 & 0.9019939249182998 & 0.1441710956348724\end{array}$ $\begin{array}{lllll}0.4814632637831212 & 0.6520743873531126 & 0.2882800101401782\end{array}$ $\begin{array}{lllll}0.9814632337831191 & 0.1520743723531149 & 0.2882800101401782\end{array}$ $\begin{array}{lllll}0.2305065128048397 & 0.4022949390344406 & 0.4324692518011309\end{array}$ $\begin{array}{lllll}0.7305064828048372 & 0.9022949390344407 & 0.4324692518011309\end{array}$ $\begin{array}{lllll}0.3486678708562292 & 0.5195723681121858 & 0.1307368805453407\end{array}$ $\begin{array}{lllll}0.8486678708562293 & 0.0195723441121840 & 0.1307368805453407\end{array}$ $\begin{array}{lllll}0.0985914243606686 & 0.2690272304215887 & 0.2749602648037746\end{array}$ $\begin{array}{lllll}0.5985914093606710 & 0.7690272604215914 & 0.2749602648037746\end{array}$ $\begin{array}{lllll}0.3481778339583755 & 0.5183866017653284 & 0.4189708354962670\end{array}$ $\begin{array}{lllll}0.8481778339583756 & 0.0183865777653265 & 0.4189708354962670\end{array}$ $\begin{array}{lllll}0.1011324433738147 & 0.2664265898772089 & 0.5637815812449696\end{array}$ $\begin{array}{lllll}0.6011324283738172 & 0.7664266198772113 & 0.5637815812449696\end{array}$ 


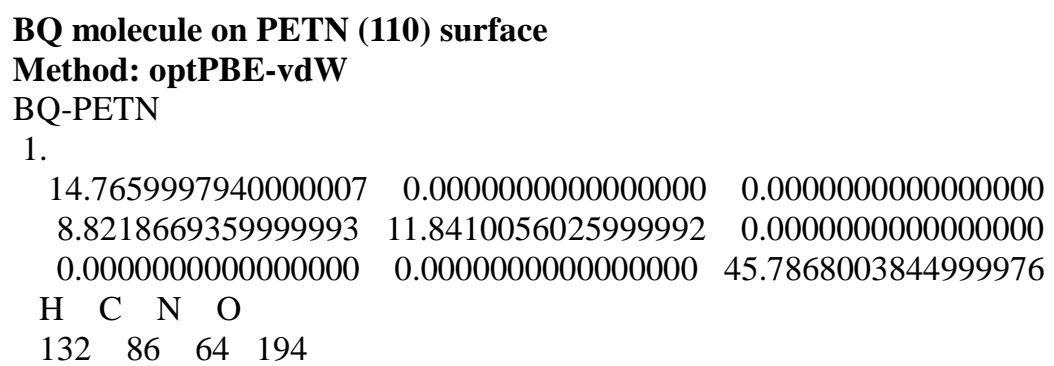

Direct

$\begin{array}{llll}0.3363315380178927 & 0.1259314724991004 & 0.0992562102406529\end{array}$

$\begin{array}{llll}0.8363566972487417 & 0.6258886985374553 & 0.0992554596762460\end{array}$ $\begin{array}{llll}0.0876726340884960 & 0.8756022915096282 & 0.2444052799265437\end{array}$ $\begin{array}{llll}0.5875232135822783 & 0.3757715739580320 & 0.2444514790820014\end{array}$ $\begin{array}{llll}0.3381184816915275 & 0.1257702544757032 & 0.3889013650899615\end{array}$ $\begin{array}{lllll}0.8382204183224791 & 0.6260632739431141 & 0.3889417709341326\end{array}$ $\begin{array}{llll}0.0850396869006297 & 0.8770000993201500 & 0.5354329708691411\end{array}$ $\begin{array}{llll}0.5904164362114410 & 0.3721840759437229 & 0.5328703562115199\end{array}$ $\begin{array}{lllll}0.3694060879657521 & 0.1653807200690654 & 0.0279511130328382\end{array}$ $\begin{array}{lllll}0.8695292899775994 & 0.6652930232267006 & 0.0279561626179444\end{array}$ $\begin{array}{llll}0.1233017713817356 & 0.9125003234581092 & 0.1736764785946975\end{array}$ $\begin{array}{llll}0.6232267726116754 & 0.4125560803092574 & 0.1737032023337800\end{array}$ $\begin{array}{lllll}0.3736374482271884 & 0.1623290969686925 & 0.3181673062912276\end{array}$ $\begin{array}{llll}0.8739977589230583 & 0.6621513432066193 & 0.3181228582218198\end{array}$ $\begin{array}{llll}0.1211083556360731 & 0.9135794993864482 & 0.4638861345138946\end{array}$ $\begin{array}{lllll}0.6260513361310984 & 0.4097361742870451 & 0.4628032987706681\end{array}$ $\begin{array}{llll}0.2012836786818484 & 0.4186051323196328 & 0.0744859406123508\end{array}$ $\begin{array}{lllll}0.7012513501576251 & 0.9185763925327554 & 0.0744934432722904\end{array}$ $\begin{array}{lllll}0.9556446448961355 & 0.1663985052998827 & 0.2196456975041543\end{array}$ $\begin{array}{lllll}0.4556086253147720 & 0.6664712471018397 & 0.2196488695157001\end{array}$ $\begin{array}{llll}0.2057015198850574 & 0.4165491932657674 & 0.3639939503525109\end{array}$ $\begin{array}{llll}0.7062575448554523 & 0.9164819691139321 & 0.3639136990120341\end{array}$ $\begin{array}{llll}0.9534223221225776 & 0.1672997955297987 & 0.5098061544535010\end{array}$ $\begin{array}{llll}0.4596604887231015 & 0.6642044505254218 & 0.5084305283779612\end{array}$ $\begin{array}{llll}0.0808176520751422 & 0.2962390092488278 & 0.0541829434693247\end{array}$ $\begin{array}{llll}0.5809573514641735 & 0.7960578797036288 & 0.0541689528654185\end{array}$ $\begin{array}{llllll}0.8332084933028095 & 0.0437421038610345 & 0.1987863812543436\end{array}$ $\begin{array}{lllll}0.3330648782476013 & 0.5439560041738937 & 0.1988022380994901\end{array}$ $\begin{array}{lllll}0.0833467470822119 & 0.2938536847289315 & 0.3433595677278139\end{array}$ $\begin{array}{llll}0.5839112137597469 & 0.7936145419274989 & 0.3432509131430684\end{array}$ $\begin{array}{lllll}0.8287415039317144 & 0.0518253850857357 & 0.4899593293246972\end{array}$ $\begin{array}{llll}0.3360898962224735 & 0.5409067536991948 & 0.4871412905691744\end{array}$ $\begin{array}{llll}0.0814663888379555 & 0.7987532726257058 & 0.0744999916031268\end{array}$ $\begin{array}{lllll}0.5813900244219695 & 0.2987717115637785 & 0.0744589593893332\end{array}$ $\begin{array}{llll}0.8336792224598950 & 0.5443110341724333 & 0.2196865455397926\end{array}$ $\begin{array}{llll}0.3337286426149694 & 0.0443100462469583 & 0.2196783512728610\end{array}$ $\begin{array}{llll}0.0832345852386399 & 0.7947635269444081 & 0.3640505307403512\end{array}$ $\begin{array}{llll}0.5837280279722394 & 0.2939744764631293 & 0.3641579843010121\end{array}$ $\begin{array}{llllll}0.8335193675979958 & 0.5464824255983561 & 0.5094032389252748\end{array}$ $\begin{array}{lllll}0.3325202389632744 & 0.0403813264221617 & 0.5086340959066752\end{array}$ $\begin{array}{llll}0.2039834873208168 & 0.9189525732913962 & 0.0541668514359325\end{array}$ $\begin{array}{llll}0.7038989994584853 & 0.4190256739378458 & 0.0541860832402407\end{array}$ $\begin{array}{llll}0.9563799846399206 & 0.6667918127788180 & 0.1988302444702540\end{array}$ $\begin{array}{lllll}0.4564648293556585 & 0.1667631264892891 & 0.1988186123669175\end{array}$ $\begin{array}{llll}0.2067552145794891 & 0.9163388427619156 & 0.3433775962617166\end{array}$ $\begin{array}{llll}0.7062923628468076 & 0.4162455918832043 & 0.3433556380844754\end{array}$ 
$\begin{array}{llll}0.9543197735220117 & 0.6681055958805232 & 0.4891772523116266\end{array}$ $\begin{array}{llll}0.4531517643942376 & 0.1606090830706770 & 0.4880501132692809\end{array}$ $\begin{array}{lllll}0.3348201689467564 & 0.6303783292306656 & 0.0279528751607562\end{array}$ $\begin{array}{lllll}0.8348766386447797 & 0.1303732103324244 & 0.0279803778923217\end{array}$ $\begin{array}{lllll}0.0874677885597768 & 0.3767972458501507 & 0.1736935037989569\end{array}$ $\begin{array}{llll}0.5875075808577103 & 0.8767326935195037 & 0.1736988208604493\end{array}$ $\begin{array}{lllll}0.3376109118686019 & 0.6261365626178715 & 0.3182473486931988\end{array}$ $\begin{array}{llll}0.8376299529857330 & 0.1258687811710547 & 0.3182227493718315\end{array}$ $\begin{array}{llll}0.0866183668183805 & 0.3772532765095233 & 0.4634702425960329\end{array}$ $\begin{array}{llll}0.5903583232125874 & 0.8662343197997914 & 0.4641046089562729\end{array}$ $\begin{array}{llll}0.3740965114562951 & 0.6634277463175425 & 0.0992669958447467\end{array}$ $\begin{array}{llll}0.8740295463670591 & 0.1634744897648986 & 0.0992762544980256\end{array}$ $\begin{array}{llll}0.1243930238635105 & 0.4122398205186020 & 0.2444469047685177\end{array}$ $\begin{array}{llll}0.6244919854284604 & 0.9122585878212940 & 0.2444324091564984\end{array}$ $\begin{array}{llll}0.3737905875070601 & 0.6619953296686384 & 0.3890548054519252\end{array}$ $\begin{array}{llll}0.8742774266807061 & 0.1620128059745810 & 0.3890363900950660\end{array}$ $\begin{array}{llll}0.1245131476752784 & 0.4130110280461221 & 0.5347938221539024\end{array}$ $\begin{array}{llll}0.6205325794120850 & 0.9093008135894346 & 0.5351607937801399\end{array}$ $\begin{array}{llll}0.2991690385583152 & 0.2625128801477137 & 0.1079614472821099\end{array}$ $\begin{array}{llll}0.7992591608648939 & 0.7624504159102339 & 0.1079567400160035\end{array}$ $\begin{array}{lllll}0.0504582021995274 & 0.0123437040856849 & 0.2529809608330034\end{array}$ $\begin{array}{llll}0.5502554358526159 & 0.5125231945145627 & 0.2530083259931883\end{array}$ $\begin{array}{llll}0.3006913002380545 & 0.2626236495081624 & 0.3974317593932829\end{array}$ $\begin{array}{llll}0.8009314053203592 & 0.7629039311354981 & 0.3973637537748297\end{array}$ $\begin{array}{lllll}0.0508734036635413 & 0.0130936828577649 & 0.5432765200213896\end{array}$ $\begin{array}{lllll}0.5488450160655831 & 0.5098373286184102 & 0.5425277781595857\end{array}$ $\begin{array}{llll}0.2322865329817587 & 0.2016461852223201 & 0.0203677077988283\end{array}$ $\begin{array}{llll}0.7323910042505652 & 0.7016389924927404 & 0.0203549517967069\end{array}$ $\begin{array}{llll}0.9862956267592149 & 0.9500245099342216 & 0.1652530665601042\end{array}$ $\begin{array}{llll}0.4862333571714937 & 0.4500437963597745 & 0.1652845762642724\end{array}$ $\begin{array}{llll}0.2366828132140604 & 0.1997156260462114 & 0.3097566154129998\end{array}$ $\begin{array}{llll}0.7370422964390865 & 0.6994990009552629 & 0.3097640414367515\end{array}$ $\begin{array}{llll}0.9839145123989869 & 0.9511081211647187 & 0.4558092441015264\end{array}$ $\begin{array}{llll}0.4888520018319908 & 0.4483814759158269 & 0.4541867445656172\end{array}$ $\begin{array}{llll}0.3080402847329746 & 0.3448346130017251 & 0.0469980741335101\end{array}$ $\begin{array}{lllll}0.8080269810061579 & 0.8448750221276341 & 0.0470064669573422\end{array}$ $\begin{array}{llll}0.0578196741360595 & 0.0941675945297972 & 0.1910333600965061\end{array}$ $\begin{array}{llll}0.5577580825713248 & 0.5941762365575062 & 0.1910344494670123\end{array}$ $\begin{array}{llll}0.3078792517719972 & 0.3441138476362672 & 0.3353938893104599\end{array}$ $\begin{array}{llll}0.8083437535471245 & 0.8439938482538648 & 0.3352986404201540\end{array}$ $\begin{array}{lllll}0.0577831584365021 & 0.0935386311080096 & 0.4817636949865097\end{array}$ $\begin{array}{llll}0.5607503580898888 & 0.5914554371949047 & 0.4795565064579253\end{array}$ $\begin{array}{lllll}0.1542952444644904 & 0.1930120963022924 & 0.0824949926716955\end{array}$ $\begin{array}{llll}0.6544447208748612 & 0.6928780425936973 & 0.0824852521089215\end{array}$ $\begin{array}{llll}0.9057974645466491 & 0.9418905313935930 & 0.2274542916339135\end{array}$ $\begin{array}{llll}0.4056814261257637 & 0.4419266347424646 & 0.2274333858043573\end{array}$ $\begin{array}{llll}0.1561722076161207 & 0.1916379075428652 & 0.3719371713241255\end{array}$ $\begin{array}{llll}0.6567483956857039 & 0.6914543302846962 & 0.3718408781989119\end{array}$ $\begin{array}{lllll}0.9047473783708369 & 0.9392719259853164 & 0.5159148054669931\end{array}$ $\begin{array}{llll}0.4051499758102683 & 0.4439912747099499 & 0.5169342124765827\end{array}$ $\begin{array}{llll}0.1550967973614576 & 0.6919494491726316 & 0.0470121313151271\end{array}$ $\begin{array}{llll}0.6551167545584043 & 0.1919946451392935 & 0.0469714182073285\end{array}$ $\begin{array}{llll}0.9058847291865502 & 0.4422480774099951 & 0.1910490417190640\end{array}$ $\begin{array}{llll}0.4059531177045983 & 0.9422091802680075 & 0.1910486669166369\end{array}$ $\begin{array}{llll}0.1555848159721440 & 0.6922873306149174 & 0.3355065450376864\end{array}$ $\begin{array}{llll}0.6559291394463009 & 0.1917205439361053 & 0.3355674516707345\end{array}$ 
$\begin{array}{llll}0.9064729889089997 & 0.4423245167051463 & 0.4813112838726497\end{array}$ $\begin{array}{llll}0.4044929078378572 & 0.9379977247880277 & 0.4800644472022087\end{array}$ $\begin{array}{llll}0.3071791022054508 & 0.8454858670348793 & 0.0824773804731315\end{array}$ $\begin{array}{llll}0.8071244427297664 & 0.3455363947243260 & 0.0824929003412878\end{array}$ $\begin{array}{lllll}0.0582392018617632 & 0.5941338829515362 & 0.2274965475689477\end{array}$ $\begin{array}{llll}0.5582614476735738 & 0.0941452963935936 & 0.2274973016015985\end{array}$ $\begin{array}{llll}0.3084525900442835 & 0.8435313423390294 & 0.3720715441071362\end{array}$ $\begin{array}{llll}0.8089550729747893 & 0.3432363131419138 & 0.3718381232442640\end{array}$ $\begin{array}{llll}0.0620461456921914 & 0.5930706590898605 & 0.5164205818355792\end{array}$ $\begin{array}{llll}0.5628504961112791 & 0.0859618188355309 & 0.5147162233493698\end{array}$ $\begin{array}{lllll}0.2984693236547697 & 0.7675243912890087 & 0.0203584979345501\end{array}$ $\begin{array}{lllll}0.7984850611322769 & 0.2675054884908890 & 0.0203683328353620\end{array}$ $\begin{array}{llll}0.0499604412575426 & 0.5138096018880676 & 0.1652937909354954\end{array}$ $\begin{array}{llll}0.5500069861630648 & 0.0137235555870026 & 0.1652764440016375\end{array}$ $\begin{array}{llll}0.3003091608574150 & 0.7630327409655652 & 0.3097965983631431\end{array}$ $\begin{array}{llll}0.8003493032496301 & 0.2627513332790418 & 0.3097805432815540\end{array}$ $\begin{array}{llll}0.0503489024242253 & 0.5138005247052748 & 0.4552442817377444\end{array}$ $\begin{array}{llll}0.5518080691540497 & 0.0028732132191176 & 0.4547965525853833\end{array}$ $\begin{array}{llll}0.2374548641010034 & 0.7007271583109159 & 0.1079483858912696\end{array}$ $\begin{array}{llll}0.7373715624942921 & 0.2007912687257043 & 0.1079569017267355\end{array}$ $\begin{array}{llll}0.9875895320072364 & 0.4495831847431868 & 0.2530009994055188\end{array}$ $\begin{array}{llll}0.4877077952242989 & 0.9496157721405153 & 0.2530050107956140\end{array}$ $\begin{array}{llll}0.2369308230605819 & 0.6991827727635553 & 0.3974300741092757\end{array}$ $\begin{array}{llll}0.7375187491470498 & 0.1991079537321149 & 0.3974880805226396\end{array}$ $\begin{array}{lllll}0.9875987070183512 & 0.4494734543005071 & 0.5428822656683198\end{array}$ $\begin{array}{lllll}0.4832203252654433 & 0.9457657090871587 & 0.5425946975246870\end{array}$ $\begin{array}{lllll}0.4948172740789134 & 0.6336796690364406 & 0.6436888513364839\end{array}$ $\begin{array}{llll}0.5455536477130618 & 0.4866077532234434 & 0.6805989288928047\end{array}$ $\begin{array}{llll}0.5694790360832463 & 0.3111609318924750 & 0.6674092695442140\end{array}$ $\begin{array}{llll}0.5343165080648735 & 0.2763556956374707 & 0.6168725557469013\end{array}$ $\begin{array}{llll}0.3289885069881890 & 0.1994061072123895 & 0.0906765352485601\end{array}$ $\begin{array}{llll}0.8290665923530840 & 0.6993345715740932 & 0.0906741383658439\end{array}$ $\begin{array}{lllll}0.0800956853116553 & 0.9490961223613511 & 0.2357493777798171\end{array}$ $\begin{array}{llll}0.5799415892864045 & 0.4492451595248448 & 0.2357824913529314\end{array}$ $\begin{array}{llll}0.3304269201668174 & 0.1992862227272726 & 0.3802143362869860\end{array}$ $\begin{array}{llll}0.8307206622260808 & 0.6994039035242918 & 0.3801943591058481\end{array}$ $\begin{array}{lllll}0.0796796686941110 & 0.9488003043703481 & 0.5262974102552959\end{array}$ $\begin{array}{llll}0.5809852723253491 & 0.4474217274740705 & 0.5249243978610708\end{array}$ $\begin{array}{llll}0.2973311058406768 & 0.1715419670069885 & 0.0371963991905325\end{array}$ $\begin{array}{llll}0.7974154315822468 & 0.6715057530558960 & 0.0371905245278676\end{array}$ $\begin{array}{llll}0.0498942022304411 & 0.9200845966031745 & 0.1824005041859621\end{array}$ $\begin{array}{lllll}0.5498345882713168 & 0.4201240919170831 & 0.1824317123270231\end{array}$ $\begin{array}{llll}0.3002478496347578 & 0.1698952034343743 & 0.3269064016742497\end{array}$ $\begin{array}{llll}0.8006459881604300 & 0.6697539615244811 & 0.3268966819907890\end{array}$ $\begin{array}{llll}0.0482430211711840 & 0.9204734228896019 & 0.4728027508849609\end{array}$ $\begin{array}{llll}0.5520855134867473 & 0.4181434363350828 & 0.4714450595209336\end{array}$ $\begin{array}{llll}0.2302716267367467 & 0.3616359291745713 & 0.0556772211939386\end{array}$ $\begin{array}{llll}0.7302854868603211 & 0.8616131171906085 & 0.0556840844977738\end{array}$ $\begin{array}{lllll}0.9813910086665668 & 0.1107724410311629 & 0.2006593343963678\end{array}$ $\begin{array}{llll}0.4813131356429209 & 0.6108283569059215 & 0.2006666932987400\end{array}$ $\begin{array}{llll}0.2314641681838100 & 0.3607566816241983 & 0.3450433646155011\end{array}$ $\begin{array}{llll}0.7319507873390853 & 0.8606739154113292 & 0.3449715263247587\end{array}$ $\begin{array}{llll}0.9806154826913650 & 0.1105728669052356 & 0.4910008326278460\end{array}$ $\begin{array}{llll}0.4845202411780090 & 0.6086016142202068 & 0.4894798864029454\end{array}$ $\begin{array}{llll}0.1375593324690548 & 0.2696195149415871 & 0.0729547853804509\end{array}$ $\begin{array}{llll}0.6376551705709536 & 0.7695133099800979 & 0.0729461547778910\end{array}$ 
$\begin{array}{llll}0.8889800368000177 & 0.0182808796532194 & 0.2177350094028535\end{array}$ $\begin{array}{llll}0.3888731120427270 & 0.5183618396344331 & 0.2177478671435749\end{array}$ $\begin{array}{llll}0.1393322988278427 & 0.2681191693360321 & 0.3622703195506756\end{array}$ $\begin{array}{llll}0.6397423502354310 & 0.7680232283956074 & 0.3621918764716631\end{array}$ $\begin{array}{lllll}0.8879888175197105 & 0.0182827702307822 & 0.5083547370639199\end{array}$ $\begin{array}{llll}0.3895458822006942 & 0.5186414052564802 & 0.5064503952530550\end{array}$ $\begin{array}{lllll}0.1384050557916307 & 0.7696738386726014 & 0.0556818683911017\end{array}$ $\begin{array}{llll}0.6383968995250430 & 0.2697100708180672 & 0.0556564917235408\end{array}$ $\begin{array}{lllll}0.8892603795187459 & 0.5186608905369586 & 0.2006928033863341\end{array}$ $\begin{array}{lllll}0.3893324928185989 & 0.0186259598562900 & 0.2006885752577619\end{array}$ $\begin{array}{lllll}0.1390945849430147 & 0.7687127973879867 & 0.3451031170853311\end{array}$ $\begin{array}{llll}0.6394146079186991 & 0.2681275190470174 & 0.3451794604279798\end{array}$ $\begin{array}{llll}0.8901356236009552 & 0.5191500806151977 & 0.4905761056692459\end{array}$ $\begin{array}{llll}0.3892148411060752 & 0.0136026610502204 & 0.4898493248539751\end{array}$ $\begin{array}{llll}0.2305390936428786 & 0.8622613350066950 & 0.0729463109990666\end{array}$ $\begin{array}{llll}0.7304846770537643 & 0.3623125686170378 & 0.0729614622621616\end{array}$ $\begin{array}{llll}0.9818308169086022 & 0.6109984275685603 & 0.2177725987230851\end{array}$ $\begin{array}{llll}0.4818749735122426 & 0.1109953679158658 & 0.2177646318954514\end{array}$ $\begin{array}{llll}0.2320593064830695 & 0.8605068216457475 & 0.3623060881716277\end{array}$ $\begin{array}{llll}0.7322929243298515 & 0.3601971659114648 & 0.3622629395917742\end{array}$ $\begin{array}{lllll}0.9839083765181905 & 0.6103524766468784 & 0.5078279301771356\end{array}$ $\begin{array}{llll}0.4838209759161320 & 0.1034442571039737 & 0.5068602419281594\end{array}$ $\begin{array}{llll}0.3286017249450469 & 0.7024887563164951 & 0.0371952486870620\end{array}$ $\begin{array}{llll}0.8286177382522798 & 0.2025108272900480 & 0.0372154355560937\end{array}$ $\begin{array}{llll}0.0799242655876274 & 0.4501668837126173 & 0.1824312965304757\end{array}$ $\begin{array}{llll}0.5799781598175143 & 0.9501110020072903 & 0.1824222978496580\end{array}$ $\begin{array}{llll}0.3300906408933926 & 0.6995275811296413 & 0.3269618299233077\end{array}$ $\begin{array}{llll}0.8302118978210590 & 0.1992011636274698 & 0.3269357150691807\end{array}$ $\begin{array}{llll}0.0806458043095546 & 0.4497259858441240 & 0.4722852396836991\end{array}$ $\begin{array}{llll}0.5819440890656479 & 0.9406390833176567 & 0.4723389481143918\end{array}$ $\begin{array}{llll}0.3006069455439420 & 0.6708444563435744 & 0.0906746370125772\end{array}$ $\begin{array}{llll}0.8005295982496238 & 0.1709013866584294 & 0.0906845893293616\end{array}$ $\begin{array}{lllll}0.0508638457375658 & 0.4199285143528544 & 0.2357768920872053\end{array}$ $\begin{array}{llll}0.5509657391557530 & 0.9199183494409604 & 0.2357725694223272\end{array}$ $\begin{array}{llll}0.3004624018269726 & 0.6695364335815240 & 0.3802699846884727\end{array}$ $\begin{array}{llll}0.8010037472208362 & 0.1693379858623628 & 0.3803116860065905\end{array}$ $\begin{array}{llll}0.0517772928091422 & 0.4196904629438808 & 0.5258232995367519\end{array}$ $\begin{array}{llll}0.5488038902762715 & 0.9148133570382032 & 0.5258812315842123\end{array}$ $\begin{array}{llll}0.2487713111526749 & 0.2504274928617792 & 0.0641074187576949\end{array}$ $\begin{array}{llll}0.7488303185549979 & 0.7503888092593546 & 0.0641033284193118\end{array}$ $\begin{array}{llll}0.0001125482416500 & 0.9995408900696325 & 0.2091237433549188\end{array}$ $\begin{array}{lllll}0.5000093123775916 & 0.4996242974270974 & 0.2091480365575033\end{array}$ $\begin{array}{llll}0.2503916395894490 & 0.2494765516315431 & 0.3536015289005931\end{array}$ $\begin{array}{llll}0.7507859128607733 & 0.7494675411662793 & 0.3535642296631678\end{array}$ $\begin{array}{llll}0.9992481171353397 & 0.9994179796773424 & 0.4996787392900066\end{array}$ $\begin{array}{llll}0.5020116995863351 & 0.4980434947954693 & 0.4980898854281252\end{array}$ $\begin{array}{llll}0.2496615847190023 & 0.7510710282088292 & 0.0641032616106441\end{array}$ $\begin{array}{llll}0.7496238145821522 & 0.2511188626368953 & 0.0641115752959786\end{array}$ $\begin{array}{llll}0.0004878981817987 & 0.4999322750221049 & 0.2091579095980868\end{array}$ $\begin{array}{llll}0.5005646955074606 & 0.9998937120904353 & 0.2091515026053415\end{array}$ $\begin{array}{llll}0.2504950969441503 & 0.7495541152070677 & 0.3536465849877306\end{array}$ $\begin{array}{llll}0.7507669415031551 & 0.2491766333299907 & 0.3536542704958940\end{array}$ $\begin{array}{llll}0.0017881154596183 & 0.4996297348818700 & 0.4991398158902166\end{array}$ $\begin{array}{llll}0.5011045515396577 & 0.9927448286357675 & 0.4987695465688397\end{array}$ $\begin{array}{llll}0.4865910333546730 & 0.4383772920762295 & 0.5998969685378253\end{array}$ $\begin{array}{llll}0.4851577668854614 & 0.5430701399664528 & 0.6073122275153953\end{array}$ 
$\begin{array}{llll}0.5026412062596015 & 0.5576309093639668 & 0.6380097982615431\end{array}$ $\begin{array}{llll}0.5308628548969756 & 0.4764701257546153 & 0.6578802657561144\end{array}$ $\begin{array}{lllll}0.5442893362306076 & 0.3729412353384357 & 0.6501468828059749\end{array}$ $\begin{array}{llll}0.5255801232858095 & 0.3527511524948585 & 0.6227443254442204\end{array}$ $\begin{array}{llll}0.5169138114851247 & 0.1375000979610082 & 0.1046155642502360\end{array}$ $\begin{array}{llll}0.0169761681643615 & 0.6374626412366953 & 0.1046224469853508\end{array}$ $\begin{array}{llll}0.2685646493646534 & 0.8864878046049329 & 0.2493691021283316\end{array}$ $\begin{array}{llll}0.7684535238453271 & 0.3865122334467638 & 0.2494206939816833\end{array}$ $\begin{array}{llll}0.5195946386823254 & 0.1340102440037800 & 0.3938732156845697\end{array}$ $\begin{array}{llll}0.0190306011910849 & 0.6364739622301178 & 0.3939772991336362\end{array}$ $\begin{array}{llll}0.2696989822534441 & 0.8822037242154922 & 0.5402220339449730\end{array}$ $\begin{array}{llll}0.7695774449215966 & 0.3808983046925773 & 0.5394645980307657\end{array}$ $\begin{array}{llll}0.3638562506575064 & 0.9819813323199960 & 0.0231714528738322\end{array}$ $\begin{array}{llll}0.8638676813089582 & 0.4819612025070180 & 0.0231685300545464\end{array}$ $\begin{array}{llll}0.1132593099233172 & 0.7316693549107277 & 0.1686227516591546\end{array}$ $\begin{array}{llll}0.6132278998561660 & 0.2317037312691109 & 0.1686515894713389\end{array}$ $\begin{array}{llll}0.3637618053248188 & 0.9813304182162670 & 0.3132367453520151\end{array}$ $\begin{array}{llll}0.8637886801021583 & 0.4812971660633744 & 0.3132698845372465\end{array}$ $\begin{array}{lllll}0.1131963057477151 & 0.7322755219009176 & 0.4584155587109816\end{array}$ $\begin{array}{lllll}0.6133374070660218 & 0.2301153884798911 & 0.4577767982905597\end{array}$ $\begin{array}{llll}0.1312742393188031 & 0.5099587729288204 & 0.0186340777190246\end{array}$ $\begin{array}{lllll}0.6312187805595384 & 0.0099933806243746 & 0.0186576462186469\end{array}$ $\begin{array}{llll}0.8795047068343328 & 0.2601073501376475 & 0.1644573746969323\end{array}$ $\begin{array}{llll}0.3794863161877500 & 0.7601342298222680 & 0.1644589627808921\end{array}$ $\begin{array}{llll}0.1295054616359194 & 0.5100922374496036 & 0.3088644180058496\end{array}$ $\begin{array}{llll}0.6296801999129341 & 0.0100899806309662 & 0.3088332529486695\end{array}$ $\begin{array}{lllll}0.8820059717247003 & 0.2578623209324097 & 0.4540478819561134\end{array}$ $\begin{array}{llll}0.3807589656520250 & 0.7585449000670494 & 0.4534657225878522\end{array}$ $\begin{array}{llll}0.9899779002506648 & 0.3705750668715471 & 0.1096325532311180\end{array}$ $\begin{array}{llll}0.4899943798802651 & 0.8704908438239751 & 0.1096014214563186\end{array}$ $\begin{array}{llll}0.7396967386915123 & 0.1203521275977766 & 0.2539050001215853\end{array}$ $\begin{array}{llll}0.2396916707461542 & 0.6201926400074723 & 0.2539612910083880\end{array}$ $\begin{array}{llll}0.9909720476287468 & 0.3698411200661207 & 0.3987398351524825\end{array}$ $\begin{array}{llll}0.4903419372319129 & 0.8695669356102396 & 0.3983593198459102\end{array}$ $\begin{array}{llll}0.7454024127691170 & 0.1087486348307409 & 0.5469937197804623\end{array}$ $\begin{array}{llll}0.2467327888805528 & 0.6177269181225933 & 0.5444683744676451\end{array}$ $\begin{array}{llll}0.9900626669512489 & 0.8687346443102970 & 0.0186421764526434\end{array}$ $\begin{array}{llll}0.4901180909203333 & 0.3688675751610798 & 0.0186037958235184\end{array}$ $\begin{array}{llll}0.7399117495044213 & 0.6206051263462702 & 0.1644942059175826\end{array}$ $\begin{array}{llll}0.2399655612613402 & 0.1206055850111486 & 0.1644946031121601\end{array}$ $\begin{array}{llll}0.9900954172099578 & 0.8703884858257265 & 0.3088446062867433\end{array}$ $\begin{array}{llll}0.4901249900334484 & 0.3701686256609776 & 0.3089474995979659\end{array}$ $\begin{array}{llll}0.7438904702814958 & 0.6176193108241501 & 0.4534321604088989\end{array}$ $\begin{array}{llll}0.2407193248709364 & 0.1188189244496626 & 0.4535120509251999\end{array}$ $\begin{array}{llll}0.1295604691199034 & 0.0100394479249092 & 0.1095858504631859\end{array}$ $\begin{array}{llll}0.6295475168831290 & 0.5100319246925282 & 0.1096079263321710\end{array}$ $\begin{array}{llll}0.8797524168388020 & 0.7603375139453515 & 0.2539267951484005\end{array}$ $\begin{array}{llll}0.3796696204964834 & 0.2603878255904598 & 0.2539040828755363\end{array}$ $\begin{array}{llll}0.1297601272963336 & 0.0094683445949424 & 0.3985987030581753\end{array}$ $\begin{array}{llll}0.6305462437085065 & 0.5094880232914787 & 0.3984444470924723\end{array}$ $\begin{array}{llll}0.8863456346060098 & 0.7567699433215175 & 0.5454154749781355\end{array}$ $\begin{array}{lllll}0.3879155635330401 & 0.2551258827683347 & 0.5426719960886579\end{array}$ $\begin{array}{llll}0.5181697092692802 & 0.6360372224902927 & 0.0231802530809354\end{array}$ $\begin{array}{lllll}0.0181764863542810 & 0.1360203381979119 & 0.0231957958521654\end{array}$ $\begin{array}{llll}0.2683577714063897 & 0.3867050489555677 & 0.1686416678846961\end{array}$ $\begin{array}{llll}0.7683885571346628 & 0.8866824953551803 & 0.1686151352208575\end{array}$ 
$\begin{array}{llll}0.5185875433638059 & 0.6362129957423164 & 0.3132619260593744\end{array}$ $\begin{array}{llll}0.0186269785282890 & 0.1360977196358956 & 0.3131914423414511\end{array}$ $\begin{array}{llll}0.2687489196629561 & 0.3848225745576108 & 0.4576721719590784\end{array}$ $\begin{array}{llll}0.7681377448900562 & 0.8841365014838752 & 0.4583614817949531\end{array}$ $\begin{array}{llll}0.3624493412396436 & 0.4829545150631813 & 0.1046225874898226\end{array}$ $\begin{array}{llll}0.8624183040875514 & 0.9829858647722951 & 0.1046222255147627\end{array}$ $\begin{array}{llll}0.1134496569009225 & 0.2314458403139793 & 0.2493537936643691\end{array}$ $\begin{array}{llll}0.6135151387416166 & 0.7314616582916494 & 0.2493817154410824\end{array}$ $\begin{array}{llll}0.3634234810855327 & 0.4809843453070368 & 0.3938690006157322\end{array}$ $\begin{array}{llll}0.8639001066938866 & 0.9810054964801294 & 0.3941265297473056\end{array}$ $\begin{array}{llll}0.1165741656571702 & 0.2305077667588654 & 0.5397205286093896\end{array}$ $\begin{array}{llll}0.6174474362984289 & 0.7252615884318843 & 0.5408012581852680\end{array}$ $\begin{array}{llll}0.4400001844071013 & 0.1628825857336020 & 0.0805282556248853\end{array}$ $\begin{array}{llll}0.9400882863937430 & 0.6627695550376224 & 0.0805290455643695\end{array}$ $\begin{array}{lllll}0.1910298292854729 & 0.9125332539498301 & 0.2254529676226617\end{array}$ $\begin{array}{llll}0.6908948606543586 & 0.4127105811991418 & 0.2254986929933167\end{array}$ $\begin{array}{llll}0.4412462772761102 & 0.1629944404864087 & 0.3699132355497315\end{array}$ $\begin{array}{llll}0.9417394730424095 & 0.6626874698338240 & 0.3699510751453456\end{array}$ $\begin{array}{llll}0.1911343219363032 & 0.9096830292016922 & 0.5160487911032472\end{array}$ $\begin{array}{llll}0.6917685492980684 & 0.4142327391696821 & 0.5152505054443033\end{array}$ $\begin{array}{llll}0.3353663945272251 & 0.0601424116999659 & 0.0472862288129477\end{array}$ $\begin{array}{lllll}0.8353735358046027 & 0.5601277634000267 & 0.0472816300434002\end{array}$ $\begin{array}{llll}0.0866106460730684 & 0.8089279406147333 & 0.1925831827836265\end{array}$ $\begin{array}{llll}0.5866050012044284 & 0.3089533847104501 & 0.1926154732352054\end{array}$ $\begin{array}{llll}0.3369730526841270 & 0.0588054154175054 & 0.3371432732269072\end{array}$ $\begin{array}{llll}0.8373798797222178 & 0.5586928161410196 & 0.3371693469144685\end{array}$ $\begin{array}{llll}0.0855086064893360 & 0.8089403456059788 & 0.4826774904606318\end{array}$ $\begin{array}{llll}0.5877899660135807 & 0.3073251370324235 & 0.4816361117406174\end{array}$ $\begin{array}{llll}0.1424313386874038 & 0.4148158007498333 & 0.0332304094461266\end{array}$ $\begin{array}{llll}0.6424280226291149 & 0.9148009812370242 & 0.0332350107125772\end{array}$ $\begin{array}{llll}0.8899528328961416 & 0.1667247812191373 & 0.1791394404271717\end{array}$ $\begin{array}{llll}0.3898771135440273 & 0.6667918989347116 & 0.1791500960247445\end{array}$ $\begin{array}{llll}0.1400601861495644 & 0.4165483489223467 & 0.3235103726239435\end{array}$ $\begin{array}{llll}0.6404696144511340 & 0.9164139063742422 & 0.3234659611210706\end{array}$ $\begin{array}{llll}0.8909635930830935 & 0.1652939595999351 & 0.4690106735756435\end{array}$ $\begin{array}{llll}0.3921647497031344 & 0.6650595681563363 & 0.4681334406961698\end{array}$ $\begin{array}{llll}0.0831311791527461 & 0.3602025198595689 & 0.0946653310090676\end{array}$ $\begin{array}{llll}0.5831685487748283 & 0.8601121881577301 & 0.0946435466599652\end{array}$ $\begin{array}{lllll}0.8332077267987893 & 0.1098487125554375 & 0.2391762920006835\end{array}$ $\begin{array}{llll}0.3331237093007142 & 0.6098273515635528 & 0.2392185988756442\end{array}$ $\begin{array}{llll}0.0840068995617561 & 0.3594309057218082 & 0.3837901503207015\end{array}$ $\begin{array}{llll}0.5839504932637624 & 0.8592893610759622 & 0.3837083788528123\end{array}$ $\begin{array}{llll}0.8382335184708868 & 0.1011017041531420 & 0.5320947613357302\end{array}$ $\begin{array}{llll}0.3316381643093975 & 0.6133622706836928 & 0.5268484467127806\end{array}$ $\begin{array}{lllll}0.0852529503762723 & 0.8575183178171587 & 0.0332322564036421\end{array}$ $\begin{array}{llll}0.5853542344347760 & 0.3575444983905503 & 0.0332008819767612\end{array}$ $\begin{array}{llll}0.8332347796716826 & 0.6101857416632848 & 0.1792042678869864\end{array}$ $\begin{array}{llll}0.3333480248739751 & 0.1101238564697562 & 0.1791900516026060\end{array}$ $\begin{array}{llll}0.0835237918952954 & 0.8599185059807353 & 0.3235200863110144\end{array}$ $\begin{array}{llll}0.5836237050533769 & 0.3595173305833705 & 0.3236474286382017\end{array}$ $\begin{array}{llll}0.8356896400565618 & 0.6090640773831094 & 0.4686533570472525\end{array}$ $\begin{array}{lllll}0.3353887561508313 & 0.1052853579101496 & 0.4683760923206944\end{array}$ $\begin{array}{llll}0.1399486397068927 & 0.9168132411952560 & 0.0946488878845094\end{array}$ $\begin{array}{llll}0.6398961267191834 & 0.4168312239092569 & 0.0946672029766376\end{array}$ $\begin{array}{llll}0.8902518518167414 & 0.6668148174582393 & 0.2392165436773837\end{array}$ $\begin{array}{llll}0.3902547473193353 & 0.1668094156850131 & 0.2391942517183538\end{array}$ 
$\begin{array}{llll}0.1403943243779075 & 0.9161909398035296 & 0.3837255344058731\end{array}$ $\begin{array}{llll}0.6412776857743838 & 0.4156353142293169 & 0.3838603868947408\end{array}$ $\begin{array}{lllll}0.8966689219234831 & 0.6628111562049727 & 0.5304576143910930\end{array}$ $\begin{array}{llll}0.3991793067114351 & 0.1563391213778954 & 0.5299947346648356\end{array}$ $\begin{array}{llll}0.4399543371533350 & 0.6645660679105228 & 0.0472876500345202\end{array}$ $\begin{array}{llll}0.9399525472419291 & 0.1646382791076734 & 0.0473094374531289\end{array}$ $\begin{array}{llll}0.1910978202349297 & 0.4133967053484848 & 0.1926107537741960\end{array}$ $\begin{array}{llll}0.6911496323500401 & 0.9133652061010652 & 0.1925886541277347\end{array}$ $\begin{array}{llll}0.4412335045145060 & 0.6627494635297240 & 0.3371831349237627\end{array}$ $\begin{array}{llll}0.9414038698418942 & 0.1623664458871449 & 0.3371248194717989\end{array}$ $\begin{array}{llll}0.1925876670170726 & 0.4113803187964646 & 0.4820662133513709\end{array}$ $\begin{array}{lllll}0.6924364135299734 & 0.9059231799799111 & 0.4824164657019830\end{array}$ $\begin{array}{lllll}0.3370737128836007 & 0.5598419859494971 & 0.0805255572208724\end{array}$ $\begin{array}{llll}0.8369755603512148 & 0.0599148970743861 & 0.0805329606265453\end{array}$ $\begin{array}{llll}0.0873139478332675 & 0.3090448250625247 & 0.2254563384658735\end{array}$ $\begin{array}{llll}0.5873896307602290 & 0.8090248760301673 & 0.2254762616633712\end{array}$ $\begin{array}{llll}0.3372223722801115 & 0.5585784245496948 & 0.3699377610439506\end{array}$ $\begin{array}{llll}0.8378245926649557 & 0.0582507653521609 & 0.3700952589592229\end{array}$ $\begin{array}{llll}0.0890261909795622 & 0.3086125286433358 & 0.5156480834542138\end{array}$ $\begin{array}{llll}0.5873252748123237 & 0.8028660947427845 & 0.5162184586430963\end{array}$ $\begin{array}{llll}0.6105201375768045 & 0.1033064151142390 & 0.0958509701871211\end{array}$ $\begin{array}{llll}0.1105864252591114 & 0.6032702000894213 & 0.0958631156657442\end{array}$ $\begin{array}{lllll}0.3620810157007758 & 0.8520019177652247 & 0.2404341934740778\end{array}$ $\begin{array}{llll}0.8619500246522052 & 0.3521094993098427 & 0.2404855280081948\end{array}$ $\begin{array}{llll}0.6128896586803029 & 0.1003509924814884 & 0.3849486795631476\end{array}$ $\begin{array}{llll}0.1127227238166896 & 0.6018019418757184 & 0.3851497370275654\end{array}$ $\begin{array}{llll}0.3629623220968617 & 0.8478937601226870 & 0.5310533978072525\end{array}$ $\begin{array}{llll}0.8618274315407657 & 0.3533226041262056 & 0.5311447707826205\end{array}$ $\begin{array}{llll}0.3967589048325714 & 0.8888495742784863 & 0.0322014786813685\end{array}$ $\begin{array}{llll}0.8967328242837340 & 0.3888428116100593 & 0.0322006638980509\end{array}$ $\begin{array}{llll}0.1479453485143336 & 0.6379183589127565 & 0.1774190371649181\end{array}$ $\begin{array}{llll}0.6479143668602115 & 0.1379557083788129 & 0.1774490643534276\end{array}$ $\begin{array}{llll}0.3986505387592084 & 0.8875942314890479 & 0.3220867739980590\end{array}$ $\begin{array}{llll}0.8985125415836644 & 0.3876180273758780 & 0.3221224835411042\end{array}$ $\begin{array}{llll}0.1476444380779920 & 0.6382580071860398 & 0.4668668327982234\end{array}$ $\begin{array}{llll}0.6481142993191097 & 0.1364392408958354 & 0.4666619194300395\end{array}$ $\begin{array}{lllll}0.0528971854427621 & 0.5579449742016038 & 0.0014512933109172\end{array}$ $\begin{array}{llll}0.5527770041339833 & 0.0580489681566235 & 0.0014946234842749\end{array}$ $\begin{array}{llll}0.7999879455476240 & 0.3088835087803132 & 0.1474378967312402\end{array}$ $\begin{array}{llll}0.3000010803974969 & 0.8088953725099844 & 0.1474293140169208\end{array}$ $\begin{array}{llll}0.0499509651955032 & 0.5589230489157779 & 0.2918580394856728\end{array}$ $\begin{array}{lllll}0.5500089432110022 & 0.0589343958696830 & 0.2918644704786057\end{array}$ $\begin{array}{lllll}0.8039911639353376 & 0.3054919512814919 & 0.4365997139795335\end{array}$ $\begin{array}{llll}0.3001971979705036 & 0.8079411239832425 & 0.4367526993550899\end{array}$ $\begin{array}{llll}0.9422516335470348 & 0.4495394067109511 & 0.1267901429651905\end{array}$ $\begin{array}{llll}0.4421984454513707 & 0.9495192427250048 & 0.1267380573294890\end{array}$ $\begin{array}{llll}0.6907881592934839 & 0.2000363229290918 & 0.2708406298073771\end{array}$ $\begin{array}{lllll}0.1907870137703513 & 0.6998407280923077 & 0.2709064862070211\end{array}$ $\begin{array}{llll}0.9432637986163953 & 0.4488249619922812 & 0.4159057094019352\end{array}$ $\begin{array}{llll}0.4414766746273386 & 0.9489335157475348 & 0.4153855182842207\end{array}$ $\begin{array}{lllll}0.7024620652479305 & 0.1804233777702377 & 0.5660587053372068\end{array}$ $\begin{array}{lllll}0.2037647010966615 & 0.6936754315966801 & 0.5624416865182208\end{array}$ $\begin{array}{llll}0.9420930869882924 & 0.9471064138136972 & 0.0014589393934637\end{array}$ $\begin{array}{llll}0.4423013526109110 & 0.4471609229935590 & 0.0013965078141460\end{array}$ $\begin{array}{llll}0.6910427601675994 & 0.7002700116651485 & 0.1475201638684858\end{array}$ $\begin{array}{llll}0.1911303210471002 & 0.2002498192178751 & 0.1475136150872681\end{array}$ 
$\begin{array}{llll}0.9413142225064257 & 0.9498846117869265 & 0.2918203619810649\end{array}$ $\begin{array}{llll}0.4412632544897835 & 0.4499209679410008 & 0.2920009948756245\end{array}$ $\begin{array}{llll}0.6965977324322777 & 0.6956204835204179 & 0.4359622694276678\end{array}$ $\begin{array}{llll}0.1935637507060364 & 0.1989150789053091 & 0.4366649831927347\end{array}$ $\begin{array}{llll}0.0505205696128329 & 0.0578747615429132 & 0.1267182205534251\end{array}$ $\begin{array}{llll}0.5505094103865051 & 0.5578974132462928 & 0.1267407489411155\end{array}$ $\begin{array}{llll}0.8000398651717863 & 0.8093029343971420 & 0.2708545938785375\end{array}$ $\begin{array}{lllll}0.2999801032902131 & 0.3092772889532186 & 0.2708392627897222\end{array}$ $\begin{array}{llll}0.0503598009192798 & 0.0576486579299059 & 0.4156282038667219\end{array}$ $\begin{array}{llll}0.5510376589244992 & 0.5583221310329649 & 0.4154331806600796\end{array}$ $\begin{array}{llll}0.8085550635362373 & 0.8038963494510450 & 0.5627992926206430\end{array}$ $\begin{array}{llll}0.3133029705768098 & 0.3033463170983586 & 0.5609038986786111\end{array}$ $\begin{array}{llll}0.6112366337755609 & 0.6032960872592319 & 0.0322217463986582\end{array}$ $\begin{array}{llll}0.1112473462221974 & 0.1033055765542753 & 0.0322275512383011\end{array}$ $\begin{array}{llll}0.3621416299456073 & 0.3519359712794535 & 0.1774303373996543\end{array}$ $\begin{array}{lllll}0.8621484215874644 & 0.8519991365653626 & 0.1774022784975998\end{array}$ $\begin{array}{llll}0.6123358782284816 & 0.6013635664732657 & 0.3220933195511593\end{array}$ $\begin{array}{llll}0.1123753898125726 & 0.1013027367319082 & 0.3220096465724814\end{array}$ $\begin{array}{llll}0.3634416242147167 & 0.3480958200096075 & 0.4660307041165667\end{array}$ $\begin{array}{llll}0.8610405187095355 & 0.8521495140709696 & 0.4671495434851915\end{array}$ $\begin{array}{lllll}0.3966744601718806 & 0.3893293313574782 & 0.0958674422381744\end{array}$ $\begin{array}{llll}0.8966529942405230 & 0.8893654910488689 & 0.0958596108438808\end{array}$ $\begin{array}{lllll}0.1480043275982098 & 0.1379189523239917 & 0.2404080749211115\end{array}$ $\begin{array}{lllll}0.6478979705386489 & 0.6379836780388872 & 0.2404345316380088\end{array}$ $\begin{array}{lllll}0.3978300147935326 & 0.3874777431772787 & 0.3849398316345921\end{array}$ $\begin{array}{llll}0.8984151026708114 & 0.8873402365336168 & 0.3853000027429228\end{array}$ $\begin{array}{llll}0.1489578891309988 & 0.1375789431646608 & 0.5306890746492350\end{array}$ $\begin{array}{llll}0.6518307494967308 & 0.6313192328278490 & 0.5321963177505457\end{array}$ $\begin{array}{llll}0.4802989203014141 & 0.1527297766654082 & 0.1294158103055011\end{array}$ $\begin{array}{llll}0.9803328558024678 & 0.6527426605100395 & 0.1294205211322033\end{array}$ $\begin{array}{llll}0.2326272476599305 & 0.9015820701346144 & 0.2742455111690585\end{array}$ $\begin{array}{llll}0.7325567994298486 & 0.4013986387910984 & 0.2742979318156899\end{array}$ $\begin{array}{lllll}0.4844100958506503 & 0.1461931966806979 & 0.4187382823975986\end{array}$ $\begin{array}{llll}0.9826952793123177 & 0.6516379836630818 & 0.4188083320297756\end{array}$ $\begin{array}{llll}0.2342745250177490 & 0.8967265969798203 & 0.5650249702624570\end{array}$ $\begin{array}{lllll}0.7364015743649911 & 0.3833647366346598 & 0.5640379341061631\end{array}$ $\begin{array}{llll}0.3521818950685816 & 0.0167754229639568 & 0.9983633105461325\end{array}$ $\begin{array}{llll}0.8522423376627779 & 0.5167277258813299 & 0.9983586149737894\end{array}$ $\begin{array}{llll}0.0984380414664279 & 0.7678540975903457 & 0.1437607828997334\end{array}$ $\begin{array}{lllll}0.5983700720463070 & 0.2679012629560714 & 0.1437903214967349\end{array}$ $\begin{array}{llll}0.3488262791956293 & 0.0173688403011971 & 0.2883535551447425\end{array}$ $\begin{array}{llll}0.8487685182098835 & 0.5173316610751870 & 0.2883846317239181\end{array}$ $\begin{array}{llll}0.0992614220755626 & 0.7693634020973876 & 0.4336791201959810\end{array}$ $\begin{array}{lllll}0.5977884835505977 & 0.2661240845016841 & 0.4328632651096116\end{array}$ $\begin{array}{llll}0.1994190071151364 & 0.5279007120563541 & 0.0252457528840009\end{array}$ $\begin{array}{llll}0.6993911395607012 & 0.0279049309964604 & 0.0252637220304412\end{array}$ $\begin{array}{llll}0.9481379464219895 & 0.2778037411335290 & 0.1705221666433610\end{array}$ $\begin{array}{llll}0.4481266512193836 & 0.7778145292005417 & 0.1705286278157730\end{array}$ $\begin{array}{llll}0.1980944695564366 & 0.5278231716498295 & 0.3149480910644687\end{array}$ $\begin{array}{llll}0.6982482459470729 & 0.0278798707793444 & 0.3148907819511055\end{array}$ $\begin{array}{llll}0.9502146177507877 & 0.2757605371514510 & 0.4604411257775953\end{array}$ $\begin{array}{llll}0.4497638667766590 & 0.7761956175957547 & 0.4591778051867389\end{array}$ $\begin{array}{llll}0.9716959520018541 & 0.3024218698355388 & 0.1035134177413760\end{array}$ $\begin{array}{llll}0.4717584414331744 & 0.8022920601083428 & 0.1034980193417106\end{array}$ $\begin{array}{llll}0.7222147521834035 & 0.0515430029497189 & 0.2478703427163548\end{array}$ $0.2222601414667454 \quad 0.5513491571723904 \quad 0.2479227813242032$ 
$\begin{array}{llll}0.9726111380935610 & 0.3017209041164211 & 0.3926525406628373\end{array}$ $\begin{array}{lllll}0.4728201176595736 & 0.8009138280815624 & 0.3921608817860830\end{array}$ $\begin{array}{lllll}0.7238836060853732 & 0.0447847736679887 & 0.5387779358107720\end{array}$ $\begin{array}{llll}0.2285093956526750 & 0.5487526808161676 & 0.5392021052860756\end{array}$ $\begin{array}{llll}0.9720716078205104 & 0.8006357995267559 & 0.0252630298302900\end{array}$ $\begin{array}{llll}0.4719282991610379 & 0.3009345686827405 & 0.0252442154845787\end{array}$ $\begin{array}{llll}0.7223442171274096 & 0.5518275910233535 & 0.1704930560823668\end{array}$ $\begin{array}{lllll}0.2223238316060306 & 0.0518929422857601 & 0.1705096690228628\end{array}$ $\begin{array}{llll}0.9723852201611386 & 0.8017692898529192 & 0.3149189266400583\end{array}$ $\begin{array}{llll}0.4724577548082033 & 0.3014616272934512 & 0.3149270094063032\end{array}$ $\begin{array}{llll}0.7260849225537992 & 0.5492223310489031 & 0.4596597105884235\end{array}$ $\begin{array}{llll}0.2205926567166581 & 0.0518289525302500 & 0.4593131339716297\end{array}$ $\begin{array}{llll}0.1977628872611766 & 0.0282614111242996 & 0.1034766774766564\end{array}$ $\begin{array}{llll}0.6977709689075915 & 0.5282227705340148 & 0.1034948966282855\end{array}$ $\begin{array}{llll}0.9485765091777363 & 0.7777933188266442 & 0.2478873309208810\end{array}$ $\begin{array}{lllll}0.4484199565505072 & 0.2779362286988927 & 0.2478574676406728\end{array}$ $\begin{array}{llll}0.1981656824228538 & 0.0275219565076804 & 0.3925668503546220\end{array}$ $\begin{array}{llll}0.6990491403703328 & 0.5272386256401523 & 0.3922645117312240\end{array}$ $\begin{array}{llll}0.9543956332508290 & 0.7749816250906452 & 0.5389034342683178\end{array}$ $\begin{array}{llll}0.4525858183475978 & 0.2739967824752426 & 0.5334989519498932\end{array}$ $\begin{array}{llll}0.4834790044048481 & 0.6475239309711776 & 0.9983679000182978\end{array}$ $\begin{array}{llll}0.9834875645092305 & 0.1474261395303621 & 0.9983864881544749\end{array}$ $\begin{array}{llll}0.2321199388639776 & 0.4015973413781259 & 0.1437842356862762\end{array}$ $\begin{array}{llll}0.7321677462401629 & 0.9014885920436461 & 0.1437578437231756\end{array}$ $\begin{array}{lllll}0.4824734619307331 & 0.6512673321352817 & 0.2883839341785824\end{array}$ $\begin{array}{llll}0.9824086434708392 & 0.1513078291832718 & 0.2883206221559109\end{array}$ $\begin{array}{llll}0.2303513194103880 & 0.4017765749325264 & 0.4330081446242249\end{array}$ $\begin{array}{llll}0.7315591805372710 & 0.8996270685170135 & 0.4335428637404530\end{array}$ $\begin{array}{llll}0.3471976971191943 & 0.5196165111934796 & 0.1294190312907265\end{array}$ $\begin{array}{llll}0.8471902088172303 & 0.0196112351275618 & 0.1294222614673914\end{array}$ $\begin{array}{llll}0.0983575487075813 & 0.2673375358699194 & 0.2742357850985079\end{array}$ $\begin{array}{llll}0.5986002115505791 & 0.7673200526678204 & 0.2742635218525270\end{array}$ $\begin{array}{lllll}0.3485084897250336 & 0.5169126269705649 & 0.4187387784660054\end{array}$ $\begin{array}{llll}0.8488073854686015 & 0.0173278088746738 & 0.4189514971365811\end{array}$ $\begin{array}{llll}0.1047262761657973 & 0.2652630125463772 & 0.5645515973435937\end{array}$ $\begin{array}{llll}0.6056387931870113 & 0.7617054875445816 & 0.5654124544646830\end{array}$ $\begin{array}{lllll}0.4529734744825072 & 0.4325091413865035 & 0.5758691044520351\end{array}$ $\begin{array}{llll}0.4710158283436313 & 0.6071898039302717 & 0.5876211272533676\end{array}$ 

BQ molecule in triplet $\mathrm{T1}^{3}\left(\mathrm{n}, \pi^{*}\right)$ state on PETN (110) surface
Method: optPBE-vdW

BQ-PETN_T1

1.00000000000000

$\begin{array}{llll}14.7659997940000007 & 0.0000000000000000 & 0.0000000000000000\end{array}$

$\begin{array}{llll}8.8218669359999993 & 11.8410056025999992 & 0.0000000000000000\end{array}$

$\begin{array}{llll}0.0000000000000000 & 0.0000000000000000 & 45.7868003844999976\end{array}$

$\begin{array}{llll}\mathrm{H} & \mathrm{C} & \mathrm{N} & \mathrm{O}\end{array}$

$\begin{array}{llll}132 & 86 & 64 & 194\end{array}$

Direct

$\begin{array}{llll}0.3365679159459615 & 0.1257674057533798 & 0.0991995041415660\end{array}$

$\begin{array}{lllll}0.8366094902864449 & 0.6256853792139772 & 0.0991861551008659\end{array}$

$\begin{array}{lllll}0.0876082326484669 & 0.8757279712218813 & 0.2443593561507380\end{array}$

$\begin{array}{lllll}0.5875500845015051 & 0.3758175235192774 & 0.2443856108469646\end{array}$

$\begin{array}{lllll}0.33815868866844064 & 0.1256038156983668 & 0.3888923291808334\end{array}$

$\begin{array}{llllll}0.8384970873700704 & 0.6256962074931031 & 0.3889045555450384\end{array}$

$\begin{array}{lllll}0.0845471535343508 & 0.8770406106918658 & 0.5357407260838242\end{array}$

$\begin{array}{lllll}0.5893472440273304 & 0.3732031571082743 & 0.5333180652531230\end{array}$

$\begin{array}{lllll}0.3693561160098222 & 0.1653058930023332 & 0.0278414700997840\end{array}$

$\begin{array}{llllll}0.8693535774914807 & 0.6652656632567768 & 0.0278369127497144\end{array}$

$\begin{array}{lllll}0.1230298581713276 & 0.9125915632326774 & 0.1735965633566164\end{array}$

$\begin{array}{lllll}0.6229957906025693 & 0.4125885923489822 & 0.1736172754058561\end{array}$

$\begin{array}{lllll}0.3733915524223611 & 0.1623846376560217 & 0.3181224144129199\end{array}$

$\begin{array}{lllll}0.8741274440474541 & 0.6620750985158406 & 0.3181069550895006\end{array}$

$\begin{array}{llllll}0.1210940994264446 & 0.9137511393984790 & 0.4642623023203082\end{array}$

$\begin{array}{lllll}0.6258759223155139 & 0.4090734977427719 & 0.4629896234558962\end{array}$

$\begin{array}{lllll}0.2011611684469641 & 0.4184021361065802 & 0.0744161365772776\end{array}$

$\begin{array}{lllll}0.7012491169897141 & 0.9183490770304705 & 0.0744139506521277\end{array}$

$\begin{array}{lllll}0.9554521742559396 & 0.1665614288473830 & 0.2195180451127516\end{array}$

$\begin{array}{llllll}0.4553626358684947 & 0.6666170584149468 & 0.2195134481493697\end{array}$

$\begin{array}{lllll}0.2055933401010997 & 0.4164808504944009 & 0.3640080327487475\end{array}$

$\begin{array}{lllll}0.7065774432073119 & 0.9160854709858353 & 0.3639438826315162\end{array}$

$\begin{array}{lllll}0.9533239608391331 & 0.1673550431432257 & 0.5101588533671633\end{array}$

$\begin{array}{lllll}0.4596077672087620 & 0.6640392164140159 & 0.5083745457592014\end{array}$ $\begin{array}{lllll}0.0808636292526731 & 0.2960220641550240 & 0.0541413008368739\end{array}$ $\begin{array}{llllll}0.5808704008481774 & 0.7960200024987520 & 0.0541378917044804\end{array}$ $\begin{array}{lllll}0.8329918102385577 & 0.0439671252383999 & 0.1987807837226926\end{array}$ $\begin{array}{lllll}0.3329064155506982 & 0.5440445263015834 & 0.1987705170364705\end{array}$ $\begin{array}{lllll}0.0831142654252975 & 0.2939644055343856 & 0.3434337392664512\end{array}$ $\begin{array}{lllll}0.5839698383935951 & 0.7933919898624143 & 0.3432580583547949\end{array}$ $\begin{array}{lllll}0.8285316213518432 & 0.0519661631247390 & 0.4901658930724838\end{array}$ $\begin{array}{lllll}0.3346825417501307 & 0.5431565785526321 & 0.4875077151563252\end{array}$ $\begin{array}{lllll}0.0816783491312232 & 0.7986856502407325 & 0.0744189176948786\end{array}$ $\begin{array}{lllll}0.5816866727343399 & 0.2987051563960926 & 0.0744207206894741\end{array}$ $\begin{array}{lllll}0.8334670445243655 & 0.5444835408329467 & 0.2195475562004133\end{array}$ $\begin{array}{lllll}0.3336139086035190 & 0.0443545012050919 & 0.2195726230275784\end{array}$ $\begin{array}{lllll}0.0833399111092420 & 0.7946242143462519 & 0.3640301970690860\end{array}$ $\begin{array}{lllll}0.5838308345973177 & 0.2938746514807506 & 0.3641547082019636\end{array}$ $\begin{array}{lllll}0.8336008933303580 & 0.5460222425023639 & 0.5094552344454858\end{array}$ $\begin{array}{lllll}0.3327346167154575 & 0.0402266015075633 & 0.5088452134346818\end{array}$ $\begin{array}{lllll}0.2042819175214479 & 0.9188855921863037 & 0.0541467070173785\end{array}$ $\begin{array}{lllll}0.7042192496493843 & 0.4189544855231473 & 0.0541634047602584\end{array}$ $\begin{array}{lllll}0.9561120923909326 & 0.6670185362587869 & 0.1987907684100496\end{array}$ $\begin{array}{lllll}0.4561619996351112 & 0.1669745318306567 & 0.1987879556665624\end{array}$ $\begin{array}{lllll}0.2063116862482245 & 0.9164849535741194 & 0.3433958801272146\end{array}$ $\begin{array}{lllll}0.7063694886499952 & 0.4161853621608944 & 0.3433284049575129\end{array}$ 
$\begin{array}{llll}0.9536701836714668 & 0.6686210511086863 & 0.4896329400607430\end{array}$ $\begin{array}{lllll}0.4539429387069235 & 0.1603216006230377 & 0.4879829812104595\end{array}$ $\begin{array}{llll}0.3348951674188680 & 0.6303956527900637 & 0.0278819857861068\end{array}$ $\begin{array}{llll}0.8350432964311578 & 0.1303825520035996 & 0.0279194876848750\end{array}$ $\begin{array}{llll}0.0873476971334225 & 0.3770576795953821 & 0.1735945679425361\end{array}$ $\begin{array}{llll}0.5874051046584698 & 0.8770396442911501 & 0.1736073998850233\end{array}$ $\begin{array}{llll}0.3376056731990845 & 0.6262192342340934 & 0.3181955887568946\end{array}$ $\begin{array}{llll}0.8377484054896041 & 0.1257038471157861 & 0.3182411750029339\end{array}$ $\begin{array}{llll}0.0870972945180334 & 0.3778501912040463 & 0.4636696269205571\end{array}$ $\begin{array}{llll}0.5901298265019165 & 0.8662563714866170 & 0.4642269770892533\end{array}$ $\begin{array}{lllll}0.3743082247406664 & 0.6631816716789682 & 0.0992025106853352\end{array}$ $\begin{array}{llll}0.8741704329111822 & 0.1633425407612946 & 0.0992597038619178\end{array}$ $\begin{array}{llll}0.1243152521362882 & 0.4123045609450158 & 0.2443489119375246\end{array}$ $\begin{array}{lllll}0.6243252657108764 & 0.9123839110483417 & 0.2443800566418776\end{array}$ $\begin{array}{llll}0.3739895097071848 & 0.6619719969722750 & 0.3890423429739599\end{array}$ $\begin{array}{llll}0.8744193356924754 & 0.1619302370865422 & 0.3890406474347129\end{array}$ $\begin{array}{llll}0.1244569713046356 & 0.4126830840807757 & 0.5350672801608115\end{array}$ $\begin{array}{lllll}0.6208798337150165 & 0.9090183603661867 & 0.5352435063325165\end{array}$ $\begin{array}{llll}0.2994100092043136 & 0.2623908303313446 & 0.1078419597193613\end{array}$ $\begin{array}{llll}0.7994274615987919 & 0.7623080198066573 & 0.1078492249932261\end{array}$ $\begin{array}{llll}0.0503549094928200 & 0.0124743995420717 & 0.2529166774414822\end{array}$ $\begin{array}{llll}0.5501637842231815 & 0.5126320756944055 & 0.2529328224556869\end{array}$ $\begin{array}{llll}0.3006533904179682 & 0.2624592776139570 & 0.3974578399896733\end{array}$ $\begin{array}{llll}0.8010109111919422 & 0.7626046518688326 & 0.3973472665138009\end{array}$ $\begin{array}{llll}0.0507210498710097 & 0.0129283676690521 & 0.5436174261481078\end{array}$ $\begin{array}{llll}0.5489114319848754 & 0.5108214942889292 & 0.5424348271575983\end{array}$ $\begin{array}{llll}0.2322334032740830 & 0.2015418388778005 & 0.0202776658195892\end{array}$ $\begin{array}{llll}0.7322066834549795 & 0.7015729602088752 & 0.0202786569036770\end{array}$ $\begin{array}{llll}0.9860237222166223 & 0.9500577105354122 & 0.1652318181984519\end{array}$ $\begin{array}{llll}0.4860515518574302 & 0.4499364161558008 & 0.1652389205966982\end{array}$ $\begin{array}{llll}0.2364544703850782 & 0.1996047855284959 & 0.3097908067901385\end{array}$ $\begin{array}{llll}0.7372009929739869 & 0.6993661182846633 & 0.3097125993974907\end{array}$ $\begin{array}{llll}0.9839244393547679 & 0.9514370085121958 & 0.4560633494129661\end{array}$ $\begin{array}{llll}0.4887423223734623 & 0.4474168557527363 & 0.4543827953091927\end{array}$ $\begin{array}{llll}0.3078840266743624 & 0.3447664366894003 & 0.0469109819912896\end{array}$ $\begin{array}{llll}0.8079507852872023 & 0.8446767997325332 & 0.0469094039715526\end{array}$ $\begin{array}{lllll}0.0576837789591338 & 0.0941418746128525 & 0.1909215507983482\end{array}$ $\begin{array}{llll}0.5575986914723393 & 0.5941493182660073 & 0.1909192977425115\end{array}$ $\begin{array}{llll}0.3079202783823036 & 0.3439966385982059 & 0.3354411537043586\end{array}$ $\begin{array}{lllll}0.8083995783905126 & 0.8437531749526449 & 0.3352691063018156\end{array}$ $\begin{array}{llll}0.0576746712402410 & 0.0936381822865862 & 0.4820983271799537\end{array}$ $\begin{array}{llll}0.5604463628242442 & 0.5910199585661740 & 0.4794679105688584\end{array}$ $\begin{array}{llll}0.1544422925038169 & 0.1926576132152009 & 0.0824205624941720\end{array}$ $\begin{array}{llll}0.6544445452200188 & 0.6926454233432373 & 0.0824159599207122\end{array}$ $\begin{array}{llll}0.9056305310139907 & 0.9419866103683627 & 0.2274225565576092\end{array}$ $\begin{array}{lllll}0.4054985304923229 & 0.4420318331442504 & 0.2274045919989781\end{array}$ $\begin{array}{llll}0.1561891875046391 & 0.1915117638536107 & 0.3719621743181136\end{array}$ $\begin{array}{llll}0.6568821017910854 & 0.6911278145314008 & 0.3718216570368778\end{array}$ $\begin{array}{llll}0.9042063702156100 & 0.9397479387671909 & 0.5162117608785751\end{array}$ $\begin{array}{lllll}0.4051113022775764 & 0.4428257057267911 & 0.5165830835208857\end{array}$ $\begin{array}{llll}0.1552948554322935 & 0.6920315251402170 & 0.0468946823967080\end{array}$ $\begin{array}{llll}0.6553577002213219 & 0.1919924633379580 & 0.0469083491595673\end{array}$ $\begin{array}{llll}0.9057886435107539 & 0.4424871301218804 & 0.1908984857958364\end{array}$ $\begin{array}{lllll}0.4058698918163726 & 0.9424366107552247 & 0.1909103389055463\end{array}$ $\begin{array}{llll}0.1558054336854513 & 0.6919969178024632 & 0.3355212085619551\end{array}$ $\begin{array}{llll}0.6560265124178785 & 0.1917000048812879 & 0.3355419327473589\end{array}$ 
$\begin{array}{llll}0.9071875438370584 & 0.4420825140801170 & 0.4813266503703280\end{array}$ $\begin{array}{llll}0.4045814728902894 & 0.9379418794797593 & 0.4802546760561591\end{array}$ $\begin{array}{llll}0.3074797596938392 & 0.8452997682695650 & 0.0824618810187292\end{array}$ $\begin{array}{llll}0.8074110769006003 & 0.3453900829245338 & 0.0824825873389113\end{array}$ $\begin{array}{llll}0.0581359018961474 & 0.5943000501122144 & 0.2274167164101475\end{array}$ $\begin{array}{llll}0.5581007476120282 & 0.0943476568666119 & 0.2274373647663189\end{array}$ $\begin{array}{llll}0.3084460957869239 & 0.8436505478564205 & 0.3719940636829998\end{array}$ $\begin{array}{llll}0.8090784650112940 & 0.3432398297814783 & 0.3718003023178892\end{array}$ $\begin{array}{lllll}0.0618148532886467 & 0.5932451126338719 & 0.5167552794190337\end{array}$ $\begin{array}{llll}0.5627961708675702 & 0.0861557681264757 & 0.5149063337621238\end{array}$ $\begin{array}{lllll}0.2984908733326506 & 0.7675710051023612 & 0.0202839244935106\end{array}$ $\begin{array}{llll}0.7986251916307715 & 0.2675653750409702 & 0.0203156117407187\end{array}$ $\begin{array}{llll}0.0499973139499251 & 0.5140350314109948 & 0.1652404488043858\end{array}$ $\begin{array}{lllll}0.5500096222542861 & 0.0140307999115684 & 0.1652608211799067\end{array}$ $\begin{array}{llll}0.3005072415286337 & 0.7630755910383987 & 0.3098147599544404\end{array}$ $\begin{array}{llll}0.8006154665701418 & 0.2625082331980045 & 0.3097825340503182\end{array}$ $\begin{array}{llll}0.0506931613527355 & 0.5146081955999873 & 0.4556130274843365\end{array}$ $\begin{array}{lllll}0.5517006234746857 & 0.0028038001063388 & 0.4548766114257622\end{array}$ $\begin{array}{llll}0.2376704282556023 & 0.7004451777584197 & 0.1078566622909702\end{array}$ $\begin{array}{lllll}0.7375109976173881 & 0.2005232452594927 & 0.1078620572972013\end{array}$ $\begin{array}{lllll}0.9875263020681651 & 0.4497181247603447 & 0.2529292956325535\end{array}$ $\begin{array}{llll}0.4875658675839664 & 0.9496713979258652 & 0.2529440768495965\end{array}$ $\begin{array}{llll}0.2371296692516320 & 0.6992007718072484 & 0.3974479747170784\end{array}$ $\begin{array}{llll}0.7376069636415722 & 0.1992216691978724 & 0.3975214464165535\end{array}$ $\begin{array}{llll}0.9875708763688025 & 0.4488646869664203 & 0.5430505647160443\end{array}$ $\begin{array}{lllll}0.4834188599138010 & 0.9459832947152331 & 0.5427628315626271\end{array}$ $\begin{array}{lllll}0.4958235861354197 & 0.6327868762177511 & 0.6426072532539305\end{array}$ $\begin{array}{llll}0.5513985839588944 & 0.4863412841397955 & 0.6802248000067298\end{array}$ $\begin{array}{llll}0.5714845296091978 & 0.3110144880451883 & 0.6670912434561472\end{array}$ $\begin{array}{lllll}0.5308103786874517 & 0.2830755384770131 & 0.6164037135368067\end{array}$ $\begin{array}{llll}0.3291940326292841 & 0.1992097313433120 & 0.0905770484445739\end{array}$ $\begin{array}{llll}0.8292242860774008 & 0.6991540410056412 & 0.0905769490831533\end{array}$ $\begin{array}{llll}0.0800270900837303 & 0.9491929112704730 & 0.2356914887987493\end{array}$ $\begin{array}{llll}0.5798842272662218 & 0.4493273731010065 & 0.2357109674023402\end{array}$ $\begin{array}{llll}0.3303982515919587 & 0.1991817988987230 & 0.3802228576531327\end{array}$ $\begin{array}{llll}0.8308623676407956 & 0.6991307575448820 & 0.3801676937620069\end{array}$ $\begin{array}{llll}0.0793325409481412 & 0.9487810006384755 & 0.5266152803581640\end{array}$ $\begin{array}{llll}0.5807288039339438 & 0.4476317403696166 & 0.5250272984880872\end{array}$ $\begin{array}{llll}0.2973481151169660 & 0.1713596443137957 & 0.0370939837010308\end{array}$ $\begin{array}{llll}0.7973337185936613 & 0.6713481275390727 & 0.0370932554209865\end{array}$ $\begin{array}{llll}0.0497031856222653 & 0.9201015661103298 & 0.1823558069857650\end{array}$ $\begin{array}{llll}0.5496840662455044 & 0.4200797071167161 & 0.1823761339352057\end{array}$ $\begin{array}{llll}0.3001331654027349 & 0.1698207478378503 & 0.3269093818683177\end{array}$ $\begin{array}{llll}0.8007891743644685 & 0.6695705598042792 & 0.3268572562100397\end{array}$ $\begin{array}{llll}0.0481401538510689 & 0.9206580303524116 & 0.4730919174021045\end{array}$ $\begin{array}{llll}0.5519527708025678 & 0.4174293017752979 & 0.4716420555219879\end{array}$ $\begin{array}{lllll}0.2301835432212690 & 0.3614655807699397 & 0.0556012533605888\end{array}$ $\begin{array}{llll}0.7302384814645905 & 0.8614246520487686 & 0.0555984591399431\end{array}$ $\begin{array}{llll}0.9812208588024102 & 0.1108319835653851 & 0.2005556961667619\end{array}$ $\begin{array}{llll}0.4811306425691570 & 0.6108527483034358 & 0.2005558269253562\end{array}$ $\begin{array}{llll}0.2314588500110845 & 0.3606538496113408 & 0.3450611862859519\end{array}$ $\begin{array}{llll}0.7320590719943444 & 0.8604181975377282 & 0.3449773119214474\end{array}$ $\begin{array}{llll}0.9805463946134989 & 0.1106433760011386 & 0.4913491116310075\end{array}$ $\begin{array}{llll}0.4842232351834275 & 0.6083530926902203 & 0.4894529066374153\end{array}$ $\begin{array}{llll}0.1376850740564319 & 0.2693082409957606 & 0.0728987639847132\end{array}$ $\begin{array}{lllll}0.6376952394269767 & 0.7692965594065131 & 0.0728977860759394\end{array}$ 
$\begin{array}{llll}0.8888647961386453 & 0.0183717396226932 & 0.2177124743263232\end{array}$ $\begin{array}{llll}0.3887597022878543 & 0.5184155727791809 & 0.2177074933399556\end{array}$ $\begin{array}{llll}0.1392922565845821 & 0.2680466845299123 & 0.3623102971838798\end{array}$ $\begin{array}{lllll}0.6398714463566036 & 0.7677116202621314 & 0.3621842114381447\end{array}$ $\begin{array}{lllll}0.8876128307735157 & 0.0186523152940814 & 0.5085976831162775\end{array}$ $\begin{array}{llll}0.3894329891700344 & 0.5183684284167702 & 0.5066540643277282\end{array}$ $\begin{array}{llll}0.1386030789126370 & 0.7696956703514788 & 0.0556031491277944\end{array}$ $\begin{array}{llll}0.6386213571612409 & 0.2697007896274276 & 0.0556081019764860\end{array}$ $\begin{array}{llll}0.8891277173621299 & 0.5188795729353693 & 0.2005710074834121\end{array}$ $\begin{array}{llll}0.3892096047334008 & 0.0188230887709786 & 0.2005889761240596\end{array}$ $\begin{array}{llll}0.1392776852983529 & 0.7684670377500230 & 0.3450967642733963\end{array}$ $\begin{array}{llll}0.6395083413300091 & 0.2680794946452027 & 0.3451763333906245\end{array}$ $\begin{array}{llll}0.8904139758109775 & 0.5189792682100551 & 0.4906794934263655\end{array}$ $\begin{array}{llll}0.3892606170494577 & 0.0135852145541210 & 0.4900285573739856\end{array}$ $\begin{array}{llll}0.2308254387131450 & 0.8621433803330180 & 0.0729153475783485\end{array}$ $\begin{array}{llll}0.7307651069528579 & 0.3622162999107205 & 0.0729345063235207\end{array}$ $\begin{array}{llll}0.9816985046677724 & 0.6111483351857198 & 0.2177227648656485\end{array}$ $\begin{array}{llll}0.4817056445053348 & 0.1111409187410460 & 0.2177266608974439\end{array}$ $\begin{array}{llll}0.2319924912080606 & 0.8605083296443716 & 0.3623072677734080\end{array}$ $\begin{array}{lllll}0.7324168285883018 & 0.3601516115896926 & 0.3622409684399036\end{array}$ $\begin{array}{llll}0.9836171713266693 & 0.6105436800812337 & 0.5082246957569577\end{array}$ $\begin{array}{llll}0.4839293556726768 & 0.1036586133312993 & 0.5068898601758796\end{array}$ $\begin{array}{llll}0.3287228289036067 & 0.7024969013481354 & 0.0371113528770670\end{array}$ $\begin{array}{llll}0.8288169150129151 & 0.2025165109572269 & 0.0371498120841056\end{array}$ $\begin{array}{lllll}0.0798957788930463 & 0.4503457080118901 & 0.1823637451604133\end{array}$ $\begin{array}{llll}0.5799371366280310 & 0.9503260836804546 & 0.1823794584517808\end{array}$ $\begin{array}{llll}0.3302086566586291 & 0.6994899479590841 & 0.3269540733544261\end{array}$ $\begin{array}{llll}0.8303880356589822 & 0.1990176729672536 & 0.3269462881914088\end{array}$ $\begin{array}{llll}0.0808951423552832 & 0.4503131971742182 & 0.4725956457185614\end{array}$ $\begin{array}{lllll}0.5818752203412429 & 0.9406178221974730 & 0.4724331009552268\end{array}$ $\begin{array}{llll}0.3008256121343066 & 0.6706404756754873 & 0.0905818668020413\end{array}$ $\begin{array}{llll}0.8007464207431352 & 0.1707338161591372 & 0.0906132965716260\end{array}$ $\begin{array}{llll}0.0507589003026820 & 0.4200186397019788 & 0.2356933422314950\end{array}$ $\begin{array}{lllll}0.5508194172507268 & 0.9200182805553917 & 0.2357151912237364\end{array}$ $\begin{array}{llll}0.3006454594138885 & 0.6694820384182785 & 0.3802796442324837\end{array}$ $\begin{array}{llll}0.8010544750435010 & 0.1693622917523691 & 0.3803309645907003\end{array}$ $\begin{array}{llll}0.0517747575100272 & 0.4194530845660310 & 0.5260176186018508\end{array}$ $\begin{array}{llll}0.5489021046970822 & 0.9148919749973020 & 0.5260090513110682\end{array}$ $\begin{array}{llll}0.2488404298697742 & 0.2501849256365430 & 0.0640217196160979\end{array}$ $\begin{array}{llll}0.7488598990738967 & 0.7501625624029662 & 0.0640217185459800\end{array}$ $\begin{array}{lllll}-0.0000005564440371 & 0.9995761482579542 & 0.2090763720246247\end{array}$ $\begin{array}{llll}0.4998931804028381 & 0.4996274304914367 & 0.2090842379355530\end{array}$ $\begin{array}{llll}0.2503435986533485 & 0.2493921607314421 & 0.3536127919556385\end{array}$ $\begin{array}{llll}0.7509022690192015 & 0.7491825240709082 & 0.3535456185251120\end{array}$ $\begin{array}{llll}0.9989955259287088 & 0.9995638559992738 & 0.4999778165390221\end{array}$ $\begin{array}{llll}0.5017646452673186 & 0.4978138141946241 & 0.4981868069986151\end{array}$ $\begin{array}{llll}0.2498817123845420 & 0.7510112368611218 & 0.0640354498518473\end{array}$ $\begin{array}{llll}0.7498812412004395 & 0.2510628538142475 & 0.0640564592574692\end{array}$ $\begin{array}{llll}0.0003856505614216 & 0.5000701954147433 & 0.2090812097875990\end{array}$ $\begin{array}{llll}0.5004482945193151 & 0.0000327536872794 & 0.2091004400702631\end{array}$ $\begin{array}{llll}0.2506099341117671 & 0.7494356020960325 & 0.3536456105090823\end{array}$ $\begin{array}{llll}0.7508768012222972 & 0.2491153719320305 & 0.3536580929268049\end{array}$ $\begin{array}{llll}0.0017897006188178 & 0.4997946526450351 & 0.4994141495173234\end{array}$ $\begin{array}{llll}0.5011632385999020 & 0.9928441203637610 & 0.4988899361813059\end{array}$ $\begin{array}{llll}0.4825635033949656 & 0.4416677201562816 & 0.5999143801253867\end{array}$ $\begin{array}{llll}0.4846365846581160 & 0.5456439103330343 & 0.6071468973681315\end{array}$ 
$\begin{array}{llll}0.5051851047164754 & 0.5557531404990450 & 0.6372236552856652\end{array}$ $\begin{array}{llll}0.5347866395536277 & 0.4744129683699718 & 0.6579205541678222\end{array}$ $\begin{array}{llll}0.5454273823879118 & 0.3752450803766152 & 0.6505262667962322\end{array}$ $\begin{array}{llll}0.5215636075741423 & 0.3593747392262004 & 0.6226873920184381\end{array}$ $\begin{array}{llll}0.5171719012989464 & 0.1372154682853793 & 0.1044770654086061\end{array}$ $\begin{array}{llll}0.0171860231632169 & 0.6372496087211823 & 0.1044745121782137\end{array}$ $\begin{array}{lllll}0.2684432961057099 & 0.8865681420788517 & 0.2493276707378544\end{array}$ $\begin{array}{llll}0.7683836873941935 & 0.3865081631005227 & 0.2493315853215114\end{array}$ $\begin{array}{llll}0.5196084804630593 & 0.1338656659848524 & 0.3938537562037394\end{array}$ $\begin{array}{llll}0.0190693927930557 & 0.6364362139596346 & 0.3939677268576975\end{array}$ $\begin{array}{llll}0.2696517994230082 & 0.8818426932004698 & 0.5403468032016224\end{array}$ $\begin{array}{llll}0.7689262424202107 & 0.3815869002470689 & 0.5397929317372012\end{array}$ $\begin{array}{llll}0.3639995069792865 & 0.9818597212323389 & 0.0229623733798391\end{array}$ $\begin{array}{llll}0.8640050634798512 & 0.4818482407013250 & 0.0229472437424539\end{array}$ $\begin{array}{llll}0.1133449694742951 & 0.7316396465420305 & 0.1685131127874572\end{array}$ $\begin{array}{llll}0.6133589218504504 & 0.2315396321017080 & 0.1685909624348054\end{array}$ $\begin{array}{llll}0.3638389077724103 & 0.9812157996516815 & 0.3131996880376439\end{array}$ $\begin{array}{llll}0.8639460219320440 & 0.4811167982390971 & 0.3131671858361755\end{array}$ $\begin{array}{llll}0.1124359624492975 & 0.7327732940420660 & 0.4584667458336671\end{array}$ $\begin{array}{lllll}0.6134353208090548 & 0.2294956794377617 & 0.4579605072073638\end{array}$ $\begin{array}{llll}0.1312162991141025 & 0.5098567783897162 & 0.0185838553496011\end{array}$ $\begin{array}{llll}0.6312638497235605 & 0.0098170545306405 & 0.0185860852555205\end{array}$ $\begin{array}{llll}0.8794127128193084 & 0.2601548667107437 & 0.1643487496650682\end{array}$ $\begin{array}{llll}0.3793829281429798 & 0.7601308402053352 & 0.1643477040565333\end{array}$ $\begin{array}{llll}0.1296952999865472 & 0.5098306226461080 & 0.3088328982827301\end{array}$ $\begin{array}{llll}0.6297284482090511 & 0.0099451660094370 & 0.3088488530146416\end{array}$ $\begin{array}{llll}0.8817211892846460 & 0.2575543093940683 & 0.4543030038677892\end{array}$ $\begin{array}{llll}0.3803921918350784 & 0.7585497722772350 & 0.4535313643301341\end{array}$ $\begin{array}{llll}0.9900410462800340 & 0.3703665027088565 & 0.1095588693373072\end{array}$ $\begin{array}{llll}0.4900420294303067 & 0.8703827423025488 & 0.1095537662530816\end{array}$ $\begin{array}{llll}0.7396455672890196 & 0.1205838906055478 & 0.2539146771475043\end{array}$ $\begin{array}{llll}0.2396342073157343 & 0.6205250324524614 & 0.2539299779297068\end{array}$ $\begin{array}{llll}0.9910764324273668 & 0.3696545945279585 & 0.3988283434186677\end{array}$ $\begin{array}{llll}0.4905671970350030 & 0.8693956900025362 & 0.3983844704744674\end{array}$ $\begin{array}{llll}0.7442850514461100 & 0.1100000057493379 & 0.5470388746051966\end{array}$ $\begin{array}{llll}0.2455591382215560 & 0.6170749601963100 & 0.5445489686531174\end{array}$ $\begin{array}{llll}0.9902078500955541 & 0.8687270898366426 & 0.0185916689159951\end{array}$ $\begin{array}{llll}0.4902657453089465 & 0.3686871854528940 & 0.0185914549352120\end{array}$ $\begin{array}{llll}0.7398433302624015 & 0.6206742204718455 & 0.1643574868085572\end{array}$ $\begin{array}{llll}0.2399106429040722 & 0.1206995295979307 & 0.1643815379441740\end{array}$ $\begin{array}{llll}0.9902965320734021 & 0.8701884639712446 & 0.3088184325555122\end{array}$ $\begin{array}{llll}0.4903567032753541 & 0.3700608256280991 & 0.3089062949924926\end{array}$ $\begin{array}{llll}0.7441298752994728 & 0.6176988452805696 & 0.4535543024642424\end{array}$ $\begin{array}{llll}0.2405845897466014 & 0.1187817022152709 & 0.4537187588339294\end{array}$ $\begin{array}{llll}0.1297385628396756 & 0.0099133780937988 & 0.1095284359601503\end{array}$ $\begin{array}{llll}0.6296479674585737 & 0.5099967896947589 & 0.1095398817643877\end{array}$ $\begin{array}{llll}0.8796335987733466 & 0.7603716205223958 & 0.2539073707202697\end{array}$ $\begin{array}{llll}0.3795355782081828 & 0.2603771580909799 & 0.2539152075579242\end{array}$ $\begin{array}{lllll}0.1300362961241790 & 0.0091205200859976 & 0.3987304238768480\end{array}$ $\begin{array}{llll}0.6308046119310855 & 0.5090517975875314 & 0.3985422652039936\end{array}$ $\begin{array}{llll}0.8863079043205025 & 0.7569596954740410 & 0.5457541437514838\end{array}$ $\begin{array}{llll}0.3891217191891982 & 0.2541436250739646 & 0.5431649966079457\end{array}$ $\begin{array}{llll}0.5181963527884335 & 0.6359525466825281 & 0.0229858937836135\end{array}$ $\begin{array}{lllll}0.0183200531249020 & 0.1359256789796619 & 0.0230648676009064\end{array}$ $\begin{array}{llll}0.2684461312170238 & 0.3865629202985147 & 0.1685610196624548\end{array}$ $\begin{array}{llll}0.7684186792692036 & 0.8866110971055062 & 0.1685416823255167\end{array}$ 
$\begin{array}{llll}0.5188119939472782 & 0.6358706638981010 & 0.3132900141239846\end{array}$ $\begin{array}{llll}0.0187910483426231 & 0.1359629057418240 & 0.3131934697826985\end{array}$ $\begin{array}{llll}0.2692584194151013 & 0.3847299523636628 & 0.4580961579717519\end{array}$ $\begin{array}{llll}0.7680731457449652 & 0.8841407745306414 & 0.4583733855744379\end{array}$ $\begin{array}{llll}0.3627533062966652 & 0.4826668695296077 & 0.1044480820976385\end{array}$ $\begin{array}{llll}0.8627221834525028 & 0.9827407968067055 & 0.1044785201366713\end{array}$ $\begin{array}{llll}0.1133797480689752 & 0.2315848474659290 & 0.2493189515103260\end{array}$ $\begin{array}{llll}0.6134060871053924 & 0.7315559800133178 & 0.2493211580633880\end{array}$ $\begin{array}{llll}0.3635246752711953 & 0.4810376659232491 & 0.3939732655880563\end{array}$ $\begin{array}{lllll}0.8637852017714459 & 0.9810679417839187 & 0.3941454393827150\end{array}$ $\begin{array}{llll}0.1169280527673805 & 0.2300517541169572 & 0.5396840799336416\end{array}$ $\begin{array}{llll}0.6168850297045281 & 0.7253673245526480 & 0.5408216155608182\end{array}$ $\begin{array}{llll}0.4401820394446486 & 0.1626587467250100 & 0.0804025992205129\end{array}$ $\begin{array}{llll}0.9401919166215976 & 0.6626567010360085 & 0.0804001774312388\end{array}$ $\begin{array}{lllll}0.1909615031949390 & 0.9126459512771254 & 0.2253975563471579\end{array}$ $\begin{array}{lllll}0.6907841104512809 & 0.4129029231315327 & 0.2254101772580721\end{array}$ $\begin{array}{llll}0.4411744068747780 & 0.1629863706619848 & 0.3699121464013539\end{array}$ $\begin{array}{lllll}0.9418490278647436 & 0.6625687716184001 & 0.3699362654240647\end{array}$ $\begin{array}{llll}0.1907366523259295 & 0.9095687552067424 & 0.5162925227094936\end{array}$ $\begin{array}{llll}0.6916263941454011 & 0.4136060073417065 & 0.5153776341701832\end{array}$ $\begin{array}{llll}0.3355101362108778 & 0.0598426354153819 & 0.0471353921680986\end{array}$ $\begin{array}{llll}0.8354577391775574 & 0.5598478152088470 & 0.0471309081210688\end{array}$ $\begin{array}{llll}0.0865301663032196 & 0.8088931639719090 & 0.1925189966682899\end{array}$ $\begin{array}{llll}0.5865484234397552 & 0.3088863495180449 & 0.1925632010413806\end{array}$ $\begin{array}{llll}0.3370861846478059 & 0.0586293713804870 & 0.3371351019472630\end{array}$ $\begin{array}{llll}0.8376244393331219 & 0.5584384514435723 & 0.3370922315594924\end{array}$ $\begin{array}{lllll}0.0852839888040361 & 0.8090238096136886 & 0.4828509959973631\end{array}$ $\begin{array}{llll}0.5878000272627708 & 0.3066701932706146 & 0.4818894030971675\end{array}$ $\begin{array}{llll}0.1423413888744168 & 0.4146495398305738 & 0.0331532861234835\end{array}$ $\begin{array}{llll}0.6424031546152350 & 0.9146075722542382 & 0.0331519572250790\end{array}$ $\begin{array}{llll}0.8898511899293428 & 0.1666910790079879 & 0.1790167927693293\end{array}$ $\begin{array}{lllll}0.3897550562532500 & 0.6667295688301885 & 0.1790173586233580\end{array}$ $\begin{array}{lllll}0.1401860696514861 & 0.4163357289064287 & 0.3235004514159521\end{array}$ $\begin{array}{llll}0.6404210941292011 & 0.9163663488786892 & 0.3235212156846847\end{array}$ $\begin{array}{llll}0.8907901786487957 & 0.1652518870440506 & 0.4693805492794538\end{array}$ $\begin{array}{llll}0.3917310960436518 & 0.6649097932535390 & 0.4681778269295750\end{array}$ $\begin{array}{lllll}0.0832942441778974 & 0.3598447993098328 & 0.0946264834358245\end{array}$ $\begin{array}{llll}0.5833188489617077 & 0.8598275873705774 & 0.0946253593192019\end{array}$ $\begin{array}{lllll}0.8332222228441750 & 0.1099033534533615 & 0.2391740624896576\end{array}$ $\begin{array}{llll}0.3331318560673246 & 0.6099227045085249 & 0.2391774934694236\end{array}$ $\begin{array}{llll}0.0841285566912474 & 0.3592252687888903 & 0.3838743990492202\end{array}$ $\begin{array}{llll}0.5842112724918556 & 0.8589423024109446 & 0.3837272584396145\end{array}$ $\begin{array}{llll}0.8376639311464245 & 0.1017885046306512 & 0.5322438734838654\end{array}$ $\begin{array}{llll}0.3335206509505293 & 0.6108738907897970 & 0.5277205749498570\end{array}$ $\begin{array}{llll}0.0854310641530438 & 0.8575868623356302 & 0.0331673549358676\end{array}$ $\begin{array}{llll}0.5854731546166625 & 0.3575616027143018 & 0.0331626622462247\end{array}$ $\begin{array}{llll}0.8331462369452691 & 0.6103985361508604 & 0.1790767355061299\end{array}$ $\begin{array}{llll}0.3331937237898871 & 0.1103871534244606 & 0.1791136516242018\end{array}$ $\begin{array}{llll}0.0837892241716632 & 0.8596081278758246 & 0.3234959559388192\end{array}$ $\begin{array}{llll}0.5837463919445688 & 0.3594999576207568 & 0.3236542450335355\end{array}$ $\begin{array}{llll}0.8359894340331343 & 0.6090196080453534 & 0.4687895051003689\end{array}$ $\begin{array}{lllll}0.3352520878299124 & 0.1053109497993871 & 0.4685760740213833\end{array}$ $\begin{array}{llll}0.1402069031700710 & 0.9166599753392244 & 0.0946144835469720\end{array}$ $\begin{array}{llll}0.6401407229008528 & 0.4167184012215699 & 0.0946300872694186\end{array}$ $\begin{array}{llll}0.8902089192610592 & 0.6668291563512311 & 0.2391935133604961\end{array}$ $\begin{array}{llll}0.3901526014177326 & 0.1668324593039720 & 0.2391810616709597\end{array}$ 
$\begin{array}{llll}0.1406112370780514 & 0.9159111955315755 & 0.3838153462783367\end{array}$ $\begin{array}{llll}0.6414799943512489 & 0.4154446681937858 & 0.3838643392728081\end{array}$ $\begin{array}{llll}0.8968031479189232 & 0.6625506440836447 & 0.5309449225191808\end{array}$ $\begin{array}{llll}0.3984208502817893 & 0.1574433132706939 & 0.5298272327721031\end{array}$ $\begin{array}{llll}0.4401686782666965 & 0.6645096257784436 & 0.0471568108452165\end{array}$ $\begin{array}{llll}0.9402093061402366 & 0.1645576478423979 & 0.0472209455792534\end{array}$ $\begin{array}{llll}0.1911031850468269 & 0.4134375924164269 & 0.1925461001870133\end{array}$ $\begin{array}{llll}0.6911574817730944 & 0.9134419803682154 & 0.1925442143725371\end{array}$ $\begin{array}{llll}0.4413686261315448 & 0.6625315084228015 & 0.3372020145390366\end{array}$ $\begin{array}{llll}0.9416100128039762 & 0.1620868999171326 & 0.3371401147708842\end{array}$ $\begin{array}{llll}0.1927800997919335 & 0.4120096391107246 & 0.4824512099068128\end{array}$ $\begin{array}{llll}0.6924540772343594 & 0.9058154692784328 & 0.4824598088081883\end{array}$ $\begin{array}{llll}0.3372466076016539 & 0.5597228043379474 & 0.0803921227641938\end{array}$ $\begin{array}{lllll}0.8373032922139957 & 0.0597638901094576 & 0.0804211261495757\end{array}$ $\begin{array}{llll}0.0871382796985190 & 0.3091329740349406 & 0.2253976155680512\end{array}$ $\begin{array}{lllll}0.5873492094822319 & 0.8090917483929527 & 0.2254138691428864\end{array}$ $\begin{array}{llll}0.3374230343778987 & 0.5584566411801934 & 0.3699985521039844\end{array}$ $\begin{array}{lllll}0.8377428087089954 & 0.0583081216119176 & 0.3701273697108309\end{array}$ $\begin{array}{llll}0.0890890776791607 & 0.3085236671735531 & 0.5156823564979981\end{array}$ $\begin{array}{llll}0.5869182674690587 & 0.8031134132372476 & 0.5163142619841521\end{array}$ $\begin{array}{llll}0.6108067283059926 & 0.1028667768135825 & 0.0956967072441589\end{array}$ $\begin{array}{lllll}0.1108026440614977 & 0.6029289331232927 & 0.0956923716370521\end{array}$ $\begin{array}{llll}0.3619957722073652 & 0.8520490874095727 & 0.2404130961967131\end{array}$ $\begin{array}{llll}0.8619156038887905 & 0.3520602521094188 & 0.2404147380601979\end{array}$ $\begin{array}{lllll}0.6128464385813646 & 0.1003352177216241 & 0.3849143850055010\end{array}$ $\begin{array}{lllll}0.1127709789621855 & 0.6018158059852484 & 0.3851525566295732\end{array}$ $\begin{array}{llll}0.3627939186850470 & 0.8475467459970132 & 0.5310572343399053\end{array}$ $\begin{array}{llll}0.8615382299726088 & 0.3530120615038531 & 0.5314987589144503\end{array}$ $\begin{array}{lllll}0.3970583443036811 & 0.8885886676717215 & 0.0319153350780684\end{array}$ $\begin{array}{lllll}0.8970702921375513 & 0.3885744449048454 & 0.0318956171490070\end{array}$ $\begin{array}{llll}0.1481487881000136 & 0.6378233533058228 & 0.1772829875485334\end{array}$ $\begin{array}{llll}0.6481057241922509 & 0.1377760987202669 & 0.1773951374643363\end{array}$ $\begin{array}{llll}0.3987624968294031 & 0.8874359374727996 & 0.3220221945606420\end{array}$ $\begin{array}{llll}0.8987143840828914 & 0.3873970275069789 & 0.3220053686391859\end{array}$ $\begin{array}{llll}0.1471439730312884 & 0.6385713445062192 & 0.4668211140789414\end{array}$ $\begin{array}{lllll}0.6479597461260989 & 0.1358666987494911 & 0.4668110490223202\end{array}$ $\begin{array}{lllll}0.0530895659810034 & 0.5577098739897839 & 0.0013307639797921\end{array}$ $\begin{array}{llll}0.5531111638827342 & 0.0576847320089912 & 0.0013386751724557\end{array}$ $\begin{array}{llll}0.7999013237384415 & 0.3089651513295185 & 0.1473332345588250\end{array}$ $\begin{array}{lllll}0.2998792259307219 & 0.8089607706374744 & 0.1473261182651999\end{array}$ $\begin{array}{llll}0.0502390280765438 & 0.5585505970148900 & 0.2917986130158560\end{array}$ $\begin{array}{lllll}0.5500918942248628 & 0.0587227018057707 & 0.2918705024207999\end{array}$ $\begin{array}{llll}0.8036996174210070 & 0.3048081453453231 & 0.4368476284904133\end{array}$ $\begin{array}{llll}0.2996661098668669 & 0.8081797749908178 & 0.4368792971058240\end{array}$ $\begin{array}{llll}0.9422494109811840 & 0.4494108925684492 & 0.1266934025291413\end{array}$ $\begin{array}{lllll}0.4422539344634664 & 0.9494413052255370 & 0.1266858265225012\end{array}$ $\begin{array}{llll}0.6907894863008198 & 0.2003722434454530 & 0.2708233603708710\end{array}$ $\begin{array}{lllll}0.1908060915880364 & 0.7002457159847151 & 0.2708617843270368\end{array}$ $\begin{array}{llll}0.9433999252386930 & 0.4486089205192036 & 0.4160055148427009\end{array}$ $\begin{array}{llll}0.4418994998663247 & 0.9487076338947791 & 0.4154363330940748\end{array}$ $\begin{array}{llll}0.7005484777278627 & 0.1826636567053425 & 0.5658449109631654\end{array}$ $\begin{array}{llll}0.2021163981498881 & 0.6927910242943185 & 0.5625603391837644\end{array}$ $\begin{array}{llll}0.9423599291201737 & 0.9468629669226228 & 0.0013392104074552\end{array}$ $\begin{array}{llll}0.4424058301713926 & 0.4468326685574048 & 0.0013433752737976\end{array}$ $\begin{array}{llll}0.6909885269186953 & 0.7002284623540651 & 0.1473538386659709\end{array}$ $\begin{array}{llll}0.1910998704477448 & 0.2002758377892850 & 0.1473847378595691\end{array}$ 
$\begin{array}{llll}0.9416407999671850 & 0.9496506890021461 & 0.2917860750197401\end{array}$ $\begin{array}{llll}0.4417308332395810 & 0.4496188963827401 & 0.2918984701508108\end{array}$ $\begin{array}{llll}0.6968445185744880 & 0.6957649353148418 & 0.4361013127661248\end{array}$ $\begin{array}{llll}0.1933206743426040 & 0.1989467617038366 & 0.4369042435345358\end{array}$ $\begin{array}{llll}0.0506832445264389 & 0.0577765850126296 & 0.1266597492791645\end{array}$ $\begin{array}{llll}0.5505928063917190 & 0.5578524091920004 & 0.1266704595989180\end{array}$ $\begin{array}{llll}0.7999464050753160 & 0.8092429605012618 & 0.2708486796576446\end{array}$ $\begin{array}{lllll}0.2998276110928154 & 0.3092169971780191 & 0.2708493580506028\end{array}$ $\begin{array}{llll}0.0509831176418621 & 0.0569440223529934 & 0.4158705521646028\end{array}$ $\begin{array}{llll}0.5516591196255587 & 0.5573446142634115 & 0.4156554789110912\end{array}$ $\begin{array}{llll}0.8085123198122193 & 0.8043299312787967 & 0.5631214505214299\end{array}$ $\begin{array}{llll}0.3136007692339751 & 0.3029798293296438 & 0.5611415272728216\end{array}$ $\begin{array}{llll}0.6114094150448633 & 0.6029920956762277 & 0.0319608165029138\end{array}$ $\begin{array}{llll}0.1115149403963310 & 0.1029864322211129 & 0.0320509997262014\end{array}$ $\begin{array}{llll}0.3622347078234441 & 0.3517656187246949 & 0.1773554350857827\end{array}$ $\begin{array}{llll}0.8622420327235416 & 0.8517745407205083 & 0.1773125258962443\end{array}$ $\begin{array}{llll}0.6125519117677832 & 0.6009573688094849 & 0.3221425041906747\end{array}$ $\begin{array}{lllll}0.1125371330122773 & 0.1011798157858411 & 0.3220114532640216\end{array}$ $\begin{array}{lllll}0.3638125542942828 & 0.3484638479270434 & 0.4664948433865338\end{array}$ $\begin{array}{llll}0.8610237922923610 & 0.8520474370147831 & 0.4671459235580523\end{array}$ $\begin{array}{llll}0.3970204982624119 & 0.3890787499538540 & 0.0956515996926413\end{array}$ $\begin{array}{llll}0.8970082088030374 & 0.8891492432785918 & 0.0956850382072964\end{array}$ $\begin{array}{llll}0.1479162812779549 & 0.1380237353261986 & 0.2404042393782614\end{array}$ $\begin{array}{lllll}0.6478454893607083 & 0.6380516881229421 & 0.2403828342708111\end{array}$ $\begin{array}{llll}0.3981460019253181 & 0.3874005951833293 & 0.3850990753552028\end{array}$ $\begin{array}{llll}0.8983890071494121 & 0.8873846444045820 & 0.3853113833877808\end{array}$ $\begin{array}{llll}0.1490117004646601 & 0.1373142277782843 & 0.5305612897617122\end{array}$ $\begin{array}{llll}0.6507842539360065 & 0.6316472081693378 & 0.5321417293511016\end{array}$ $\begin{array}{llll}0.4806074474046999 & 0.1525460132821943 & 0.1292873025872687\end{array}$ $\begin{array}{lllll}0.9806209258871023 & 0.6525870572581027 & 0.1292852556956941\end{array}$ $\begin{array}{llll}0.2324443549498218 & 0.9016577438834660 & 0.2741997305291050\end{array}$ $\begin{array}{llll}0.7324846158520503 & 0.4013028712427908 & 0.2742067682512133\end{array}$ $\begin{array}{llll}0.4845754447210036 & 0.1458257474357046 & 0.4187278452641126\end{array}$ $\begin{array}{llll}0.9827067517442857 & 0.6515980184567118 & 0.4187945467901532\end{array}$ $\begin{array}{llll}0.2347166858367800 & 0.8960694245395538 & 0.5651959366843102\end{array}$ $\begin{array}{llll}0.7344472322552389 & 0.3862609885001154 & 0.5643211820875742\end{array}$ $\begin{array}{llll}0.3521799845680933 & 0.0169072909708530 & 0.9981831142796432\end{array}$ $\begin{array}{llll}0.8522302101864266 & 0.5169107842280082 & 0.9981722640528887\end{array}$ $\begin{array}{llll}0.0984766005836247 & 0.7679525119997724 & 0.1436666562455335\end{array}$ $\begin{array}{llll}0.5985900963171269 & 0.2677068122289309 & 0.1437324127441536\end{array}$ $\begin{array}{llll}0.3488432870094546 & 0.0173592451657382 & 0.2883294488638996\end{array}$ $\begin{array}{llll}0.8488172899890849 & 0.5172646396278138 & 0.2882964658122299\end{array}$ $\begin{array}{llll}0.0979083980660406 & 0.7703801062762150 & 0.4337801649591433\end{array}$ $\begin{array}{llll}0.5981435041023579 & 0.2656337732735091 & 0.4330756840281956\end{array}$ $\begin{array}{llll}0.1991547144946832 & 0.5279554524686805 & 0.0253031927848184\end{array}$ $\begin{array}{llll}0.6992167098384284 & 0.0279051519184561 & 0.0253014865102076\end{array}$ $\begin{array}{llll}0.9480637548124773 & 0.2778085949713865 & 0.1704323502706952\end{array}$ $\begin{array}{llll}0.4480651709646598 & 0.7777383378098146 & 0.1704321489527397\end{array}$ $\begin{array}{llll}0.1982390137579388 & 0.5276027149809889 & 0.3149357260082604\end{array}$ $\begin{array}{llll}0.6983158530127834 & 0.0277181590820473 & 0.3148940792405762\end{array}$ $\begin{array}{llll}0.9498270700513910 & 0.2756874712103082 & 0.4606073562831496\end{array}$ $\begin{array}{llll}0.4496156952066682 & 0.7758902362496025 & 0.4592292649625499\end{array}$ $\begin{array}{llll}0.9717010380144738 & 0.3022443494226220 & 0.1034565772240343\end{array}$ $\begin{array}{llll}0.4716847114399467 & 0.8022718289651850 & 0.1034560273113141\end{array}$ $\begin{array}{llll}0.7220624128882837 & 0.0518177229558049 & 0.2479250596701923\end{array}$ $\begin{array}{llll}0.2220395628123094 & 0.5517772205013313 & 0.2479268772145311\end{array}$ 
$\begin{array}{llll}0.9726605025450273 & 0.3015865687813063 & 0.3927239192271570\end{array}$ $\begin{array}{llllll}0.4727922645033436 & 0.8009271123749188 & 0.3921816316677321\end{array}$ $\begin{array}{lllll}0.7233228123832367 & 0.0454350113519525 & 0.5389833457916424\end{array}$ $\begin{array}{lllll}0.2266412413613225 & 0.5490975698416692 & 0.5386317945564809\end{array}$ $\begin{array}{lllll}0.9721190754964867 & 0.8007780257033248 & 0.0253056706505609\end{array}$ $\begin{array}{lllll}0.4721772379594505 & 0.3007365116096651 & 0.0253058901476008\end{array}$ $\begin{array}{lllll}0.7223592965567454 & 0.5518663278261650 & 0.1703957765504853\end{array}$ $\begin{array}{lllll}0.2223551922106839 & 0.0519301398467987 & 0.1703976867368446\end{array}$ $\begin{array}{lllll}0.9724480581381392 & 0.8016886932215109 & 0.3149078936403429\end{array}$ $\begin{array}{lllll}0.4725460877130950 & 0.3014830018583725 & 0.3149252358299243\end{array}$ $\begin{array}{lllll}0.7262714178614585 & 0.5493274542428984 & 0.4597491913508004\end{array}$ $\begin{array}{lllll}0.2205782177128312 & 0.0516907091977524 & 0.4595021416990264\end{array}$ $\begin{array}{llllll}0.1979163685259716 & 0.0281706096693341 & 0.1034213635957501\end{array}$ $\begin{array}{lllll}0.6978013593122326 & 0.5282835768383147 & 0.1034287959779703\end{array}$ $\begin{array}{lllll}0.9483653808675830 & 0.7779521808618122 & 0.2478704631865498\end{array}$ $\begin{array}{lllll}0.4482630743274280 & 0.2779787923525149 & 0.2478947724349088\end{array}$ $\begin{array}{lllll}0.1981561691961055 & 0.0274714928219553 & 0.3926140763021979\end{array}$ $\begin{array}{lllll}0.6989463114739231 & 0.5272386681023778 & 0.3923098575315260\end{array}$ $\begin{array}{lllll}0.9542898813725296 & 0.7751256592194790 & 0.5391128408101702\end{array}$ $\begin{array}{lllll}0.4559717472021163 & 0.2713231571116116 & 0.5347520433613530\end{array}$ $\begin{array}{llllll}0.4831848888327045 & 0.6476739316058197 & 0.9982048078475722\end{array}$ $\begin{array}{lllll}0.9833851154466097 & 0.1475668326499014 & 0.9982793192485490\end{array}$ $\begin{array}{lllll}0.2322398999079778 & 0.4013632498926222 & 0.1437069737146594\end{array}$ $\begin{array}{lllll}0.7321222144751469 & 0.9014822129240272 & 0.1436948665088113\end{array}$ $\begin{array}{lllll}0.4827505066806946 & 0.6508925277545925 & 0.2884116258457599\end{array}$ $\begin{array}{lllll}0.9825352553559827 & 0.1512549906435587 & 0.2883291247411939\end{array}$ $\begin{array}{llllll}0.2313279301732999 & 0.4006676372207041 & 0.4334069695192678\end{array}$ $\begin{array}{lllll}0.7313732912810307 & 0.8998334678297562 & 0.4335677556229595\end{array}$ $\begin{array}{llll}0.3475639364714536 & 0.5191211132860293 & 0.1292659795089819\end{array}$ $\begin{array}{lllll}0.8474193008354398 & 0.0192546388317436 & 0.1292952051556419\end{array}$ $\begin{array}{lllll}0.0983786044856947 & 0.2675452870718918 & 0.2741917952320073\end{array}$ $\begin{array}{lllll}0.5983985968322890 & 0.7674269813407039 & 0.2742052870401575\end{array}$ $\begin{array}{llllll}0.3483678363702518 & 0.5171817945800814 & 0.4188269139111371\end{array}$ $\begin{array}{lllll}0.8485801868470565 & 0.0173867953649740 & 0.4189741660296005\end{array}$ $\begin{array}{lllll}0.1055690858478963 & 0.2643863467709713 & 0.5645415880971963\end{array}$ $\begin{array}{lllll}0.6054230391071251 & 0.7614232092482839 & 0.5654654067065430\end{array}$ $\begin{array}{lllll}0.4504436899414060 & 0.4355424737576612 & 0.5753891384127610\end{array}$ $\begin{array}{llll}0.4662672048637764 & 0.6122767789503996 & 0.5873135630535156\end{array}$ 


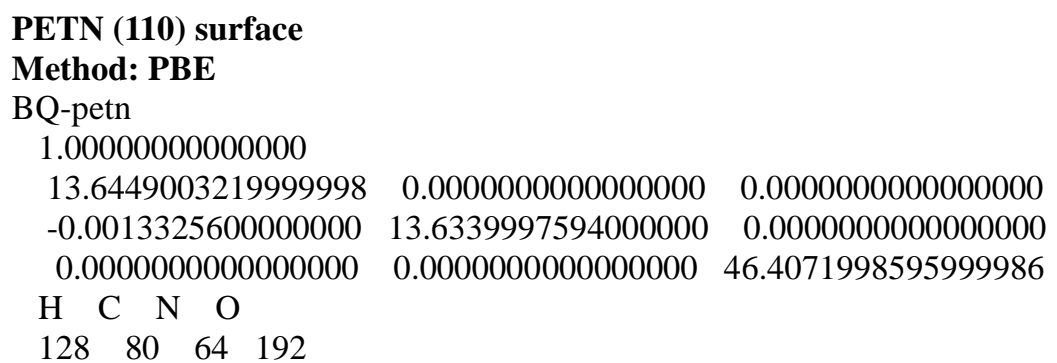

Direct

$\begin{array}{llll}0.3524099776646916 & 0.5331667339957953 & 0.0989226509511773\end{array}$

$\begin{array}{lllll}0.8524099364767803 & 0.5331667342534000 & 0.0989226561233619\end{array}$

$\begin{array}{lllll}0.3522714114224421 & 0.0334915704289491 & 0.2459714905126197\end{array}$

$\begin{array}{lllll}0.8522714387270569 & 0.0334915898128368 & 0.2459715115684634\end{array}$

$\begin{array}{lllll}0.3524204824662978 & 0.5328906478232202 & 0.3927923655713679\end{array}$

$\begin{array}{lllll}0.8524228491553626 & 0.5328935447465828 & 0.3927929871655964\end{array}$

$\begin{array}{lllll}0.3517985748084705 & 0.0335551751951033 & 0.5403024303441026\end{array}$

$\begin{array}{lllll}0.8517999147785343 & 0.0335545931868668 & 0.5403027956792810\end{array}$

$\begin{array}{lllll}0.2340184666322368 & 0.6469000740469856 & 0.0821215534931216\end{array}$

$\begin{array}{llllll}0.7340184560425873 & 0.6469000296943671 & 0.0821215618617327\end{array}$

$\begin{array}{lllll}0.2333300900861571 & 0.1469006802574446 & 0.2292174423948819\end{array}$

$\begin{array}{lllll}0.7333301652919461 & 0.1469006976693831 & 0.2292174296774489\end{array}$

$\begin{array}{lllll}0.2339143362926507 & 0.6468587899156462 & 0.3760855821453052\end{array}$

$\begin{array}{llllll}0.7339141642460596 & 0.6468588917773850 & 0.3760855889251584\end{array}$

$\begin{array}{llllll}0.2336567875851130 & 0.1476476304837124 & 0.5228517643516258\end{array}$

$\begin{array}{lllll}0.7336569755534106 & 0.1476488015346831 & 0.5228520989976342\end{array}$

$\begin{array}{lllll}0.3520019143123079 & 0.4663496737120351 & 0.0288387989009772\end{array}$

$\begin{array}{lllll}0.8520018838535633 & 0.4663496344114518 & 0.0288388017168824\end{array}$

$\begin{array}{lllll}0.3525214047662243 & 0.9667210493595101 & 0.1762041498671059\end{array}$

$\begin{array}{llllll}0.8525214413503596 & 0.9667210082280674 & 0.1762041501404420\end{array}$

$\begin{array}{llllll}0.3523690355989419 & 0.4665895135170272 & 0.3229854196533954\end{array}$

$\begin{array}{lllll}0.8523689694985508 & 0.4665895355478150 & 0.3229854466755956\end{array}$

$\begin{array}{lllll}0.3523807778544619 & 0.9671493333190352 & 0.4702200192175683\end{array}$

$\begin{array}{lllll}0.8523808613635181 & 0.9671492699695380 & 0.4702203668886131\end{array}$

$\begin{array}{lllll}0.2339945682997840 & 0.3521668481428042 & 0.0463430916112329\end{array}$

$\begin{array}{lllll}0.7339945160128465 & 0.3521668312436661 & 0.0463430813695831\end{array}$

$\begin{array}{llllll}0.2337548055114388 & 0.8529835874803680 & 0.1928572627674411\end{array}$

$\begin{array}{lllll}0.7337548453664967 & 0.8529835600268477 & 0.1928572810350586\end{array}$

$\begin{array}{llllll}0.2332327649635889 & 0.3529687422586640 & 0.3397154364320327\end{array}$

$\begin{array}{llllll}0.7332332080650348 & 0.3529684226515770 & 0.3397155375124365\end{array}$

$\begin{array}{lllll}0.2342474231510855 & 0.8531827564947539 & 0.4868468742009737\end{array}$

$\begin{array}{lllll}0.7342486178894193 & 0.8531846580812494 & 0.4868475867083626\end{array}$

$\begin{array}{lllll}0.1461051101018012 & 0.3823112504456632 & 0.0734870252970847\end{array}$

$\begin{array}{lllll}0.6461050690766184 & 0.3823112118864588 & 0.0734870201911886\end{array}$

$\begin{array}{lllll}0.1478941331292458 & 0.8808003698524861 & 0.2208351979623555\end{array}$

$\begin{array}{llllll}0.6478942018009158 & 0.8808003482177966 & 0.2208352068193531\end{array}$

$\begin{array}{lllll}0.1474402427783306 & 0.3811756256215930 & 0.3676653577956029\end{array}$

$\begin{array}{lllll}0.6474398092469529 & 0.3811756629924384 & 0.3676652631340567\end{array}$

$\begin{array}{llll}0.1475193479807193 & 0.8809039110549295 & 0.5146246818564809\end{array}$

$\begin{array}{lllll}0.6475208788648922 & 0.8809043297431548 & 0.5146259312472657\end{array}$

$\begin{array}{lllll}0.2663064548393160 & 0.4383125708077796 & 0.1071840237795752\end{array}$

$\begin{array}{llllll}0.7663064557070979 & 0.4383125155903927 & 0.1071840320275677\end{array}$

$\begin{array}{lllll}0.2663462453930555 & 0.9384498271586639 & 0.2542913960297041\end{array}$

$\begin{array}{lllll}0.7663462802934641 & 0.9384498312156115 & 0.2542914091828471\end{array}$

$\begin{array}{lllll}0.2664180746463483 & 0.4378914609097578 & 0.4010444252880700\end{array}$

$\begin{array}{llll}0.7664184498307766 & 0.4378919219792575 & 0.4010448277758243\end{array}$ 
$\begin{array}{llll}0.2661804481750601 & 0.9376881319286711 & 0.5482772503167619\end{array}$ $\begin{array}{llll}0.7661811160763721 & 0.9376880477975068 & 0.5482781446204767\end{array}$ $\begin{array}{llll}0.1476259689821247 & 0.6187450880481281 & 0.0542869799326272\end{array}$ $\begin{array}{llll}0.6476259308943507 & 0.6187450407775424 & 0.0542869935983143\end{array}$ $\begin{array}{lllll}0.1476249202433458 & 0.1187988889530527 & 0.2012333328707690\end{array}$ $\begin{array}{llll}0.6476249711297494 & 0.1187988533287065 & 0.2012333343643850\end{array}$ $\begin{array}{llll}0.1476776601872050 & 0.6189346967366076 & 0.3482276160697304\end{array}$ $\begin{array}{lllll}0.6476776245110901 & 0.6189346620142031 & 0.3482276064202481\end{array}$ $\begin{array}{llll}0.1459156446072397 & 0.1175045782381140 & 0.4956732564977565\end{array}$ $\begin{array}{llll}0.6459160164561141 & 0.1175039682400583 & 0.4956733532789561\end{array}$ $\begin{array}{llll}0.2662155265649926 & 0.5619754503348288 & 0.0208494871157298\end{array}$ $\begin{array}{llll}0.7662155178482606 & 0.5619754389030693 & 0.0208494964203789\end{array}$ $\begin{array}{llll}0.2664829442054488 & 0.0615913794282266 & 0.1678644037637324\end{array}$ $\begin{array}{llll}0.7664829923873121 & 0.0615913280051040 & 0.1678644167640642\end{array}$ $\begin{array}{llll}0.2664899014487133 & 0.5617082791101200 & 0.3146967441223015\end{array}$ $\begin{array}{llll}0.7664897811420037 & 0.5617081875973656 & 0.3146968094495295\end{array}$ $\begin{array}{llll}0.2662766404707087 & 0.0620225022437157 & 0.4620168449743838\end{array}$ $\begin{array}{lllll}0.7662767508288619 & 0.0620221634511250 & 0.4620171523978626\end{array}$ $\begin{array}{llll}0.3975539693644208 & 0.8811305944876711 & 0.0543015479874964\end{array}$ $\begin{array}{llll}0.8975538267078490 & 0.8811305791229326 & 0.0543015458930659\end{array}$ $\begin{array}{llll}0.3976727733490080 & 0.3810704304153156 & 0.2012419132744136\end{array}$ $\begin{array}{llll}0.8976728186105298 & 0.3810704212064036 & 0.2012419178634548\end{array}$ $\begin{array}{llll}0.3975994134187256 & 0.8809120498730783 & 0.3482127370711886\end{array}$ $\begin{array}{llll}0.8975994433631798 & 0.8809120176848749 & 0.3482127156442371\end{array}$ $\begin{array}{lllll}0.3959354539281470 & 0.3824124315439096 & 0.4956760678370684\end{array}$ $\begin{array}{lllll}0.8959355179719416 & 0.3824123406488604 & 0.4956760378661194\end{array}$ $\begin{array}{llll}0.5162016478387443 & 0.9378636769292747 & 0.0208600082355720\end{array}$ $\begin{array}{llll}0.0162015840840302 & 0.9378645300817123 & 0.0208599357904842\end{array}$ $\begin{array}{llll}0.5164945196251652 & 0.4383237540046629 & 0.1678652618462212\end{array}$ $\begin{array}{llll}0.0164945863031414 & 0.4383237361498212 & 0.1678652679446705\end{array}$ $\begin{array}{llll}0.5164590948918225 & 0.9381725387008456 & 0.3146926195060024\end{array}$ $\begin{array}{llll}0.0164591081531049 & 0.9381725183990004 & 0.3146926084343983\end{array}$ $\begin{array}{llll}0.5163124501993123 & 0.4378574632028704 & 0.4620159401457518\end{array}$ $\begin{array}{llll}0.0163125135773237 & 0.4378573586004028 & 0.4620159003459981\end{array}$ $\begin{array}{llll}0.3961499632874582 & 0.1175487301593893 & 0.0734925346750194\end{array}$ $\begin{array}{llll}0.8961497291939411 & 0.1175488544320785 & 0.0734925643482373\end{array}$ $\begin{array}{lllll}0.3979367968375125 & 0.6190862233794029 & 0.2208482916321365\end{array}$ $\begin{array}{lllll}0.8979368130123569 & 0.6190861938601024 & 0.2208482939647398\end{array}$ $\begin{array}{llll}0.3974465810982297 & 0.1186840085422013 & 0.3676674065319121\end{array}$ $\begin{array}{llll}0.8974466270994699 & 0.1186840159378550 & 0.3676674151173286\end{array}$ $\begin{array}{llll}0.3975593087482822 & 0.6190344863334435 & 0.5146170828717834\end{array}$ $\begin{array}{llll}0.8975594275271023 & 0.6190343882563306 & 0.5146170877629435\end{array}$ $\begin{array}{llll}0.5162998581948071 & 0.0615049354496354 & 0.1072045828659402\end{array}$ $\begin{array}{lllll}0.0162997528121971 & 0.0615049046072629 & 0.1072045875565756\end{array}$ $\begin{array}{llll}0.5163311483272505 & 0.5614828151902015 & 0.2542925518769624\end{array}$ $\begin{array}{lllll}0.0163310695197932 & 0.5614828370069654 & 0.2542925605595953\end{array}$ $\begin{array}{llll}0.5163566832203748 & 0.0619362180885628 & 0.4010426196952612\end{array}$ $\begin{array}{llll}0.0163567203141839 & 0.0619362239570079 & 0.4010426028519102\end{array}$ $\begin{array}{llll}0.5161990945628380 & 0.5622679121721255 & 0.5482724906032334\end{array}$ $\begin{array}{llll}0.0161992189397888 & 0.5622678542430500 & 0.5482724442333644\end{array}$ $\begin{array}{llll}0.6024327895109030 & 0.9667133301670323 & 0.0989234834481358\end{array}$ $\begin{array}{llll}0.1024326703935711 & 0.9667133164172940 & 0.0989234459296045\end{array}$ $\begin{array}{llll}0.6023192894956156 & 0.4664917040693988 & 0.2459786345017223\end{array}$ $\begin{array}{llll}0.1023193756796822 & 0.4664918301165648 & 0.2459786167169843\end{array}$ $\begin{array}{llll}0.6023849402149679 & 0.9669549173614066 & 0.3927948306500769\end{array}$ $\begin{array}{llll}0.1023849825531763 & 0.9669549360632438 & 0.3927948106193536\end{array}$ 
$\begin{array}{llll}0.6018450877176368 & 0.4664210461874670 & 0.5402996734057889\end{array}$ $\begin{array}{llll}0.1018452708386938 & 0.4664210432927698 & 0.5402996389466005\end{array}$ $\begin{array}{llll}0.4839355178300777 & 0.8529397692893590 & 0.0821356069866994\end{array}$ $\begin{array}{llll}0.9839353662592912 & 0.8529398645141767 & 0.0821356202904896\end{array}$ $\begin{array}{lllll}0.4833831631357366 & 0.3530076073317014 & 0.2292271236580103\end{array}$ $\begin{array}{llll}0.9833832277283783 & 0.3530075863035669 & 0.2292271238092156\end{array}$ $\begin{array}{llll}0.4838062029167108 & 0.8529434717900823 & 0.3760742608915148\end{array}$ $\begin{array}{llll}0.9838061700138833 & 0.8529434945941686 & 0.3760742672692451\end{array}$ $\begin{array}{llll}0.4836360245912041 & 0.3523103717302603 & 0.5228640023076706\end{array}$ $\begin{array}{llll}0.9836360585600806 & 0.3523103116217166 & 0.5228639839151275\end{array}$ $\begin{array}{llll}0.6019745106692925 & 0.0335042414134573 & 0.0288523732874474\end{array}$ $\begin{array}{llll}0.1019747622792164 & 0.0335052243838086 & 0.0288522861094239\end{array}$ $\begin{array}{llll}0.6025265247162143 & 0.5332182940176550 & 0.1761982471335079\end{array}$ $\begin{array}{llll}0.1025265595818300 & 0.5332182841705426 & 0.1761982496632173\end{array}$ $\begin{array}{llll}0.6022830589462971 & 0.0333385609294437 & 0.3229833940439353\end{array}$ $\begin{array}{lllll}0.1022830632856198 & 0.0333385492764797 & 0.3229833835800255\end{array}$ $\begin{array}{lllll}0.6024072752557857 & 0.5327852385588088 & 0.4702113911907105\end{array}$ $\begin{array}{llll}0.1024072825130101 & 0.5327852381598975 & 0.4702113499247050\end{array}$ $\begin{array}{llll}0.4840350317894250 & 0.1476458914336590 & 0.0463466658899182\end{array}$ $\begin{array}{llll}0.9840348455745515 & 0.1476459586442211 & 0.0463466643919965\end{array}$ $\begin{array}{llll}0.4837649090225613 & 0.6469167045142694 & 0.1928654102788603\end{array}$ $\begin{array}{llll}0.9837649386567685 & 0.6469166933679019 & 0.1928654221177121\end{array}$ $\begin{array}{llll}0.4833004369957178 & 0.1468905547373892 & 0.3397354501579137\end{array}$ $\begin{array}{llll}0.9833004631456357 & 0.1468905646227348 & 0.3397354495254648\end{array}$ $\begin{array}{llll}0.4843118789892368 & 0.6467464499553146 & 0.4868473920080437\end{array}$ $\begin{array}{llll}0.9843120270816649 & 0.6467464395821952 & 0.4868473996752514\end{array}$ $\begin{array}{llll}0.1875153135877250 & 0.4108820760779546 & 0.0550058689459394\end{array}$ $\begin{array}{llll}0.6875152786399499 & 0.4108820463946185 & 0.0550058686982939\end{array}$ $\begin{array}{llll}0.1875072622964387 & 0.9106623784310820 & 0.2021823315384422\end{array}$ $\begin{array}{llll}0.6875073253013326 & 0.9106623781674485 & 0.2021823449517352\end{array}$ $\begin{array}{llll}0.1871481729848179 & 0.4108437024074745 & 0.3490069184534104\end{array}$ $\begin{array}{llll}0.6871491284709255 & 0.4108435577403867 & 0.3490069987085636\end{array}$ $\begin{array}{lllll}0.1877630000721817 & 0.9108293536031652 & 0.4961007864688158\end{array}$ $\begin{array}{llll}0.6877601217765278 & 0.9108312456266072 & 0.4961010288981023\end{array}$ $\begin{array}{llll}0.1876961896440863 & 0.5890946626264425 & 0.0728870103702232\end{array}$ $\begin{array}{lllll}0.6876961596129786 & 0.5890946100223554 & 0.0728870224976948\end{array}$ $\begin{array}{lllll}0.1872218921414376 & 0.0890812312313188 & 0.2199031834441735\end{array}$ $\begin{array}{lllll}0.6872219776058603 & 0.0890812238779947 & 0.2199031842645914\end{array}$ $\begin{array}{lllll}0.1875534408590747 & 0.5891489215741647 & 0.3668331372055595\end{array}$ $\begin{array}{lllll}0.6875535104968935 & 0.5891489232594407 & 0.3668331956636047\end{array}$ $\begin{array}{lllll}0.1872335136423283 & 0.0889139333327776 & 0.5141599986022553\end{array}$ $\begin{array}{llll}0.6872338569008848 & 0.0889145490747630 & 0.5141603216868220\end{array}$ $\begin{array}{llll}0.3126886018174501 & 0.4696882300673798 & 0.0901948141379579\end{array}$ $\begin{array}{lllll}0.8126885952250191 & 0.4696881921989465 & 0.0901948208139238\end{array}$ $\begin{array}{llll}0.3125287146838416 & 0.9699580778102173 & 0.2372931961239421\end{array}$ $\begin{array}{llll}0.8125287511569282 & 0.9699581061473013 & 0.2372932156396961\end{array}$ $\begin{array}{llll}0.3126045721320467 & 0.4694908708189700 & 0.3840535905860930\end{array}$ $\begin{array}{llll}0.8126044269538203 & 0.4694913526828353 & 0.3840535965994298\end{array}$ $\begin{array}{llll}0.3126455514718233 & 0.9698956293821804 & 0.5314251409576637\end{array}$ $\begin{array}{llll}0.8126444347933001 & 0.9698950244121891 & 0.5314255899046492\end{array}$ $\begin{array}{llll}0.2502567263252847 & 0.4998909799853887 & 0.0639753995875129\end{array}$ $\begin{array}{llll}0.7502566978402890 & 0.4998909218462723 & 0.0639754025379565\end{array}$ $\begin{array}{llll}0.2499499998348066 & 0.9999518171076205 & 0.2110673340175504\end{array}$ $\begin{array}{llll}0.7499500834266537 & 0.9999518110571894 & 0.2110673464267681\end{array}$ $\begin{array}{llll}0.2499390556771727 & 0.4998905612324198 & 0.3578812010541187\end{array}$ $\begin{array}{llll}0.7499400954904797 & 0.4998907397493081 & 0.3578816833118608\end{array}$ 
$\begin{array}{llll}0.2501185856403009 & 0.0000492418603632 & 0.5051726454346712\end{array}$ $\begin{array}{llll}0.7501190986118610 & 0.0000482332234142 & 0.5051734111459505\end{array}$ $\begin{array}{llll}0.3127800717151784 & 0.5299755113562448 & 0.0377037992580518\end{array}$ $\begin{array}{llll}0.8127800386374503 & 0.5299754604657380 & 0.0377038063042235\end{array}$ $\begin{array}{llll}0.3126373238947920 & 0.0301520500386520 & 0.1848827063022079\end{array}$ $\begin{array}{llll}0.8126373954419343 & 0.0301520064704488 & 0.1848827149184331\end{array}$ $\begin{array}{llll}0.3126080892548841 & 0.5300725684328711 & 0.3316916601013548\end{array}$ $\begin{array}{llll}0.8126077451625762 & 0.5300733041831832 & 0.3316911785704105\end{array}$ $\begin{array}{llll}0.3126804327074460 & 0.0305616762134924 & 0.4789836970296180\end{array}$ $\begin{array}{llll}0.8126814320462149 & 0.0305616863882773 & 0.4789839081614926\end{array}$ $\begin{array}{llll}0.4376437956112352 & 0.9107578908413735 & 0.0729014660595336\end{array}$ $\begin{array}{llll}0.9376437704982720 & 0.9107580953920706 & 0.0729014320912339\end{array}$ $\begin{array}{llll}0.4372714857916069 & 0.4108014711483294 & 0.2199097990337039\end{array}$ $\begin{array}{llll}0.9372715134963500 & 0.4108014315701885 & 0.2199098054303357\end{array}$ $\begin{array}{llll}0.4374771054049535 & 0.9106919708553645 & 0.3668222981773024\end{array}$ $\begin{array}{llll}0.9374770863615407 & 0.9106919778958376 & 0.3668222866356333\end{array}$ $\begin{array}{llll}0.4372427577614498 & 0.4110203320649941 & 0.5141597373293825\end{array}$ $\begin{array}{llll}0.9372428465370848 & 0.4110202549226074 & 0.5141597016938142\end{array}$ $\begin{array}{llll}0.4375371377388130 & 0.0889486070295322 & 0.0550136016225163\end{array}$ $\begin{array}{llll}0.9375368847417620 & 0.0889488176882510 & 0.0550136038238691\end{array}$ $\begin{array}{llll}0.4375127082448194 & 0.5892419432081396 & 0.2021922881567416\end{array}$ $\begin{array}{llll}0.9375127122805694 & 0.5892419138851601 & 0.2021922806994199\end{array}$ $\begin{array}{llll}0.4371568836867539 & 0.0890253470793144 & 0.3490117574377740\end{array}$ $\begin{array}{llll}0.9371569035058126 & 0.0890253660421803 & 0.3490117575006016\end{array}$ $\begin{array}{lllll}0.4377927207735808 & 0.5890943093931021 & 0.4960925506936523\end{array}$ $\begin{array}{lllll}0.9377928608896634 & 0.5890942822241577 & 0.4960925501807177\end{array}$ $\begin{array}{lllll}0.5626731200933114 & 0.0301525216506657 & 0.0902072924146478\end{array}$ $\begin{array}{llll}0.0626730059397628 & 0.0301525902883290 & 0.0902072787892489\end{array}$ $\begin{array}{llll}0.5625457094732531 & 0.5299931676957041 & 0.2372911024860280\end{array}$ $\begin{array}{llll}0.0625455226611029 & 0.5299931449925347 & 0.2372911118784445\end{array}$ $\begin{array}{llll}0.5625504622681826 & 0.0303491827151365 & 0.3840546719264917\end{array}$ $\begin{array}{llll}0.0625504839317209 & 0.0303491875100403 & 0.3840546526839544\end{array}$ $\begin{array}{llll}0.5626704375279911 & 0.5300685446763260 & 0.5314262245983531\end{array}$ $\begin{array}{llll}0.0626705597951801 & 0.5300684725194510 & 0.5314261773712861\end{array}$ $\begin{array}{llll}0.5002331960095457 & 0.9999495326461716 & 0.0639826171224459\end{array}$ $\begin{array}{llll}0.0002330720639433 & 0.9999498226972108 & 0.0639825952406213\end{array}$ $\begin{array}{llll}0.4999694756178390 & 0.4999580124397781 & 0.2110699891837914\end{array}$ $\begin{array}{lllll}-0.0000305352026705 & 0.4999579626671050 & 0.2110699792524592\end{array}$ $\begin{array}{llll}0.4998948562855660 & 0.9999446295006579 & 0.3578826558072065\end{array}$ $\begin{array}{llll}0.9998948664711200 & 0.9999446352146073 & 0.3578826451424756\end{array}$ $\begin{array}{llll}0.5001376363448148 & 0.4998703302149403 & 0.5051668334365967\end{array}$ $\begin{array}{llll}0.0001377353662219 & 0.4998702234156639 & 0.5051668044641902\end{array}$ $\begin{array}{llll}0.5627551331271929 & 0.9698592557430805 & 0.0377158721004725\end{array}$ $\begin{array}{llll}0.0627556504511697 & 0.9698601305950509 & 0.0377157809401695\end{array}$ $\begin{array}{llll}0.5626630812303787 & 0.4697811851821405 & 0.1848843899786165\end{array}$ $\begin{array}{llll}0.0626631418874365 & 0.4697811753812982 & 0.1848843934094380\end{array}$ $\begin{array}{llll}0.5625596017782567 & 0.9698277827560485 & 0.3316910939289539\end{array}$ $\begin{array}{llll}0.0625596136897880 & 0.9698277706882354 & 0.3316910810655584\end{array}$ $\begin{array}{lllll}0.5626972122314551 & 0.4693692043311645 & 0.4789850120078570\end{array}$ $\begin{array}{llll}0.0626972974408433 & 0.4693691385380181 & 0.4789849656934875\end{array}$ $\begin{array}{llll}0.0649889810247451 & 0.3648156431299922 & 0.0195732057784048\end{array}$ $\begin{array}{llll}0.5649889106713726 & 0.3648157180682536 & 0.0195732034773790\end{array}$ $\begin{array}{lllll}0.0652802693977015 & 0.8649327228171397 & 0.1669677964692577\end{array}$ $\begin{array}{llll}0.5652803288714926 & 0.8649327644010830 & 0.1669678025383066\end{array}$ $\begin{array}{llll}0.0647327726991954 & 0.3649166071259818 & 0.3138928728512717\end{array}$ $\begin{array}{llll}0.5647332107521863 & 0.3649162651217136 & 0.3138927169846527\end{array}$ 
$\begin{array}{llll}0.0657508751014087 & 0.8652086086819849 & 0.4607876908551891\end{array}$ $\begin{array}{llll}0.5657509546502477 & 0.8652092815756274 & 0.4607880774689608\end{array}$ $\begin{array}{lllll}0.0655773565778823 & 0.6349563075188316 & 0.1081352829144975\end{array}$ $\begin{array}{llll}0.5655773624614885 & 0.6349562402815765 & 0.1081353021974733\end{array}$ $\begin{array}{llll}0.0648692623445778 & 0.1350183146923691 & 0.2550377317023775\end{array}$ $\begin{array}{llll}0.5648693431726415 & 0.1350183308471588 & 0.2550377345642573\end{array}$ $\begin{array}{llll}0.0654269428489188 & 0.6349215857055613 & 0.4020754181808839\end{array}$ $\begin{array}{llll}0.5654268422613870 & 0.6349216511854050 & 0.4020754250724650\end{array}$ $\begin{array}{llll}0.0647402058427898 & 0.1351273041245902 & 0.5495739537729941\end{array}$ $\begin{array}{lllll}0.5647402488800963 & 0.1351270451959411 & 0.5495739767891412\end{array}$ $\begin{array}{llll}0.4347029916491096 & 0.3498349223696655 & 0.1037953930358895\end{array}$ $\begin{array}{llll}0.9347030078096049 & 0.3498349247347377 & 0.1037954038539234\end{array}$ $\begin{array}{llll}0.4344689091592239 & 0.8498353397723846 & 0.2507651231291267\end{array}$ $\begin{array}{llll}0.9344688731932612 & 0.8498353444408088 & 0.2507651155166399\end{array}$ $\begin{array}{lllll}0.4348687328816301 & 0.3497472672546709 & 0.3974733893372767\end{array}$ $\begin{array}{lllll}0.9348689541809686 & 0.3497473425747411 & 0.3974734695027039\end{array}$ $\begin{array}{lllll}0.4343841725906699 & 0.8497727430517876 & 0.5452877074009402\end{array}$ $\begin{array}{lllll}0.9343846313490134 & 0.8497719841137777 & 0.5452878210079832\end{array}$ $\begin{array}{llll}0.4345074027613522 & 0.6499780262160633 & 0.0237432974218708\end{array}$ $\begin{array}{llll}0.9345073780095546 & 0.6499780360616962 & 0.0237433250844367\end{array}$ $\begin{array}{llll}0.4348194182836945 & 0.1500930657108995 & 0.1714562586217839\end{array}$ $\begin{array}{lllll}0.9348194103002090 & 0.1500930780392535 & 0.1714562539316907\end{array}$ $\begin{array}{llll}0.4346786199920053 & 0.6501991600140117 & 0.3183233252965635\end{array}$ $\begin{array}{lllll}0.9346785492600004 & 0.6501990859124456 & 0.3183233322752272\end{array}$ $\begin{array}{lllll}0.4348636762636633 & 0.1501219207106007 & 0.4653402913829321\end{array}$ $\begin{array}{llll}0.9348637693918002 & 0.1501219589672934 & 0.4653403195332823\end{array}$ $\begin{array}{llll}0.6847018999536987 & 0.1500151140916210 & 0.1038164420429250\end{array}$ $\begin{array}{llll}0.1847017669757265 & 0.1500150826362949 & 0.1038164304049964\end{array}$ $\begin{array}{llll}0.6845466837755739 & 0.6500624217249329 & 0.2507763222302308\end{array}$ $\begin{array}{llll}0.1845480523184115 & 0.6500609476772371 & 0.2507763502211592\end{array}$ $\begin{array}{llll}0.6848341938341582 & 0.1500908904733576 & 0.3974767698577510\end{array}$ $\begin{array}{llll}0.1848342703511479 & 0.1500908386913145 & 0.3974767352504515\end{array}$ $\begin{array}{llll}0.6844225058957782 & 0.6502097879173739 & 0.5452799614060241\end{array}$ $\begin{array}{llll}0.1844225661288011 & 0.6502098720483503 & 0.5452798420969717\end{array}$ $\begin{array}{llll}0.6844192458606184 & 0.8498507892922443 & 0.0237428335587516\end{array}$ $\begin{array}{llll}0.1844189722621311 & 0.8498510729708922 & 0.0237427728110530\end{array}$ $\begin{array}{llll}0.6847659745218690 & 0.3497956025566777 & 0.1714668500947305\end{array}$ $\begin{array}{llll}0.1847659945866820 & 0.3497955552686299 & 0.1714668358646010\end{array}$ $\begin{array}{llll}0.6846689639771982 & 0.8497529366248379 & 0.3183043512414759\end{array}$ $\begin{array}{llll}0.1846690359442884 & 0.8497529569954947 & 0.3183043582041978\end{array}$ $\begin{array}{llll}0.6848416169732648 & 0.3497227120384143 & 0.4653611110547932\end{array}$ $\begin{array}{llll}0.1848417475277779 & 0.3497227204880852 & 0.4653610697846863\end{array}$ $\begin{array}{llll}0.3156068132566163 & 0.8649001506472762 & 0.1081721922484601\end{array}$ $\begin{array}{llll}0.8156066867412590 & 0.8649002392529738 & 0.1081721926370899\end{array}$ $\begin{array}{llll}0.3148554786534339 & 0.3649138754708283 & 0.2550373240970238\end{array}$ $\begin{array}{lllll}0.8148555000334813 & 0.3649138710335117 & 0.2550373332043222\end{array}$ $\begin{array}{lllll}0.3154307962042706 & 0.8649171478355927 & 0.4020963561025088\end{array}$ $\begin{array}{lllll}0.8154306987825661 & 0.8649171775957157 & 0.4020963216021031\end{array}$ $\begin{array}{lllll}0.3147593156683435 & 0.3648347228811616 & 0.5495888117716671\end{array}$ $\begin{array}{llll}0.8147594631268936 & 0.3648346837580962 & 0.5495888099120548\end{array}$ $\begin{array}{lllll}0.3150222462633854 & 0.1350335059834988 & 0.0195708029140394\end{array}$ $\begin{array}{llll}0.8150221361174343 & 0.1350335202762672 & 0.0195707828901923\end{array}$ $\begin{array}{llll}0.3152948657039039 & 0.6350094631538338 & 0.1669823892015617\end{array}$ $\begin{array}{lllll}0.8152949478651358 & 0.6350094474638667 & 0.1669823676260701\end{array}$ $\begin{array}{llll}0.3147562205818185 & 0.1350737639416931 & 0.3139086949565939\end{array}$ $\begin{array}{llll}0.8147562650404898 & 0.1350738598574466 & 0.3139087001258449\end{array}$ 
$\begin{array}{llll}0.3157564021460077 & 0.6347327474311291 & 0.4607894124533435\end{array}$ $\begin{array}{lllll}0.8157564376825029 & 0.6347327107503914 & 0.4607894259075517\end{array}$ $\begin{array}{lllll}0.0878922417856410 & 0.2815728937590927 & 0.0262202211863416\end{array}$ $\begin{array}{llll}0.5878920793120888 & 0.2815729470062142 & 0.0262202287309015\end{array}$ $\begin{array}{llll}0.0880309198176239 & 0.7816167139979939 & 0.1734718492450758\end{array}$ $\begin{array}{llll}0.5880308940309954 & 0.7816167315072592 & 0.1734718692454257\end{array}$ $\begin{array}{llll}0.0875263286415119 & 0.2816705345382775 & 0.3204590916478057\end{array}$ $\begin{array}{llll}0.5875259380185573 & 0.2816700048129165 & 0.3204591177035460\end{array}$ $\begin{array}{lllll}0.0882095492583365 & 0.7818738463889219 & 0.4673551076563086\end{array}$ $\begin{array}{llll}0.5882090595687869 & 0.7818742186771918 & 0.4673561443324378\end{array}$ $\begin{array}{llll}0.1196840977204742 & 0.4446502651331066 & 0.0331411189174294\end{array}$ $\begin{array}{llll}0.6196840659840978 & 0.4446502972540506 & 0.0331411234332352\end{array}$ $\begin{array}{llll}0.1173413363288711 & 0.9444428284026122 & 0.1809343140852739\end{array}$ $\begin{array}{llll}0.6173414286510928 & 0.9444428444190527 & 0.1809343229998834\end{array}$ $\begin{array}{lllll}0.1170569207261363 & 0.4445776682717896 & 0.3277302783273247\end{array}$ $\begin{array}{llll}0.6170594217821633 & 0.4445772710751147 & 0.3277296026275276\end{array}$ $\begin{array}{llll}0.1182170040644720 & 0.9447054132045749 & 0.4746939629425602\end{array}$ $\begin{array}{llll}0.6182177442748183 & 0.9447050986043508 & 0.4746947356402770\end{array}$ $\begin{array}{llll}0.0029203806574906 & 0.3951799842929391 & 0.0031187113057107\end{array}$ $\begin{array}{lllll}0.5029203576799711 & 0.3951801037072371 & 0.0031186930665474\end{array}$ $\begin{array}{llll}0.0038566198666712 & 0.8950499466093299 & 0.1501316105886450\end{array}$ $\begin{array}{llll}0.5038567755057294 & 0.8950500178010581 & 0.1501316034572728\end{array}$ $\begin{array}{llll}0.0030944543135410 & 0.3948433618275753 & 0.2970987404032610\end{array}$ $\begin{array}{llll}0.5030943916259282 & 0.3948432844467741 & 0.2970987894582600\end{array}$ $\begin{array}{lllll}0.0044136790556564 & 0.8954188218162658 & 0.4439470823470787\end{array}$ $\begin{array}{llll}0.5044168251051255 & 0.8954205263146772 & 0.4439472252260431\end{array}$ $\begin{array}{llll}0.0882996539848303 & 0.7182504774549892 & 0.1016071136429466\end{array}$ $\begin{array}{llll}0.5882996293449838 & 0.7182504096432000 & 0.1016071150985073\end{array}$ $\begin{array}{llll}0.0878207940384943 & 0.2182943731635318 & 0.2485436028611537\end{array}$ $\begin{array}{llll}0.5878208153049855 & 0.2182943802156241 & 0.2485435840632929\end{array}$ $\begin{array}{llll}0.0880388755841792 & 0.7182377167493017 & 0.3955268710421166\end{array}$ $\begin{array}{llll}0.5880388639179400 & 0.7182378012534741 & 0.3955269038969008\end{array}$ $\begin{array}{llll}0.0877859945350896 & 0.2183606760894120 & 0.5429532909401429\end{array}$ $\begin{array}{llll}0.5877860584623292 & 0.2183603757165589 & 0.5429532432048537\end{array}$ $\begin{array}{llll}0.1179667275853158 & 0.5553592434860488 & 0.0942489348265895\end{array}$ $\begin{array}{llll}0.6179667414808346 & 0.5553591811506927 & 0.0942489517490863\end{array}$ $\begin{array}{lllll}0.1170448674874678 & 0.0553732235777714 & 0.2411536895962480\end{array}$ $\begin{array}{lllll}0.6170450000538444 & 0.0553732282279622 & 0.2411537035854741\end{array}$ $\begin{array}{llll}0.1177438762965097 & 0.5554027358395366 & 0.3881848862357756\end{array}$ $\begin{array}{llll}0.6177437217845241 & 0.5554027651980999 & 0.3881848798678518\end{array}$ $\begin{array}{llll}0.1192951312901548 & 0.0551568237772788 & 0.5359957494412919\end{array}$ $\begin{array}{llll}0.6192958190426325 & 0.0551568487668492 & 0.5359959791742505\end{array}$ $\begin{array}{llll}0.0040139445523377 & 0.6048879996421026 & 0.1249241267712949\end{array}$ $\begin{array}{llll}0.5040140009274120 & 0.6048879485468719 & 0.1249241601628540\end{array}$ $\begin{array}{lllll}0.0031642430740936 & 0.1050259769804357 & 0.2717982651929297\end{array}$ $\begin{array}{lllll}0.5031643774590002 & 0.1050259924108971 & 0.2717982784133662\end{array}$ $\begin{array}{llll}0.0039957930272132 & 0.6047690057540889 & 0.4188988658893157\end{array}$ $\begin{array}{llll}0.5039955815427940 & 0.6047690534114514 & 0.4188988635100934\end{array}$ $\begin{array}{llll}0.0025957379843690 & 0.1048115608657266 & 0.5660163634497325\end{array}$ $\begin{array}{lllll}0.5025954102684456 & 0.1048115446430604 & 0.5660163073803984\end{array}$ $\begin{array}{lllll}0.4119859107721778 & 0.3723156633529102 & 0.1282521473883242\end{array}$ $\begin{array}{llll}0.9119858674903245 & 0.3723156136692534 & 0.1282521569687111\end{array}$ $\begin{array}{llll}0.4116887886826058 & 0.8719144365427800 & 0.2752479199871559\end{array}$ $\begin{array}{llll}0.9116888202629223 & 0.8719143609145479 & 0.2752479321649052\end{array}$ $\begin{array}{llll}0.4120651717908188 & 0.3718431977329470 & 0.4219483990129369\end{array}$ $\begin{array}{llll}0.9120654827332129 & 0.3718429468123622 & 0.4219484239436243\end{array}$ 
$\begin{array}{llll}0.4108422812154045 & 0.8715054247966421 & 0.5696613129239801\end{array}$ $\begin{array}{llll}0.9108434442884064 & 0.8715047810446037 & 0.5696614596423707\end{array}$ $\begin{array}{llll}0.3826401105850614 & 0.3971094943316224 & 0.0803678149520287\end{array}$ $\begin{array}{llll}0.8826401511523629 & 0.3971095122172730 & 0.0803678262651189\end{array}$ $\begin{array}{llll}0.3824759863330410 & 0.8974285160848731 & 0.2273972811417362\end{array}$ $\begin{array}{llll}0.8824760577080321 & 0.8974285658647221 & 0.2273973054741858\end{array}$ $\begin{array}{llll}0.3824907707049691 & 0.3969804104890632 & 0.3741093059802655\end{array}$ $\begin{array}{llll}0.8824909766933011 & 0.3969815150938427 & 0.3741097577843912\end{array}$ $\begin{array}{llll}0.3830647354692593 & 0.8979083267448139 & 0.5216432651307633\end{array}$ $\begin{array}{lllll}0.8830638290430164 & 0.8979075447139652 & 0.5216434793532407\end{array}$ $\begin{array}{lllll}0.4960609032988916 & 0.2924094030397366 & 0.0950022707899231\end{array}$ $\begin{array}{llll}0.9960609582586752 & 0.2924094536338272 & 0.0950022856199858\end{array}$ $\begin{array}{lllll}0.4958609362941881 & 0.7925406462000458 & 0.2419067897444561\end{array}$ $\begin{array}{llll}0.9958609734681590 & 0.7925407201923736 & 0.2419067893090847\end{array}$ $\begin{array}{llll}0.4965404769231772 & 0.2927533974540567 & 0.3886200391135092\end{array}$ $\begin{array}{llll}0.9965406653866729 & 0.2927532750066733 & 0.3886199905284662\end{array}$ $\begin{array}{llll}0.4959480927299212 & 0.7924756679443923 & 0.5364201080064269\end{array}$ $\begin{array}{llll}0.9959485457024502 & 0.7924748374280487 & 0.5364201757271992\end{array}$ $\begin{array}{lll}0.4111509627701133 & 0.6277251727876108 & -0.0006044529029540\end{array}$ $0.91115086110104120 .6277253121762248-0.0006044286625605$ $\begin{array}{llll}0.4120774909181509 & 0.1280079499378913 & 0.1469748347764534\end{array}$ $\begin{array}{llll}0.9120774234247514 & 0.1280080015897792 & 0.1469748264104040\end{array}$ $\begin{array}{lllll}0.4120823164139367 & 0.6280433218671881 & 0.2938339856970706\end{array}$ $\begin{array}{llll}0.9120821435246657 & 0.6280432930768233 & 0.2938339850647944\end{array}$ $\begin{array}{lllll}0.4118761890805150 & 0.1276538146799232 & 0.4409076820866776\end{array}$ $\begin{array}{llll}0.9118758180095369 & 0.1276533551077983 & 0.4409077415871422\end{array}$ $\begin{array}{llll}0.3831245553022080 & 0.6021458978070248 & 0.0474391450083503\end{array}$ $\begin{array}{llll}0.8831245478234065 & 0.6021458096925454 & 0.0474391652517490\end{array}$ $\begin{array}{llll}0.3824613220688265 & 0.1027742030914093 & 0.1948121070347519\end{array}$ $\begin{array}{llll}0.8824613996296924 & 0.1027741455484934 & 0.1948121024669458\end{array}$ $\begin{array}{llll}0.3825540395965822 & 0.6025453435588852 & 0.3416488075237740\end{array}$ $\begin{array}{llll}0.8825540167473922 & 0.6025454470159759 & 0.3416487883965310\end{array}$ $\begin{array}{llll}0.3826947459823400 & 0.1030890485051281 & 0.4888231899677858\end{array}$ $\begin{array}{lllll}0.8826958044795414 & 0.1030893248285184 & 0.4888232887269406\end{array}$ $\begin{array}{llll}0.4959262272286035 & 0.7075570254371133 & 0.0325454774740940\end{array}$ $\begin{array}{llll}0.9959262403847201 & 0.7075569586510084 & 0.0325455234172979\end{array}$ $\begin{array}{lllll}0.4964229961435994 & 0.2071591104861380 & 0.1803219196325946\end{array}$ $\begin{array}{llll}0.9964230030199439 & 0.2071591423079034 & 0.1803219088882449\end{array}$ $\begin{array}{llll}0.4959688231526298 & 0.7075863994922188 & 0.3272046862588465\end{array}$ $\begin{array}{llll}0.9959688956465351 & 0.7075862530555121 & 0.3272046596268621\end{array}$ $\begin{array}{llll}0.4965457893815475 & 0.2072923899089469 & 0.4740808602200761\end{array}$ $\begin{array}{lllll}0.9965457650380921 & 0.2072928403681008 & 0.4740807518088505\end{array}$ $\begin{array}{lllll}0.6620265888943617 & 0.1274312266867372 & 0.1282684846492960\end{array}$ $\begin{array}{lllll}0.1620265264145666 & 0.1274310857787426 & 0.1282684684836100\end{array}$ $\begin{array}{llll}0.6617981369626489 & 0.6278568211518935 & 0.2752522396953440\end{array}$ $\begin{array}{llll}0.1617975443626553 & 0.6278571366050728 & 0.2752521939348205\end{array}$ $\begin{array}{llll}0.6620362526402634 & 0.1279531087024756 & 0.4219506782888705\end{array}$ $\begin{array}{llll}0.1620363571097465 & 0.1279529880368796 & 0.4219506450507522\end{array}$ $\begin{array}{lllll}0.6609036358656717 & 0.6284232792698684 & 0.5696536027432981\end{array}$ $\begin{array}{llll}0.1609039228687554 & 0.6284232029113979 & 0.5696534947140219\end{array}$ $\begin{array}{llll}0.6326020873659665 & 0.1027791575492436 & 0.0803797763743414\end{array}$ $\begin{array}{llll}0.1326019698849857 & 0.1027792099591292 & 0.0803797799917139\end{array}$ $\begin{array}{llll}0.6324701301956491 & 0.6025696726500925 & 0.2274021662659098\end{array}$ $\begin{array}{llll}0.1324699035685712 & 0.6025697771293267 & 0.2274021764971298\end{array}$ $\begin{array}{llll}0.6324329211866835 & 0.1028830925796794 & 0.3741093024728570\end{array}$ $\begin{array}{llll}0.1324329181927331 & 0.1028830843839381 & 0.3741092660765581\end{array}$ 
$\begin{array}{llll}0.6330848688820105 & 0.6020748138247912 & 0.5216361244740265\end{array}$ $\begin{array}{llll}0.1330848604816532 & 0.6020747683955788 & 0.5216360261802447\end{array}$ $\begin{array}{llll}0.7460325146296963 & 0.2074847769033897 & 0.0950257376672975\end{array}$ $\begin{array}{llll}0.2460323212657893 & 0.2074848344023223 & 0.0950257253065472\end{array}$ $\begin{array}{llll}0.7459431743554076 & 0.7073801406570693 & 0.2419305335094163\end{array}$ $\begin{array}{llll}0.2459463344435665 & 0.7073771842101699 & 0.2419307512261406\end{array}$ $\begin{array}{llll}0.7465182162770656 & 0.2071117814209903 & 0.3886334102681553\end{array}$ $\begin{array}{llll}0.2465183121824864 & 0.2071117365154655 & 0.3886333751625593\end{array}$ $\begin{array}{llll}0.7459640364341158 & 0.7075432156403633 & 0.5364164446534334\end{array}$ $\begin{array}{llll}0.2459638258512729 & 0.7075435598859541 & 0.5364162812453848\end{array}$ $\begin{array}{llll}0.3383368326225734 & 0.7816233175608150 & 0.1016404862549105\end{array}$ $\begin{array}{llll}0.8383366770603836 & 0.7816234211033283 & 0.1016405059495115\end{array}$ $\begin{array}{llll}0.3377655719229795 & 0.2816262925807214 & 0.2485490625677356\end{array}$ $\begin{array}{llll}0.8377655986660705 & 0.2816262345099472 & 0.2485490419303932\end{array}$ $\begin{array}{llll}0.3380287884849255 & 0.7815995807682016 & 0.3955473314600955\end{array}$ $\begin{array}{llll}0.8380286906548519 & 0.7815996079750311 & 0.3955473041049710\end{array}$ $\begin{array}{llll}0.3378511281278563 & 0.2815974978602013 & 0.5429893782579376\end{array}$ $\begin{array}{llll}0.8378513859632991 & 0.2815974431719609 & 0.5429894202590488\end{array}$ $0.66103031287375880 .8720997410267041-0.0006035299054743$ $0.16103013978311140 .8720998311472840-0.0006036092329912$ $\begin{array}{llll}0.6619944001291658 & 0.3718558839190844 & 0.1469830886760239\end{array}$ $\begin{array}{llll}0.1619943332371129 & 0.3718558200820902 & 0.1469830775591195\end{array}$ $\begin{array}{lllll}0.6620731604263015 & 0.8719432739562107 & 0.2938180333739654\end{array}$ $\begin{array}{lllll}0.1620733416731746 & 0.8719433132404405 & 0.2938180342041798\end{array}$ $\begin{array}{llll}0.6617575256450511 & 0.3719968811850614 & 0.4409241955891205\end{array}$ $\begin{array}{llll}0.1617576918857224 & 0.3719968860233182 & 0.4409241494923216\end{array}$ $\begin{array}{llll}0.6331054547770547 & 0.8977051599917164 & 0.0474419898832959\end{array}$ $\begin{array}{llll}0.1331053158389576 & 0.8977059084357069 & 0.0474418933105172\end{array}$ $\begin{array}{llll}0.6324671316329030 & 0.3971944584248339 & 0.1948185778768632\end{array}$ $\begin{array}{llll}0.1324672225436689 & 0.3971944773667855 & 0.1948185778792634\end{array}$ $\begin{array}{llll}0.6325424207781439 & 0.8973778624035899 & 0.3416344297394748\end{array}$ $\begin{array}{lllll}0.1325424323975358 & 0.8973778704626284 & 0.3416344241197747\end{array}$ $\begin{array}{llll}0.6327346048980381 & 0.3968971591554702 & 0.4888319747889182\end{array}$ $\begin{array}{llll}0.1327347559926699 & 0.3968971773587819 & 0.4888319276554415\end{array}$ $\begin{array}{llll}0.7458104764904785 & 0.7922283760112285 & 0.0325413066680767\end{array}$ $\begin{array}{llll}0.2458099709812137 & 0.7922286271671888 & 0.0325412927581959\end{array}$ $\begin{array}{lllll}0.7463249389062687 & 0.2927179846282651 & 0.1803393267107752\end{array}$ $\begin{array}{llll}0.2463249633085338 & 0.2927179443243721 & 0.1803393041896198\end{array}$ $\begin{array}{llll}0.7459413417655130 & 0.7923704147892860 & 0.3271819160115346\end{array}$ $\begin{array}{llll}0.2459413838221010 & 0.7923704022621363 & 0.3271819381230334\end{array}$ $\begin{array}{llll}0.7465756124833209 & 0.2926454258566958 & 0.4741183381948070\end{array}$ $\begin{array}{llll}0.2465757205712582 & 0.2926454167862682 & 0.4741182915665887\end{array}$ $\begin{array}{llll}0.3679175960085744 & 0.9445139675769800 & 0.0942596272090094\end{array}$ $\begin{array}{llll}0.8679174926758128 & 0.9445141156934798 & 0.0942595936090824\end{array}$ $\begin{array}{llll}0.3670996927011262 & 0.4445404808246528 & 0.2411546138913134\end{array}$ $\begin{array}{llll}0.8670997881729525 & 0.4445404694779126 & 0.2411546237699747\end{array}$ $\begin{array}{llll}0.3676444771511954 & 0.9444250401568312 & 0.3881685711585863\end{array}$ $\begin{array}{llll}0.8676443880233800 & 0.9444250757978110 & 0.3881685359638395\end{array}$ $\begin{array}{lllll}0.3692946415804671 & 0.4447897394239900 & 0.5359895769847028\end{array}$ $\begin{array}{llll}0.8692946867265070 & 0.4447896809486798 & 0.5359895307308126\end{array}$ $\begin{array}{llll}0.2541053474263953 & 0.8949599457626117 & 0.1249817141498792\end{array}$ $\begin{array}{lllll}0.7541052100019866 & 0.8949600415951449 & 0.1249817207770087\end{array}$ $\begin{array}{llll}0.2531545130863049 & 0.3949378147049613 & 0.2717913146148792\end{array}$ $\begin{array}{llll}0.7531546348015798 & 0.3949377694794107 & 0.2717913258600549\end{array}$ $\begin{array}{llll}0.2540784387921116 & 0.8950474353751847 & 0.4189481842860798\end{array}$ $\begin{array}{llll}0.7540783634134035 & 0.8950474736674979 & 0.4189481615852274\end{array}$ 
$\begin{array}{llll}0.2526098228171233 & 0.3951577782403993 & 0.5660204944335292\end{array}$ $\begin{array}{lllll}0.7526099351061254 & 0.3951577555593676 & 0.5660204803279155\end{array}$ $\begin{array}{lllll}0.3379518849507295 & 0.2182821378392059 & 0.0262115281659437\end{array}$ $\begin{array}{lllll}0.8379518059133437 & 0.2182821544120930 & 0.0262115105444443\end{array}$ $\begin{array}{lllll}0.3380721898444168 & 0.7183051540895229 & 0.1735116767711941\end{array}$ $\begin{array}{lllll}0.83807228886638681 & 0.7183051795042628 & 0.1735116877247482\end{array}$ $\begin{array}{lllll}0.3375619433045737 & 0.2182957453462613 & 0.3204939634047771\end{array}$ $\begin{array}{lllll}0.8375621123123518 & 0.2182958202904722 & 0.3204939461357891\end{array}$ $\begin{array}{lllll}0.3382367902163769 & 0.7180712511790102 & 0.4673498891182722\end{array}$ $\begin{array}{lllll}0.8382367532126589 & 0.7180712374894027 & 0.4673499160569192\end{array}$ $\begin{array}{lllll}0.3697054329171819 & 0.0551778622758618 & 0.0331479846366848\end{array}$ $\begin{array}{llllll}0.8697052556908322 & 0.0551779020871864 & 0.0331480032165332\end{array}$ $\begin{array}{lllll}0.3673590202767048 & 0.5554647595397192 & 0.1809444152888104\end{array}$ $\begin{array}{lllll}0.8673590912880680 & 0.5554647437069410 & 0.1809444053563834\end{array}$ $\begin{array}{llllll}0.3670647051433374 & 0.0553612986546564 & 0.3277205536217201\end{array}$ $\begin{array}{lllll}0.8670646897495425 & 0.0553613439871856 & 0.3277205622712941\end{array}$ $\begin{array}{lllll}0.3682545151303092 & 0.5552325863119869 & 0.4746838419631648\end{array}$ $\begin{array}{lllll}0.8682546725918645 & 0.5552325949536080 & 0.4746838370334251\end{array}$ $\begin{array}{lllll}0.2529433811326816 & 0.1046324046590017 & 0.0031175821582323\end{array}$ $\begin{array}{lllll}0.7529433331151145 & 0.1046324288119185 & 0.0031175493680076\end{array}$ $\begin{array}{llllll}0.25388162648698855 & 0.6049219458778362 & 0.1501413560581630\end{array}$ $\begin{array}{llllll}0.7538818148775920 & 0.6049219967108824 & 0.1501413225452203\end{array}$ $\begin{array}{lllll}0.2531046397129724 & 0.1051773943169196 & 0.2971103926387594\end{array}$ $\begin{array}{lllll}0.7531046191161357 & 0.1051775128960440 & 0.2971104184672116\end{array}$ $\begin{array}{lllll}0.2543652997148639 & 0.6045181359435567 & 0.4439640282814796\end{array}$ $\begin{array}{lllll}0.7543653734818219 & 0.6045180455907448 & 0.4439640272463638\end{array}$

\section{BQ molecule on PETN (110) surface Method: PBE}

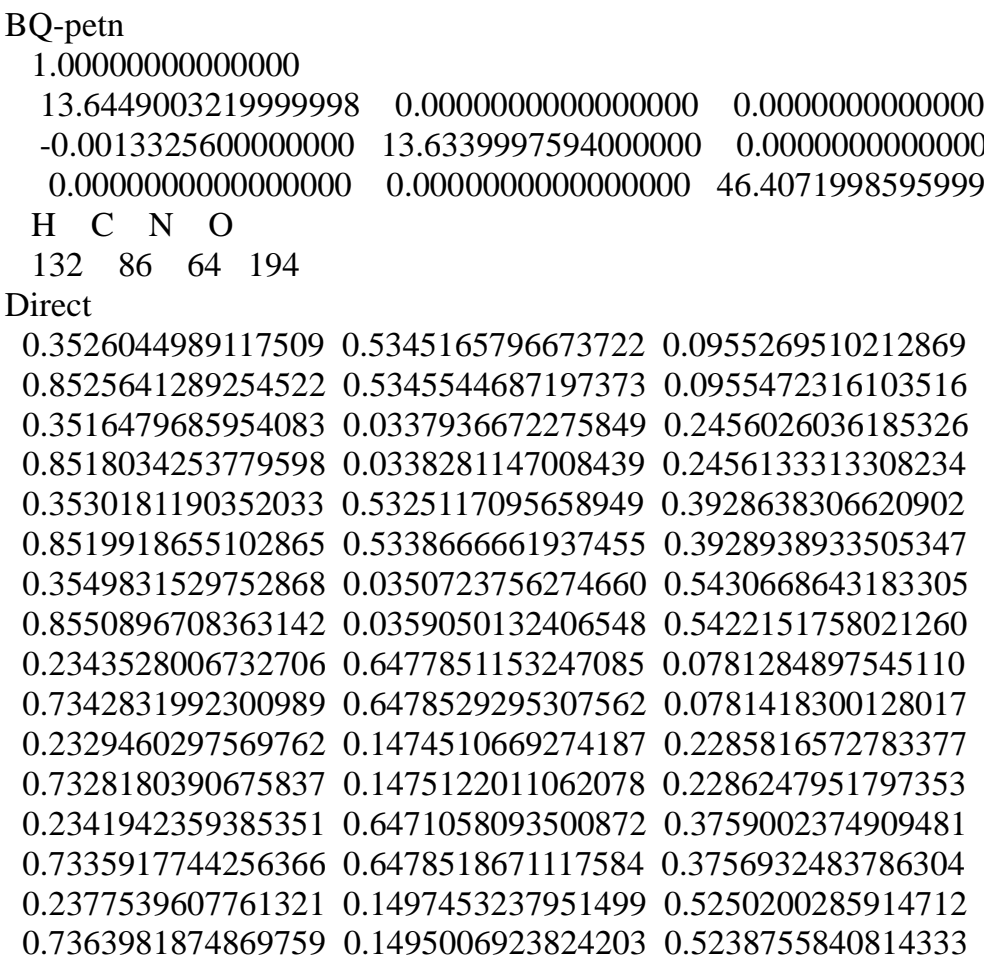


$\begin{array}{llll}0.3514655995445606 & 0.4644063980438347 & 0.0257823530970003\end{array}$ $\begin{array}{llll}0.8513472561065635 & 0.4643945662624411 & 0.0258032253761170\end{array}$ $\begin{array}{llll}0.3525115282599288 & 0.9665586185956696 & 0.1758933164203347\end{array}$ $\begin{array}{llll}0.8526081746417693 & 0.9668322962928015 & 0.1759200732557735\end{array}$ $\begin{array}{llll}0.3512630699629796 & 0.4655314551711000 & 0.3231471472487814\end{array}$ $\begin{array}{llll}0.8511698859387931 & 0.4656231522856771 & 0.3231586796951405\end{array}$ $\begin{array}{llll}0.3539961262361475 & 0.9655168458788094 & 0.4730592517724299\end{array}$ $\begin{array}{llll}0.8550577677094295 & 0.9669489474704019 & 0.4723834210948370\end{array}$ $\begin{array}{llll}0.2330389686833881 & 0.3505781987799752 & 0.0441487116539921\end{array}$ $\begin{array}{llll}0.7329872213582964 & 0.3506279980515232 & 0.0441640430142064\end{array}$ $\begin{array}{llll}0.2334414903826660 & 0.8523934359934013 & 0.1929939751873491\end{array}$ $\begin{array}{llll}0.7336978606358010 & 0.8526180762375973 & 0.1929321748087529\end{array}$ $\begin{array}{llll}0.2322782750628634 & 0.3519526389655557 & 0.3404624731728219\end{array}$ $\begin{array}{llll}0.7323648778769107 & 0.3523434359791454 & 0.3404868469559386\end{array}$ $\begin{array}{llll}0.2352256582684499 & 0.8526688340931335 & 0.4908070706702213\end{array}$ $\begin{array}{llll}0.7370689597156559 & 0.8524623009302672 & 0.4898016773902206\end{array}$ $\begin{array}{llll}0.1441137677506896 & 0.3837328337930546 & 0.0707416477905375\end{array}$ $\begin{array}{llll}0.6440640052602460 & 0.3837412124492118 & 0.0707635672279497\end{array}$ $\begin{array}{llll}0.1458108228256693 & 0.8824565026954491 & 0.2202690852251800\end{array}$ $\begin{array}{llll}0.6461992526456730 & 0.8823016706923248 & 0.2202771188793817\end{array}$ $\begin{array}{llll}0.1461614415635322 & 0.3815917316755060 & 0.3681714545795068\end{array}$ $\begin{array}{llll}0.6460983166917571 & 0.3819597772387359 & 0.3681690053110643\end{array}$ $\begin{array}{llll}0.1481014109720565 & 0.8841443206276538 & 0.5180535577964928\end{array}$ $\begin{array}{lllll}0.6502115707183066 & 0.8818200220220779 & 0.5173538602726957\end{array}$ $\begin{array}{llll}0.2660940455635707 & 0.4408220304222352 & 0.1043841217919669\end{array}$ $\begin{array}{llll}0.7660781648734279 & 0.4408315205543001 & 0.1044107444915195\end{array}$ $\begin{array}{lllll}0.2655347656791180 & 0.9390717254416074 & 0.2539133602878030\end{array}$ $\begin{array}{llll}0.7655651428339525 & 0.9392395371235789 & 0.2539307844454880\end{array}$ $\begin{array}{llll}0.2663317654755420 & 0.4386336478463240 & 0.4014628908993049\end{array}$ $\begin{array}{llll}0.7658233369194680 & 0.4394726815376566 & 0.4014636844107536\end{array}$ $\begin{array}{llll}0.2693563575434889 & 0.9397369716395481 & 0.5512943170411340\end{array}$ $\begin{array}{llll}0.7696574538685351 & 0.9406847277850205 & 0.5507097900331042\end{array}$ $\begin{array}{llll}0.1462927174606472 & 0.6174890992811389 & 0.0509812504504942\end{array}$ $\begin{array}{llll}0.6461627546991899 & 0.6174524232833782 & 0.0510224320004525\end{array}$ $\begin{array}{llll}0.1469553989792520 & 0.1187444732028463 & 0.2007553649917007\end{array}$ $\begin{array}{llll}0.6469156940293532 & 0.1187317446755969 & 0.2007746696023381\end{array}$ $\begin{array}{lllll}0.1465622977619253 & 0.6179238073812274 & 0.3485629879376309\end{array}$ $\begin{array}{lllll}0.6461909884882698 & 0.6181684611525091 & 0.3483334403790282\end{array}$ $\begin{array}{lllll}0.1472073485724613 & 0.1173770209667702 & 0.4988076309571659\end{array}$ $\begin{array}{lllll}0.6455387313735094 & 0.1151313531399647 & 0.4980098091686510\end{array}$ $\begin{array}{llll}0.2649380146787725 & 0.5586359837351761 & 0.0173555682629643\end{array}$ $\begin{array}{llll}0.7648486691883739 & 0.5586911469690155 & 0.0173750768458652\end{array}$ $\begin{array}{llll}0.2661482892025702 & 0.0608099409605923 & 0.1673150384443810\end{array}$ $\begin{array}{llll}0.7662453326860940 & 0.0610749143552769 & 0.1673607622296173\end{array}$ $\begin{array}{llll}0.2648508780749657 & 0.5599567164622092 & 0.3148246265800819\end{array}$ $\begin{array}{llll}0.7650957488415387 & 0.5602932803064766 & 0.3147531283318198\end{array}$ $\begin{array}{llll}0.2679078841562379 & 0.0601597432648730 & 0.4645960944620369\end{array}$ $\begin{array}{llll}0.7678866992715653 & 0.0605937761031446 & 0.4639589814715162\end{array}$ $\begin{array}{lllll}0.3961239290056970 & 0.8826861541015937 & 0.0509377915580202\end{array}$ $\begin{array}{llll}0.8962063348501770 & 0.8827842656580851 & 0.0509342340739505\end{array}$ $\begin{array}{llll}0.3970191504686661 & 0.3814895574632289 & 0.2007752600156790\end{array}$ $\begin{array}{lllll}0.8968639791649944 & 0.3815950275141625 & 0.2007364878488537\end{array}$ $\begin{array}{llll}0.3961877424354736 & 0.8819478017331827 & 0.3482979049326828\end{array}$ $\begin{array}{llll}0.8960524250719932 & 0.8821656936583598 & 0.3483875016329472\end{array}$ $\begin{array}{llll}0.3997908503653296 & 0.3798782184789966 & 0.4971320350602675\end{array}$ $\begin{array}{llll}0.8917238631609609 & 0.3851570186784762 & 0.4998773162770568\end{array}$ 
$\begin{array}{llll}0.5147877418249601 & 0.9415834226046790 & 0.0173651874138657\end{array}$ $\begin{array}{llll}0.0148649674970031 & 0.9416560358702933 & 0.0173562351955254\end{array}$ $\begin{array}{llll}0.5162447172872224 & 0.4391069221386589 & 0.1673691659863523\end{array}$ $\begin{array}{llll}0.0162999615325763 & 0.4391502160765358 & 0.1673480805461973\end{array}$ $\begin{array}{llll}0.5153372485232889 & 0.9396441639956582 & 0.3146745801428965\end{array}$ $\begin{array}{llll}0.0151037823998550 & 0.9398193464898648 & 0.3147644745640770\end{array}$ $\begin{array}{llll}0.5169660264818898 & 0.4401426634033063 & 0.4637826289903684\end{array}$ $\begin{array}{llll}0.0156750591488991 & 0.4370702961579455 & 0.4647000518880646\end{array}$ $\begin{array}{llll}0.3939928507161048 & 0.1165592395316646 & 0.0707414654434021\end{array}$ $\begin{array}{llll}0.8939913436564779 & 0.1165784886352854 & 0.0707124304369802\end{array}$ $\begin{array}{lllll}0.3957986370881232 & 0.6177879058486324 & 0.2202076444480983\end{array}$ $\begin{array}{llll}0.8959075131816078 & 0.6178886828455606 & 0.2202060712872863\end{array}$ $\begin{array}{llll}0.3962476056239474 & 0.1180756653894412 & 0.3680406714104464\end{array}$ $\begin{array}{llll}0.8963557543380376 & 0.1184765892710652 & 0.3681235229999231\end{array}$ $\begin{array}{llll}0.3982181507446869 & 0.6173429093467767 & 0.5174033062639194\end{array}$ $\begin{array}{llll}0.8987173874490023 & 0.6145041574247426 & 0.5185397327468257\end{array}$ $\begin{array}{llll}0.5159479286899462 & 0.0594640744322975 & 0.1043834248525314\end{array}$ $\begin{array}{lllll}0.0159341826110524 & 0.0595413872623807 & 0.1043741393555809\end{array}$ $\begin{array}{llll}0.5157105949160263 & 0.5611090301074475 & 0.2539360563541414\end{array}$ $\begin{array}{llll}0.0155960396893693 & 0.5610185291855060 & 0.2539259825514323\end{array}$ $\begin{array}{llll}0.5160240688189562 & 0.0606945572590203 & 0.4013584336978682\end{array}$ $\begin{array}{llll}0.0158421935868967 & 0.0606286702046160 & 0.4014699810307410\end{array}$ $\begin{array}{llll}0.5169723473221652 & 0.5549094725731222 & 0.5511741355516453\end{array}$ $\begin{array}{lllll}0.0220023719051308 & 0.5583598045348234 & 0.5513442130168812\end{array}$ $\begin{array}{llll}0.6023535172973199 & 0.9655313862407049 & 0.0955270068714213\end{array}$ $\begin{array}{llll}0.1023985125100147 & 0.9656750597741375 & 0.0955261713235464\end{array}$ $\begin{array}{llll}0.6018701057350981 & 0.4664559837609636 & 0.2455937092954676\end{array}$ $\begin{array}{llll}0.1017670722955625 & 0.4663582090645018 & 0.2455830280592619\end{array}$ $\begin{array}{llll}0.6021644840831590 & 0.9662536999035902 & 0.3927925891320632\end{array}$ $\begin{array}{llll}0.1022867496565815 & 0.9665561124378556 & 0.3928695922698218\end{array}$ $\begin{array}{lllll}0.6052009718109611 & 0.4640078756108190 & 0.5415069900158653\end{array}$ $\begin{array}{llll}0.1071542456642381 & 0.4632182813785966 & 0.5426599356755574\end{array}$ $\begin{array}{llll}0.4840649250251401 & 0.8522917006107240 & 0.0780944808406883\end{array}$ $\begin{array}{llll}0.9841251421401194 & 0.8523797447770649 & 0.0780966151077580\end{array}$ $\begin{array}{llll}0.4829101562086919 & 0.3527783837463241 & 0.2286226112278361\end{array}$ $\begin{array}{llll}0.9828422109824110 & 0.3527258366435160 & 0.2285581419553730\end{array}$ $\begin{array}{lllll}0.4836451187576102 & 0.8522759612203925 & 0.3756442510308748\end{array}$ $\begin{array}{llll}0.9837820431879607 & 0.8523620005510264 & 0.3756641382906233\end{array}$ $\begin{array}{llll}0.4837463391538939 & 0.3530203556011206 & 0.5257071651897551\end{array}$ $\begin{array}{llll}0.9877308157538597 & 0.3468384368641608 & 0.5235917762440828\end{array}$ $\begin{array}{llll}0.6012143071468934 & 0.0358935314851008 & 0.0258075386460820\end{array}$ $\begin{array}{llll}0.1012781391642808 & 0.0359819669690169 & 0.0257964268013783\end{array}$ $\begin{array}{lllll}0.6024988070343944 & 0.5335304264567908 & 0.1758838152480682\end{array}$ $\begin{array}{llll}0.1025172909149878 & 0.5335802481788723 & 0.1758747695964783\end{array}$ $\begin{array}{llll}0.6014478788614742 & 0.0343040656520853 & 0.3230982873648690\end{array}$ $\begin{array}{llll}0.1012731420740337 & 0.0344688019573634 & 0.3231655140135353\end{array}$ $\begin{array}{llll}0.6039860074282095 & 0.5338392593755986 & 0.4724844516676802\end{array}$ $\begin{array}{llll}0.1026210598604666 & 0.5313621968242338 & 0.4727186471606454\end{array}$ $\begin{array}{lllll}0.4830253620298910 & 0.1495137704560335 & 0.0441680439127047\end{array}$ $\begin{array}{llll}0.9830067060248262 & 0.1495818147850076 & 0.0441325646658847\end{array}$ $\begin{array}{llll}0.4835879922190672 & 0.6476702823875969 & 0.1929600169902601\end{array}$ $\begin{array}{llll}0.9836978488338934 & 0.6476875420620604 & 0.1929390063629319\end{array}$ $\begin{array}{llll}0.4825389150328904 & 0.1477097624071349 & 0.3403741649946527\end{array}$ $\begin{array}{lllll}0.9825625496109374 & 0.1477292557669511 & 0.3403965587373026\end{array}$ $\begin{array}{llll}0.4855061170277612 & 0.6476986179824858 & 0.4899925073874551\end{array}$ $\begin{array}{llll}0.9855609165739309 & 0.6458634398902037 & 0.4911960640310974\end{array}$ 
$\begin{array}{llll}0.4398471605707724 & 0.6630030730317172 & 0.6481835202351521\end{array}$ $\begin{array}{llll}0.5578726331070454 & 0.6058337068896741 & 0.6854184724120698\end{array}$ $\begin{array}{llll}0.6653687059218339 & 0.4624394271193301 & 0.6773996705973274\end{array}$ $\begin{array}{llll}0.6577878056935471 & 0.3644498800743444 & 0.6321819004391231\end{array}$ $\begin{array}{llll}0.1867767890518252 & 0.4103754094203235 & 0.0522613525086839\end{array}$ $\begin{array}{llll}0.6867262971784536 & 0.4103961638703296 & 0.0522849081106736\end{array}$ $\begin{array}{lllll}0.1870595334479578 & 0.9109810552224720 & 0.2017550722740272\end{array}$ $\begin{array}{llll}0.6872334243557356 & 0.9110222359761300 & 0.2017490877022580\end{array}$ $\begin{array}{llll}0.1864005085166819 & 0.4104107862645593 & 0.3495121139777224\end{array}$ $\begin{array}{lllll}0.6863198776601888 & 0.4107517167529581 & 0.3494962315798936\end{array}$ $\begin{array}{llll}0.1894662075952896 & 0.9119333839882530 & 0.4994601970885214\end{array}$ $\begin{array}{llll}0.6906380314931784 & 0.9107561312233013 & 0.4987238081763357\end{array}$ $\begin{array}{llll}0.1875984256507042 & 0.5892112257932821 & 0.0695291808636882\end{array}$ $\begin{array}{lllll}0.6875322906968589 & 0.5892214500490006 & 0.0695633789558083\end{array}$ $\begin{array}{llll}0.1868342292555690 & 0.0893638994843497 & 0.2194169185562235\end{array}$ $\begin{array}{lllll}0.6867652616211865 & 0.0893923639962871 & 0.2194446406038596\end{array}$ $\begin{array}{llll}0.1874522861721737 & 0.5888914724522057 & 0.3670852264932066\end{array}$ $\begin{array}{llll}0.6870374322642039 & 0.5894550541372053 & 0.3669035521970501\end{array}$ $\begin{array}{llll}0.1907460719815583 & 0.0902784217393964 & 0.5170622530906698\end{array}$ $\begin{array}{lllll}0.6897772262070294 & 0.0891932833964508 & 0.5162701401607505\end{array}$ $\begin{array}{llll}0.3125992026681803 & 0.4705881130182855 & 0.0871803443119510\end{array}$ $\begin{array}{lllll}0.8125569103376353 & 0.4706214434539156 & 0.0872004265635646\end{array}$ $\begin{array}{llll}0.3119453403311777 & 0.9702544786221694 & 0.2369153109033887\end{array}$ $\begin{array}{llll}0.8119948000333209 & 0.9703551103084955 & 0.2369273434679268\end{array}$ $\begin{array}{llll}0.3125411768708674 & 0.4692175742945572 & 0.3843173691355172\end{array}$ $\begin{array}{llll}0.8120529431240068 & 0.4702388129390409 & 0.3843496367721107\end{array}$ $\begin{array}{llll}0.3158772021495950 & 0.9711089509560383 & 0.5343327459916550\end{array}$ $\begin{array}{llll}0.8160645821914363 & 0.9716328334896112 & 0.5336543845616805\end{array}$ $\begin{array}{lllll}0.2499476608635147 & 0.4995536796658196 & 0.0608482612435837\end{array}$ $\begin{array}{llll}0.7498921886972416 & 0.4995964986570584 & 0.0608698476588121\end{array}$ $\begin{array}{llll}0.2496079213686447 & 0.0002055619779548 & 0.2106346388499722\end{array}$ $\begin{array}{llll}0.7496489516455556 & 0.0003241900736643 & 0.2106533594428258\end{array}$ $\begin{array}{llll}0.2495343408397600 & 0.4994000318307478 & 0.3581888767189161\end{array}$ $\begin{array}{llll}0.7492671399519425 & 0.4999513817904710 & 0.3581294344403223\end{array}$ $\begin{array}{llll}0.2530180702636795 & 0.0008593520658716 & 0.5080950902751281\end{array}$ $\begin{array}{llll}0.7530367455531033 & 0.0004361686685288 & 0.5073789861309862\end{array}$ $\begin{array}{llll}0.3120371638721360 & 0.5283865246670413 & 0.0343593507199611\end{array}$ $\begin{array}{llll}0.8119748862982444 & 0.5284286282962096 & 0.0343782065297932\end{array}$ $\begin{array}{llll}0.3123764160363491 & 0.0300742742908630 & 0.1844270448661906\end{array}$ $\begin{array}{llll}0.8124479142913741 & 0.0302979567562165 & 0.1844703872435201\end{array}$ $\begin{array}{llll}0.3115351772652348 & 0.5290682454256582 & 0.3318037053588431\end{array}$ $\begin{array}{llll}0.8115480697900951 & 0.5293242454899399 & 0.3317725540219157\end{array}$ $\begin{array}{lllll}0.3148144114295677 & 0.0296607871697463 & 0.4815893374002086\end{array}$ $\begin{array}{llll}0.8148119823828438 & 0.0303192758541257 & 0.4809814304050384\end{array}$ $\begin{array}{llll}0.4373524232044868 & 0.9108990322487162 & 0.0695070098037849\end{array}$ $\begin{array}{llll}0.9373941866646567 & 0.9109752693353040 & 0.0695099027385648\end{array}$ $\begin{array}{lllll}0.4368320067903470 & 0.4108541479053497 & 0.2194490265552274\end{array}$ $\begin{array}{llll}0.9367685814473388 & 0.4108666005092527 & 0.2194070702364441\end{array}$ $\begin{array}{lllll}0.4370946797353614 & 0.9106736082207498 & 0.3668545622226820\end{array}$ $\begin{array}{llll}0.9371462376764137 & 0.9107841617062463 & 0.3669265451112474\end{array}$ $\begin{array}{llll}0.4380631337743976 & 0.4103065024788158 & 0.5159498017534750\end{array}$ $\begin{array}{llll}0.9404178561581776 & 0.4084720223360253 & 0.5174414944728035\end{array}$ $\begin{array}{llll}0.4366616129870057 & 0.0898032622070242 & 0.0522760293938352\end{array}$ $\begin{array}{llll}0.9366701230601362 & 0.0898412476119965 & 0.0522480925404730\end{array}$ $\begin{array}{llll}0.4371518732546131 & 0.5891510959281323 & 0.2017280450483535\end{array}$ $\begin{array}{llll}0.9371917926401788 & 0.5892170156951847 & 0.2017139092878479\end{array}$ 
$\begin{array}{llll}0.4365028043092596 & 0.0892803271040573 & 0.3493793882387161\end{array}$ $\begin{array}{llll}0.9364790520467459 & 0.0894467284268876 & 0.3494640171653009\end{array}$ $\begin{array}{llll}0.4389784942214941 & 0.5891823360726370 & 0.4987417216323486\end{array}$ $\begin{array}{llll}0.9395266290515931 & 0.5867689768815770 & 0.4998177327439696\end{array}$ $\begin{array}{lllll}0.5623754724136448 & 0.0294997800871172 & 0.0871878684823304\end{array}$ $\begin{array}{llll}0.0623833420784588 & 0.0296095814219092 & 0.0871819902944770\end{array}$ $\begin{array}{llll}0.5620779679876339 & 0.5299670226502663 & 0.2369231317391699\end{array}$ $\begin{array}{llll}0.0620066382944165 & 0.5298959420949940 & 0.2369200482299935\end{array}$ $\begin{array}{llll}0.5622258162782683 & 0.0298772538692236 & 0.3842476526265136\end{array}$ $\begin{array}{llll}0.0621394759523986 & 0.0300694691083714 & 0.3843426762149904\end{array}$ $\begin{array}{llll}0.5637791232678269 & 0.5276855148952792 & 0.5336559558452280\end{array}$ $\begin{array}{llll}0.0674504320784309 & 0.5271067940091060 & 0.5341254873590893\end{array}$ $\begin{array}{llll}0.4997397664232621 & 0.0005753290695376 & 0.0608542173587689\end{array}$ $\begin{array}{llll}0.9997887694513475 & 0.0006616746861424 & 0.0608452237015037\end{array}$ $\begin{array}{lllll}0.4996904288434135 & 0.4999777490489634 & 0.2106638000227262\end{array}$ $\begin{array}{llll}-0.0003367285391430 & 0.4999833836719170 & 0.2106404369396847\end{array}$ $\begin{array}{lllll}0.4994196471258399 & 0.0001180888067914 & 0.3580345413967081\end{array}$ $\begin{array}{lllll}0.9993859283787250 & 0.0002725016433837 & 0.3581286079169092\end{array}$ $\begin{array}{lllll}0.5012606697715998 & 0.4993903217138012 & 0.5072599220885703\end{array}$ $\begin{array}{llll}0.0028284691106459 & 0.4974785224156654 & 0.5082086228065573\end{array}$ $\begin{array}{llll}0.5618779744995570 & 0.9718446349747684 & 0.0343717188960245\end{array}$ $\begin{array}{lllll}0.0619360897487074 & 0.9719254818446437 & 0.0343640724017016\end{array}$ $\begin{array}{llll}0.5624449730481093 & 0.4700228474239069 & 0.1844653155101448\end{array}$ $\begin{array}{lllll}0.0624699996897165 & 0.4700547700187067 & 0.1844507149698625\end{array}$ $\begin{array}{llll}0.5617307376141696 & 0.9706315955004375 & 0.3317033929400799\end{array}$ $\begin{array}{llll}0.0615985085179623 & 0.9707829479789364 & 0.3317789010621729\end{array}$ $\begin{array}{llll}0.5636389156932868 & 0.4702272970242596 & 0.4808987842573137\end{array}$ $\begin{array}{lllll}0.0632692010674915 & 0.4677232628336301 & 0.4815158943361212\end{array}$ $\begin{array}{llll}0.5404098629991090 & 0.4525853394299474 & 0.6130730434349693\end{array}$ $\begin{array}{llll}0.4788046101287579 & 0.5486501100641339 & 0.6170468446403130\end{array}$ $\begin{array}{lllll}0.4858396272235744 & 0.5984605068555611 & 0.6449534766219809\end{array}$ $\begin{array}{llll}0.5508186087754382 & 0.5667517598006703 & 0.6649948605665605\end{array}$ $\begin{array}{llll}0.6142257970047660 & 0.4821832066716962 & 0.6602562894005600\end{array}$ $\begin{array}{llll}0.6110372342672780 & 0.4281642508602717 & 0.6357567852710792\end{array}$ $\begin{array}{llll}0.0676879344638878 & 0.3629449812571816 & 0.0158733630527389\end{array}$ $\begin{array}{llll}0.5676488385403429 & 0.3629538481967698 & 0.0158933325506444\end{array}$ $\begin{array}{llll}0.0666321304949606 & 0.8644907488161991 & 0.1660387604860644\end{array}$ $\begin{array}{lllll}0.5665412989351287 & 0.8645058856208677 & 0.1661071538408116\end{array}$ $\begin{array}{llll}0.0649204350147996 & 0.3644370642458480 & 0.3140584332995591\end{array}$ $\begin{array}{llll}0.5650117718314738 & 0.3642980714328332 & 0.3140210465883400\end{array}$ $\begin{array}{llll}0.0699183753972246 & 0.8655293776073800 & 0.4633576923536645\end{array}$ $\begin{array}{llll}0.5702037927170182 & 0.8639456036084316 & 0.4629353705727672\end{array}$ $\begin{array}{llll}0.0689850597709443 & 0.6369799574625247 & 0.1057566223893615\end{array}$ $\begin{array}{llll}0.5689183830725515 & 0.6369676741351842 & 0.1057839009539665\end{array}$ $\begin{array}{llll}0.0653610885402773 & 0.1356149787730158 & 0.2548711256673344\end{array}$ $\begin{array}{llll}0.5652807681322005 & 0.1355650896643582 & 0.2548908880913104\end{array}$ $\begin{array}{llll}0.0667417352044019 & 0.6357317579835160 & 0.4026664531778509\end{array}$ $\begin{array}{llll}0.5662476079548207 & 0.6361610145301617 & 0.4025050566175551\end{array}$ $\begin{array}{llll}0.0735735871264544 & 0.1372595763434421 & 0.5540173502249519\end{array}$ $\begin{array}{llll}0.5720888500614362 & 0.1374382817556589 & 0.5529270060357362\end{array}$ $\begin{array}{lllll}0.4340577109726481 & 0.3510416529389558 & 0.1015913832511773\end{array}$ $\begin{array}{llll}0.9340238788389583 & 0.3510629828410313 & 0.1015966896785963\end{array}$ $\begin{array}{lllll}0.4335602981377503 & 0.8500711995521417 & 0.2506797175883927\end{array}$ $\begin{array}{llll}0.9335644567934103 & 0.8501532529543872 & 0.2507043309786558\end{array}$ $\begin{array}{llll}0.4340989748663511 & 0.3485742633121138 & 0.3978332744838047\end{array}$ $\begin{array}{llll}0.9343899654683628 & 0.3509742991119024 & 0.3981332733777590\end{array}$ 
$\begin{array}{llll}0.4381101694268209 & 0.8521753568063799 & 0.5487169867324514\end{array}$ $\begin{array}{llll}0.9355869363057596 & 0.8500028001942255 & 0.5481711584148075\end{array}$ $\begin{array}{llll}0.4324194695553161 & 0.6485087548842031 & 0.0193712651156086\end{array}$ $\begin{array}{llll}0.9323901808196594 & 0.6485112524212651 & 0.0193928899481601\end{array}$ $\begin{array}{llll}0.4349191247664417 & 0.1489963763167308 & 0.1706091154722969\end{array}$ $\begin{array}{llll}0.9350477207409390 & 0.1491826388353956 & 0.1706827893983286\end{array}$ $\begin{array}{lllll}0.4333213915252416 & 0.6489231914546441 & 0.3179115176469278\end{array}$ $\begin{array}{llll}0.9333040194056955 & 0.6491942382930189 & 0.3178869278092171\end{array}$ $\begin{array}{lllll}0.4359491383558156 & 0.1487739935673692 & 0.4667817870694039\end{array}$ $\begin{array}{llll}0.9358535134134733 & 0.1509628940519105 & 0.4669889855183495\end{array}$ $\begin{array}{lllll}0.6839913004588467 & 0.1488906451916802 & 0.1015621977555151\end{array}$ $\begin{array}{llll}0.1840662655503866 & 0.1489418398491725 & 0.1015812274060165\end{array}$ $\begin{array}{llll}0.6836137331075192 & 0.6503057864788195 & 0.2506457503081792\end{array}$ $\begin{array}{llll}0.1836654765236033 & 0.6499828294740706 & 0.2507132884376549\end{array}$ $\begin{array}{lllll}0.6848373595075280 & 0.1489047718020774 & 0.3980547056032191\end{array}$ $\begin{array}{lllll}0.1846466671905927 & 0.1491648325194324 & 0.3981183573648067\end{array}$ $\begin{array}{llll}0.6852361993723527 & 0.6472935171405563 & 0.5479136870927209\end{array}$ $\begin{array}{llll}0.1843340305313282 & 0.6522706069911151 & 0.5481589097078498\end{array}$ $\begin{array}{llll}0.6821142273717492 & 0.8514929235198653 & 0.0194631246470452\end{array}$ $\begin{array}{lllll}0.1821282534247856 & 0.8515077772913382 & 0.0194603260150461\end{array}$ $\begin{array}{llll}0.6849780191164733 & 0.3510850065881639 & 0.1706846230766018\end{array}$ $\begin{array}{llll}0.1850418047024942 & 0.3511285033427776 & 0.1706767161544688\end{array}$ $\begin{array}{llll}0.6835146265767644 & 0.8508806261348685 & 0.3178307909975910\end{array}$ $\begin{array}{lllll}0.1834441186797201 & 0.8510728263728587 & 0.3179098201343975\end{array}$ $\begin{array}{llll}0.6850377251480591 & 0.3501475739467112 & 0.4668988836140454\end{array}$ $\begin{array}{llll}0.1857047309968879 & 0.3494391313068841 & 0.4670793389809613\end{array}$ $\begin{array}{llll}0.3188012676037245 & 0.8630980423713109 & 0.1057411651278661\end{array}$ $\begin{array}{llll}0.8188348478102055 & 0.8631766505853661 & 0.1057445674469108\end{array}$ $\begin{array}{llll}0.3153249570453138 & 0.3645786096956328 & 0.2548762894189021\end{array}$ $\begin{array}{lllll}0.8153633032635876 & 0.3645638692889356 & 0.2548590750903783\end{array}$ $\begin{array}{llll}0.3165510865079518 & 0.8641053488363505 & 0.4025755239941606\end{array}$ $\begin{array}{llll}0.8166603479401170 & 0.8640165447366905 & 0.4025876682371098\end{array}$ $\begin{array}{llll}0.3202284880604552 & 0.3662480964280371 & 0.5527968093767557\end{array}$ $\begin{array}{lllll}0.8354712702567751 & 0.3580760960897564 & 0.5572259518122661\end{array}$ $\begin{array}{llll}0.3175854579519224 & 0.1372559736523292 & 0.0159091756111770\end{array}$ $\begin{array}{llll}0.8174651576130324 & 0.1372269013099051 & 0.0159282553176393\end{array}$ $\begin{array}{lllll}0.3170034009767738 & 0.6351715150082607 & 0.1658594987151704\end{array}$ $\begin{array}{llll}0.8169058081171562 & 0.6353840842525804 & 0.1659127937948378\end{array}$ $\begin{array}{llll}0.3150731475603432 & 0.1357138892260250 & 0.3139765497483449\end{array}$ $\begin{array}{llll}0.8149986541948392 & 0.1356968719216788 & 0.3140445269591581\end{array}$ $\begin{array}{llll}0.3185578301790971 & 0.6364150652392725 & 0.4630542800859706\end{array}$ $\begin{array}{llll}0.8214941595917118 & 0.6348270695865833 & 0.4633843223598610\end{array}$ $\begin{array}{llll}0.0894212532516646 & 0.2798339873706802 & 0.0230164849109019\end{array}$ $\begin{array}{llll}0.5894154442528523 & 0.2798458371336540 & 0.0230269820560726\end{array}$ $\begin{array}{llll}0.0882099092911805 & 0.7813084481584693 & 0.1730495328394430\end{array}$ $\begin{array}{llll}0.5883903697536512 & 0.7813325751901323 & 0.1730473378668554\end{array}$ $\begin{array}{llll}0.0874593819882416 & 0.2811282098360823 & 0.3206471944904511\end{array}$ $\begin{array}{llll}0.5876586548337027 & 0.2810878213532852 & 0.3206728531458430\end{array}$ $\begin{array}{llll}0.0919273386808974 & 0.7822847944687891 & 0.4701391985201314\end{array}$ $\begin{array}{lllll}0.5934460007296011 & 0.7807437761263679 & 0.4694265812736765\end{array}$ $\begin{array}{llll}0.1204218304776847 & 0.4431644731942502 & 0.0298620575478249\end{array}$ $\begin{array}{lllll}0.6203615164539226 & 0.4431745421006965 & 0.0298821964538444\end{array}$ $\begin{array}{lllll}0.1188227345366038 & 0.9442701944534437 & 0.1799021160821917\end{array}$ $\begin{array}{llll}0.6187387553702550 & 0.9442908930157714 & 0.1799573837725877\end{array}$ $\begin{array}{llll}0.1168798770769041 & 0.4439589051524319 & 0.3280305743152102\end{array}$ $\begin{array}{llll}0.6168076298933254 & 0.4440296286263318 & 0.3279764286020619\end{array}$ 
$\begin{array}{lll}0.1212746015366190 & 0.9453076971458625 & 0.4776170876285656\end{array}$ $\begin{array}{llll}0.6214209874306059 & 0.9438167908398457 & 0.4771184286404313\end{array}$ $\begin{array}{llll}0.0080652408468427 & 0.3927775018150494 & 0.9985722252539556\end{array}$ $\begin{array}{lllll}0.5079893808378871 & 0.3927777006307184 & 0.9985988443813826\end{array}$ $\begin{array}{lllll}0.0064491503776696 & 0.8942994254947981 & 0.1487881244320478\end{array}$ $\begin{array}{llll}0.5060612441057865 & 0.8942773688182382 & 0.1489403406691884\end{array}$ $\begin{array}{lllll}0.0037645944806746 & 0.3944872063543340 & 0.2971272498414667\end{array}$ $\begin{array}{llll}0.5039286452207690 & 0.3941483944401731 & 0.2970396284072580\end{array}$ $\begin{array}{llll}0.0099707375486592 & 0.8956210249636881 & 0.4460896070548344\end{array}$ $\begin{array}{llll}0.5090539540999833 & 0.8938256555295278 & 0.4460038395267020\end{array}$ $\begin{array}{llll}0.0919965361070204 & 0.7200352735326792 & 0.0990037483060859\end{array}$ $\begin{array}{llll}0.5918403222702563 & 0.7200249226770966 & 0.0990059097484894\end{array}$ $\begin{array}{llll}0.0884730466178899 & 0.2188484646287946 & 0.2483803660390232\end{array}$ $\begin{array}{llll}0.5884250107313657 & 0.2187958213035278 & 0.2484116143906260\end{array}$ $\begin{array}{llll}0.0885527294622340 & 0.7188104405890242 & 0.3956114308441270\end{array}$ $\begin{array}{llll}0.5883611628846249 & 0.7193249809284420 & 0.3956099707308881\end{array}$ $\begin{array}{llll}0.0941182516019941 & 0.2204487626012632 & 0.5466458824479172\end{array}$ $\begin{array}{llll}0.5925194831261371 & 0.2199926012461428 & 0.5451313790648379\end{array}$ $\begin{array}{llll}0.1193428683303984 & 0.5566841095803972 & 0.0914469071870059\end{array}$ $\begin{array}{lllll}0.6193447756265325 & 0.5566600260239027 & 0.0914939905718162\end{array}$ $\begin{array}{llll}0.1169886509669409 & 0.0558470926844523 & 0.2407797578719732\end{array}$ $\begin{array}{llll}0.6169015224679425 & 0.0557946611093601 & 0.2407892536340890\end{array}$ $\begin{array}{llll}0.1189001673858013 & 0.5557807518920224 & 0.3888496631060755\end{array}$ $\begin{array}{lllll}0.6184687874273387 & 0.5563345439332638 & 0.3886599836594090\end{array}$ $\begin{array}{lllll}0.1256385163792117 & 0.0572650153422817 & 0.5397487856833001\end{array}$ $\begin{array}{llll}0.6257070738681210 & 0.0564445979997165 & 0.5391707347425768\end{array}$ $\begin{array}{lllll}0.0087265559454559 & 0.6074353532964367 & 0.1229916030306531\end{array}$ $\begin{array}{llll}0.5087136397242547 & 0.6074335100543402 & 0.1230337640218550\end{array}$ $\begin{array}{llll}0.0039841052782281 & 0.1056241562193501 & 0.2717218646425730\end{array}$ $\begin{array}{lllll}0.5038839237120371 & 0.1055840364315005 & 0.2717339320422819\end{array}$ $\begin{array}{llll}0.0063744875609740 & 0.6060779346500449 & 0.4198645756292744\end{array}$ $\begin{array}{llll}0.5055860995871430 & 0.6063051882512740 & 0.4195828130068803\end{array}$ $\begin{array}{llll}0.0155545983126064 & 0.1071590272501105 & 0.5717569324694424\end{array}$ $\begin{array}{lllll}0.5137560042606221 & 0.1076770924994817 & 0.5705958382933108\end{array}$ $\begin{array}{llll}0.4112121825141929 & 0.3752593089538402 & 0.1258775607567039\end{array}$ $\begin{array}{llll}0.9112008111767919 & 0.3752631461791482 & 0.1258861484602851\end{array}$ $\begin{array}{llll}0.4100085603813144 & 0.8721130879109953 & 0.2750923581076418\end{array}$ $\begin{array}{llll}0.9100482550482999 & 0.8722639862121353 & 0.2751140144370637\end{array}$ $\begin{array}{llll}0.4121150798317937 & 0.3715106338849710 & 0.4222888014613302\end{array}$ $\begin{array}{llll}0.9123300485190381 & 0.3747268923461580 & 0.4225197058648318\end{array}$ $\begin{array}{llll}0.4144848278827333 & 0.8753406043508978 & 0.5729798923683737\end{array}$ $\begin{array}{llll}0.9105637942995012 & 0.8714814322018961 & 0.5724571097271857\end{array}$ $\begin{array}{lllll}0.3821223089038460 & 0.3969956097518252 & 0.0777929608071646\end{array}$ $\begin{array}{llll}0.8820798076245101 & 0.3970446464261421 & 0.0778088574158932\end{array}$ $\begin{array}{llll}0.3819291706372534 & 0.8975909107582353 & 0.2271598471483421\end{array}$ $\begin{array}{llll}0.8819146391860235 & 0.8975950931919456 & 0.2271789376004364\end{array}$ $\begin{array}{llll}0.3815162021243475 & 0.3958018757665933 & 0.3744219098043212\end{array}$ $\begin{array}{llll}0.8815910838229324 & 0.3972230109764107 & 0.3745949623383377\end{array}$ $\begin{array}{lllll}0.3862456950590674 & 0.8987846670668845 & 0.5248059836497602\end{array}$ $\begin{array}{llll}0.8865521822508261 & 0.8991323845737154 & 0.5242788886576727\end{array}$ $\begin{array}{llll}0.4953408561840187 & 0.2929611494370556 & 0.0931412127075181\end{array}$ $\begin{array}{lllll}0.9953007117723985 & 0.2929776675119047 & 0.0931442395688538\end{array}$ $\begin{array}{lllll}0.4953659813830006 & 0.7930320642701834 & 0.2419430260900541\end{array}$ $\begin{array}{llll}0.9953709788597668 & 0.7931072581249455 & 0.2419751827510456\end{array}$ $\begin{array}{llll}0.4949774843908404 & 0.2907926728493541 & 0.3889307941190363\end{array}$ $\begin{array}{llll}0.9955068035673097 & 0.2931646005942919 & 0.3894042067500479\end{array}$ 
$\begin{array}{llll}0.5000614995451499 & 0.7949538290245347 & 0.5401378412519856\end{array}$ $\begin{array}{llll}0.9971707741748544 & 0.7922861415163558 & 0.5396051539896094\end{array}$ $\begin{array}{llll}0.4078827096919034 & 0.6252064915387822 & 0.9952030552235580\end{array}$ $\begin{array}{llll}0.9078395482667977 & 0.6252221225185913 & 0.9952252566400738\end{array}$ $\begin{array}{llll}0.4129164951216910 & 0.1250679770878093 & 0.1462130539803939\end{array}$ $\begin{array}{llll}0.9130765566106811 & 0.1252709755464080 & 0.1462844988014086\end{array}$ $\begin{array}{llll}0.4101970126362599 & 0.6262227938080285 & 0.2934959993407545\end{array}$ $\begin{array}{llll}0.9099841309453566 & 0.6266025268630973 & 0.2934794793735009\end{array}$ $\begin{array}{llll}0.4115078706363652 & 0.1250194272352141 & 0.4425712055205715\end{array}$ $\begin{array}{llll}0.9129548444523485 & 0.1278822048404090 & 0.4425967723688735\end{array}$ $\begin{array}{llll}0.3821550698631335 & 0.6016274956282232 & 0.0434847979536540\end{array}$ $\begin{array}{llll}0.8820988762876504 & 0.6016396393278958 & 0.0435063607982675\end{array}$ $\begin{array}{llll}0.3819261443529299 & 0.1032825817300708 & 0.1941306681458811\end{array}$ $\begin{array}{llll}0.8819675216675837 & 0.1034954123820696 & 0.1942024721134646\end{array}$ $\begin{array}{lllll}0.3814838955426326 & 0.6020387819153197 & 0.3414279952260350\end{array}$ $\begin{array}{llll}0.8816535231242770 & 0.6021048534629861 & 0.3414137487120646\end{array}$ $\begin{array}{llll}0.3853611649837225 & 0.1026097280473718 & 0.4907610451310423\end{array}$ $\begin{array}{llll}0.8842136451932884 & 0.1039047058786453 & 0.4905427210891561\end{array}$ $\begin{array}{llll}0.4942413706481216 & 0.7064670307100822 & 0.0276762542316141\end{array}$ $\begin{array}{llll}0.9942447179773567 & 0.7064417552712284 & 0.0276959960344528\end{array}$ $\begin{array}{llll}0.4961877000545053 & 0.2068074811571285 & 0.1792620368204926\end{array}$ $\begin{array}{llll}0.9963245684785436 & 0.2069516417055768 & 0.1793443412924268\end{array}$ $\begin{array}{llll}0.4949571341569082 & 0.7062882011421399 & 0.3265758806853795\end{array}$ $\begin{array}{llll}0.9949740150764521 & 0.7065570073796785 & 0.3265314847643106\end{array}$ $\begin{array}{llll}0.4978929611735119 & 0.2065354834621770 & 0.4749743110728009\end{array}$ $\begin{array}{llll}0.9970724666436959 & 0.2088074856275495 & 0.4756452019381903\end{array}$ $\begin{array}{llll}0.6612480744163081 & 0.1245843040702664 & 0.1258494169421678\end{array}$ $\begin{array}{llll}0.1612860050066279 & 0.1246290436198328 & 0.1258638281645253\end{array}$ $\begin{array}{llll}0.6602443936283350 & 0.6281283580930599 & 0.2750647925970831\end{array}$ $\begin{array}{llll}0.1602167249527551 & 0.6277074549634465 & 0.2751169227474841\end{array}$ $\begin{array}{llll}0.6629243123960433 & 0.1249574826705840 & 0.4224365390955008\end{array}$ $\begin{array}{llll}0.1629645691703221 & 0.1250429083731725 & 0.4225025898923447\end{array}$ $\begin{array}{llll}0.6665327709046591 & 0.6197051949606062 & 0.5722097545839886\end{array}$ $\begin{array}{llll}0.1576261702089183 & 0.6335021619093151 & 0.5724480036799641\end{array}$ $\begin{array}{llll}0.6319510321673164 & 0.1029991284327960 & 0.0777756195753644\end{array}$ $\begin{array}{llll}0.1319585390566736 & 0.1031542965874289 & 0.0777867283441097\end{array}$ $\begin{array}{llll}0.6319702269728465 & 0.6026912637392010 & 0.2271409426069635\end{array}$ $\begin{array}{llll}0.1319447139863575 & 0.6026329751597499 & 0.2271790415607491\end{array}$ $\begin{array}{llll}0.6318624436022074 & 0.1028829744243179 & 0.3745087919506143\end{array}$ $\begin{array}{llll}0.1314452009083742 & 0.1033293951536945 & 0.3745945598762084\end{array}$ $\begin{array}{lllll}0.6317387536997857 & 0.6033347327161265 & 0.5243841952658984\end{array}$ $\begin{array}{lllll}0.1373451980782956 & 0.5995181988434137 & 0.5243674034321759\end{array}$ $\begin{array}{lllll}0.7452609828586847 & 0.2069887872421361 & 0.0931068257776637\end{array}$ $\begin{array}{llll}0.2454043126802081 & 0.2069968900870315 & 0.0931386913690934\end{array}$ $\begin{array}{llll}0.7452011564782030 & 0.7075519259198638 & 0.2418822789584616\end{array}$ $\begin{array}{llll}0.2454199782800953 & 0.7071292760509611 & 0.2419990311086625\end{array}$ $\begin{array}{llll}0.7459423340243927 & 0.2067791224891810 & 0.3893475893805269\end{array}$ $\begin{array}{llll}0.2456303679688520 & 0.2071093787984753 & 0.3893809276249961\end{array}$ $\begin{array}{llll}0.7443121100677046 & 0.7080284305645909 & 0.5394898322591443\end{array}$ $\begin{array}{llll}0.2456307683992866 & 0.7097402807170011 & 0.5393303891869369\end{array}$ $\begin{array}{llll}0.3419500077190676 & 0.7800579095889925 & 0.0990248647553827\end{array}$ $\begin{array}{llll}0.8419814598316008 & 0.7801416379224835 & 0.0990177153258961\end{array}$ $\begin{array}{llll}0.3384521038549385 & 0.2813633364572624 & 0.2483758422243795\end{array}$ $\begin{array}{lllll}0.8385020581145057 & 0.2813620617861309 & 0.2483457338391969\end{array}$ $\begin{array}{llll}0.3383673085569334 & 0.7809185107783387 & 0.3956461672882066\end{array}$ $\begin{array}{lllll}0.8383924728437437 & 0.7808979002737715 & 0.3955613729933288\end{array}$ 
$\begin{array}{llll}0.3465097332954480 & 0.2825947604288825 & 0.5477229155589467\end{array}$ $\begin{array}{llll}0.8491872306476430 & 0.2759133985963539 & 0.5477667434554190\end{array}$ $\begin{array}{llll}0.6577357844872876 & 0.8747379365335008 & 0.9952750995137202\end{array}$ $\begin{array}{llll}0.1577658727753573 & 0.8747165450021599 & 0.9952681725627498\end{array}$ $\begin{array}{llll}0.6629695497233721 & 0.3749566929855363 & 0.1462871978029202\end{array}$ $\begin{array}{llll}0.1630225244457908 & 0.3749284599067118 & 0.1462729328835926\end{array}$ $\begin{array}{lllll}0.6601200756643343 & 0.8733351050456316 & 0.2934186983002760\end{array}$ $\begin{array}{lllll}0.1603315024130406 & 0.8738697695392206 & 0.2935001870847491\end{array}$ $\begin{array}{llll}0.6628215026819849 & 0.3739173246041976 & 0.4424923696482820\end{array}$ $\begin{array}{llll}0.1632288176767547 & 0.3742738038046186 & 0.4427950807181077\end{array}$ $\begin{array}{llll}0.6319416235190422 & 0.8986772364745456 & 0.0435303195598119\end{array}$ $\begin{array}{llll}0.1319970579667809 & 0.8987683584552790 & 0.0435312214475506\end{array}$ $\begin{array}{llll}0.6320569089605095 & 0.3969606271725153 & 0.1942044643289089\end{array}$ $\begin{array}{llll}0.1321269628447675 & 0.3970287860320011 & 0.1941898778147246\end{array}$ $\begin{array}{llll}0.6317812242845743 & 0.8978397798588125 & 0.3413588264626732\end{array}$ $\begin{array}{lllll}0.1316230565964805 & 0.8979518302520914 & 0.3414291577431007\end{array}$ $\begin{array}{lllll}0.6329556388541332 & 0.3965065496158431 & 0.4904196265368338\end{array}$ $\begin{array}{lllll}0.1334577618275377 & 0.3945594225362467 & 0.4908012359052930\end{array}$ $\begin{array}{lllll}0.7436755652393939 & 0.7933967104883984 & 0.0278388698499781\end{array}$ $\begin{array}{llll}0.2436708172871511 & 0.7934148560146675 & 0.0278514090239036\end{array}$ $\begin{array}{llll}0.7461976241620999 & 0.2932645971792222 & 0.1793506388987920\end{array}$ $\begin{array}{llll}0.2462724056744200 & 0.2933445677432202 & 0.1793542980930171\end{array}$ $\begin{array}{llll}0.7453208587497430 & 0.7937083015657310 & 0.3264875650593937\end{array}$ $\begin{array}{llll}0.2450262580866694 & 0.7936625412191349 & 0.3265673138469394\end{array}$ $\begin{array}{lllll}0.7460382430561826 & 0.2919618127999757 & 0.4755035066657466\end{array}$ $\begin{array}{llll}0.2469231006408159 & 0.2911165876148570 & 0.4754507992362929\end{array}$ $\begin{array}{llll}0.3691196361378115 & 0.9434037284390686 & 0.0914256146517342\end{array}$ $\begin{array}{llll}0.8691549632750822 & 0.9434748192834971 & 0.0914225745683181\end{array}$ $\begin{array}{llll}0.3669309856588055 & 0.4443895439555285 & 0.2407878508815017\end{array}$ $\begin{array}{lllll}0.8669315082868017 & 0.4443853539865263 & 0.2407715588526892\end{array}$ $\begin{array}{lllll}0.3686247030825206 & 0.9439078447823200 & 0.3886167274265672\end{array}$ $\begin{array}{llll}0.8688400056554404 & 0.9438743717639546 & 0.3887560059759855\end{array}$ $\begin{array}{lllll}0.3665235588528039 & 0.4446585923539241 & 0.5366062214269737\end{array}$ $\begin{array}{llll}0.8822778650220682 & 0.4394292352739062 & 0.5419751417423375\end{array}$ $\begin{array}{llll}0.2584750809892701 & 0.8926389657945896 & 0.1229506518708313\end{array}$ $\begin{array}{llll}0.7585137280947902 & 0.8927170544713727 & 0.1229568814501900\end{array}$ $\begin{array}{llll}0.2539820676670967 & 0.3945298296091282 & 0.2717394278381887\end{array}$ $\begin{array}{llll}0.7540239266296288 & 0.3944709426180045 & 0.2717306261033710\end{array}$ $\begin{array}{llll}0.2562842567115088 & 0.8940660563976311 & 0.4197545206481602\end{array}$ $\begin{array}{llll}0.7563556843274132 & 0.8937773378719650 & 0.4197943173649554\end{array}$ $\begin{array}{llll}0.2590552171084545 & 0.3971176534276359 & 0.5695012038708998\end{array}$ $\begin{array}{llll}0.7882071046081365 & 0.3861495322393934 & 0.5777689932092011\end{array}$ $\begin{array}{lllll}0.3392382751045687 & 0.2203283135902769 & 0.0231159476118423\end{array}$ $\begin{array}{llll}0.8389895960287472 & 0.2202947149511608 & 0.0231762268590883\end{array}$ $\begin{array}{llll}0.3382040139403448 & 0.7184464008726360 & 0.1728634967820199\end{array}$ $\begin{array}{lllll}0.8382244377429341 & 0.7186259872267434 & 0.1729215961582113\end{array}$ $\begin{array}{lllll}0.3375525976231134 & 0.2189117015984262 & 0.3207057639284989\end{array}$ $\begin{array}{llll}0.8375953181777780 & 0.2189519087853606 & 0.3206743122447797\end{array}$ $\begin{array}{llll}0.3409694813603397 & 0.7196306908020913 & 0.4698523440277617\end{array}$ $\begin{array}{llll}0.8455145076447450 & 0.7178437033210758 & 0.4698397167410579\end{array}$ $\begin{array}{llll}0.3703467375772420 & 0.0570453136705184 & 0.0298534225111630\end{array}$ $\begin{array}{lllll}0.8703862478877924 & 0.0570362685680175 & 0.0298254408282952\end{array}$ $\begin{array}{llll}0.3690577716705222 & 0.5556471453028701 & 0.1798558965669527\end{array}$ $\begin{array}{llll}0.8690363990862128 & 0.5557744203323106 & 0.1798576117009664\end{array}$ $\begin{array}{llll}0.3670077189582979 & 0.0559574123295936 & 0.3278573663599881\end{array}$ $\begin{array}{llll}0.8668834080546159 & 0.0560469933030968 & 0.3279935828795000\end{array}$ 
$\begin{array}{llll}0.3700885878092788 & 0.5565938934634527 & 0.4769337318235432\end{array}$ $\begin{array}{llllll}0.8706568089231180 & 0.5544197546123308 & 0.4780250092034459\end{array}$ $\begin{array}{lllll}0.2580391768618100 & 0.1075035724581205 & 0.9985708527833717\end{array}$ $\begin{array}{lllll}0.7579351239592755 & 0.1074702464696649 & 0.9985862928101358\end{array}$ $\begin{array}{lllll}0.2572799184937912 & 0.6050900566634002 & 0.1485092192586228\end{array}$ $\begin{array}{lllll}0.7570001621802255 & 0.6053819054926828 & 0.1486048130020923\end{array}$ $\begin{array}{lllll}0.2540325549572519 & 0.1059308390645632 & 0.2969690064566470\end{array}$ $\begin{array}{lllll}0.7538725553196302 & 0.1057625516043174 & 0.2970870150438143\end{array}$ $\begin{array}{llllll}0.2578122827320841 & 0.6068410763476803 & 0.4459223072693935\end{array}$ $\begin{array}{lllll}0.7610521562810354 & 0.6053544546175670 & 0.4461847718696413\end{array}$ $\begin{array}{lllll}0.5268246280298097 & 0.4008539405156782 & 0.5917328584709909\end{array}$ $\begin{array}{llll}0.4290057676603927 & 0.5798504240179359 & 0.5969600147860979\end{array}$

\section{BQ molecule in triplet $\mathrm{T1}^{3}\left(\mathrm{n}, \pi^{*}\right)$ state on PETN (110) surface Method: PBE}

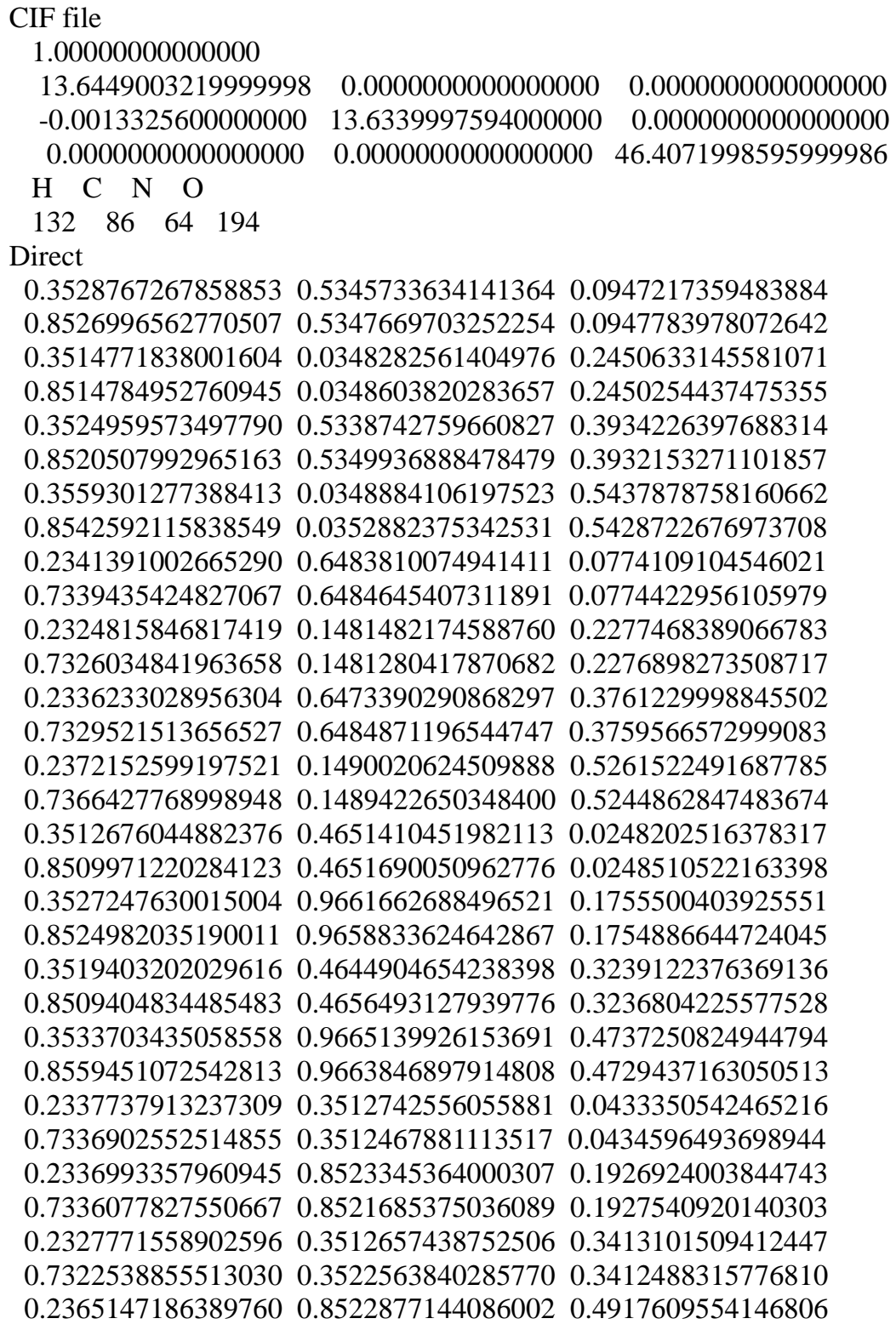


$\begin{array}{llll}0.7371058154337090 & 0.8523253624867605 & 0.4900561096033885\end{array}$ $\begin{array}{lllll}0.1444682335132281 & 0.3843406995905118 & 0.0698040935893761\end{array}$ $\begin{array}{lllll}0.6444681243417342 & 0.3843976439983278 & 0.0699472862545302\end{array}$ $\begin{array}{lllll}0.1458627749843983 & 0.8824726950772407 & 0.2199116728450992\end{array}$ $\begin{array}{lllll}0.6457364523383831 & 0.8825897140243268 & 0.2199403366385701\end{array}$ $\begin{array}{llllll}0.1461049941717917 & 0.3816718022580142 & 0.3688130898309454\end{array}$ $\begin{array}{llllll}0.6454452508399190 & 0.3827838412953756 & 0.3687063240798988\end{array}$ $\begin{array}{lllll}0.1486568575194974 & 0.8831222210933625 & 0.5188978339063482\end{array}$ $\begin{array}{lllll}0.6497905542732649 & 0.8819512652343201 & 0.5174578812895784\end{array}$ $\begin{array}{lllll}0.2662972846721163 & 0.4407061271936719 & 0.1034265463767147\end{array}$ $\begin{array}{lllll}0.7663886379435849 & 0.4406869696638743 & 0.1034695059176438\end{array}$ $\begin{array}{lllll}0.2652499455270160 & 0.9404040835801075 & 0.2535790250144431\end{array}$ $\begin{array}{lllll}0.7653043403380333 & 0.9403793003546601 & 0.2535336688736469\end{array}$ $\begin{array}{lllll}0.2662403145257090 & 0.4398489710511353 & 0.4022042873064079\end{array}$ $\begin{array}{lllll}0.7657950500557585 & 0.4409181991961447 & 0.4020326546476486\end{array}$ $\begin{array}{lllll}0.2706136842038629 & 0.9395527389752739 & 0.5522638698242213\end{array}$ $\begin{array}{lllll}0.7685766710085277 & 0.9400939252286137 & 0.5511111533906420\end{array}$ $\begin{array}{lllll}0.1460049112749280 & 0.6178158849072767 & 0.0502902156086504\end{array}$ $\begin{array}{lllll}0.6458130365290284 & 0.6178184965907625 & 0.0503306750606515\end{array}$ $\begin{array}{llllll}0.1461580820833743 & 0.1182697082027596 & 0.2001003445294640\end{array}$ $\begin{array}{lllll}0.6461789407989107 & 0.1182463616299790 & 0.2000700745671269\end{array}$ $\begin{array}{lllll}0.1460316251885388 & 0.6170030668415502 & 0.3488553234114889\end{array}$ $\begin{array}{llllll}0.6452419560182834 & 0.6180771137333628 & 0.3487270420774032\end{array}$ $\begin{array}{lllll}0.1466618838370246 & 0.1166300045652130 & 0.4999433548202728\end{array}$ $\begin{array}{lllll}0.6468845305952862 & 0.1157810867267080 & 0.4981633222689638\end{array}$ $\begin{array}{lllll}0.2655188542233551 & 0.5603700062636263 & 0.0166065895110402\end{array}$ $\begin{array}{lllll}0.7652195482059302 & 0.5603311367243203 & 0.0166531687444777\end{array}$ $\begin{array}{lllll}0.2661921669056993 & 0.0599390524250749 & 0.1666985098207793\end{array}$ $\begin{array}{lllll}0.7660109533591400 & 0.0596531203256509 & 0.1666283915322951\end{array}$ $\begin{array}{lllll}0.2653032013805209 & 0.5583710029737139 & 0.3152087755672445\end{array}$ $\begin{array}{lllll}0.7645901663839030 & 0.5599177525792643 & 0.3150903780935464\end{array}$ $\begin{array}{lllll}0.2670792876623944 & 0.0612617915012126 & 0.4656814207216924\end{array}$ $\begin{array}{lllll}0.7691645494546426 & 0.0602110710028659 & 0.4643893867357554\end{array}$ $\begin{array}{lllll}0.3959632001177253 & 0.8822890440888509 & 0.0503706588982953\end{array}$ $\begin{array}{lllll}0.8960269902268259 & 0.8820827554508794 & 0.0504140484651368\end{array}$ $\begin{array}{lllll}0.3962529502406045 & 0.3815702317272907 & 0.2003637685242294\end{array}$ $\begin{array}{llllll}0.8961931192786612 & 0.3815083180473047 & 0.2002447790193886\end{array}$ $\begin{array}{lllll}0.3951933832880383 & 0.8816135624566943 & 0.3487647392358756\end{array}$ $\begin{array}{lllll}0.8961430094995602 & 0.8824556046708737 & 0.3487601450432385\end{array}$ $\begin{array}{lllll}0.4000314066802084 & 0.3806941106900633 & 0.4977448889917583\end{array}$ $\begin{array}{lllll}0.8931341836082103 & 0.3845977736518601 & 0.5009797513733529\end{array}$ $\begin{array}{lllll}0.5153688681403445 & 0.9398888128903197 & 0.0166624926428319\end{array}$ $\begin{array}{lllll}0.0152283170222476 & 0.9397820588493172 & 0.0167113711673877\end{array}$ $\begin{array}{lllll}0.5157666305243545 & 0.4401298289819703 & 0.1668915760125892\end{array}$ $\begin{array}{lllll}0.0159061030908417 & 0.4400514489408037 & 0.1668016782575253\end{array}$ $\begin{array}{lllll}0.5142971459618340 & 0.9398218739846710 & 0.3150607043114274\end{array}$ $\begin{array}{lllll}0.0154103131512651 & 0.9410786087196882 & 0.3151515463765100\end{array}$ $\begin{array}{lllll}0.5181178248458916 & 0.4402818578048994 & 0.4640777017654109\end{array}$ $\begin{array}{lllll}0.0160000249695627 & 0.4376978547350863 & 0.4661875913013974\end{array}$ $\begin{array}{llllll}0.3942605887674139 & 0.1156692248263934 & 0.0698682419750359\end{array}$ $\begin{array}{lllll}0.8943262241026008 & 0.1155745036927339 & 0.0699383540997764\end{array}$ $\begin{array}{lllll}0.3953865964576923 & 0.6172818272554635 & 0.2200956535138505\end{array}$ $\begin{array}{lllll}0.8954631332534013 & 0.6172373460530465 & 0.2200138974320403\end{array}$ $\begin{array}{llllll}0.3951881090090371 & 0.1172217021496394 & 0.3686368531360238\end{array}$ $\begin{array}{lllll}0.8962601935858481 & 0.1176760521379095 & 0.3688421519677598\end{array}$ $\begin{array}{llll}0.3973487170613577 & 0.6168224428902715 & 0.5174546721445648\end{array}$ 
$\begin{array}{llll}0.8994654508283707 & 0.6151037412924218 & 0.5199942810404311\end{array}$ $\begin{array}{llll}0.5163470796774239 & 0.0594230079493677 & 0.1034769559555018\end{array}$ $\begin{array}{llll}0.0163779953726724 & 0.0593250551867070 & 0.1035076445609440\end{array}$ $\begin{array}{llll}0.5152789373701403 & 0.5600717202746408 & 0.2537225616695802\end{array}$ $\begin{array}{llll}0.0151974591371565 & 0.5598384684635699 & 0.2536493127216702\end{array}$ $\begin{array}{llll}0.5157591862863186 & 0.0594122920175703 & 0.4019515405672874\end{array}$ $\begin{array}{llll}0.0163659306557541 & 0.0593149576571200 & 0.4021797216235143\end{array}$ $\begin{array}{llll}0.5175614474826286 & 0.5595098838483346 & 0.5509508232163437\end{array}$ $\begin{array}{llll}0.0219823695933479 & 0.5571704915730793 & 0.5529895167208086\end{array}$ $\begin{array}{lllll}0.6029414192957687 & 0.9656374467788311 & 0.0947729929856184\end{array}$ $\begin{array}{lllll}0.1030449750343474 & 0.9656297945541649 & 0.0947942356377467\end{array}$ $\begin{array}{llll}0.6014855803933677 & 0.4656036417689841 & 0.2452509423128713\end{array}$ $\begin{array}{llll}0.1013676955710192 & 0.4653391236666883 & 0.2451679757799073\end{array}$ $\begin{array}{llll}0.6020018078582422 & 0.9653636421501218 & 0.3931179468449043\end{array}$ $\begin{array}{llll}0.1027959403695185 & 0.9655427461882119 & 0.3933191424949181\end{array}$ $\begin{array}{llll}0.6050134180998051 & 0.4667083972491370 & 0.5421320696731354\end{array}$ $\begin{array}{llll}0.1071717436431078 & 0.4622261986059429 & 0.5441255927217400\end{array}$ $\begin{array}{llll}0.4841272880218166 & 0.8518050832163292 & 0.0774917303547391\end{array}$ $\begin{array}{llll}0.9841418717450242 & 0.8516513663720811 & 0.0775512381608401\end{array}$ $\begin{array}{llll}0.4826443184737880 & 0.3520994494619636 & 0.2280140908692571\end{array}$ $\begin{array}{llll}0.9825719637056174 & 0.3519595456145088 & 0.2278958148842807\end{array}$ $\begin{array}{llll}0.4826617956702880 & 0.8517490976273674 & 0.3760969528471410\end{array}$ $\begin{array}{llll}0.9840461364281341 & 0.8518775831934070 & 0.3759174074990719\end{array}$ $\begin{array}{llll}0.4865020330527297 & 0.3529458728605748 & 0.5255360939272170\end{array}$ $\begin{array}{llll}0.9877318421942988 & 0.3473183536939309 & 0.5253108300138495\end{array}$ $\begin{array}{lllll}0.6012017113436605 & 0.0349975875481346 & 0.0249011330670281\end{array}$ $\begin{array}{lllll}0.1011515459820308 & 0.0348878874079816 & 0.0249220076493213\end{array}$ $\begin{array}{lllll}0.6023550807549889 & 0.5339448715440617 & 0.1756701465206011\end{array}$ $\begin{array}{llll}0.1024111773109745 & 0.5338970675477467 & 0.1755971899411317\end{array}$ $\begin{array}{llll}0.6005371709598123 & 0.0342646908102338 & 0.3236063284861827\end{array}$ $\begin{array}{llll}0.1020010604338821 & 0.0350206796018527 & 0.3238440894793368\end{array}$ $\begin{array}{llll}0.6042492005300996 & 0.5347290577334720 & 0.4726471179609011\end{array}$ $\begin{array}{llll}0.1027845238007996 & 0.5320370837853260 & 0.4742587687078542\end{array}$ $\begin{array}{llll}0.4836015718779320 & 0.1488983984603305 & 0.0434167116258044\end{array}$ $\begin{array}{llll}0.9836286272374670 & 0.1487654500355432 & 0.0434743402367982\end{array}$ $\begin{array}{lllll}0.4831851739449662 & 0.6478168900643057 & 0.1929047693550702\end{array}$ $\begin{array}{llll}0.9833148961429338 & 0.6477449616941408 & 0.1928324717331690\end{array}$ $\begin{array}{llll}0.4819670361490079 & 0.1476874560611295 & 0.3411616840466434\end{array}$ $\begin{array}{llll}0.9828462785684018 & 0.1482642794913201 & 0.3413465076236546\end{array}$ $\begin{array}{lllll}0.4842709118254858 & 0.6481026436871109 & 0.4900813641848168\end{array}$ $\begin{array}{llll}0.9865015990354096 & 0.6460977406966304 & 0.4926760883815659\end{array}$ $\begin{array}{llll}0.4434857617850433 & 0.6667175315015245 & 0.6480046205122999\end{array}$ $\begin{array}{llll}0.5598348757188867 & 0.6109302268858333 & 0.6859129618070340\end{array}$ $\begin{array}{llll}0.6621723315623351 & 0.4614209466439045 & 0.6783351240337233\end{array}$ $\begin{array}{llll}0.6474709932722869 & 0.3648229800197321 & 0.6330317790660864\end{array}$ $\begin{array}{lllll}0.1872033245134709 & 0.4109725702027667 & 0.0513325447463845\end{array}$ $\begin{array}{llll}0.6871432669980303 & 0.4109599733442423 & 0.0514535116979789\end{array}$ $\begin{array}{llll}0.1871521633821583 & 0.9109093664657925 & 0.2013869640908272\end{array}$ $\begin{array}{llll}0.6870543249615390 & 0.9108234014520430 & 0.2013971202680449\end{array}$ $\begin{array}{llll}0.1867567822249817 & 0.4099664959420068 & 0.3501561292827354\end{array}$ $\begin{array}{llll}0.6861233655847319 & 0.4109687352150833 & 0.3500373726669228\end{array}$ $\begin{array}{llll}0.1902038898331614 & 0.9111986447203646 & 0.5003767942870511\end{array}$ $\begin{array}{llll}0.6907218831929731 & 0.9107446740492641 & 0.4989168081791540\end{array}$ $\begin{array}{llll}0.1876609899723785 & 0.5895918204563968 & 0.0687824195411415\end{array}$ $\begin{array}{llll}0.6874707248638035 & 0.5896604630273118 & 0.0688305404255288\end{array}$ $\begin{array}{llll}0.1864914528136220 & 0.0896231000909936 & 0.2187747187838745\end{array}$ 
$\begin{array}{llll}0.6865688960656190 & 0.0895951971245766 & 0.2187376868369423\end{array}$ $\begin{array}{llll}0.1871827271875510 & 0.5886981484360418 & 0.3674219904034116\end{array}$ $\begin{array}{llll}0.6864851689096346 & 0.5898135349511305 & 0.3672829204976023\end{array}$ $\begin{array}{llll}0.1903361350556300 & 0.0894787090139928 & 0.5181602939485447\end{array}$ $\begin{array}{llll}0.6901064819879297 & 0.0890999840133879 & 0.5165345453751110\end{array}$ $\begin{array}{llll}0.3128136624812987 & 0.4708285865883817 & 0.0862712125172025\end{array}$ $\begin{array}{llll}0.8127749833408469 & 0.4709904234429243 & 0.0863139431635937\end{array}$ $\begin{array}{llll}0.3117330903029251 & 0.9710868659015504 & 0.2365128310818298\end{array}$ $\begin{array}{llll}0.8117798788499238 & 0.9710825905152645 & 0.2364748484263334\end{array}$ $\begin{array}{llll}0.3124813231781984 & 0.4700895455953609 & 0.3850149799133201\end{array}$ $\begin{array}{llll}0.8119412878003731 & 0.4712034126247558 & 0.3848261219065596\end{array}$ $\begin{array}{llll}0.3165808750981282 & 0.9708992052738443 & 0.5351717839262199\end{array}$ $\begin{array}{llll}0.8154615002616401 & 0.9710763910440967 & 0.5341806578547083\end{array}$ $\begin{array}{llll}0.2502224817603447 & 0.5002146897905542 & 0.0599699325556118\end{array}$ $\begin{array}{llll}0.7500842632287779 & 0.5003027304520880 & 0.0600281797887668\end{array}$ $\begin{array}{llll}0.2494979060657594 & 0.0004053538629236 & 0.2101471233643208\end{array}$ $\begin{array}{llll}0.7494759331351036 & 0.0003185752990724 & 0.2101141808001319\end{array}$ $\begin{array}{llll}0.2497058514119623 & 0.4992356690028851 & 0.3587272684366951\end{array}$ $\begin{array}{llll}0.7490014571576590 & 0.5003843640186307 & 0.3585738626733326\end{array}$ $\begin{array}{llll}0.2530532241211002 & 0.0005117865329725 & 0.5090642069511185\end{array}$ $\begin{array}{llll}0.7532645580174264 & 0.0001318749082487 & 0.5077462208873204\end{array}$ $\begin{array}{llll}0.3123879755200781 & 0.5292638696324432 & 0.0335258211060759\end{array}$ $\begin{array}{llll}0.8121588091041880 & 0.5292944013963647 & 0.0335677175501266\end{array}$ $\begin{array}{llll}0.3123520572648920 & 0.0297852639092120 & 0.1839150145808070\end{array}$ $\begin{array}{llll}0.8122389027187322 & 0.0295971572055855 & 0.1838461985876203\end{array}$ $\begin{array}{llll}0.3119151904645225 & 0.5282618705957358 & 0.3323252767965254\end{array}$ $\begin{array}{llll}0.8111546860649125 & 0.5294557584506422 & 0.3321625091834176\end{array}$ $\begin{array}{llll}0.3143174938369785 & 0.0302946008115762 & 0.4825297642531510\end{array}$ $\begin{array}{llll}0.8157124508928232 & 0.0298417553266592 & 0.4814838957145121\end{array}$ $\begin{array}{llll}0.4376156840319805 & 0.9105171952752671 & 0.0688560228417456\end{array}$ $\begin{array}{llll}0.9376407564901016 & 0.9103696593049917 & 0.0689035861225682\end{array}$ $\begin{array}{llll}0.4365226311099061 & 0.4104778529646913 & 0.2190149595682744\end{array}$ $\begin{array}{llll}0.9364675378192554 & 0.4103576951969060 & 0.2189041541782953\end{array}$ $\begin{array}{llll}0.4361884044509745 & 0.9102209330296598 & 0.3673193441644511\end{array}$ $\begin{array}{llll}0.9374289218215850 & 0.9105752540285029 & 0.3673263219501280\end{array}$ $\begin{array}{lllll}0.4397617188326418 & 0.4105217275686278 & 0.5163863406467850\end{array}$ $\begin{array}{llll}0.9405552887234165 & 0.4085751180373413 & 0.5187659977260921\end{array}$ $\begin{array}{llll}0.4371024984068733 & 0.0891035804455143 & 0.0514044937298334\end{array}$ $\begin{array}{llll}0.9371045059996194 & 0.0889934903001493 & 0.0514663823499438\end{array}$ $\begin{array}{lllll}0.4367913723226048 & 0.5890658874243837 & 0.2015672898729567\end{array}$ $\begin{array}{lllll}0.9368506989856126 & 0.5890293948622002 & 0.2014823226755754\end{array}$ $\begin{array}{lllll}0.4358302488247916 & 0.0889803064382501 & 0.3499677817749027\end{array}$ $\begin{array}{lllll}0.9368496045493893 & 0.0894824757742664 & 0.3501592152964976\end{array}$ $\begin{array}{llll}0.4385255813907048 & 0.5889995736795155 & 0.4988348802848641\end{array}$ $\begin{array}{lllll}0.9403316700596583 & 0.5871385845219612 & 0.5013206554066234\end{array}$ $\begin{array}{lllll}0.5628166215219956 & 0.0293112949048637 & 0.0863275756445063\end{array}$ $\begin{array}{llll}0.0628494024119739 & 0.0292466474057835 & 0.0863509364682363\end{array}$ $\begin{array}{llll}0.5617095693645779 & 0.5292703004119769 & 0.2366653563980232\end{array}$ $\begin{array}{llll}0.0616411256225868 & 0.5290476572366780 & 0.2365924532647862\end{array}$ $\begin{array}{llll}0.5617807360975385 & 0.0290594866024592 & 0.3847264175134836\end{array}$ $\begin{array}{llll}0.0626114667160805 & 0.0292968140347056 & 0.3849558370226609\end{array}$ $\begin{array}{llll}0.5643655485927898 & 0.5300624551396721 & 0.5337408318927918\end{array}$ $\begin{array}{llll}0.0676225358117063 & 0.5264370276478335 & 0.5357416315384609\end{array}$ $\begin{array}{llll}0.5001436883478365 & 0.9999175020170935 & 0.0600402076001569\end{array}$ $\begin{array}{llll}0.0001277083809185 & 0.9997923683819600 & 0.0600809492240238\end{array}$ $\begin{array}{llll}0.4993507682922510 & 0.4997153529133630 & 0.2103332503166210\end{array}$ 
$\begin{array}{llll}0.9993493180866160 & 0.4996124690546098 & 0.2102411870174018\end{array}$ $\begin{array}{llll}0.4986858326602240 & 0.9996306574172632 & 0.3585312272093398\end{array}$ $\begin{array}{llll}0.9998064688305898 & 0.0001565570162515 & 0.3586714710186063\end{array}$ $\begin{array}{llll}0.5018894021670565 & 0.5001300251783356 & 0.5074842180114472\end{array}$ $\begin{array}{llll}0.0032282393882921 & 0.4976238681711836 & 0.5097316265928677\end{array}$ $\begin{array}{llll}0.5622802515100095 & 0.9708756344852750 & 0.0335956688146017\end{array}$ $\begin{array}{llll}0.0622122726855590 & 0.9707854065497138 & 0.0336231343746585\end{array}$ $\begin{array}{llll}0.5620942316630289 & 0.4702834230016245 & 0.1840678209661782\end{array}$ $\begin{array}{llll}0.0621712782114803 & 0.4702131078046191 & 0.1839938097735809\end{array}$ $\begin{array}{llll}0.5608222923313225 & 0.9704450565221677 & 0.3321218883191761\end{array}$ $\begin{array}{llll}0.0620255615411610 & 0.9712198581324700 & 0.3322620426875573\end{array}$ $\begin{array}{llll}0.5644210058182573 & 0.4709775642726710 & 0.4811768040589048\end{array}$ $\begin{array}{llll}0.0637236809887451 & 0.4681426569182530 & 0.4830146079042557\end{array}$ $\begin{array}{llll}0.5345966211340254 & 0.4523117140543028 & 0.6142962960989563\end{array}$ $\begin{array}{llll}0.4772686272334491 & 0.5537114870849779 & 0.6177695616837109\end{array}$ $\begin{array}{llll}0.4889811094942059 & 0.6017590420654484 & 0.6450420672006235\end{array}$ $\begin{array}{llll}0.5529114425243643 & 0.5695114634963222 & 0.6659271996388535\end{array}$ $\begin{array}{llll}0.6105377883558365 & 0.4842860053867603 & 0.6617034768419818\end{array}$ $\begin{array}{llll}0.6021640871018884 & 0.4293506177597037 & 0.6369600444412398\end{array}$ $\begin{array}{llll}0.0686267141255593 & 0.3626741490524293 & 0.0149230025077095\end{array}$ $\begin{array}{llll}0.5687013459285595 & 0.3623625598275755 & 0.0150349164614636\end{array}$ $\begin{array}{llll}0.0675026202064927 & 0.8642885834682349 & 0.1653816217247303\end{array}$ $\begin{array}{llll}0.5674054174555779 & 0.8640268596650933 & 0.1654323680507362\end{array}$ $\begin{array}{llll}0.0662484565912485 & 0.3635323035061219 & 0.3144104412848526\end{array}$ $\begin{array}{llll}0.5657046476080334 & 0.3639751851489155 & 0.3143221823719552\end{array}$ $\begin{array}{llll}0.0709222797735768 & 0.8647741642541648 & 0.4641707895883994\end{array}$ $\begin{array}{llll}0.5704496257407823 & 0.8646816023212210 & 0.4629946252251639\end{array}$ $\begin{array}{llll}0.0680614312720366 & 0.6361758981046092 & 0.1048512315172909\end{array}$ $\begin{array}{llll}0.5679184994452739 & 0.6361673148035257 & 0.1049404452420789\end{array}$ $\begin{array}{llll}0.0655505156447312 & 0.1359301562307157 & 0.2544170463389277\end{array}$ $\begin{array}{llll}0.5657719031907468 & 0.1359974221346718 & 0.2544198937414102\end{array}$ $\begin{array}{lllll}0.0675243227876873 & 0.6354587728658408 & 0.4034322539612562\end{array}$ $\begin{array}{llll}0.5665444082408135 & 0.6366647928625673 & 0.4031527915823377\end{array}$ $\begin{array}{llll}0.0728869334483089 & 0.1360616693629313 & 0.5550770339839934\end{array}$ $\begin{array}{llll}0.5737557048737288 & 0.1368642204545101 & 0.5536759344348061\end{array}$ $\begin{array}{llll}0.4340172947812638 & 0.3508741266189413 & 0.1004796554410230\end{array}$ $\begin{array}{llll}0.9341673880011617 & 0.3510715372248243 & 0.1004786865357971\end{array}$ $\begin{array}{llll}0.4330501337330689 & 0.8508962254092526 & 0.2505473827917514\end{array}$ $\begin{array}{llll}0.9331914500667965 & 0.8510419543476866 & 0.2505291645809035\end{array}$ $\begin{array}{llll}0.4342554188450017 & 0.3503842114742112 & 0.3990737160804733\end{array}$ $\begin{array}{llll}0.9339132745882333 & 0.3514222040643031 & 0.3987069206228764\end{array}$ $\begin{array}{lllll}0.4389236818322247 & 0.8515353563185081 & 0.5491949914794449\end{array}$ $\begin{array}{llll}0.9353163054384666 & 0.8506259137391455 & 0.5491227665124266\end{array}$ $\begin{array}{llll}0.4330968288781701 & 0.6492825260653059 & 0.0187789001718809\end{array}$ $\begin{array}{lllll}0.9329518647054711 & 0.6491718884571090 & 0.0187848652566129\end{array}$ $\begin{array}{llll}0.4337067599516718 & 0.1498479621872523 & 0.1699437999220665\end{array}$ $\begin{array}{llll}0.9334493807554303 & 0.1497569283504816 & 0.1698140006911095\end{array}$ $\begin{array}{llll}0.4328957766969760 & 0.6484328935560123 & 0.3180337417109041\end{array}$ $\begin{array}{llll}0.9330912663226413 & 0.6487345370815485 & 0.3179682516158790\end{array}$ $\begin{array}{llll}0.4359567433456362 & 0.1490938714308396 & 0.4679202785879418\end{array}$ $\begin{array}{llll}0.9361714504591534 & 0.1508866717677568 & 0.4674648183131368\end{array}$ $\begin{array}{llll}0.6839251244100911 & 0.1494039918660536 & 0.1005304103891107\end{array}$ $\begin{array}{lllll}0.1839433712757678 & 0.1493199661975571 & 0.1005280102352777\end{array}$ $\begin{array}{llll}0.6830013928389865 & 0.6497086450084461 & 0.2505885487572622\end{array}$ $\begin{array}{llll}0.1831478793092010 & 0.6491751310044714 & 0.2505641671030844\end{array}$ $\begin{array}{llll}0.6838928441281155 & 0.1487603171225874 & 0.3985128009217723\end{array}$ 
$\begin{array}{llll}0.1839693009535519 & 0.1493843583251224 & 0.3990575418586875\end{array}$ $\begin{array}{llll}0.6875713217445915 & 0.6480443589741425 & 0.5477417576064210\end{array}$ $\begin{array}{llll}0.1848493984851680 & 0.6510326152972808 & 0.5499660339013820\end{array}$ $\begin{array}{llll}0.6831220179486174 & 0.8510958861162746 & 0.0188471143148753\end{array}$ $\begin{array}{llll}0.1831121231751750 & 0.8511036209753419 & 0.0188534753227674\end{array}$ $\begin{array}{llll}0.6834805918557958 & 0.3503955216850665 & 0.1700091943541063\end{array}$ $\begin{array}{llll}0.1835870807610275 & 0.3503190364294309 & 0.1699623234983182\end{array}$ $\begin{array}{llll}0.6829674214961771 & 0.8513045880891157 & 0.3179728738242142\end{array}$ $\begin{array}{llll}0.1831513968637822 & 0.8512385884764158 & 0.3179906918819739\end{array}$ $\begin{array}{llll}0.6856294725562494 & 0.3506515698286402 & 0.4671586474637414\end{array}$ $\begin{array}{lllll}0.1852808832871400 & 0.3497663972186199 & 0.4680506711084246\end{array}$ $\begin{array}{llll}0.3179233352776551 & 0.8639555620773164 & 0.1049107759436636\end{array}$ $\begin{array}{lllll}0.8179152606559995 & 0.8638919380600631 & 0.1049501924707003\end{array}$ $\begin{array}{llll}0.3154597628946091 & 0.3639897411744494 & 0.2545694881328203\end{array}$ $\begin{array}{llll}0.8155280298435854 & 0.3639252789186979 & 0.2545192623622430\end{array}$ $\begin{array}{llll}0.3162919617910249 & 0.8635589437882994 & 0.4032469558810602\end{array}$ $\begin{array}{llll}0.8178631503138895 & 0.8629431107813148 & 0.4032140116636824\end{array}$ $\begin{array}{llll}0.3222748831841996 & 0.3635832602246166 & 0.5530202312791658\end{array}$ $\begin{array}{llll}0.8307081270511297 & 0.3578506207404786 & 0.5572377388207770\end{array}$ $\begin{array}{llll}0.3187858581784607 & 0.1372921897760120 & 0.0149027021754447\end{array}$ $\begin{array}{llll}0.8187957563515662 & 0.1372017069788454 & 0.0149688934193143\end{array}$ $\begin{array}{llll}0.3174209076271805 & 0.6354201595425550 & 0.1654384465193983\end{array}$ $\begin{array}{llll}0.8174744408952012 & 0.6354952952967370 & 0.1653738633417803\end{array}$ $\begin{array}{llll}0.3155173319667559 & 0.1358947165158311 & 0.3142137172612420\end{array}$ $\begin{array}{llll}0.8162792909550632 & 0.1361893034319230 & 0.3144443673580506\end{array}$ $\begin{array}{llll}0.3178899106951233 & 0.6351947977048035 & 0.4631528146756022\end{array}$ $\begin{array}{llll}0.8228316570881720 & 0.6347120872569045 & 0.4646057989523804\end{array}$ $\begin{array}{llll}0.0908383712672688 & 0.2797444107853740 & 0.0221148052350714\end{array}$ $\begin{array}{llll}0.5910410267096283 & 0.2794897876652301 & 0.0222535858158054\end{array}$ $\begin{array}{lllll}0.0894016874735920 & 0.7810411096695005 & 0.1722367002503918\end{array}$ $\begin{array}{llll}0.5892331854727518 & 0.7808124230018981 & 0.1723461847608967\end{array}$ $\begin{array}{lllll}0.0887412971730311 & 0.2802400238352196 & 0.3210507006697728\end{array}$ $\begin{array}{lllll}0.5882784015762882 & 0.2808121047051297 & 0.3210566814175344\end{array}$ $\begin{array}{llll}0.0925425152915860 & 0.7814892858092831 & 0.4710270469174219\end{array}$ $\begin{array}{llll}0.5932700054611665 & 0.7813094982716634 & 0.4694402358135483\end{array}$ $\begin{array}{lllll}0.1210537884671893 & 0.4433697005623336 & 0.0288483205908332\end{array}$ $\begin{array}{lllll}0.6208869605571016 & 0.4431654158854606 & 0.0289662071833049\end{array}$ $\begin{array}{lllll}0.1189585029357435 & 0.9439721387535397 & 0.1794926559844973\end{array}$ $\begin{array}{llll}0.6189446560142666 & 0.9438005617905110 & 0.1794724390912081\end{array}$ $\begin{array}{llll}0.1178063344175140 & 0.4431647563549383 & 0.3284907532445036\end{array}$ $\begin{array}{lllll}0.6171647735770706 & 0.4438772041487757 & 0.3283280671757182\end{array}$ $\begin{array}{llll}0.1223587673195944 & 0.9444364895398722 & 0.4784295586158432\end{array}$ $\begin{array}{llll}0.6219893798641982 & 0.9442265398200639 & 0.4772199048579630\end{array}$ $\begin{array}{llll}0.0087871047479187 & 0.3920924574636220 & 0.9976338292326328\end{array}$ $\begin{array}{llll}0.5089113528260089 & 0.3916007543087164 & 0.9977032131539052\end{array}$ $\begin{array}{llll}0.0074938475848259 & 0.8942405200510992 & 0.1481056884281072\end{array}$ $\begin{array}{llll}0.5073896208304877 & 0.8938805972395268 & 0.1481472158382638\end{array}$ $\begin{array}{llll}0.0055111771313578 & 0.3935558208072824 & 0.2973576326935631\end{array}$ $\begin{array}{lllll}0.5049568085176253 & 0.3937169183466672 & 0.2972294668784534\end{array}$ $\begin{array}{llll}0.0113602363146762 & 0.8950459694154751 & 0.4468172900349724\end{array}$ $\begin{array}{llll}0.5094918039811648 & 0.8949496556777102 & 0.4460598753620850\end{array}$ $\begin{array}{llll}0.0897095587293475 & 0.7194266118567216 & 0.0979130534803793\end{array}$ $\begin{array}{llll}0.5893808212161612 & 0.7194263901586745 & 0.0979724338781675\end{array}$ $\begin{array}{llll}0.0882233639706705 & 0.2191835022716959 & 0.2477971941878931\end{array}$ $\begin{array}{llll}0.5884720918164088 & 0.2192452783267867 & 0.2478050325722821\end{array}$ $\begin{array}{llll}0.0892066491513214 & 0.7186492199273545 & 0.3964640017784520\end{array}$ 
$\begin{array}{llll}0.5887968164920256 & 0.7198312321618358 & 0.3963094890625997\end{array}$ $\begin{array}{llll}0.0938208614473231 & 0.2193278218553518 & 0.5478786960302272\end{array}$ $\begin{array}{llll}0.5960602300498161 & 0.2197285170011166 & 0.5465821267368092\end{array}$ $\begin{array}{lllll}0.1198216080082976 & 0.5564174926999964 & 0.0907567220333728\end{array}$ $\begin{array}{llll}0.6197031468672520 & 0.5564634573763259 & 0.0908185393499911\end{array}$ $\begin{array}{llll}0.1172242313537537 & 0.0562132569582551 & 0.2403192294359389\end{array}$ $\begin{array}{lllll}0.6173370525683393 & 0.0562670275133166 & 0.2403113627207066\end{array}$ $\begin{array}{lllll}0.1189119826812216 & 0.5556278839951174 & 0.3892850104590223\end{array}$ $\begin{array}{llll}0.6182641789570491 & 0.5567831994254701 & 0.3891645594594483\end{array}$ $\begin{array}{llll}0.1253212338364484 & 0.0561712155109100 & 0.5408393150464960\end{array}$ $\begin{array}{llll}0.6248305809546952 & 0.0560710480043843 & 0.5391142734370518\end{array}$ $\begin{array}{llll}0.0081943073231447 & 0.6061580052371426 & 0.1221415941666887\end{array}$ $\begin{array}{llll}0.5082285631706561 & 0.6060730892853136 & 0.1222690532261969\end{array}$ $\begin{array}{lllll}0.0046493030463954 & 0.1059174414018967 & 0.2714051425753067\end{array}$ $\begin{array}{llll}0.5048585150459139 & 0.1060256705811309 & 0.2714127383434861\end{array}$ $\begin{array}{llll}0.0078072552594002 & 0.6055200363894763 & 0.4207786662378428\end{array}$ $\begin{array}{llll}0.5060818669658884 & 0.6068527714116505 & 0.4202984216848851\end{array}$ $\begin{array}{lllll}0.0142561737557269 & 0.1058074853669534 & 0.5726131414002910\end{array}$ $\begin{array}{lllll}0.5151340453999674 & 0.1068886288194011 & 0.5712295396982865\end{array}$ $\begin{array}{lllll}0.4112601332574000 & 0.3747685354317176 & 0.1248010084171944\end{array}$ $\begin{array}{lllll}0.9112867088190484 & 0.3747570543616797 & 0.1248059835694267\end{array}$ $\begin{array}{llll}0.4093783035498728 & 0.8734444043412232 & 0.2749105647213323\end{array}$ $\begin{array}{llll}0.9096584708080875 & 0.8737333363430888 & 0.2748922751335657\end{array}$ $\begin{array}{llll}0.4118373011132492 & 0.3742610335910308 & 0.4234271836247698\end{array}$ $\begin{array}{llll}0.9116479449550723 & 0.3749482573758438 & 0.4230948453048375\end{array}$ $\begin{array}{llll}0.4158003056598605 & 0.8742234923738035 & 0.5735362483773728\end{array}$ $\begin{array}{llll}0.9097158246907658 & 0.8728889186488108 & 0.5732880451195091\end{array}$ $\begin{array}{llll}0.3822592708058530 & 0.3973873445806658 & 0.0767587930461706\end{array}$ $\begin{array}{lllll}0.8823862803141859 & 0.3977268873959579 & 0.0767748566546219\end{array}$ $\begin{array}{llll}0.3816505877951687 & 0.8980849890291907 & 0.2269236092078781\end{array}$ $\begin{array}{llll}0.8817345528675106 & 0.8981276409971771 & 0.2268937779824751\end{array}$ $\begin{array}{llll}0.3820397235900371 & 0.3967555442270900 & 0.3754435432082273\end{array}$ $\begin{array}{llll}0.8813439507670523 & 0.3978695926580814 & 0.3751519201666419\end{array}$ $\begin{array}{llll}0.3866716457052479 & 0.8985590215609426 & 0.5254555235048060\end{array}$ $\begin{array}{llll}0.8862753254705992 & 0.8986247266550307 & 0.5250047737816239\end{array}$ $\begin{array}{lllll}0.4950805237638675 & 0.2927252167327419 & 0.0919368120829887\end{array}$ $\begin{array}{lllll}0.9953269440827210 & 0.2930652758596504 & 0.0919063354587785\end{array}$ $\begin{array}{llll}0.4948060446591733 & 0.7936004656689886 & 0.2419290417738077\end{array}$ $\begin{array}{llll}0.9948799007779573 & 0.7936569120239256 & 0.2419172976282510\end{array}$ $\begin{array}{llll}0.4952979290367100 & 0.2923058631893961 & 0.3904496871660506\end{array}$ $\begin{array}{llll}0.9950788823871339 & 0.2936522958225232 & 0.3899807462493897\end{array}$ $\begin{array}{llll}0.5006705745713542 & 0.7944098889760627 & 0.5404146054615500\end{array}$ $\begin{array}{llll}0.9974555264940259 & 0.7930172037268028 & 0.5408530913202966\end{array}$ $\begin{array}{llll}0.4084239335799674 & 0.6266923460538556 & 0.9945690886059344\end{array}$ $\begin{array}{llll}0.9081347843585948 & 0.6265298091380419 & 0.9945912748813295\end{array}$ $\begin{array}{llll}0.4116693757172784 & 0.1256781310913738 & 0.1455759340939183\end{array}$ $\begin{array}{llll}0.9112905134814506 & 0.1257006187111508 & 0.1454471863719248\end{array}$ $\begin{array}{llll}0.4093904532985350 & 0.6251598252523874 & 0.2936998761434467\end{array}$ $\begin{array}{lllll}0.9098695858682861 & 0.6253078291249508 & 0.2936212072935097\end{array}$ $\begin{array}{llll}0.4119959025573819 & 0.1252375811105566 & 0.4436790035578498\end{array}$ $\begin{array}{llll}0.9128846176529315 & 0.1280046455073070 & 0.4430910505702529\end{array}$ $\begin{array}{llll}0.3830545097240942 & 0.6017570392935461 & 0.0427922813312863\end{array}$ $\begin{array}{llll}0.8828450679699984 & 0.6017739889040873 & 0.0428176069746068\end{array}$ $\begin{array}{llll}0.3815586277034851 & 0.1033984302724887 & 0.1935293584666989\end{array}$ $\begin{array}{llll}0.8814853352018083 & 0.1032368953434964 & 0.1934223873767737\end{array}$ $\begin{array}{llll}0.3815544668664604 & 0.6018134243129455 & 0.3417399422747363\end{array}$ 
$\begin{array}{llll}0.8810977893406259 & 0.6026549618207601 & 0.3416275787236374\end{array}$ $\begin{array}{llll}0.3848966944327082 & 0.1030162403746386 & 0.4918212329871786\end{array}$ $\begin{array}{lllll}0.8851667222740606 & 0.1032884418474778 & 0.4910806089458178\end{array}$ $\begin{array}{llll}0.4949751536643216 & 0.7069817981155777 & 0.0271977866945181\end{array}$ $\begin{array}{llll}0.9949485333814595 & 0.7067922110414439 & 0.0271721800367792\end{array}$ $\begin{array}{llll}0.4943738498194747 & 0.2083452184752752 & 0.1785547101209302\end{array}$ $\begin{array}{lllll}0.9941683966648953 & 0.2082024246806022 & 0.1784170915534583\end{array}$ $\begin{array}{llll}0.4944913833804495 & 0.7061646959127804 & 0.3265061930199132\end{array}$ $\begin{array}{llll}0.9948451329576264 & 0.7062336333888539 & 0.3264727184829386\end{array}$ $\begin{array}{llll}0.4977667243311633 & 0.2069119993469253 & 0.4761939592513273\end{array}$ $\begin{array}{lllll}0.9973165881143904 & 0.2088670752650841 & 0.4760719816029177\end{array}$ $\begin{array}{llll}0.6612207077819190 & 0.1253186951285494 & 0.1248404094607851\end{array}$ $\begin{array}{llll}0.1612704375683378 & 0.1252759195441684 & 0.1248421820018292\end{array}$ $\begin{array}{lllll}0.6594804034510694 & 0.6273808609014481 & 0.2749837980036757\end{array}$ $\begin{array}{llll}0.1596667825564106 & 0.6266499270203851 & 0.2749456372424710\end{array}$ $\begin{array}{llll}0.6620835523457000 & 0.1248926342548159 & 0.4229124710368928\end{array}$ $\begin{array}{llll}0.1615545357681489 & 0.1255185531697476 & 0.4234107167103976\end{array}$ $\begin{array}{llll}0.6689374036208989 & 0.6205580325749022 & 0.5720246457048106\end{array}$ $\begin{array}{llll}0.1585361758693214 & 0.6314539855786385 & 0.5742401892137066\end{array}$ $\begin{array}{lllll}0.6322058703069391 & 0.1028295226848986 & 0.0768054647260547\end{array}$ $\begin{array}{llll}0.1321212699215882 & 0.1028329775719380 & 0.0768118259525314\end{array}$ $\begin{array}{llll}0.6316084852153686 & 0.6022036111715795 & 0.2270218929409062\end{array}$ $\begin{array}{llll}0.1315667164823043 & 0.6019774490597765 & 0.2269679813686417\end{array}$ $\begin{array}{llll}0.6310731871006990 & 0.1023990852723563 & 0.3749958588364027\end{array}$ $\begin{array}{lllll}0.1319455358502409 & 0.1028763363847097 & 0.3754085024641390\end{array}$ $\begin{array}{llll}0.6329802464095476 & 0.6044999157628719 & 0.5241919105048852\end{array}$ $\begin{array}{llll}0.1374828166474086 & 0.5990157911455145 & 0.5261329443996678\end{array}$ $\begin{array}{llll}0.7448899478618449 & 0.2077010122507044 & 0.0920100317462909\end{array}$ $\begin{array}{llll}0.2449400003116515 & 0.2075684025370654 & 0.0919948404768845\end{array}$ $\begin{array}{llll}0.7446167567875349 & 0.7070522138294336 & 0.2418953849309084\end{array}$ $\begin{array}{llll}0.2448577281277758 & 0.7064555702648838 & 0.2419014044198284\end{array}$ $\begin{array}{llll}0.7447298483046285 & 0.2068242282920230 & 0.3897578984314338\end{array}$ $\begin{array}{llll}0.2449226150468216 & 0.2075687769764246 & 0.3904392595069328\end{array}$ $\begin{array}{llll}0.7470672770731170 & 0.7080951650693402 & 0.5391586390356515\end{array}$ $\begin{array}{llll}0.2460224331062131 & 0.7087894876345765 & 0.5412195412392043\end{array}$ $\begin{array}{llll}0.3393379518716625 & 0.7807111323688735 & 0.0979195541832439\end{array}$ $\begin{array}{llll}0.8393420896597229 & 0.7806432501798608 & 0.0979578379521710\end{array}$ $\begin{array}{llll}0.3382620639798890 & 0.2808049808197652 & 0.2479187130681192\end{array}$ $\begin{array}{lllll}0.8382233225452461 & 0.2807069039501208 & 0.2478719448296227\end{array}$ $\begin{array}{llll}0.3386023328985583 & 0.7803747808042151 & 0.3964488886641668\end{array}$ $\begin{array}{lllll}0.8396739098481990 & 0.7799233404904437 & 0.3960966329376345\end{array}$ $\begin{array}{llll}0.3477664569856648 & 0.2804478700497260 & 0.5470060190097169\end{array}$ $\begin{array}{llll}0.8465181511930656 & 0.2757984279906963 & 0.5479787066145029\end{array}$ $\begin{array}{llll}0.6584317843239206 & 0.8738297263011587 & 0.9946474496980957\end{array}$ $\begin{array}{llll}0.1584033862443728 & 0.8739102588639400 & 0.9946638625679405\end{array}$ $\begin{array}{llll}0.6611119871606038 & 0.3743329674218248 & 0.1456492804047042\end{array}$ $\begin{array}{llll}0.1613342305388623 & 0.3743511515723526 & 0.1456005666488898\end{array}$ $\begin{array}{lllll}0.6598743983955166 & 0.8747456619548091 & 0.2936185347575408\end{array}$ $\begin{array}{lllll}0.1597290794212026 & 0.8745279234348602 & 0.2936511370587473\end{array}$ $\begin{array}{llll}0.6624300682449837 & 0.3735798876559797 & 0.4427711150490309\end{array}$ $\begin{array}{llll}0.1617623095831262 & 0.3747666221735382 & 0.4438685877438476\end{array}$ $\begin{array}{lllll}0.6328910233907794 & 0.8983594955686367 & 0.0428715599880832\end{array}$ $\begin{array}{llll}0.1328371129019470 & 0.8982376877349876 & 0.0428857582224498\end{array}$ $\begin{array}{llll}0.6314049031706325 & 0.3967405839425962 & 0.1936376594685366\end{array}$ $\begin{array}{llll}0.1314730413432361 & 0.3966752842744691 & 0.1935803297977135\end{array}$ $\begin{array}{llll}0.6308771991579328 & 0.8973883612826098 & 0.3416199152468675\end{array}$ 
$\begin{array}{llll}0.1316980904452729 & 0.8977528323784103 & 0.3416918546358496\end{array}$ $\begin{array}{llll}0.6343911900724633 & 0.3979655654834390 & 0.4907423373016955\end{array}$ $\begin{array}{llll}0.1342144175437044 & 0.3949765790406226 & 0.4920599133385519\end{array}$ $\begin{array}{llll}0.7451102466058804 & 0.7934866777717529 & 0.0272475627290718\end{array}$ $\begin{array}{lllll}0.2451299536938207 & 0.7935039390885547 & 0.0272363429042630\end{array}$ $\begin{array}{llll}0.7444778921661692 & 0.2922013569218272 & 0.1785904314950750\end{array}$ $\begin{array}{llll}0.2444816641701675 & 0.2920390212522780 & 0.1785558142725187\end{array}$ $\begin{array}{llll}0.7446679285691399 & 0.7937965633224586 & 0.3265074166589479\end{array}$ $\begin{array}{llll}0.2447557269074930 & 0.7935679615906168 & 0.3264843411180383\end{array}$ $\begin{array}{llll}0.7468657748006879 & 0.2927265398201215 & 0.4757598592974427\end{array}$ $\begin{array}{llll}0.2467132431533413 & 0.2912935656971218 & 0.4761691296565235\end{array}$ $\begin{array}{llll}0.3697754618211231 & 0.9437018436352431 & 0.0908219708772057\end{array}$ $\begin{array}{llll}0.8697717184546335 & 0.9435899248912170 & 0.0908587036370925\end{array}$ $\begin{array}{llll}0.3671546343341044 & 0.4438160689931550 & 0.2405462222600497\end{array}$ $\begin{array}{llll}0.8671399155442375 & 0.4437087245167208 & 0.2404400990833244\end{array}$ $\begin{array}{llll}0.3677199993345527 & 0.9434215097299299 & 0.3891033800000961\end{array}$ $\begin{array}{lllll}0.8693238287801759 & 0.9431432483739932 & 0.3892839787503708\end{array}$ $\begin{array}{llll}0.3699897829293593 & 0.4436435976951294 & 0.5377342630235753\end{array}$ $\begin{array}{lllll}0.8804502656180481 & 0.4394210182715174 & 0.5428941308643039\end{array}$ $\begin{array}{llll}0.2582211298222695 & 0.8940634519040982 & 0.1222363283560125\end{array}$ $\begin{array}{llll}0.7582277182826636 & 0.8939973672456583 & 0.1222817041667967\end{array}$ $\begin{array}{llll}0.2543945612477489 & 0.3938398346595819 & 0.2715280514141625\end{array}$ $\begin{array}{llll}0.7545988564550702 & 0.3938430169185502 & 0.2715150211887542\end{array}$ $\begin{array}{llll}0.2559704173516951 & 0.8934710667967696 & 0.4204195670437629\end{array}$ $\begin{array}{llll}0.7579610463522132 & 0.8924042384603454 & 0.4205853037501481\end{array}$ $\begin{array}{llll}0.2613530629861856 & 0.3930726656143223 & 0.5699918618044022\end{array}$ $\begin{array}{llll}0.7792694807269638 & 0.3857082647610401 & 0.5769098986981094\end{array}$ $\begin{array}{lllll}0.3409921158356411 & 0.2202331831084168 & 0.0220838566216537\end{array}$ $\begin{array}{lllll}0.8409908508935955 & 0.2201387472920037 & 0.0221657986705925\end{array}$ $\begin{array}{lllll}0.3392575548182841 & 0.7187296357671344 & 0.1722514291125602\end{array}$ $\begin{array}{llll}0.8393451900361959 & 0.7187995267577978 & 0.1721892300349178\end{array}$ $\begin{array}{llll}0.3381009681174411 & 0.2190675491453661 & 0.3209404199721188\end{array}$ $\begin{array}{lllll}0.8388754796732717 & 0.2194317014738804 & 0.3211014148573689\end{array}$ $\begin{array}{llll}0.3394783145023287 & 0.7184670196651692 & 0.4701129643491246\end{array}$ $\begin{array}{lllll}0.8470248519250271 & 0.7178507013841704 & 0.4708558333071262\end{array}$ $\begin{array}{lllll}0.3710386527424714 & 0.0566350172610204 & 0.0288954240199501\end{array}$ $\begin{array}{lllll}0.8709903269123649 & 0.0565396982875817 & 0.0289635563150803\end{array}$ $\begin{array}{llll}0.3687721183737144 & 0.5558328874697364 & 0.1796468015487115\end{array}$ $\begin{array}{llll}0.8688159343392006 & 0.5558666112208469 & 0.1795653314559826\end{array}$ $\begin{array}{llll}0.3668798114437151 & 0.0560226730855088 & 0.3282651864336925\end{array}$ $\begin{array}{lllll}0.8678330713320336 & 0.0564119430031407 & 0.3284911744760806\end{array}$ $\begin{array}{llll}0.3701142470881756 & 0.5556198994911832 & 0.4770349192189174\end{array}$ $\begin{array}{llll}0.8715864494447816 & 0.5546241328600285 & 0.4795219563392429\end{array}$ $\begin{array}{llll}0.2590789676688760 & 0.1078195063832285 & 0.9975815171476141\end{array}$ $\begin{array}{llll}0.7591604918402545 & 0.1077734447044601 & 0.9976156311084544\end{array}$ $\begin{array}{llll}0.2575345617129591 & 0.6053151071303278 & 0.1481464359053151\end{array}$ $\begin{array}{llll}0.7575745466531305 & 0.6054116070369991 & 0.1480843140057518\end{array}$ $\begin{array}{llll}0.2548323121750732 & 0.1061516782849525 & 0.2971025791308960\end{array}$ $\begin{array}{llll}0.7554519509709895 & 0.1062461545247233 & 0.2974078681504499\end{array}$ $\begin{array}{llll}0.2576585143010343 & 0.6052824714856420 & 0.4459282245010782\end{array}$ $\begin{array}{llll}0.7625412457199719 & 0.6048201098131626 & 0.4474196676594352\end{array}$ $\begin{array}{llll}0.5197600772029203 & 0.4015212537715569 & 0.5925781560594759\end{array}$ $\begin{array}{llll}0.4261911235161025 & 0.5837891746903835 & 0.5975500008809738\end{array}$ 
TS structure of hydrogen abstraction reaction on PETN (110) surface Method: PBE

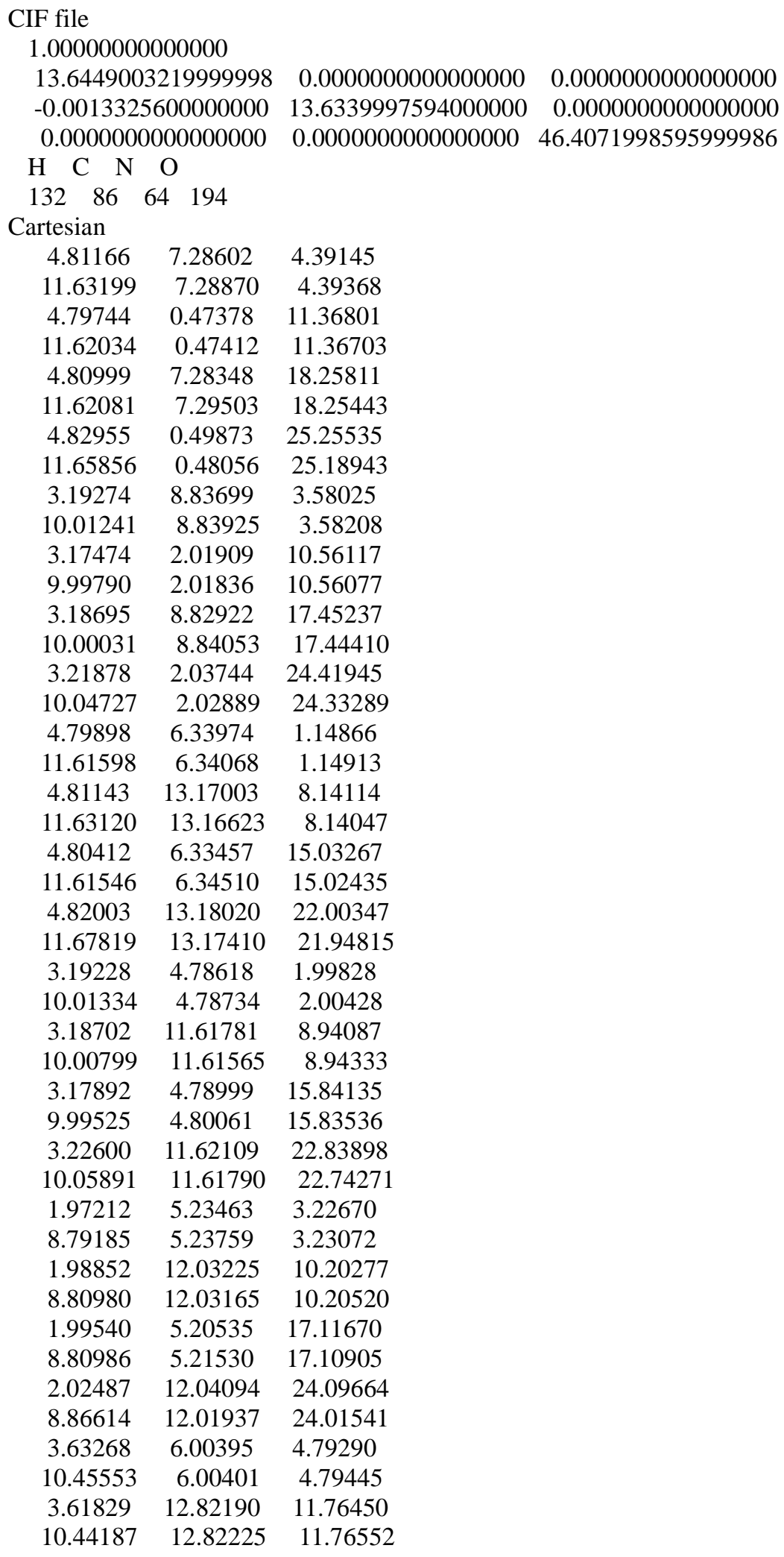




$\begin{array}{ccc}3.63294 & 6.00160 & 18.66686 \\ 10.44506 & 6.01040 & 18.65993 \\ 3.67304 & 12.82308 & 25.64316 \\ 10.48038 & 12.82620 & 25.57842 \\ 1.98997 & 8.41523 & 2.32430 \\ 8.80896 & 8.41625 & 2.32725 \\ 1.99450 & 1.60938 & 9.28134 \\ 8.81707 & 1.60868 & 9.28150 \\ 1.99175 & 8.41206 & 16.18805 \\ 8.80134 & 8.42048 & 16.18446 \\ 1.98450 & 1.58760 & 23.20434 \\ 8.81599 & 1.56662 & 23.12232 \\ 3.62829 & 7.63600 & 0.76444 \\ 10.44658 & 7.63859 & 0.76671 \\ 3.63256 & 0.81534 & 7.73206 \\ 10.45163 & 0.81070 & 7.73096 \\ 3.62171 & 7.61380 & 14.62813 \\ 10.43806 & 7.63034 & 14.62419 \\ 3.63867 & 0.83057 & 21.61910 \\ 10.49512 & 0.81947 & 21.55094 \\ 5.39888 & 12.03559 & 2.32749 \\ 12.22287 & 12.03298 & 2.32961 \\ 5.40656 & 5.20482 & 9.28549 \\ 12.22824 & 5.20549 & 9.28329 \\ 5.38729 & 12.02377 & 16.18614 \\ 12.22632 & 12.03547 & 16.18124 \\ 5.47847 & 5.17321 & 23.22890 \\ 12.19598 & 5.23799 & 23.24306 \\ 7.03656 & 12.81357 & 0.76654 \\ 0.21358 & 12.81227 & 0.76816 \\ 7.04024 & 6.00100 & 7.73490 \\ 0.21866 & 6.00169 & 7.73326 \\ 7.01391 & 12.81235 & 14.62316 \\ 0.21284 & 12.83321 & 14.62509 \\ 7.05250 & 6.11959 & 21.71359 \\ 0.22084 & 5.97362 & 21.63761 \\ 5.38079 & 1.58067 & 3.22908 \\ 12.20297 & 1.57920 & 3.23087 \\ 5.39869 & 8.41760 & 10.20645 \\ 12.22077 & 8.41736 & 10.20484 \\ 5.39205 & 1.60082 & 17.10715 \\ 12.23261 & 1.60656 & 17.11687 \\ 5.32246 & 8.41995 & 24.18790 \\ 12.28035 & 8.38257 & 24.15105 \\ 7.04474 & 0.81296 & 4.79454 \\ 0.22253 & 0.81258 & 4.79562 \\ 7.03049 & 7.63044 & 11.76626 \\ 0.20795 & 7.62900 & 11.76572 \\ 7.03692 & 0.80826 & 18.65593 \\ 0.22284 & 0.80801 & 18.66466 \\ 6.89991 & 7.50095 & 26.08404 \\ 0.29601 & 7.58077 & 25.67541 \\ 8.22185 & 13.16465 & 4.39218 \\ 1.40012 & 13.16460 & 4.39342 \\ 8.20813 & 6.34420 & 11.37050 \\ 1.38549 & 6.34306 & 11.36883\end{array}$




$\begin{array}{ccc}8.21360 & 13.16198 & 18.24378 \\ 1.40044 & 13.16213 & 18.25496 \\ 8.31504 & 6.43598 & 25.33832 \\ 1.46796 & 6.29959 & 25.25292 \\ 6.60218 & 11.61424 & 3.58297 \\ 13.42588 & 11.61381 & 3.58616 \\ 6.58647 & 4.79889 & 10.56683 \\ 13.40852 & 4.79782 & 10.56380 \\ 6.58164 & 11.61295 & 17.45295 \\ 13.42439 & 11.61356 & 17.44098 \\ 6.59030 & 4.81902 & 24.58848 \\ 13.47973 & 4.73395 & 24.38069 \\ 8.20687 & 0.47812 & 1.14853 \\ 1.38373 & 0.47698 & 1.14964 \\ 8.22033 & 7.28119 & 8.14177 \\ 1.39835 & 7.28197 & 8.14108 \\ 8.19212 & 0.46627 & 15.01689 \\ 1.39548 & 0.47903 & 15.03077 \\ 8.22149 & 7.38381 & 22.20719 \\ 1.40674 & 7.25675 & 22.01759 \\ 6.60194 & 2.03134 & 2.00242 \\ 13.42380 & 2.03021 & 2.00406 \\ 6.59649 & 8.83335 & 8.94412 \\ 13.41873 & 8.83315 & 8.94267 \\ 6.57749 & 2.01429 & 15.83300 \\ 13.41427 & 2.02239 & 15.84023 \\ 6.53625 & 8.84784 & 22.94516 \\ 13.45732 & 8.81017 & 22.87535 \\ 6.23085 & 9.20265 & 29.41749 \\ 7.31956 & 8.35590 & 31.48780 \\ 8.63081 & 6.23631 & 31.46734 \\ 8.74463 & 4.85487 & 29.38521 \\ 2.55701 & 5.59959 & 2.37139 \\ 9.37738 & 5.60117 & 2.37529 \\ 2.55235 & 12.41765 & 9.34251 \\ 9.37367 & 12.41601 & 9.34453 \\ 2.55041 & 5.59065 & 16.25075 \\ 9.36546 & 5.60087 & 16.24357 \\ 2.59062 & 12.42285 & 23.23607 \\ 9.42433 & 12.41253 & 23.15516 \\ 2.55955 & 8.03367 & 3.18282 \\ 9.37969 & 8.03524 & 3.18528 \\ 2.54636 & 1.22035 & 10.14777 \\ 9.36898 & 1.21976 & 10.14792 \\ 2.55430 & 8.02817 & 17.04992 \\ 9.36733 & 8.03814 & 17.04474 \\ 2.58256 & 1.22260 & 24.05051 \\ 9.41305 & 1.20864 & 23.97224 \\ 4.26769 & 6.41610 & 3.99817 \\ 11.08912 & 6.41835 & 3.99975 \\ 4.25314 & 13.23862 & 10.97245 \\ 11.07605 & 13.23840 & 10.97258 \\ 4.26473 & 6.41263 & 17.86905 \\ 11.07718 & 6.42391 & 17.86351 \\ 4.30330 & 13.25377 & 24.85396 \\ 11.12337 & 13.23976 & 24.79091 \\ & & \\ & & \end{array}$




\begin{tabular}{ccc}
3.41555 & 6.81582 & 2.77609 \\
10.23572 & 6.81795 & 2.77842 \\
3.40541 & 0.00347 & 9.74821 \\
10.22728 & 0.00206 & 9.74863 \\
3.40881 & 6.80858 & 16.64795 \\
10.22241 & 6.81970 & 16.64166 \\
3.44360 & 0.01215 & 23.63539 \\
10.27605 & 13.63275 & 23.56254 \\
4.26655 & 7.21333 & 1.55156 \\
11.08535 & 7.21497 & 1.55288 \\
4.26303 & 0.40384 & 8.53057 \\
11.08331 & 0.40067 & 8.52924 \\
4.25768 & 7.20401 & 15.42290 \\
11.07275 & 7.21555 & 15.41748 \\
4.28253 & 0.41556 & 22.40521 \\
11.12996 & 0.40510 & 22.34455 \\
5.96911 & 12.41759 & 3.18535 \\
12.79232 & 12.41626 & 3.18746 \\
5.95736 & 5.59611 & 10.15151 \\
12.77961 & 5.59567 & 10.14946 \\
5.94824 & 12.41180 & 17.04719 \\
12.79001 & 12.41593 & 17.04386 \\
5.95648 & 5.58636 & 24.12850 \\
12.83379 & 5.56685 & 24.07501 \\
5.96688 & 1.21710 & 2.37401 \\
12.78891 & 1.21580 & 2.37565 \\
5.96257 & 8.03301 & 9.34585 \\
12.78495 & 8.03271 & 9.34444 \\
5.94726 & 1.21457 & 16.24166 \\
12.78594 & 1.22154 & 16.24961 \\
5.91118 & 8.03877 & 23.34352 \\
12.82992 & 8.00465 & 23.27730 \\
7.67909 & 0.40074 & 3.99935 \\
0.85688 & 0.40030 & 4.00049 \\
7.66500 & 7.21296 & 10.97425 \\
0.84244 & 7.21223 & 10.97336 \\
7.66487 & 0.39615 & 17.85567 \\
0.85503 & 0.39836 & 17.86663 \\
7.65803 & 7.23029 & 24.96654 \\
0.92027 & 7.17258 & 24.87009 \\
6.82614 & 0.00128 & 2.77783 \\
0.00236 & 13.63414 & 2.77943 \\
6.81523 & 6.81355 & 9.75080 \\
13.63772 & 6.81333 & 9.74956 \\
6.80249 & 13.62980 & 16.63970 \\
13.64456 & 0.00313 & 16.64518 \\
6.78602 & 6.83489 & 23.76586 \\
0.04419 & 6.78239 & 23.66116 \\
7.67493 & 13.23797 & 1.55261 \\
0.85211 & 13.23678 & 1.55407 \\
7.67157 & 6.41329 & 8.53229 \\
0.84968 & 6.41374 & 8.53104 \\
7.64966 & 13.23048 & 15.41369 \\
0.84812 & 13.24310 & 15.42040 \\
7.67311 & 6.49045 & 22.53919 \\
0.87096 & 6.38594 & 22.42020 \\
& & \\
\hline
\end{tabular}




\begin{tabular}{ccc}
7.52883 & 6.19848 & 28.20920 \\
6.88209 & 7.55074 & 28.20164 \\
6.77188 & 8.25660 & 29.42121 \\
7.38226 & 7.77544 & 30.56636 \\
8.12067 & 6.55927 & 30.55871 \\
8.19104 & 5.79326 & 29.42243 \\
0.93410 & 4.94955 & 0.68204 \\
7.75805 & 4.94607 & 0.68481 \\
0.92027 & 11.77981 & 7.67207 \\
7.74099 & 11.77838 & 7.67434 \\
0.90579 & 4.95686 & 14.59138 \\
7.72051 & 4.96227 & 14.58704 \\
0.97200 & 11.77948 & 21.55150 \\
7.77809 & 11.77629 & 21.49812 \\
0.92985 & 8.67007 & 4.85834 \\
7.74979 & 8.66955 & 4.86157 \\
0.89853 & 1.85489 & 11.80296 \\
7.72219 & 1.85525 & 11.80465 \\
0.92176 & 8.66437 & 18.72226 \\
7.73419 & 8.67287 & 18.71772 \\
0.98401 & 1.85365 & 25.77020 \\
7.80977 & 1.86136 & 25.67639 \\
5.92264 & 4.78382 & 4.66268 \\
12.74656 & 4.78588 & 4.65925 \\
5.90852 & 11.60121 & 11.62673 \\
12.73450 & 11.60352 & 11.62656 \\
5.92767 & 4.78477 & 18.52902 \\
12.73837 & 4.79237 & 18.51973 \\
6.00155 & 11.66830 & 25.52350 \\
12.75138 & 11.59429 & 25.49416 \\
5.90661 & 8.85780 & 0.87041 \\
12.72870 & 8.85418 & 0.86853 \\
5.92075 & 2.03960 & 7.88217 \\
12.73842 & 2.03675 & 7.87491 \\
5.90594 & 8.84392 & 14.75835 \\
12.73223 & 8.84539 & 14.75729 \\
5.93802 & 2.03872 & 21.72400 \\
12.77347 & 2.05592 & 21.69547 \\
9.33310 & 2.03452 & 4.66287 \\
2.51058 & 2.03449 & 4.66297 \\
9.31869 & 8.85302 & 11.62665 \\
2.49891 & 8.84913 & 11.62679 \\
9.32632 & 2.03293 & 18.49715 \\
2.51285 & 2.03131 & 18.52660 \\
9.42561 & 8.78576 & 25.62684 \\
2.50948 & 8.87638 & 25.53837 \\
9.31980 & 11.59891 & 0.87127 \\
2.49720 & 11.59857 & 0.87193 \\
9.32983 & 4.77896 & 7.88245 \\
2.50802 & 4.77945 & 7.88095 \\
9.31736 & 11.60740 & 14.75628 \\
2.49869 & 11.60526 & 14.75675 \\
9.29349 & 4.84483 & 21.81316 \\
2.52805 & 4.76946 & 21.72524 \\
4.33832 & 11.78329 & 4.86100 \\
11.16071 & 11.78225 & 4.86192 \\
& & \\
\hline
\end{tabular}




$\begin{array}{ccc}4.30865 & 4.96247 & 11.80668 \\ 11.13153 & 4.96212 & 11.80560 \\ 4.31453 & 11.77590 & 18.71681 \\ 11.16034 & 11.76647 & 18.71159 \\ 4.30377 & 4.89680 & 25.76121 \\ 11.35179 & 4.85719 & 25.87077 \\ 4.34755 & 1.86805 & 0.68139 \\ 11.17007 & 1.86778 & 0.68295 \\ 4.33147 & 8.66888 & 7.67332 \\ 11.15436 & 8.66809 & 7.67101 \\ 4.30561 & 1.85299 & 14.58199 \\ 11.13922 & 1.85848 & 14.59319 \\ 4.35764 & 8.62784 & 21.57553 \\ 11.22611 & 8.66152 & 21.57462 \\ 1.23209 & 3.81716 & 1.01470 \\ 8.05827 & 3.81465 & 1.01889 \\ 1.21723 & 10.64534 & 7.99422 \\ 8.03878 & 10.64372 & 7.99511 \\ 1.21217 & 3.82127 & 14.90010 \\ 8.02763 & 3.82813 & 14.89970 \\ 1.27466 & 10.64502 & 21.86858 \\ 8.08363 & 10.64102 & 21.81009 \\ 1.65591 & 6.04532 & 1.32784 \\ 8.47696 & 6.04422 & 1.33014 \\ 1.62306 & 12.86768 & 8.32512 \\ 8.44445 & 12.86598 & 8.32704 \\ 1.61009 & 6.04274 & 15.24435 \\ 8.42516 & 6.05088 & 15.23598 \\ 1.66298 & 12.86876 & 22.21556 \\ 8.48616 & 12.86401 & 22.14644 \\ 0.11833 & 5.35525 & 46.28838 \\ 6.94207 & 5.34910 & 46.29006 \\ 0.10315 & 12.18692 & 6.86808 \\ 6.92251 & 12.18585 & 6.87198 \\ 0.07719 & 5.36633 & 13.80006 \\ 6.89092 & 5.36855 & 13.79502 \\ 0.15743 & 12.18635 & 20.74496 \\ 6.94865 & 12.18427 & 20.70737 \\ 1.22330 & 9.80459 & 4.53337 \\ 8.04114 & 9.80453 & 4.53631 \\ 1.20804 & 2.98946 & 11.49426 \\ 8.03345 & 2.98999 & 11.49839 \\ 1.21825 & 9.79935 & 18.40150 \\ 8.03265 & 9.80761 & 18.39764 \\ 1.26560 & 2.98958 & 25.43516 \\ 8.08402 & 2.98525 & 25.30887 \\ 1.63592 & 7.58238 & 4.20454 \\ 8.45736 & 7.58297 & 4.20772 \\ 1.60253 & 0.76699 & 11.14985 \\ 8.42512 & 0.76722 & 11.15042 \\ 1.62375 & 7.57732 & 18.06575 \\ 8.44143 & 7.58570 & 18.06389 \\ 1.69859 & 0.76653 & 25.10434 \\ 8.53537 & 0.75665 & 25.02849 \\ 0.11528 & 8.26138 & 5.66346 \\ 6.93626 & 8.25949 & 5.66704 \\ & & \end{array}$




$\begin{array}{ccc}0.06774 & 1.44696 & 12.59226 \\ 6.89020 & 1.44701 & 12.59259 \\ 0.10558 & 8.25543 & 19.52603 \\ 6.91482 & 8.26368 & 19.51737 \\ 0.18919 & 1.43852 & 26.58726 \\ 7.02370 & 1.45510 & 26.50519 \\ 5.60952 & 5.11150 & 5.79019 \\ 12.43380 & 5.10950 & 5.78805 \\ 5.58660 & 11.91139 & 12.75708 \\ 12.41598 & 11.91626 & 12.75717 \\ 5.62230 & 5.11457 & 19.65753 \\ 12.42456 & 5.11090 & 19.64973 \\ 5.65925 & 11.96255 & 26.64953 \\ 12.40124 & 11.90439 & 26.61347 \\ 5.21845 & 5.41619 & 3.55935 \\ 12.04113 & 5.42087 & 3.55830 \\ 5.20668 & 12.24204 & 10.52940 \\ 12.02929 & 12.24171 & 10.52957 \\ 5.21403 & 5.41211 & 17.42817 \\ 12.02877 & 5.42614 & 17.42119 \\ 5.27202 & 12.27855 & 24.41284 \\ 12.08413 & 12.24352 & 24.37073 \\ 6.75723 & 3.99060 & 4.26976 \\ 13.58172 & 3.99480 & 4.26310 \\ 6.75062 & 10.81857 & 11.22826 \\ 13.57557 & 10.82025 & 11.22722 \\ 6.76096 & 3.99220 & 18.13085 \\ 13.57699 & 4.00646 & 18.12021 \\ 6.87939 & 10.92732 & 25.12450 \\ 13.59657 & 10.80299 & 25.11520 \\ 5.56765 & 8.55334 & 46.15385 \\ 12.38569 & 8.55115 & 46.15279 \\ 5.61810 & 1.71341 & 6.75104 \\ 12.43331 & 1.70906 & 6.74477 \\ 5.58325 & 8.52726 & 13.62964 \\ 12.41358 & 8.52702 & 13.62782 \\ 5.61669 & 1.70598 & 20.59945 \\ 12.45569 & 1.74448 & 20.56390 \\ 5.22834 & 8.20250 & 1.98429 \\ 12.04959 & 8.20240 & 1.98400 \\ 5.20819 & 1.40659 & 8.97697 \\ 12.02904 & 1.40417 & 8.97216 \\ 5.20755 & 8.20700 & 15.85945 \\ 12.02677 & 8.21333 & 15.85673 \\ 5.23901 & 1.41371 & 22.83443 \\ 12.07687 & 1.40643 & 22.79087 \\ 6.74922 & 9.64550 & 1.26261 \\ 13.57605 & 9.63773 & 1.25881 \\ 6.75148 & 2.83360 & 8.28286 \\ 13.56925 & 2.83184 & 8.27303 \\ 6.74683 & 9.63088 & 15.15087 \\ 13.57392 & 9.63034 & 15.15171 \\ 6.77693 & 2.83129 & 22.10721 \\ 13.60762 & 2.84623 & 22.09512 \\ 9.01890 & 1.70888 & 5.79067 \\ 2.19647 & 1.70892 & 5.79085 \\ & & \end{array}$




$\begin{array}{ccc}8.99571 & 8.54584 & 12.75750 \\ 2.17963 & 8.53709 & 12.75731 \\ 9.03110 & 1.70463 & 19.62919 \\ 2.20351 & 1.70493 & 19.65538 \\ 9.50700 & 8.12388 & 26.63265 \\ 2.16200 & 8.59218 & 26.66448 \\ 8.62983 & 1.40039 & 3.55993 \\ 1.80710 & 1.40025 & 3.56037 \\ 8.61838 & 8.20913 & 10.53016 \\ 1.79564 & 8.20884 & 10.52981 \\ 8.60923 & 1.39823 & 17.40527 \\ 1.80294 & 1.40069 & 17.42628 \\ 8.33593 & 8.41086 & 24.64997 \\ 1.86349 & 8.17501 & 24.42947 \\ 10.16813 & 2.82696 & 4.26931 \\ 3.34559 & 2.82689 & 4.26930 \\ 10.16090 & 9.63486 & 11.22697 \\ 3.33930 & 9.63318 & 11.22753 \\ 10.15275 & 2.82959 & 18.09208 \\ 3.34745 & 2.82274 & 18.12957 \\ 10.06767 & 9.72570 & 25.20942 \\ 3.33451 & 9.67639 & 25.13640 \\ 4.62927 & 10.64831 & 4.53546 \\ 11.45212 & 10.64730 & 4.53657 \\ 4.61866 & 3.82764 & 11.49953 \\ 11.44134 & 3.82714 & 11.49883 \\ 4.61694 & 10.64170 & 18.39950 \\ 11.45955 & 10.63443 & 18.38295 \\ 4.68231 & 3.77226 & 25.48722 \\ 11.56442 & 3.74483 & 25.42365 \\ 8.97902 & 11.90189 & 46.15487 \\ 2.15608 & 11.90215 & 46.15581 \\ 9.02640 & 5.10557 & 6.75163 \\ 2.20432 & 5.10587 & 6.75015 \\ 9.00230 & 11.92773 & 13.62633 \\ 2.17863 & 11.92385 & 13.62787 \\ 8.95883 & 5.19030 & 20.69723 \\ 2.20472 & 5.10826 & 20.60323 \\ 8.63898 & 12.25088 & 1.98521 \\ 1.81647 & 12.24990 & 1.98635 \\ 8.61775 & 5.41118 & 8.97785 \\ 1.79584 & 5.41163 & 8.97650 \\ 8.60609 & 12.23501 & 15.85428 \\ 1.79810 & 12.24058 & 15.85743 \\ 8.61694 & 5.47208 & 22.94375 \\ 1.83035 & 5.38629 & 22.83957 \\ 10.16623 & 10.81510 & 1.26312 \\ 3.34385 & 10.81472 & 1.26317 \\ 10.16105 & 3.98510 & 8.28230 \\ 3.33951 & 3.98586 & 8.28080 \\ 10.15974 & 10.82356 & 15.15188 \\ 3.33846 & 10.81767 & 15.15005 \\ 10.12833 & 4.04023 & 22.17837 \\ 3.36770 & 3.97433 & 22.10234 \\ 5.04613 & 12.86988 & 4.20742 \\ 11.86856 & 12.86883 & 4.20856 \\ & & \\ & & \end{array}$




\begin{tabular}{ccc}
5.01240 & 6.04994 & 11.15237 \\
11.83528 & 6.04935 & 11.15104 \\
5.01576 & 12.86441 & 18.06018 \\
11.86090 & 12.85975 & 18.06352 \\
4.93421 & 6.00579 & 25.06104 \\
12.00818 & 5.98353 & 25.18858 \\
3.52497 & 12.19337 & 5.66672 \\
10.34685 & 12.19215 & 5.66720 \\
3.47748 & 5.37097 & 12.59530 \\
10.30048 & 5.37101 & 12.59423 \\
3.49350 & 12.18370 & 19.51613 \\
10.34289 & 12.16783 & 19.51780 \\
3.45313 & 5.27813 & 26.53947 \\
10.67574 & 5.22667 & 26.80387 \\
4.64446 & 3.00026 & 1.01574 \\
11.46831 & 2.99984 & 1.01649 \\
4.62911 & 9.80392 & 7.99277 \\
11.45308 & 9.80324 & 7.98887 \\
4.61361 & 2.98713 & 14.89383 \\
11.44566 & 2.99341 & 14.90394 \\
4.65978 & 9.77189 & 21.85468 \\
11.55053 & 9.79383 & 21.87792 \\
5.06733 & 0.77187 & 1.32891 \\
11.88900 & 0.77091 & 1.33079 \\
5.03338 & 7.58213 & 8.32881 \\
11.85583 & 7.58155 & 8.32731 \\
5.00712 & 0.76449 & 15.23408 \\
11.84405 & 0.77092 & 15.24449 \\
5.01551 & 7.55945 & 22.30941 \\
11.88541 & 7.56518 & 22.27024 \\
3.53436 & 1.46274 & 46.28492 \\
10.35610 & 1.46295 & 46.28706 \\
3.51419 & 8.26035 & 6.87022 \\
10.33626 & 8.25938 & 6.86886 \\
3.47732 & 1.44697 & 13.78854 \\
10.31056 & 1.45004 & 13.80163 \\
3.56828 & 8.19320 & 20.75875 \\
10.41162 & 8.25777 & 20.76823 \\
7.46636 & 5.48143 & 27.17518 \\
6.41421 & 8.05859 & 27.09532 \\
& & \\
\hline
\end{tabular}


Final state (reaction products) of hydrogen abstraction reaction on PETN (110) surface Method: PBE

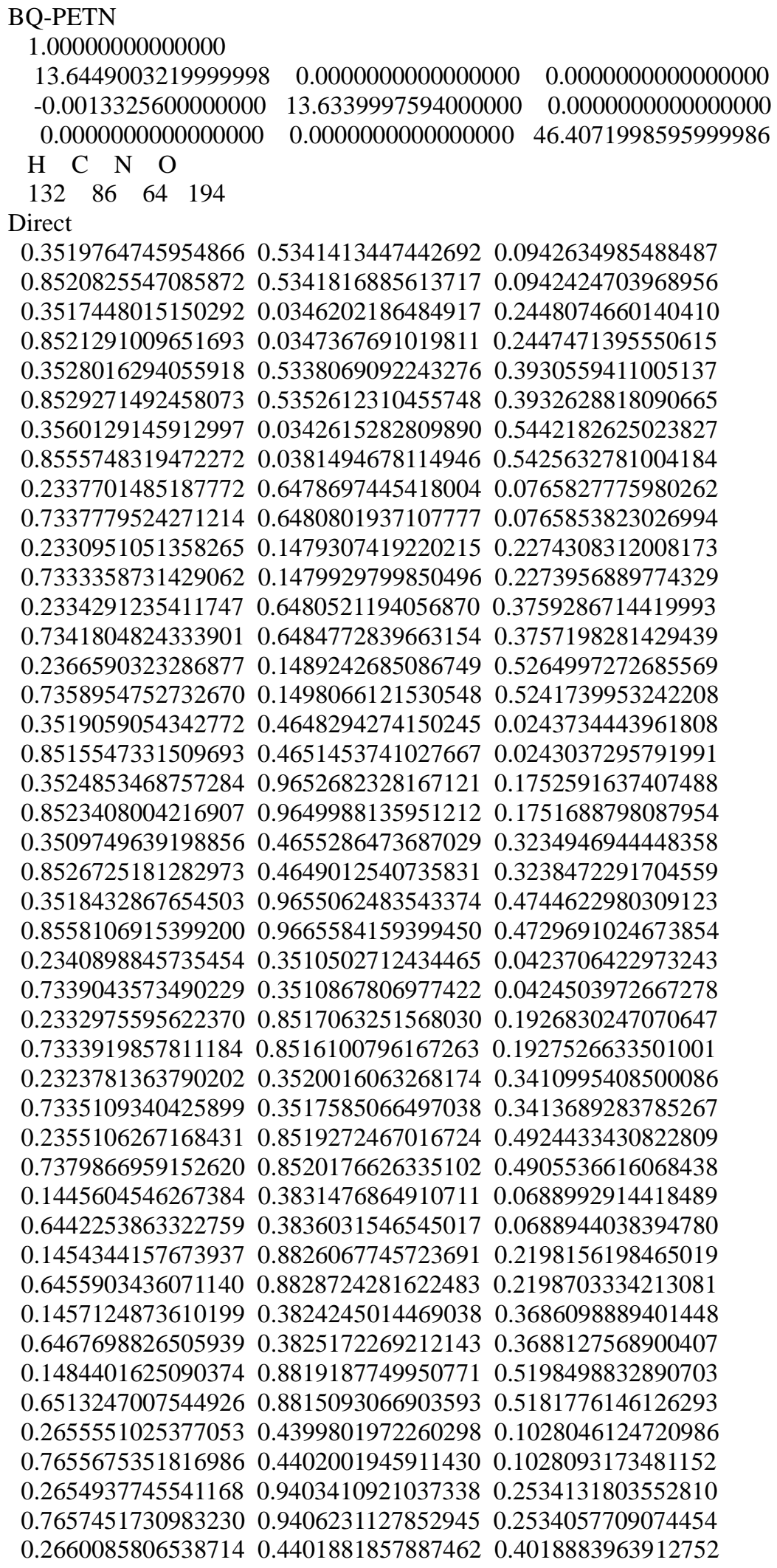


$\begin{array}{llll}0.7666773572599963 & 0.4412261277084394 & 0.4021237904808939\end{array}$ $\begin{array}{llll}0.2701844043842458 & 0.9397812305869419 & 0.5530843444877296\end{array}$ $\begin{array}{llll}0.7708198240339083 & 0.9428949604283516 & 0.5515570276645615\end{array}$ $\begin{array}{llll}0.1453725975498722 & 0.6166747389798648 & 0.0496137011261924\end{array}$ $\begin{array}{llll}0.6452126867735501 & 0.6168029102530882 & 0.0496700982020307\end{array}$ $\begin{array}{llll}0.1464849483581542 & 0.1179277247268240 & 0.1998646501057244\end{array}$ $\begin{array}{llll}0.6466714707738785 & 0.1179945631512827 & 0.1998550634016519\end{array}$ $\begin{array}{llll}0.1454232101352249 & 0.6177981751812545 & 0.3487857601436318\end{array}$ $\begin{array}{llll}0.6466560376819199 & 0.6175816568631851 & 0.3484812886620067\end{array}$ $\begin{array}{llll}0.1450925832423317 & 0.1156944050855546 & 0.5007260004244928\end{array}$ $\begin{array}{lllll}0.6460347629902236 & 0.1145472753616131 & 0.4981138649645891\end{array}$ $\begin{array}{lllll}0.2659628229507854 & 0.5596321837311272 & 0.0159974112603538\end{array}$ $\begin{array}{llll}0.7656674342435654 & 0.5601535896412778 & 0.0160407887532645\end{array}$ $\begin{array}{llll}0.2659194200357332 & 0.0590605639150334 & 0.1664441327352140\end{array}$ $\begin{array}{llll}0.7660657372436100 & 0.0590532265485203 & 0.1663970550641488\end{array}$ $\begin{array}{llll}0.2646607592763614 & 0.5599716302221839 & 0.3150489638831871\end{array}$ $\begin{array}{llll}0.7662409796535828 & 0.5588444082401128 & 0.3150384984386633\end{array}$ $\begin{array}{llll}0.2649293537207100 & 0.0596196070851246 & 0.4662973966128084\end{array}$ $\begin{array}{llll}0.7690428562956602 & 0.0606049046996648 & 0.4645133871595949\end{array}$ $\begin{array}{llll}0.3952747394418283 & 0.8833922773302001 & 0.0496248751237543\end{array}$ $\begin{array}{llll}0.8955534561081674 & 0.8833754465818693 & 0.0496232015489181\end{array}$ $\begin{array}{llll}0.3963913633957615 & 0.3821601961865574 & 0.1998466842968896\end{array}$ $\begin{array}{lllll}0.8965948867040767 & 0.3820337711892497 & 0.1998397401470634\end{array}$ $\begin{array}{llll}0.3942681164623285 & 0.8819603517697334 & 0.3487881582584455\end{array}$ $\begin{array}{lllll}0.8958764944688533 & 0.8828827248198318 & 0.3485538245091283\end{array}$ $\begin{array}{llll}0.3988080454715520 & 0.3737055873098401 & 0.4994442630878517\end{array}$ $\begin{array}{llll}0.8892237453960490 & 0.3841842854460609 & 0.5026350431269493\end{array}$ $\begin{array}{llll}0.5159040917618890 & 0.9402279342654257 & 0.0160504216704384\end{array}$ $\begin{array}{llll}0.0159850530542761 & 0.9404233417150963 & 0.0160472486657194\end{array}$ $\begin{array}{lllll}0.5159168366660613 & 0.4410692573734884 & 0.1664072138306600\end{array}$ $\begin{array}{llll}0.0159558189141037 & 0.4411254759979207 & 0.1663863023822985\end{array}$ $\begin{array}{lllll}0.5138532730374479 & 0.9395492860022704 & 0.3150827189224331\end{array}$ $\begin{array}{llll}0.0159575318943338 & 0.9411923100031495 & 0.3150811638445243\end{array}$ $\begin{array}{lllll}0.5104052833567518 & 0.4272270975881021 & 0.4616655772476074\end{array}$ $\begin{array}{llll}0.0132425230336266 & 0.4353812264662765 & 0.4672663880094351\end{array}$ $\begin{array}{llll}0.3942804922422611 & 0.1165976995181420 & 0.0689007213167039\end{array}$ $\begin{array}{lllll}0.8943605844256285 & 0.1164607178350447 & 0.0689565802476070\end{array}$ $\begin{array}{llll}0.3954896419176069 & 0.6172904058156002 & 0.2198439232644920\end{array}$ $\begin{array}{llll}0.8954512051959541 & 0.6172603471179712 & 0.2198265697494341\end{array}$ $\begin{array}{llll}0.3946284578350508 & 0.1172665126087085 & 0.3684980625086588\end{array}$ $\begin{array}{llll}0.8966482889315639 & 0.1178279712414783 & 0.3687370025588858\end{array}$ $\begin{array}{lllll}0.4044165351790974 & 0.6153660971282895 & 0.5155956289066737\end{array}$ $\begin{array}{llll}0.8919630487817719 & 0.6094656365916697 & 0.5211046182432479\end{array}$ $\begin{array}{llll}0.5155231541848319 & 0.0600575619243678 & 0.1028491500033538\end{array}$ $\begin{array}{llll}0.0156835546352865 & 0.0599491352330400 & 0.1028549757526813\end{array}$ $\begin{array}{llll}0.5154861855231725 & 0.5595800010138915 & 0.2534025859238199\end{array}$ $\begin{array}{lllll}0.0155667926348663 & 0.5595988334023154 & 0.2533852659971771\end{array}$ $\begin{array}{llll}0.5152682150100499 & 0.0598507846997722 & 0.4018882484751381\end{array}$ $\begin{array}{llll}0.0161989014853729 & 0.0590908166626267 & 0.4021597894390438\end{array}$ $\begin{array}{llll}0.4215353751816998 & 0.5356462280781319 & 0.5854859364651243\end{array}$ $\begin{array}{llll}0.0199298306465262 & 0.5588533817143455 & 0.5536435467780378\end{array}$ $\begin{array}{lllll}0.6018081874153576 & 0.9658252689875527 & 0.0942992729902533\end{array}$ $\begin{array}{llll}0.1021773121769137 & 0.9659184634163748 & 0.0942966634253903\end{array}$ $\begin{array}{lllll}0.6017626676739463 & 0.4653267567040754 & 0.2447961142186169\end{array}$ $\begin{array}{llll}0.1017899434321818 & 0.4653370144361476 & 0.2447760489453672\end{array}$ $\begin{array}{llll}0.6010615847593429 & 0.9652123871480299 & 0.3932150746504403\end{array}$ 
$\begin{array}{llll}0.1024599006390990 & 0.9650091840569461 & 0.3933686347269932\end{array}$ $\begin{array}{llll}0.5705118753533358 & 0.4974778192246683 & 0.5495084891728543\end{array}$ $\begin{array}{llll}0.1043045689663454 & 0.4628707599892397 & 0.5452258855775786\end{array}$ $\begin{array}{llll}0.4837798390780503 & 0.8521417149673777 & 0.0765624343349789\end{array}$ $\begin{array}{llll}0.9839425981854196 & 0.8521108773904116 & 0.0765840599322460\end{array}$ $\begin{array}{llll}0.4831470359503738 & 0.3519866980566413 & 0.2273532300866625\end{array}$ $\begin{array}{llll}0.9832364625971942 & 0.3520630970797243 & 0.2273976847444866\end{array}$ $\begin{array}{llll}0.4819715804153817 & 0.8517342078129199 & 0.3760258722302960\end{array}$ $\begin{array}{lllll}0.9836915247352866 & 0.8514838281627911 & 0.3756513505801481\end{array}$ $\begin{array}{lllll}0.4902089757971814 & 0.3525257596937337 & 0.5264142503071236\end{array}$ $\begin{array}{llll}0.9873389578632021 & 0.3452336857992710 & 0.5255439848600393\end{array}$ $\begin{array}{llll}0.6017611980171299 & 0.0351908270684083 & 0.0243846201208206\end{array}$ $\begin{array}{llll}0.1018444810090277 & 0.0353850496266841 & 0.0243918020833255\end{array}$ $\begin{array}{llll}0.6024578950567904 & 0.5348717824684915 & 0.1752316769072751\end{array}$ $\begin{array}{llll}0.1024832254054422 & 0.5349218913775015 & 0.1752137645598054\end{array}$ $\begin{array}{llll}0.6000299593476655 & 0.0340942669237410 & 0.3235540621363837\end{array}$ $\begin{array}{llll}0.1024218031469030 & 0.0350989841550401 & 0.3238829106564012\end{array}$ $\begin{array}{lllll}0.5985390258369283 & 0.5226253254487314 & 0.4680135437832614\end{array}$ $\begin{array}{llll}0.0978314341628614 & 0.5322089893272959 & 0.4747582337007317\end{array}$ $\begin{array}{llll}0.4840137493561923 & 0.1491237792970712 & 0.0424679987046055\end{array}$ $\begin{array}{llll}0.9840372156196644 & 0.1492436556636163 & 0.0425397130118999\end{array}$ $\begin{array}{llll}0.4833223136446869 & 0.6483282713828142 & 0.1927124440003737\end{array}$ $\begin{array}{llll}0.9832597844981564 & 0.6483859863632125 & 0.1926945571835492\end{array}$ $\begin{array}{llll}0.4817988146182927 & 0.1476663406731528 & 0.3411274435613064\end{array}$ $\begin{array}{llll}0.9833868795605244 & 0.1481560313972993 & 0.3412440024471992\end{array}$ $\begin{array}{llll}0.4887051837952848 & 0.6408947062300729 & 0.4869885110687543\end{array}$ $\begin{array}{llll}0.9806760773015754 & 0.6460870774552669 & 0.4947087306181910\end{array}$ $\begin{array}{llll}0.4497010470585597 & 0.6760166951469939 & 0.6508046395659663\end{array}$ $\begin{array}{llll}0.5666062921849575 & 0.6099562337263418 & 0.6874325005511827\end{array}$ $\begin{array}{lllll}0.6601860204555761 & 0.4569055870099605 & 0.6784080657170157\end{array}$ $\begin{array}{llll}0.6389982808652405 & 0.3641905532025750 & 0.6322824718647938\end{array}$ $\begin{array}{llll}0.1873454348834907 & 0.4103943490270386 & 0.0505122862706172\end{array}$ $\begin{array}{llll}0.6872158765531928 & 0.4106001712582476 & 0.0505151475952850\end{array}$ $\begin{array}{llll}0.1869443039874583 & 0.9105915179727088 & 0.2012767124308134\end{array}$ $\begin{array}{llll}0.6871163899701613 & 0.9106286724308219 & 0.2013062348643967\end{array}$ $\begin{array}{llll}0.1863006486204259 & 0.4106996115559917 & 0.3499313871701447\end{array}$ $\begin{array}{lllll}0.6874046497952563 & 0.4105818314355825 & 0.3501158217968678\end{array}$ $\begin{array}{lllll}0.1891078222552894 & 0.9104679363760207 & 0.5012301288102151\end{array}$ $\begin{array}{llll}0.6915925462064550 & 0.9102783957298723 & 0.4994924181744209\end{array}$ $\begin{array}{llll}0.1873106616880846 & 0.5888612568516705 & 0.0680956094747161\end{array}$ $\begin{array}{lllll}0.6872645352762675 & 0.5890397787493866 & 0.0681375358329536\end{array}$ $\begin{array}{llll}0.1869204687247588 & 0.0894101118339114 & 0.2185384016231523\end{array}$ $\begin{array}{lllll}0.6871721619049060 & 0.0894463491239107 & 0.2185121367578835\end{array}$ $\begin{array}{llll}0.1868317297344658 & 0.5894084832751313 & 0.3672920581851347\end{array}$ $\begin{array}{llll}0.6877404441338321 & 0.5896696625349180 & 0.3671127279036444\end{array}$ $\begin{array}{llll}0.1894547528731536 & 0.0891297716891224 & 0.5188745949579694\end{array}$ $\begin{array}{llll}0.6898604250037548 & 0.0891624034637026 & 0.5165157132503159\end{array}$ $\begin{array}{lllll}0.3122782292248548 & 0.4703009028634727 & 0.0857324920066334\end{array}$ $\begin{array}{lllll}0.8122735603279579 & 0.4704123589423587 & 0.0857163376386712\end{array}$ $\begin{array}{llll}0.3119684925902602 & 0.9708090131077779 & 0.2363147135343257\end{array}$ $\begin{array}{llll}0.8122629355635697 & 0.9709201707941720 & 0.2362893264235559\end{array}$ $\begin{array}{lllll}0.3121870420434631 & 0.4703531000429937 & 0.3846707590577468\end{array}$ $\begin{array}{llll}0.8129678585811543 & 0.4713010643523051 & 0.3849292563045963\end{array}$ $\begin{array}{llll}0.3158788255848324 & 0.9704380845236709 & 0.5358113733937564\end{array}$ $\begin{array}{llll}0.8169029340504398 & 0.9732208037425597 & 0.5343282004478032\end{array}$ $\begin{array}{llll}0.2500836454795360 & 0.4995965167680831 & 0.0593456790496834\end{array}$ 
$\begin{array}{llll}0.7500133610974753 & 0.49978886651695598 & 0.0593516869459497\end{array}$ $\begin{array}{lllll}0.2496496065275707 & 0.9999680671418156 & 0.2099508539766904\end{array}$ $\begin{array}{lllll}0.7498995040430387 & 0.0000020778697623 & 0.2099204489563635\end{array}$ $\begin{array}{lllll}0.2492267224296950 & 0.5000136788154710 & 0.3584857548802548\end{array}$ $\begin{array}{lllll}0.7502781640212249 & 0.5000537496250145 & 0.3585935449703654\end{array}$ $\begin{array}{lllll}0.2518491238829906 & 0.9999460262492911 & 0.5098115667240557\end{array}$ $\begin{array}{lllll}0.7536858695564566 & 0.0004485093874574 & 0.5079414070459368\end{array}$ $\begin{array}{lllll}0.3126546616008540 & 0.5288372345923078 & 0.0330083111260270\end{array}$ $\begin{array}{lllll}0.8124534141874089 & 0.5291416787777600 & 0.0330018597928259\end{array}$ $\begin{array}{lllll}0.3122985020624569 & 0.0290150395947335 & 0.1836293837286468\end{array}$ $\begin{array}{lllll}0.8124462823415870 & 0.0288957714507808 & 0.1835641328433071\end{array}$ $\begin{array}{lllll}0.3112993278590191 & 0.5292676360757727 & 0.3320686776385566\end{array}$ $\begin{array}{lllll}0.8126658357342887 & 0.5287696897918165 & 0.3322036180869943\end{array}$ $\begin{array}{lllll}0.3125812631688099 & 0.0293458970870414 & 0.4831466883505872\end{array}$ $\begin{array}{lllll}0.8157040011552963 & 0.0300189874312315 & 0.4815540240236368\end{array}$ $\begin{array}{lllll}0.4372477915197372 & 0.9111417943658632 & 0.0681104704146109\end{array}$ $\begin{array}{lllll}0.9374812370240682 & 0.9111412663510114 & 0.0681162325950731\end{array}$ $\begin{array}{lllll}0.4369686584187361 & 0.4105731305831988 & 0.2185089411423096\end{array}$ $\begin{array}{lllll}0.9370440247199766 & 0.4105732057796849 & 0.2185099790563104\end{array}$ $\begin{array}{lllll}0.4355538262671825 & 0.9103744116535134 & 0.3673142109857145\end{array}$ $\begin{array}{lllll}0.9372949880716530 & 0.9105116599087001 & 0.3671560753762897\end{array}$ $\begin{array}{lllll}0.4424785775702348 & 0.4080605569526095 & 0.5165686130154609\end{array}$ $\begin{array}{lllll}0.9391696120091367 & 0.4068796693389164 & 0.5199756984311636\end{array}$ $\begin{array}{lllll}0.4373091642118743 & 0.0896128044747965 & 0.0505326423614896\end{array}$ $\begin{array}{lllll}0.9374356600263108 & 0.0896191177014030 & 0.0505799301216264\end{array}$ $\begin{array}{lllll}0.4369709741075886 & 0.5894053039241408 & 0.2012897620056823\end{array}$ $\begin{array}{lllll}0.9369622214821198 & 0.5894072851482053 & 0.2012734538163762\end{array}$ $\begin{array}{llllll}0.4355229666814243 & 0.0889963369901154 & 0.3498832375201339\end{array}$ $\begin{array}{lllll}0.9372309243569068 & 0.0894974734634586 & 0.3500690514530415\end{array}$ $\begin{array}{llllll}0.4415802985355269 & 0.5845438751507548 & 0.4966521898861241\end{array}$ $\begin{array}{lllll}0.9355731106054607 & 0.5849557018058716 & 0.5025275545178920\end{array}$ $\begin{array}{llllll}0.5621913610568191 & 0.0297214032851584 & 0.0857689355038251\end{array}$ $\begin{array}{lllll}0.0624282533184970 & 0.0297327270496536 & 0.0857789015473665\end{array}$ $\begin{array}{lllll}0.5620065088399221 & 0.5291550695555050 & 0.2363085550821499\end{array}$ $\begin{array}{lllll}0.0620650959168570 & 0.5291804428888677 & 0.2362877563977297\end{array}$ $\begin{array}{lllll}0.5612179504973086 & 0.0289941024717420 & 0.3847236569332476\end{array}$ $\begin{array}{lllll}0.0625432841750801 & 0.0289177130665899 & 0.3849866612948961\end{array}$ $\begin{array}{lllll}0.5780682422570773 & 0.5294778153033228 & 0.5275040542546493\end{array}$ $\begin{array}{llllll}0.0651322192773478 & 0.5268002100088671 & 0.5365043362170743\end{array}$ $\begin{array}{llll}0.5000254570009706 & 0.0004318374887162 & 0.0593729545583658\end{array}$ $\begin{array}{lllll}0.0002419123234029 & 0.0004621511587464 & 0.0593900200573011\end{array}$ $\begin{array}{lllll}0.4996988598190339 & 0.5000345428489620 & 0.2099357770556003\end{array}$ $\begin{array}{lllll}0.9997306204353525 & 0.5000624016895621 & 0.2099181741502929\end{array}$ $\begin{array}{lllll}0.4982072253024049 & 0.9996226817091357 & 0.3585111091255220\end{array}$ $\begin{array}{lllll}0.9999836355542649 & 0.0000418794488793 & 0.3586239552043685\end{array}$ $\begin{array}{lllll}0.5033298689498951 & 0.4937802494911150 & 0.5046894421439805\end{array}$ $\begin{array}{llll}0.0004203029385452 & 0.4965643446242183 & 0.5106934363566399\end{array}$ $\begin{array}{lllll}0.5626208277352502 & 0.9711437106561677 & 0.0330382467768395\end{array}$ $\begin{array}{lllll}0.0627344373631641 & 0.9713057865017011 & 0.0330333910925154\end{array}$ $\begin{array}{lllll}0.5623209250550140 & 0.4710763010837277 & 0.1835944115645166\end{array}$ $\begin{array}{llllll}0.0623304126229382 & 0.4711324113906845 & 0.1835795121730753\end{array}$ $\begin{array}{lllll}0.5603953794052693 & 0.9703024649195190 & 0.3321177862258181\end{array}$ $\begin{array}{lllll}0.0624112561836426 & 0.9712328655351539 & 0.3322472156117113\end{array}$ $\begin{array}{lllll}0.5595546754471457 & 0.4602599497368132 & 0.4776535973057254\end{array}$ $\begin{array}{lllll}0.0606522820005586 & 0.4677408628255537 & 0.4838407582178624\end{array}$ $\begin{array}{lllll}0.5316348730697052 & 0.4661754639369912 & 0.6150205031502416\end{array}$ 
$\begin{array}{llll}0.4775178021496921 & 0.5571791996418666 & 0.6212512207512684\end{array}$ $\begin{array}{llll}0.4904096985274931 & 0.6084847668271858 & 0.6469603878838156\end{array}$ $\begin{array}{llll}0.5559964924942707 & 0.5711000864156991 & 0.6671196526342935\end{array}$ $\begin{array}{llll}0.6094844697152464 & 0.4830781638253302 & 0.6619447649519206\end{array}$ $\begin{array}{lllll}0.5984089028946089 & 0.4313934024774021 & 0.6366926157297187\end{array}$ $\begin{array}{llll}0.0678431149155306 & 0.3631392328950618 & 0.0142291102347534\end{array}$ $\begin{array}{lllll}0.5681729572668078 & 0.3631957591287287 & 0.0141157802870718\end{array}$ $\begin{array}{llll}0.0675542959952569 & 0.8639196255102972 & 0.1651793349501700\end{array}$ $\begin{array}{llll}0.5676793249126901 & 0.8637774607454450 & 0.1652426474942851\end{array}$ $\begin{array}{llll}0.0660763341796824 & 0.3639090199650180 & 0.3141330053113515\end{array}$ $\begin{array}{llll}0.5667297322192917 & 0.3635370735534316 & 0.3144458049496000\end{array}$ $\begin{array}{llll}0.0694110705184020 & 0.8633996104821644 & 0.4651730267855387\end{array}$ $\begin{array}{llll}0.5703636696288544 & 0.8631047450081888 & 0.4639249121905378\end{array}$ $\begin{array}{llll}0.0685580863411407 & 0.6358637577895597 & 0.1043671419537212\end{array}$ $\begin{array}{llll}0.5685713768356622 & 0.6360808138176944 & 0.1044164160444274\end{array}$ $\begin{array}{llll}0.0661190822958025 & 0.1361321707727461 & 0.2541759841317553\end{array}$ $\begin{array}{llll}0.5663510530141092 & 0.1361430289545573 & 0.2541554789321214\end{array}$ $\begin{array}{lllll}0.0674803773520244 & 0.6361233346892433 & 0.4033861614680747\end{array}$ $\begin{array}{llll}0.5672263769338916 & 0.6371949125462746 & 0.4027403077087373\end{array}$ $\begin{array}{lllll}0.0742443627283359 & 0.1380052005069236 & 0.5561514858469186\end{array}$ $\begin{array}{llll}0.5721901785458804 & 0.1371780388263777 & 0.5532274568819076\end{array}$ $\begin{array}{lllll}0.4337883569410398 & 0.3510370573262644 & 0.1001855755241211\end{array}$ $\begin{array}{llll}0.9338461354093737 & 0.3510975905528496 & 0.1001258782144467\end{array}$ $\begin{array}{llll}0.4332706982107059 & 0.8508558119266272 & 0.2504807967200505\end{array}$ $\begin{array}{lllll}0.9336837558516811 & 0.8511051438839798 & 0.2505029797243334\end{array}$ $\begin{array}{llll}0.4312641153567752 & 0.3475541632615821 & 0.3983726114275599\end{array}$ $\begin{array}{lllll}0.9345735884193603 & 0.3515926005781491 & 0.3991514343785757\end{array}$ $\begin{array}{llll}0.4381857701318380 & 0.8505602784853453 & 0.5496323139656013\end{array}$ $\begin{array}{llll}0.9441977177900452 & 0.8609773545031566 & 0.5491532150660899\end{array}$ $\begin{array}{llll}0.4325429660099178 & 0.6497771717243439 & 0.0183585381463281\end{array}$ $\begin{array}{llll}0.9326239120734164 & 0.6498893711913520 & 0.0183935670137400\end{array}$ $\begin{array}{llll}0.4336420709097671 & 0.1489924882432819 & 0.1694582384315111\end{array}$ $\begin{array}{llll}0.9338484119214575 & 0.1486455710136984 & 0.1693041218422036\end{array}$ $\begin{array}{llll}0.4332634118186706 & 0.6485658136594390 & 0.3179035432786118\end{array}$ $\begin{array}{llll}0.9336967614270029 & 0.6488136683718714 & 0.3179233436238541\end{array}$ $\begin{array}{llll}0.4340554721473963 & 0.1474516541504899 & 0.4680949190705405\end{array}$ $\begin{array}{llll}0.9375778542554509 & 0.1499350380269548 & 0.4677099848039531\end{array}$ $\begin{array}{lllll}0.6837565571948264 & 0.1490727781161077 & 0.1002003801866339\end{array}$ $\begin{array}{llll}0.1839966070671032 & 0.1490155601705054 & 0.1002216673759750\end{array}$ $\begin{array}{llll}0.6835076932460634 & 0.6489481058203982 & 0.2505046892269789\end{array}$ $\begin{array}{llll}0.1834560771831422 & 0.6490063211328509 & 0.2505013197611712\end{array}$ $\begin{array}{llll}0.6842011725452718 & 0.1479978321743586 & 0.3983557864232392\end{array}$ $\begin{array}{llll}0.1841904383111453 & 0.1486647796994330 & 0.3991835959672991\end{array}$ $\begin{array}{lllll}0.7092061969512518 & 0.6864529039192117 & 0.5721437487512276\end{array}$ $\begin{array}{llll}0.1841844714486849 & 0.6504855699892010 & 0.5500177295708228\end{array}$ $\begin{array}{llll}0.6825708222463966 & 0.8501792676984842 & 0.0184250290822323\end{array}$ $\begin{array}{llll}0.1827011964509860 & 0.8505467497356225 & 0.0183516625305568\end{array}$ $\begin{array}{llll}0.6836864690182403 & 0.3511286427809088 & 0.1693942830495550\end{array}$ $\begin{array}{lllll}0.1835809971427476 & 0.3511387925368231 & 0.1693702184882090\end{array}$ $\begin{array}{llll}0.6827694487256090 & 0.8514392719613624 & 0.3179891997693562\end{array}$ $\begin{array}{llll}0.1833580479155917 & 0.8511325835725908 & 0.3179390537969712\end{array}$ $\begin{array}{lllll}0.6850629258746562 & 0.3438212038363335 & 0.4644119688654949\end{array}$ $\begin{array}{lllll}0.1839487943740766 & 0.3508903161102267 & 0.4689051279017049\end{array}$ $\begin{array}{llll}0.3185766894665195 & 0.8639992599491557 & 0.1044008349922180\end{array}$ $\begin{array}{llll}0.8186581203002428 & 0.8640713171078621 & 0.1043600727620700\end{array}$ $\begin{array}{llll}0.3161925272627613 & 0.3638217487663073 & 0.2541661351650126\end{array}$ 
$\begin{array}{llll}0.8162317991938183 & 0.3637983858364141 & 0.2541558490299611\end{array}$ $\begin{array}{llll}0.3163345054413868 & 0.8638271788469848 & 0.4034674196039601\end{array}$ $\begin{array}{llll}0.8181090319645726 & 0.8628663703924428 & 0.4032051717998262\end{array}$ $\begin{array}{llll}0.3263282737498044 & 0.3724258493208499 & 0.5546855392950530\end{array}$ $\begin{array}{llll}0.8316390888833486 & 0.3549370841943321 & 0.5588069885293563\end{array}$ $\begin{array}{llll}0.3181555898410522 & 0.1369396340122818 & 0.0141681218344315\end{array}$ $\begin{array}{llll}0.8184269111916072 & 0.1369157544508327 & 0.0141846590802534\end{array}$ $\begin{array}{llll}0.3175728310366026 & 0.6362831126778266 & 0.1652231101493133\end{array}$ $\begin{array}{llll}0.8175266078884221 & 0.6361017037803935 & 0.1651926203945196\end{array}$ $\begin{array}{llll}0.3153872629745550 & 0.1359505889424104 & 0.3141164130650134\end{array}$ $\begin{array}{llll}0.8165992794643765 & 0.1362662931553733 & 0.3143682780472721\end{array}$ $\begin{array}{llll}0.3146581059287432 & 0.6324490716160011 & 0.4631337336553862\end{array}$ $\begin{array}{llll}0.8174318211282734 & 0.6314101295342082 & 0.4660218495615077\end{array}$ $\begin{array}{lllll}0.0892685742130683 & 0.2800085567850718 & 0.0214357901997627\end{array}$ $\begin{array}{llll}0.5895640712860098 & 0.2800878538541157 & 0.0213545125798727\end{array}$ $\begin{array}{llll}0.0891706210452528 & 0.7806747586631049 & 0.1721255945578241\end{array}$ $\begin{array}{llll}0.5893681553596536 & 0.7805632442607139 & 0.1722066985343016\end{array}$ $\begin{array}{llll}0.0886376404635289 & 0.2807084949471339 & 0.3208427607743103\end{array}$ $\begin{array}{llll}0.5890884561378626 & 0.2803444644640590 & 0.3212246035384257\end{array}$ $\begin{array}{llll}0.0921084378463491 & 0.7802017775638145 & 0.4718102697882062\end{array}$ $\begin{array}{llll}0.5928608764287719 & 0.7799891527822325 & 0.4707164055960885\end{array}$ $\begin{array}{llll}0.1212198766664620 & 0.4433172060346180 & 0.0280735803451677\end{array}$ $\begin{array}{llll}0.6213601363469807 & 0.4434418485543057 & 0.0279963216527670\end{array}$ $\begin{array}{llll}0.1190196530020024 & 0.9436369725607835 & 0.1793010803717596\end{array}$ $\begin{array}{lllll}0.6192059665421991 & 0.9435667452798566 & 0.1793145756583930\end{array}$ $\begin{array}{llll}0.1173291566546863 & 0.4437549526147124 & 0.3282519681271621\end{array}$ $\begin{array}{lllll}0.6185125540511363 & 0.4434220332194874 & 0.3283948409029537\end{array}$ $\begin{array}{lllll}0.1202854019633752 & 0.9433665596306087 & 0.4794924761850472\end{array}$ $\begin{array}{lllll}0.6221395202706009 & 0.9430356751715137 & 0.4778898447602223\end{array}$ $\begin{array}{llll}0.0081339967354805 & 0.3930990984881634 & 0.9969812105969696\end{array}$ $\begin{array}{llll}0.5086059156938613 & 0.3930809663911171 & 0.9968136246635680\end{array}$ $\begin{array}{llll}0.0078438815658680 & 0.8938585937845646 & 0.1478140468814587\end{array}$ $\begin{array}{lllll}0.5078599275151964 & 0.8936413824558472 & 0.1478987152357757\end{array}$ $\begin{array}{llll}0.0055237832868195 & 0.3937270586943881 & 0.2969991973618892\end{array}$ $\begin{array}{llll}0.5060125983714592 & 0.3933676096425385 & 0.2973671937561341\end{array}$ $\begin{array}{llll}0.0090428853599275 & 0.8933686194921859 & 0.4480133795994118\end{array}$ $\begin{array}{llll}0.5096057856529471 & 0.8929192485743402 & 0.4468604606136014\end{array}$ $\begin{array}{llll}0.0902347026445466 & 0.7190093429848602 & 0.0973402836898150\end{array}$ $\begin{array}{llll}0.5900203867477569 & 0.7192261846884388 & 0.0973237266917173\end{array}$ $\begin{array}{llll}0.0887745143519673 & 0.2193392372154740 & 0.2474988785676767\end{array}$ $\begin{array}{llll}0.5888917108610362 & 0.2193753872710559 & 0.2474675586890120\end{array}$ $\begin{array}{llll}0.0891935674940903 & 0.7193029765095608 & 0.3963972957482555\end{array}$ $\begin{array}{llll}0.5893791706028939 & 0.7202665934002358 & 0.3957755968718071\end{array}$ $\begin{array}{lllll}0.0953126618220308 & 0.2207642782832814 & 0.5484782761531365\end{array}$ $\begin{array}{llll}0.5931072925505210 & 0.2198772299445675 & 0.5456611081324841\end{array}$ $\begin{array}{llll}0.1198894777133224 & 0.5558791993483256 & 0.0901917259659909\end{array}$ $\begin{array}{llll}0.6200019947339271 & 0.5560978013073885 & 0.0902803500040531\end{array}$ $\begin{array}{llll}0.1177550832855609 & 0.0563037896566851 & 0.2401490834733692\end{array}$ $\begin{array}{llll}0.6180686122955673 & 0.0563326697316880 & 0.2401385745039165\end{array}$ $\begin{array}{lllll}0.1189209021304751 & 0.5562856380941065 & 0.3892290380146579\end{array}$ $\begin{array}{lllll}0.6193176293510066 & 0.5571378186047207 & 0.3890089582137105\end{array}$ $\begin{array}{llll}0.1252604055790458 & 0.0569614505231658 & 0.5418801028352314\end{array}$ $\begin{array}{llll}0.6253242120801177 & 0.0563739475946825 & 0.5393027500792053\end{array}$ $\begin{array}{lllll}0.0090229980811237 & 0.6060452445689121 & 0.1217863348787339\end{array}$ $\begin{array}{llll}0.5091956610177081 & 0.6062627450239741 & 0.1218830143075891\end{array}$ $\begin{array}{llll}0.0052148132513443 & 0.1062383235554043 & 0.2711838929615147\end{array}$ 
$\begin{array}{llll}0.5055072312318378 & 0.1061980221927471 & 0.2711742064878162\end{array}$ $\begin{array}{llll}0.0078148352282636 & 0.6062321998264746 & 0.4207464006871250\end{array}$ $\begin{array}{llll}0.5065051765776302 & 0.6076522024393760 & 0.4198638981833793\end{array}$ $\begin{array}{llll}0.0166248067521765 & 0.1090133462544813 & 0.5741122788173412\end{array}$ $\begin{array}{llll}0.5135442618667312 & 0.1073191787510411 & 0.5707914554533342\end{array}$ $\begin{array}{llll}0.4105882315890043 & 0.3751824842181349 & 0.1244529569581413\end{array}$ $\begin{array}{llll}0.9107865960317862 & 0.3752353683745790 & 0.1244042746414518\end{array}$ $\begin{array}{llll}0.4095295077654599 & 0.8736426075948345 & 0.2748243926882452\end{array}$ $\begin{array}{llll}0.9103823425373763 & 0.8743915140049120 & 0.2748427951354657\end{array}$ $\begin{array}{llll}0.4077854387795319 & 0.3695860795298108 & 0.4228013003786761\end{array}$ $\begin{array}{llll}0.9118287155325885 & 0.3753329666240262 & 0.4234827625959297\end{array}$ $\begin{array}{llll}0.4166650629946963 & 0.8746892457356993 & 0.5739830888316626\end{array}$ $\begin{array}{llll}0.9256928738827888 & 0.8912676399265045 & 0.5731150741870243\end{array}$ $\begin{array}{llll}0.3821645258074681 & 0.3970917501524800 & 0.0763436454504998\end{array}$ $\begin{array}{llll}0.8820610740425867 & 0.3971083559403881 & 0.0763102690370546\end{array}$ $\begin{array}{llll}0.3818515693950345 & 0.8977144858114949 & 0.2268021002310115\end{array}$ $\begin{array}{llll}0.8820074976455963 & 0.8976557058968423 & 0.2268113637369740\end{array}$ $\begin{array}{llll}0.3810960817439338 & 0.3967220716197939 & 0.3748975479135291\end{array}$ $\begin{array}{llll}0.8825171404172772 & 0.3978929286294591 & 0.3754499770501358\end{array}$ $\begin{array}{llll}0.3849557139227326 & 0.8973907705997907 & 0.5259688988140707\end{array}$ $\begin{array}{llll}0.8875203634392029 & 0.9004796735448288 & 0.5251884683189187\end{array}$ $\begin{array}{llll}0.4952020668189007 & 0.2930115968028725 & 0.0917871621426502\end{array}$ $\begin{array}{llll}0.9952183294338802 & 0.2930746835143709 & 0.0916975038017555\end{array}$ $\begin{array}{llll}0.4951078966772443 & 0.7935258469298945 & 0.2419357698512215\end{array}$ $\begin{array}{llll}0.9952710426885725 & 0.7935003118614370 & 0.2419567251431944\end{array}$ $\begin{array}{llll}0.4919315733592940 & 0.2893537061674826 & 0.3896218027745967\end{array}$ $\begin{array}{llll}0.9958263774624566 & 0.2936994476885545 & 0.3905666501300776\end{array}$ $\begin{array}{llll}0.4986593591347260 & 0.7923259310464540 & 0.5407714627279394\end{array}$ $\begin{array}{llll}0.0041607955464349 & 0.8008624187860615 & 0.5410005595575460\end{array}$ $\begin{array}{llll}0.4077900001402260 & 0.6274245424992876 & 0.9941351917442804\end{array}$ $\begin{array}{llll}0.9078637858907060 & 0.6276311225337899 & 0.9941621355268546\end{array}$ $\begin{array}{lllll}0.4109886955917533 & 0.1251940956720154 & 0.1451087444830002\end{array}$ $\begin{array}{llll}0.9107549911121960 & 0.1250764868396185 & 0.1449693343623015\end{array}$ $\begin{array}{llll}0.4101322178536065 & 0.6250779162598508 & 0.2935622499629265\end{array}$ $\begin{array}{llll}0.9103556463149601 & 0.6253819039330006 & 0.2935934398661363\end{array}$ $\begin{array}{llll}0.4101490952074483 & 0.1224043614700770 & 0.4439614484070894\end{array}$ $\begin{array}{llll}0.9155479410508299 & 0.1262401463132790 & 0.4433099656231398\end{array}$ $\begin{array}{llll}0.3828570927978975 & 0.6016064906759476 & 0.0423480445331669\end{array}$ $\begin{array}{llll}0.8828530634600603 & 0.6017218640901091 & 0.0423647264102437\end{array}$ $\begin{array}{lllll}0.3817115234070914 & 0.1026098127877371 & 0.1931278777348861\end{array}$ $\begin{array}{llll}0.8821662313009709 & 0.1022615518809666 & 0.1930114941508845\end{array}$ $\begin{array}{llll}0.3813777335153034 & 0.6023091607070555 & 0.3415693740117958\end{array}$ $\begin{array}{llll}0.8823059752944703 & 0.6022696369257980 & 0.3416250782340643\end{array}$ $\begin{array}{llll}0.3829202585286799 & 0.1026641716230106 & 0.4921775055067319\end{array}$ $\begin{array}{llll}0.8852474850616339 & 0.1033020669048640 & 0.4911998062705429\end{array}$ $\begin{array}{llll}0.4941946153298571 & 0.7076290864003419 & 0.0268308746357092\end{array}$ $\begin{array}{llll}0.9943305889279649 & 0.7076644597045010 & 0.0268817558930783\end{array}$ $\begin{array}{llll}0.4947948776629897 & 0.2070678346544167 & 0.1780225910130279\end{array}$ $\begin{array}{llll}0.9952711798415986 & 0.2065034626586660 & 0.1778261485339713\end{array}$ $\begin{array}{llll}0.4948532498986302 & 0.7062274220517275 & 0.3264244439863812\end{array}$ $\begin{array}{llll}0.9951655680640061 & 0.7066450535041701 & 0.3264130677190624\end{array}$ $\begin{array}{llll}0.4959118176114404 & 0.2056258734944744 & 0.4761306049948317\end{array}$ $\begin{array}{llll}0.9983974845180148 & 0.2080754548445140 & 0.4764345598865795\end{array}$ $0.6604926327934414 \quad 0.1251602446440554 \quad 0.1244828704064623$ $\begin{array}{llll}0.1608757614612405 & 0.1248780592660330 & 0.1244977716413142\end{array}$ $\begin{array}{llll}0.6601188562922421 & 0.6258004109672172 & 0.2748496456761194\end{array}$ 
$\begin{array}{llll}0.1599384987358493 & 0.6259055974417097 & 0.2748386101281993\end{array}$ $\begin{array}{llll}0.6620386914213561 & 0.1248498638310909 & 0.4227954005757705\end{array}$ $\begin{array}{llll}0.1615828157852234 & 0.1248963295731414 & 0.4235243110559743\end{array}$ $\begin{array}{llll}0.7032389319156702 & 0.6200762635520579 & 0.5893570937863525\end{array}$ $\begin{array}{llll}0.1580589361705018 & 0.6323431543421842 & 0.5744105218804285\end{array}$ $\begin{array}{llll}0.6321017539362924 & 0.1029141942436750 & 0.0763779608073797\end{array}$ $\begin{array}{llll}0.1322561083178738 & 0.1030290331146885 & 0.0763960983498213\end{array}$ $\begin{array}{lllll}0.6318637106739418 & 0.6023230241941474 & 0.2268206004502265\end{array}$ $\begin{array}{lllll}0.1319383029039292 & 0.6023263451678151 & 0.2268052705146887\end{array}$ $\begin{array}{llll}0.6309601232877430 & 0.1017922589105097 & 0.3749186601900604\end{array}$ $\begin{array}{llll}0.1321867903273163 & 0.1022900497289983 & 0.3755048521658066\end{array}$ $\begin{array}{llll}0.6416746301529382 & 0.5891390202350211 & 0.5217830537136749\end{array}$ $\begin{array}{llll}0.1354482769200873 & 0.5983341575714662 & 0.5264667030458624\end{array}$ $\begin{array}{llll}0.7452492941980030 & 0.2069668101117290 & 0.0917670296505726\end{array}$ $\begin{array}{llll}0.2454124296571791 & 0.2070237335751599 & 0.0918023921983238\end{array}$ $\begin{array}{llll}0.7451728019003676 & 0.7064681607448478 & 0.2419510765859809\end{array}$ $\begin{array}{llll}0.2451609925909034 & 0.7065159714574971 & 0.2419688838907295\end{array}$ $\begin{array}{llll}0.7457125354587498 & 0.2052068600043297 & 0.3895298862314287\end{array}$ $\begin{array}{llll}0.2453397622037002 & 0.2066724194130812 & 0.3905838817133401\end{array}$ $\begin{array}{llll}0.7777944835487999 & 0.7269054335513361 & 0.5604639185561046\end{array}$ $\begin{array}{llll}0.2463202349626278 & 0.7066445903796911 & 0.5409623074673899\end{array}$ $\begin{array}{llll}0.3401288294611829 & 0.7808682039592595 & 0.0973260190453914\end{array}$ $\begin{array}{llll}0.8402184076938563 & 0.7809282601075428 & 0.0972974311085891\end{array}$ $\begin{array}{lllll}0.3387729256634620 & 0.2806043810628755 & 0.2474778623540488\end{array}$ $\begin{array}{lllll}0.8388020041837266 & 0.2805862677801217 & 0.2474634389213704\end{array}$ $\begin{array}{llll}0.3387317586154807 & 0.7806058882181833 & 0.3967193429990658\end{array}$ $\begin{array}{llll}0.8401819611598715 & 0.7798037109535420 & 0.3961987166360824\end{array}$ $\begin{array}{llll}0.3430430663704398 & 0.2876126425084362 & 0.5483776194725112\end{array}$ $\begin{array}{llll}0.8425172773650877 & 0.2740538283422692 & 0.5480710652585855\end{array}$ $\begin{array}{llll}0.6577360529077970 & 0.8722947490501028 & 0.9941915957542287\end{array}$ $\begin{array}{llll}0.1576644108086318 & 0.8727013436036412 & 0.9941368306327303\end{array}$ $\begin{array}{llll}0.6610343109343747 & 0.3749138754213331 & 0.1450433752777701\end{array}$ $\begin{array}{llll}0.1607712779091852 & 0.3748446395311822 & 0.1450245264702265\end{array}$ $\begin{array}{llll}0.6597727844843989 & 0.8749562507809590 & 0.2936376942129649\end{array}$ $\begin{array}{llll}0.1599517633755360 & 0.8745313316397642 & 0.2936153636578945\end{array}$ $\begin{array}{llll}0.6629586229801397 & 0.3669173942728666 & 0.4399256445019814\end{array}$ $\begin{array}{lllll}0.1590792724370542 & 0.3742884903001085 & 0.4447189361848829\end{array}$ $\begin{array}{llll}0.6329226493116816 & 0.8985081019112274 & 0.0423952694920497\end{array}$ $\begin{array}{llll}0.1330946215922859 & 0.8986767628170069 & 0.0423538338441302\end{array}$ $\begin{array}{llll}0.6317698640703703 & 0.3974919497286403 & 0.1930694599240573\end{array}$ $\begin{array}{llll}0.1317898058543833 & 0.3975560963147106 & 0.1930515082228048\end{array}$ $\begin{array}{llll}0.6305147585614490 & 0.8973739111379673 & 0.3416401135975289\end{array}$ $\begin{array}{llll}0.1320686613513881 & 0.8977271282666920 & 0.3416547592337520\end{array}$ $\begin{array}{llll}0.6303376407074948 & 0.3884437804891642 & 0.4876254422366102\end{array}$ $\begin{array}{llll}0.1332770835639048 & 0.3966882395849751 & 0.4929134265564075\end{array}$ $\begin{array}{llll}0.7442851164592381 & 0.7924453664821698 & 0.0269206483671397\end{array}$ $\begin{array}{llll}0.2445905349775324 & 0.7929138607240038 & 0.0267995340015094\end{array}$ $\begin{array}{llll}0.7448402477031341 & 0.2930658738872219 & 0.1779577135559172\end{array}$ $\begin{array}{llll}0.2448012259778223 & 0.2931238447273719 & 0.1779206821772242\end{array}$ $\begin{array}{llll}0.7445215053463213 & 0.7939762852354205 & 0.3265210077463566\end{array}$ $\begin{array}{llll}0.2448528901980736 & 0.7933098590365192 & 0.3264153844834390\end{array}$ $\begin{array}{llll}0.7475206440849508 & 0.2877030534446323 & 0.4732309983964301\end{array}$ $\begin{array}{llll}0.2465307330186697 & 0.2936861462399956 & 0.4770681010472425\end{array}$ $\begin{array}{llll}0.3699005649143488 & 0.9440185064595474 & 0.0902412493041524\end{array}$ $\begin{array}{llll}0.8700792159683844 & 0.9440489214022901 & 0.0902243225952208\end{array}$ $\begin{array}{llll}0.3679599841425643 & 0.4436430455808532 & 0.2401670398718575\end{array}$ 
$\begin{array}{llll}0.8679337385078066 & 0.4436423567665185 & 0.2401347922855645\end{array}$ $\begin{array}{lllll}0.3673529763162472 & 0.9436209050123363 & 0.3891846509212586\end{array}$ $\begin{array}{lllll}0.8693078720383668 & 0.9430296296345824 & 0.3891660825092907\end{array}$ $\begin{array}{lllll}0.3775299978838036 & 0.44830176666648836 & 0.5382040105269247\end{array}$ $\begin{array}{llllll}0.8831033794711445 & 0.4365128529939253 & 0.5450096909907920\end{array}$ $\begin{array}{lllll}0.2591720437757810 & 0.8937815560779870 & 0.1218634363839282\end{array}$ $\begin{array}{lllll}0.7591722525197078 & 0.8938792201469082 & 0.1217943805832091\end{array}$ $\begin{array}{lllll}0.2552838562173320 & 0.3937221321428276 & 0.2711720801945061\end{array}$ $\begin{array}{lllll}0.7553791833407237 & 0.3936994437572574 & 0.2711749621970624\end{array}$ $\begin{array}{lllll}0.2561997066995623 & 0.8937297583190793 & 0.4206988337954305\end{array}$ $\begin{array}{lllll}0.7581756609689637 & 0.8923782372762079 & 0.4205575986944229\end{array}$ $\begin{array}{lllll}0.2724348569230636 & 0.4078257447743567 & 0.5727223255696690\end{array}$ $\begin{array}{llllll}0.7845004163065623 & 0.3812754613593397 & 0.5795133814138966\end{array}$ $\begin{array}{llllll}0.3396936750868798 & 0.2200548598477409 & 0.0213672750706763\end{array}$ $\begin{array}{lllll}0.8396450477707514 & 0.2200169710104778 & 0.0214775967290624\end{array}$ $\begin{array}{lllll}0.3392577080249518 & 0.7194876603950898 & 0.1721995019133390\end{array}$ $\begin{array}{lllll}0.8392098746863191 & 0.7193502943258159 & 0.1721199507085143\end{array}$ $\begin{array}{lllll}0.3379981750622541 & 0.2190977689587036 & 0.3208756215750185\end{array}$ $\begin{array}{lllll}0.8391846699031861 & 0.2194925637239382 & 0.3210506345854023\end{array}$ $\begin{array}{lllll}0.3377771310477525 & 0.7152271596239538 & 0.4701121777534429\end{array}$ $\begin{array}{llllll}0.8364477219510271 & 0.7146100917153534 & 0.4736780167251168\end{array}$ $\begin{array}{llllll}0.3713809093873959 & 0.0567422192884034 & 0.0280293422355483\end{array}$ $\begin{array}{lllll}0.8716631838281046 & 0.0567390714548954 & 0.0280432800346617\end{array}$ $\begin{array}{lllll}0.3690177472880996 & 0.5564648811650417 & 0.1793102293870744\end{array}$ $\begin{array}{lllll}0.8690500850512396 & 0.5563846695411029 & 0.1792944684559906\end{array}$ $\begin{array}{lllll}0.3668038250288975 & 0.0560291579883844 & 0.3281113496269252\end{array}$ $\begin{array}{lllll}0.8682130788603830 & 0.0564768690414862 & 0.3283965635514881\end{array}$ $\begin{array}{lllll}0.3688442858183005 & 0.5517656133199460 & 0.4760519453739323\end{array}$ $\begin{array}{lllll}0.8700547653429087 & 0.5520808314322015 & 0.4799152657090133\end{array}$ $\begin{array}{lllll}0.2584117149864649 & 0.1070418775422777 & 0.9969169845086590\end{array}$ $\begin{array}{lllll}0.7589933546442543 & 0.1070295137349464 & 0.9968389025121766\end{array}$ $\begin{array}{lllll}0.2578169851086337 & 0.6064379545882436 & 0.1478570682143658\end{array}$ $\begin{array}{lllll}0.7577076911394116 & 0.6061387176064957 & 0.1478616143999786\end{array}$ $\begin{array}{llllll}0.2546295300100383 & 0.1062869115265760 & 0.2970187330410544\end{array}$ $\begin{array}{lllll}0.7557760343685588 & 0.1063619856759558 & 0.2973321136804958\end{array}$ $\begin{array}{llllll}0.2518684644532507 & 0.6033211368895134 & 0.4466287330565158\end{array}$ $\begin{array}{lllll}0.7599890436181248 & 0.6014493271653846 & 0.4480355205650781\end{array}$ $\begin{array}{lllll}0.5155414509275077 & 0.4254338310858193 & 0.5908687762442180\end{array}$ $\begin{array}{lllll}0.4161435703941626 & 0.5872110000098530 & 0.6006351641367225\end{array}$

\section{References}

${ }^{1}$ Dion, M.; Rydberg, H.; Schroder, E.; Langreth, D.C.; Lundqvist, B.I. Van der Waals density functional for general geometries. Phys. Rev. Lett. 2004, 92, 246401

${ }^{2}$ Klimeš, J.; Bowler, D. R.; Michaelides, A. Chemical accuracy for the van der Waals density functional. J. Phys.: Cond. Matt. 2009, 22, 022201-1-5.

${ }^{3}$ Klimeš, J.; Bowler, D. R.; Michaelides, A. Van der Waals density functionals applied to solids. Phys. Rev. B 2011, 83, 195131.

${ }^{4}$ Roman-Perez, G.; Soler, J. M. Efficient Implementation of a van der Waals Density Functional: Application to Double-Wall Carbon Nanotubes. Phys. Rev. Lett. 2009, 103, 096102. 
$\left.{ }^{5}\right]$ T. Thonhauser, V. R. Cooper, S. Li, A. Puzder, P. Hyldgaard, and D. C. Langreth, Van der Waals density functional: Self-consistent potential and the nature of the van der Waals bond, Phys. Rev. B 76, (2007) 125112.

${ }^{6}$ Kresse, G.; Futhmuller, J. Efficiency of ab-initio Total Energy Calculations for Metals and Semiconductors Using a Plane-wave Basis Set. Comput. Mater. Sci. 1996, 6, 15-50.

${ }^{7}$ Kresse, G.; Furthmuller, F. Efficient Iterative Schemes for ab initio Total-energy Calculations Using a Plane-wave Basis Set. Phys. Rev. B 1996, 54, 11169-11186.

${ }^{8}$ Kresse, G.; Hafner, J. Ab initio Molecular Dynamics for Liquid Metals. Phys. Rev. B 1993, 47, 558-561.

${ }^{9}$ Blöchl, P. E. Projector Augmented-wave Method. Phys. Rev. B, 1994, 50, 17953-17979.

${ }^{10}$ Henkelman, G.; Uberuaga, B. P.; Jónsson, H. A climbing image nudged elastic band method for finding saddle points and minimum energy paths. J. Chem. Phys. 2000, 113, 9901-9904.

${ }^{11}$ Cady, H. H; Larson, A. C. Pentaerythritol tetranitrate II: its Crystal Structure and Transformation to PETN I; an Algorithm for Refinement of Crystal Structures with Poor Data. Acta Cryst. 1975, B31, 1864-1869.

${ }^{12}$ Perdew, J. P.; Burke, K.; Ernzerhof, M. Generalized gradient approximation made simple. Phys. Rev. Lett. 1996, 77, 3865-3868.

${ }^{13}$ Tsyshevsky, R.; Sharia, O.; Kuklja, M. M. Energies of Electronic Transitions of PETN Molecules and Crystals. J. Phys. Chem. C, 2014, 118, 9324-9335.

${ }^{14}$ Tsyshevsky, R.V.; Sharia, O.; Kuklja, M.M. Optical Absorption Energies of Molecular Defects in Pentaerythritol Tetranitrate Crystals: Quantum Chemical Modeling J. Phys. Chem. C 2014, 118, 26530-26542.

${ }^{15}$ Mullen, P. A.; Orloff, M. K. Ultraviolet Absorption Spectrum of Pentaerythritol Tetranitrate. $J$. Phys. Chem. 1973, 77, 910-911.

${ }^{16} \mathrm{Yu}, \mathrm{Z}$; Bernstein, E. R. Decomposition of Pentaerythritol Tetranitrate [C(CH2ONO2)4] Following Electronic Excitation. J. Chem. Phys. 2011, 135, 154305-154305-10.

${ }^{17}$ Cooper, J. K.; Grant, C. D.; Zhang, J. Z. Experimental and TD-DFT Study of Optical Absorption of Six Explosive Molecules: RDX, HMX, PETN, TNT, TATP, and HMTD. J. Phys. Chem. A, 2013, 117, 6043-6051

${ }^{18}$ Meier, A. R.; Wagnibre, G. H. The long-wavelength MCD of some quinones and its interpretation by semi-empirical MO methods. Chem. Phys. 1987, 113, 287-307

${ }^{19}$ Dawson, C. R.; Nelson, J. M. The Influence of Catechol on the Stability of o-Benzoquinone in Aqueous Solutions. J. Am. Chem. Soc. 1938, 60, 245-249.

${ }^{20}$ Albarran, G.; Boggess, W.; Rassolov, V.; Schuler, R. H. Absorption Spectrum, Mass Spectrometric

Properties, and Electronic Structure of 1,2-Benzoquinone. J. Phys. Chem. A 2010, 114, 7470-7478 